- Igygygerges

ges cougros S \&

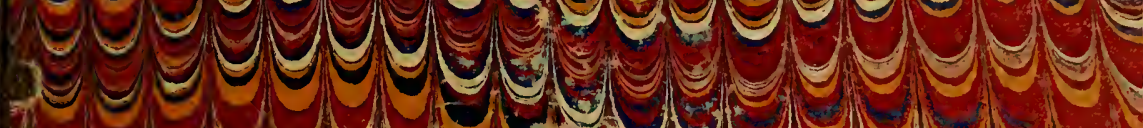
y y s s cy y y y y S Sy S s. yes 040

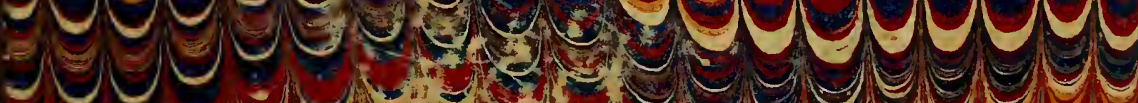
-

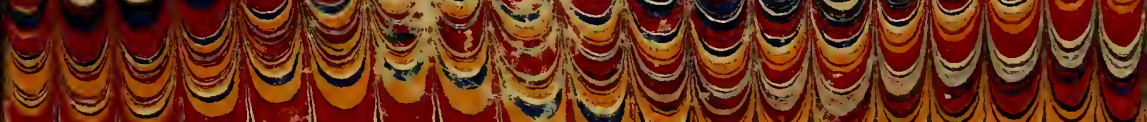
-

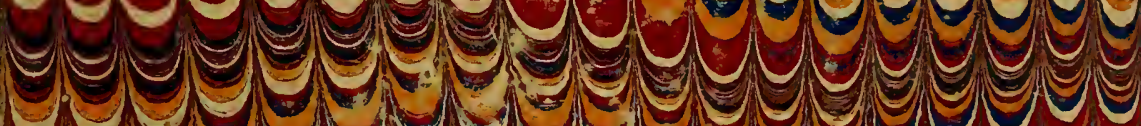
Y Y S

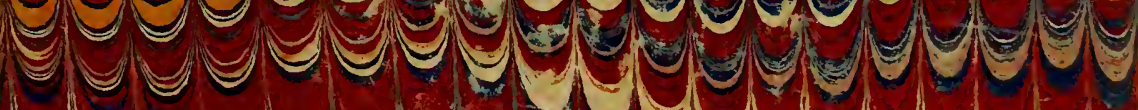
Maly gray y y e S S 象 S .

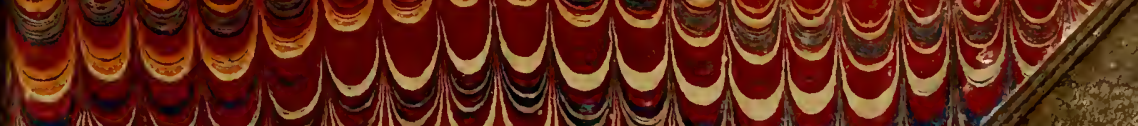

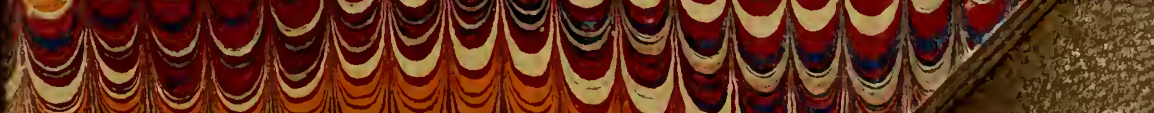
S 


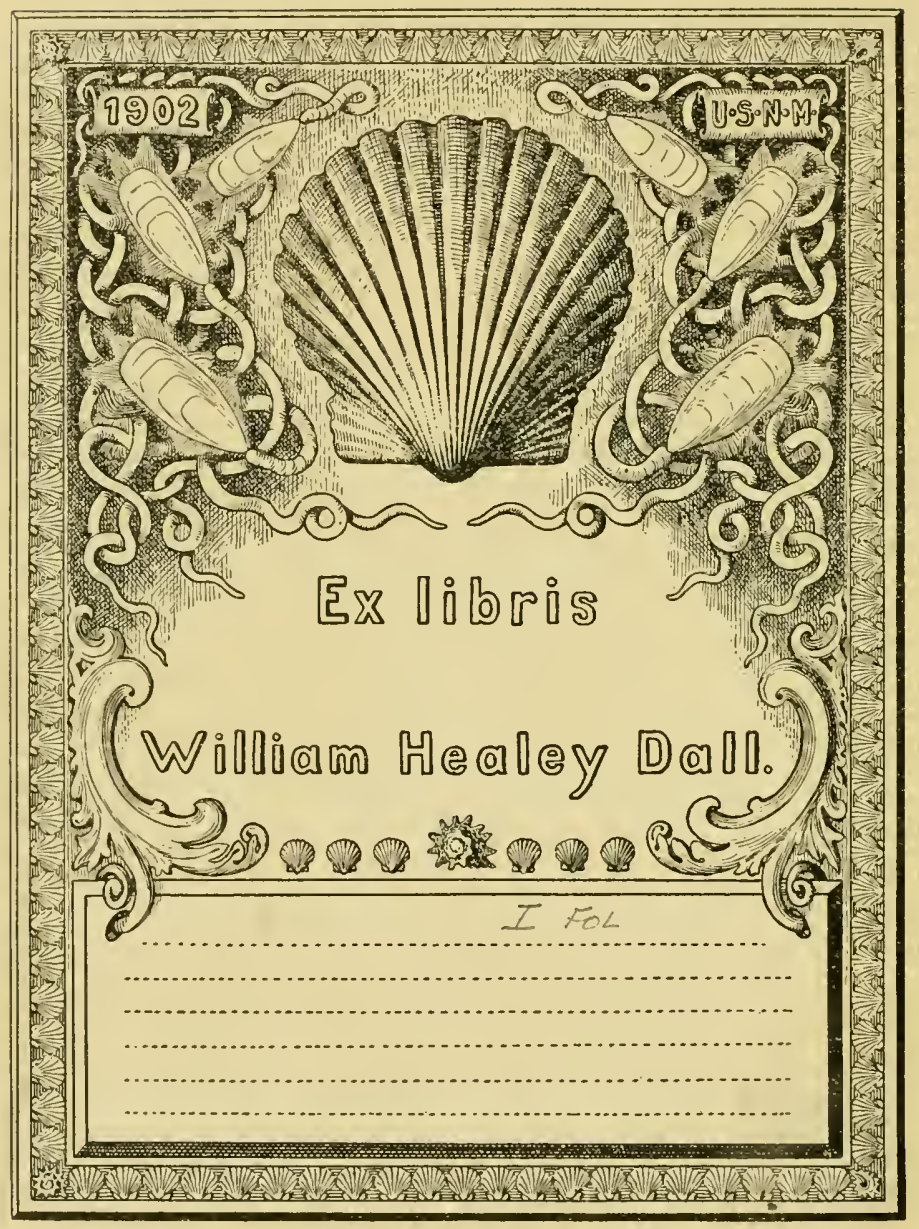


$I=-$

Division of Minlluake Sectional Library 




\section{A RCHIVES}

IIE

\section{Z00LOGIE EXPERIMENTALE}

\section{ET GÉNÉRALE}

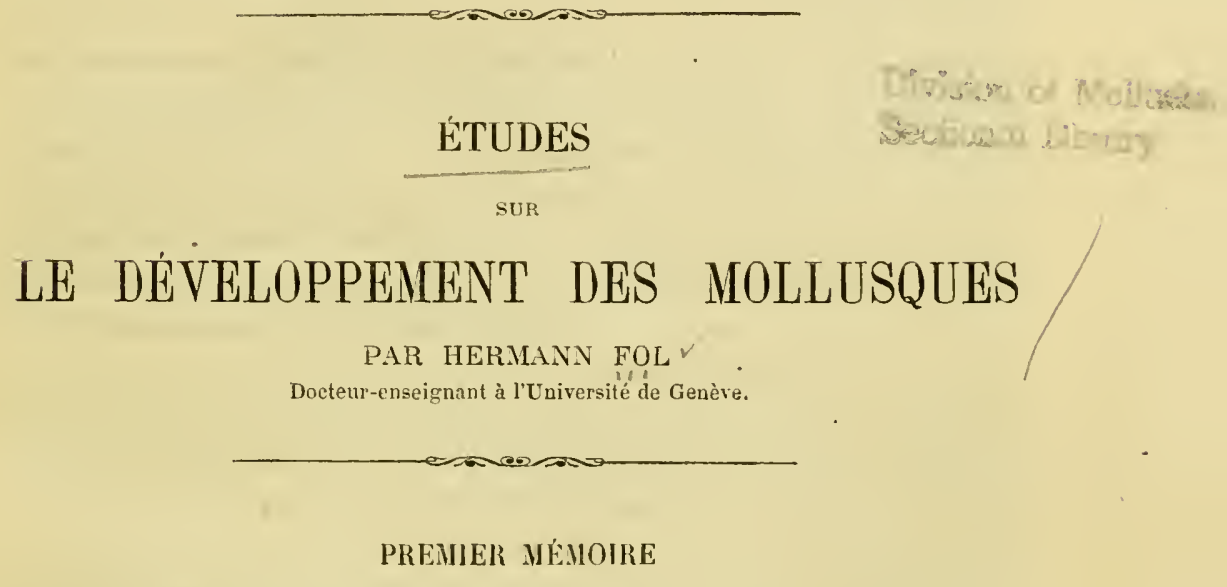

SUR LE DÉVELOPPEMENT DES PTEROPODES

INTRODUCTION.

Les études dont je me décide à livrer à la publicité un premier fragment, datent de fort loin. Déjà, du temps où j'étudiais auprès du professeur Gegenbaur les éléments de la science à laquelle je devais me livrer plus tard, la lecture des premiers travaux de mon maitre éveilla en moi un vif intérêt pour l'histoire naturelle des Mollusques, cet embranchemeni dont l'histoire peut être suivie d'une manière relativement si complète, à l'aide non-seulement de l'anatomie comparée et de l'embryogénie, mais encore à l'aide des abondants matériaux archéologiques que renferment les couches de la terre.

Ze but que je me proposais en abordant ee champ d'études était le suivant : arriver à la connaissance des traits fondamentaux de la for- 
mation embryogénique de ces animaux et de leurs principaux organes, en m’attichant surtout aux phénomènes de segmentation et de formation des feuillets blastodermiques; rechercher les procédés par lesquels les dirers organes se forment aux dépens de sphères de segmentation, ou des feuillets blastodermiques; voir si ces procédés sont comparables chez les diverses classes de Mollusques, s'ils peuvent ou ne peurent pas ètre ramenés an même type; mais ne conparer que les precessus londamentanx, en les distinguant antant que possible des phénomènes d'adaptation de la larve aux circonstances extérieures.

C'est dans le laboratoire de M. Gegenbaur, dans les années 1865 et 1866, que je commençai à m’occuper de l'anatumie et du déreloppement des Hollusques pulmonés : l'Arion, la Limace, la Succinée el l'Hélix furent les genres que j'étudiai. Plus tard, un voyage aux îles Gamaries me fournit l'occasion de commencer l'étude du développement des Mollusques marins; mais la pauvreté malacologique de cette côte m’empècha d'arriver an résultat que je désirais atteindre. Quelques notes sur l'évolution de la Phyllirhoë et de la Creseis furent le seul butin malacologique que je rapportai 'de celte expédition.

Messine, dont la richesse inépuisable a été à peine entamée par les nombreux travaux qui y ont été faits, devait me dédommager de ces déceptions.

Je profite de cette occasion pour témoigner publiquement ma gratitude à tous mes amis de Messine, pour l'intérêt qu'ils ont témoigné pour mes travaux et ma personne, et pour les nombreux services qu'ils m'ont rendus, afin de m'aider à atteindre le but que je me proposais. C'est en particulier à M. Victor Gonzenbach, consul suisse à Messine, que joffre mes remercînents les plus chaleureux.

C'est à Messine que j'ai pu commencer en 1870 l'étude de l'érolution des Céphalopodes; en 1871, je commençais celle des Ptŕropodes et des Hétéropodes, et au printemps de 1874 je terminais ces obserrations, du moins en ce qui concerne les Ptéropodes. Les observations que le lecteur a sous les yeux datent done des mois de mars, arril et mai des années 1871 et 1874. Mais les questions qui ont été résolues chez cette classe ont été déjà étudiées et en partie résolues pour beaucoup d'autres Mollusques. C'est la presque certitude que j'ai acquise. que beaucoup de faits énoncés pour les Pléropodes sont d'une vérité générale pour les Céphalophores, à quelques modifications près, et sont même dans une certaine mesure d'un intérêt général pour tous les 
Mollusques, qui leur domne une importance suffisante pour qu'ils méritent d'être immédiatement publiés.

Les difficultés que l'embranchement des Mollusques oppose aux efforts de l'embryogéniste, sont fort grandes, et expliquent eomment tant d'observateurs du plus grand mérite ont pu travailler sur ce sujet sans par'venir à l'épuiser. Les œufs des Céphalophores et des Lamellibranches sont pour la plupart holoblastiques et renferment une provision de nourriture, sous la forme de globules d'albumine et de graisse plus ou moins abondants et répandus dans tous les tissus embryonnaires. Les cellules embryonnaires sont en outre souvent peu définies et supportent mal l'action des réactifs. Les embryons sont pour la plupart trop petits et trop délicats pour pouroir être commodément dureis et étudiés à l'aide de coupes. Et enfin l'apparition hâtive de cils vibratiles sur leur surface, et la rotation qui en résulte, jointe au peu de transparence de la eoquille et des tissus de beaucoup de larves, achèvent de rendre très-dilficile la solution des questions les plus importantes d'embryogénie comparée.

Pour surmonter ces obstacles, j'ai commencé par chercher, parmi les Mollusques eéphalophores, quels étaient ceux dont les embryons étaient les plus faeiles à étudier', pour constater ensuite les rapports et les différences qu'ont avec ceux-ci les objets d'une étude plus diffieile.

Les œufs les plus favorables que j’aie rencontrés sont d'abord ceux des Ptéropodes; puis, parmi les Gastéropodes, ceux de la Phyllirhoë et de l'Actéon, dont je parlerai dans une autre occasion.

DE LA PONTE DES PTÉROPODES.

Les œufs des Ptéropodes sont très-faciles à obtenir. Il suffit de garder, au printemps, ces animaux dans des boeaux suffisamment grands pour obtenir dès le premier ou le second jour des chaines d'œufs, qui se développent à merveille. Il est vrai que les adultes ne donnent en général qu'une ponte; faute de trourer la nourriture qui leur convient, ils dépérissent et meurent au bout de trois ou quatre jours. Mais ils ne sont pas rares dans la Méditerranée, et le courant de Charybde, en entrant dans le port de Messine, en amène chaque jour à la surface un grand nombre.

Les Ptéropodes sont hermaphrodites, chacun sait cela. La plupart de ces animaux pondent leurs œufs à une certaine heure de l'après-midi ou de la soirée. Ainsi, par exemple, la Cavolinia tridentata pond. géné- 
ralement le jour même de sa capture, une longue chaine d'œufs. Son heure est celle du coucher du soleil. La chaîne se compose d'une enreloppe glaireuse très-molle et élastique, présentant une série d'élargissements aplatis en forme de poches. Ces élargissements mesurent environ $4, \mathfrak{5}$ millimètres de diamètre et sont reliés entre eux par un cordon de 2 millimètres de large, y compris l'enveloppe glaireuse (pl. 1, fig. 1 et 2). Celle-ci a une épaisseur de 7 dixièmes de millimètre environ. Ces dimensions ne sont qu'approximatives et varient d'un individu à l'autre. Elles ne se rapportent qu'à des chaînes qu'on abandonne à elles-mêmes dans une eau tout à fait immobile, car l'élasticité de la chaîne est telle, que le moindre courant d'eau suffit pour la déformer et l'étirer jusqu'au multiple de sa longueur naturelle. Elle est si élastique, qu'elle reprend sa forme après avoir été étirée à dix fois sa longueur.

L'espace intérieur, circonscrit par l'enveloppe, est occupé par une gelée peu consistante et parsemée d'œufs (pl. 1, fig. 2). Les œufs sont au nombre de cent vingt-cinq environ dans chaque élargissement, en comprenant dans ce nombre ceux que contient la portion de chaîne qui relie un élargissement avec le suivant. Ces chiffres encore sont soumis à de grandes variations. Chaque reuf se compose, de même que chez tous les Ptéropodes : $1^{\circ}$ d'une membrane élastique assez épaisse pour présenter un double contour; $2^{\circ}$ d'un albumen parfaitement limpide; $3^{\circ}$ d'un vitellus plus ou moins coloré. Chez cette espèce, le vitellus est coloré en rouge saumon (pl. l, fig. 3 et suiv.). Il a un diamètre de 22 centièmes de millimètre. Le nombre des oufs que pond un animal adulte et bien portant varie énormément. La longueur de la chaîne pondue au coucher du soleil varie de 1 à š centimètres, présentant de deux à dix élargissements et, par conséquent, de deux cent cinquante à douze cent cinquante œufs. Les chaînes moyennes sont les plus fréquentes et se composent de six élargissements, avec sept cent vingt-cinq œufs environ.

Ces chiffres répondent-ils bien à l'état normal de l'espèce ? Ne sontils pas affectés par l'état de réclusion dans lequel on tient le parent? La question est difficile à résoudre, d'autant plus que l'espèce dont il s'agit ne vit guère en bon état, dans des bocaux, pendant plus de deux à trois jours et ne pond qu'une fois pendant cet espace de temps. Ce n'est que très-exceptionnellement que j’ai vu un même individu pondre deux fois dans l'espace de trois jours. D'après cette obserration la ponte n'aurait lieu qu'une fois tous les deux ou trois jours. Un 
fait vient à l'appui de cette opinion : lorsqu'on prend à la fois un grand nombre d'exemplaires de cette espèce, le soir venu, il n’y en a guère que la moilié qui se mette à pondre. Si l'on sépare dans deux aquariums ceux qui ont pondu et ceux qui n'ont pas pondu et que l'on renouvelle soigneusement leur eau, l'on voit ees derniers déposer, pour la plupart, leurs oufs le second soir, tandis que les premiers ne pondent pas une seconde fois. Ces observations semblent indiquer que l'état de réclusion ne modifie pas sensiblement l'acte de la ponte, du moins pendant les deux premiers jours. Ce qui vient encore confirmer cette opinion, e'est que j'ai pris plus d'une fois en mer des chaînes d'œufs de cette espèce, qui ne différaient en rien des chaînes produites en captivité.

Ainsi donc, si l'on veut se rendre compte de la prestation de matières organiques que réclament de ces animaux les fonctions de la reproduction pendant vingt-quatre heures, il faut diviser par deux la quantité de matière organique que renferme une chaîne d'œufs et y ajouter encore la quantité de sperme nécessaire à les féconder, puisque ces êtres sont hermaphrodites et se fécondent mutuellement, comme j’ai souvent eu l'oceasion de l'observer.

Laissons de côté dans ce calcul les enveloppes glaireuses des chaînes et des oufs, qui, quoique volumineuses, sont trop gonflées d'eau pour renfermer beaucoup de matières organiques, et ne considérons que les œufs. Caleulant le poids de chaque œuf d'après son volume, 135 dix-millièmes de millimètre cube, qui est facile à mesurer, et admettant que sa densité n'est que peu supérieure à celle de l'eau de mer, nous arrivons à trouver que le poids de trois cents œufs, moyenne journalière, est de 47 dix-milligrammes. Le poids d'un animal adulte étant de š décigrammes, ce chiffre représente la centième partic environ du poids de l'animal. Si l'on songe que l'œuf est une quintessence de substance organique et si l'on ajoute encore la produetion du sperme, qui, quoirque peu considérable comne poids, n’en est pas moins fatigante pour 'organisme, le spermatozoaire étant, lui aussi, de la matière vivante par excellence, l'on verra que la reproduction demande à ces êtres une clépense considérable.

Une question presque impossible à résoudre est celle de la durée de la ponte pour chaque individu. Les Cavolinia tridentata montent à la surface de la mer et pondent régulièrenent depuis le mois de lérrier jusqu'au milieu de juin. Mais sont-ce les mèmes individus qui se reproduisent pendant toute la saison, ou bien ne sont-ce pas plutòt des indi- 
vidus venant de diverses situations et de diverses profondeurs, qui se succèdent à la surface de l'eau, pour y eflectuer leur ponte pendant un temps plus oi moins court? Celle dernière supposition paraît la plus probable, surtout si l'on considère que la Cavolinia tridentata doit être, d'après la loi de l'adaptation des coulcurs aux milieux ambiants, un animal vivant près du fond et parmi les algues. La couleur bruneverdâtre lle l'animal, ses longs appendices rubanés, d'un vert tendre et ressemblant à s’y méprendre à certaines plantes marines, la lour'deur de sa natation intermittente, tout fait supposer que nous avons affaire à des animaux passant la plus grande partie de leur vie près du fond de la mer, dans la zone des algues.

La Hyalocylis striata est très-commune vers la fin du printemps; elle pond de gros œufs (de 3 dixièmes de millimètre de diamètre moyen), rangés en chapelet dans un tube. Ce tube se casse pendant la ponte par petits morceaux renfermant de trois à six cufs. La ponte a lieu tous les jours vers la fin de la journée et se compose d'une vingtaine d'œufs, représentant la centième partie du poids du parent.

La Hyalcea complanata a été très-rare à Messine pendant le temps que j’y ai passé, aussi n’ai-je que peu d'observations sur cetle espèce. Ses chaînes d'oufs ressemblent beaucoup à cclles de l'espèce précédente; elles sont un peu plus petites et plus courtes, les ceufs de dimensions un peu infórieures et plus pâles de couleur. La ponte a lieu aussi vers le coucher du soleil.

La charmante petite Hyalce labiata est assez rare à Messine. Elle pond ses cufs, an nombre d'une trentaine, dans une gaîne en forme de gousse de pois, plus résistante que celle des espèces précédentes. Les parois de cette gaîne ont $\mathbf{2}$ dixièmes de millimètre d'épaisseur (pl. Tl, fig. 2). Les œufs ont un diamètre moyen de 23 centièmes de millimètre. Le vitellus, légèrement jaunàtre, mesure de 20 à 24 centièmes de millimètre. Je ne saurais dire à guels intervalles se renouvelle cette ponte, en sorte qu'il scrait inutile de chercher à calculer ce qu'elle représente de matière organique. Toujours est-il que ces aufs sont presque aussi gros que ceux de Cavolinia et, par conséquent, énormes en comparaison du poids du parent. Comme ils sont nombreux dans chaque ponte, celle-ci ne doit se faire qu’à des intervalles éloignés.

La Cleodor lanceolata est fort commune à Messine. Elle pond chaque jour dans le courant de l'après-midi, généralement vers les trois ou quatre heures, une petite chaîne d'œuls. Cette chaîne se compose d'un tube glaircux élastique dont les parois ont environ 
1 millimètre d'épaisseur et laissent un lumen cylindrique de $1^{\mathrm{mm}}, 2$ de diamètre. C'est dans ce lumen que se trourent les œufs, rangés en ligne (pl. VIl, fig. I et 2). Ils ont un diamètre moyen de 22 centièmes de millimètre, soit 49 millièmes de millimètre cube on volune. Le vitellus, de couleur jaunâtre, a un diamètre moyen de 19 centièmes de millimètre. En individu bien nourri pond en moyenne vingt œufs par jour, seulement il faut remarquer que le poids de l'animal, ne comportant que $S$ centigrammes, est extrêmement inférieur à celui de la Cavolimia, tandis que ses cufs sont presque aussi gros que ceux de cette dernière. La prestation de matièrc organique, pour les cufs seulement, pendant la durée de la ponte, est donc d'un quatre-ringtième du poids du parent. L'on rencontre pendant toute la durée du printemps des individus en roie de reproduction; mais il est à noter que, chez cette espèce, comme chez tous les Ptéropodes thécosomes en général que j’ai eu l’occasion d'observer, ce ne sont que les individus tout à fait adultes qui se reproduisent. Les individus jeunes, déjà tout près d’ąrriver au terme de leur croissance, sont encore complétement stériles.

La Styliola subulata abonde dans les eaux du détroit de Messine. Elle nage à la surface en quantités innombrables dans les belles journées du printemps. Malheureusement ses aufs sont d'une petitesse extrême, et, comme ils s'isolent peu après la ponte, il devient assez difficile de les utiliser pour l'étude. On les tronve dans les vases où l'on tient les animaux adultes dans les mois de mai et de juin, mais il m’a été impossilule de me rendre compte des proportions qui existent entre le poids de leur ponte et celui du parent.

La Creseis aciculata se rencontre fréquemment à la surface. Toutefois son apparition dans le port de Messine est très-capricieuse. Parfois on la troure par myriades. Dans d'autres moments, elle est beaucoup) moins fréquente, et il derient très-difficile de s'en procurer des exemplaires adultes. Pendant tout le printemps de 1874, je n'en ai pris que rleux exemplaires adultes, quoique les jeunes fussent assez abondants, et il ne m’a pas été possible d'en obtenir plus d'une ponte; quantité tout à fait insuffisante pour l'étudle, ru l'extrême pelitesse el la délicatesse des cuf: La ponte de Creseis aciculata a la forme d'une bandelette aplatie, presque aussi large que longue, et présentant à l'nil nu l'apparence d’un morceau de membrane transparente. Elle se compose d'une enveloppe glaireuse mince et d'une cinquantaine d'œufs disposés en une seule couche et très-rapprochés les uns des autres. 
L'acte de la ponte se fit, dans le seul cas que j'eus l'oceasion d'observer, dans le courant de l'après-midi.

La Limacine se montre parfois en grand nombre, mais je n'ai pas observé sa ponte, soit que la saison ne fùt pas venue, soit que les œufs aient échappé à mon observation.

Les Théensomes à coquille cartilagineuse et interne comptent trois représentants dans le détroit : Cymbulia Peronii et quadripunctata et Tiedemannia neapolitana.

De ces trois espèces, c'est la première senlement qui a servi à mes études. Les deux dernières ne sont pas assez fréquentes à Messine pour répondre aux besoins de l'embryogéniste.

La Cymbulia Peronii se rencontre souvent à Messine; elle est d'une fécondité exemplaire. Un individu adulte pond pendant plusieurs jours de suite, aussi longtemps qu’on peut le garder en bonne santé, de longues chaînes glaireuses cylindriques, el qui paraissent renfermer une série de chambres oblongues et fermées, accolćes bout à bout de facon à former un long chapelet (pl. VIII, fig. 11). Une chaîne moyenne se compose d'une dizaine de ces chambres ou chaînons, et chaque chaînon renferme environ quarante oufs. Un animal adulte produit jusqu’à trois et quatre chaînes par jour, ce qui donne une moyenne journalière de douze cents cul's. La ponte a lieu à toutes les heures de la journée. L'œuf a un diamètre moyen de 14 centièmes de millimètre et renferme un vitellus jaunâtre de $\mathbf{1 1}$ rentièmes de millimètre. Le poids total des wuis pondus dans l'espace de vingtquatre heures représente environ la soixantième partie du poids de l'animal. La saison de ce charmant Ptéropode commence en mars, veut-être avant, et se prolonge jusqu'en juin.

Si nous comparons le rapport du poids de Cymbulin au poids de sa ponte, avec ceux que nous arons déjà obtenus pour d'autres espèces, nous sommes frappés de voir qu’ils diffèrent beaucoup moins que le nombre des reufs selon les espèces. Enn d'autres termes, la quantité de matière organique que demande à l'organisme la fonction de reproduction, varie d'une espèce à l'autre beaucoup moins qu'on ne le croirait au premier abord.

Mais, tandis que chez telle espèce cetie malière est employée à former un petit nombre de germes bien ponrus de nourriture et qui donneront rapidement de grosses et puissantes larves bien nourries, chez telle autre espèce ectte mème quantité de matière est répartie entre un nombre d'mufs beancoup plus grand, en sorte que chaque 
germe sera moins gros, moins fort, moins bien pourvu de nourriture; mais, on même temps, le grand nombre de ces germes donnera plus de chances pour que l'un ou l'antre d'entre enx échappe à tous les dangers de la lutte pour l'existence et parvienne à l'état adulte.

Je ne songe nullement à contester la loi, bien connue, qui statue que le nombre de grermes va en diminuant à mesure que l'on remonte dans l'échelle animale, lantlis que le parent donne à chacun d'eux une provision de nourriture plus grande, les soigne et les protége plus longtemps. Cette loi est universellement reconnue ; mais chacun sait aussi qu’elle n’est vraie que dans ses traits généraux et qu’elle souffre de nombreuses exceptions, lorsqu'on cherche à la suivre dans les détails. Je ne fais ru'apporter un nouvel exemple à ajouter aux nombreux cas déjà connus, et qui prouvent que l'on ne doit pas chercher à appliquer la loi dans la comparaison entre les espèces d'un petit groupe. Tn effet, les divers Ptéropodes thécosomes sont tous à peu près sur le mème échelon de perfection organique. C'est à peine si l'on peut placer quelques genres au-dessus des autres. Or ces genres qui paraissent un peu plus perfectionnés, un peu plus éloignés du type primitif, sont précisément les genres à coquille interne ef cartilagineuse, qui se distinguent par l’abondance et la petitesse relative de leurs oufs. Si la loi se vérifiait jusque dans ses moindres détails, c'est le contraire qui derrait avoir lieu.

A mesure que les êtres se perfectionnent, qu'ils augmentent leurs moyens de défense, que la vie individuelle s'allonge, ils ont un avantage croissant à ne produire qu'un petit nombre de germes et à les protéger le plus longtemps possible, le germe ayant un plus long chemin, une évolution plus compliquée à parcourir pour arriver au point où le jeune animal peut se suffire à lui-mème. Mais cette règle, qui est vraie dans ses principaux traits, ne samrait tenir bon lorsqu'il s'agil de comparer des animaux aussi peu différents que le sont les divers genres des Plérołodes thécosomes. Ici les rapports si compliqués et si multiples des êtres entre eux et avec les milieux ambiants, rapports que nous ne pouvons encore que deviner, tant nous connaissons peu les véritables conditions d'existence des animaux marins, doivent amener des résultats impossibles à prévoir à prori.

J'ai cru utile de noter ces faits, en attendant qu'une connaissance moins superficielle des conditions biologiques des êtres vivant dans la mer permette de chercher leur explication. Je me borne à observer qu'à mes yeux ils n’infirment en rien la loi générale dont il a été 
question; ce sont lit de ces exceptions qui ne confirment ni n'infirment lit règle; ils sont trop spéciaux pour que la loi leur soit applicable.

Les Ptéropodes gymnosomes sont bien moins communs que les précédents dans le détroit, du moins ì l'élal adulte.

de n'ai pris pendant tout le printenps de 1874 que deux on trois exemplaires adultes de Cliopsis Krohnii, autant de Pneumodermon violaceum et eing ou six exemplaires de Clio aurantiaca; mais, quoique ces animaux vivent fort bien en eaptivité pendant des semaines de suite, je na ai pu obtenir des wufs que de cette dernière espèce.

Les Clio aurantiaca dont je gardais quelques exemplaires dans un bocal, n’ont commencé à pondre que huit ou tlix jours après leur capture. Ils m'ont fait dans l'espace de quinze jours, du 6 au 20 mai, cing pontes à toutes les heures du jour et de la nuil. La ponte, à peu près sphérique, d'un diamètre de $4^{\mathrm{mm}}, \breve{3}$, se compose d'unc enveloppe glaireuse et d'un contenu également glaireux, mais un peu noins dense que l'enveloppe (pl. X, fig. 1). C'est dans ce dernier que sont disséminés les culs, au nombre de soixante-dix à quatre-vingts et présentant un diamètre de 3 dixièmes de millimètre. Le vitellus, incolore, peı chargé de matière nutritive, mesure 14 centièmes de millimètre.

Ces données sur la ponte des Pléropodes gymnosomes sont, nn le voit, trop incomplètes pour me permettre de faire des généralisations sur les fonetions de reproduction de res animaux.

Les méthorles employées pour me procurer des wufs de Ptéropodes découlent de ce que je viens de dire de leur reproduction.

J'allais à la pêche au moment où le courant de Gharybde entre dans le port de Hessine, entrainant avec lui et amenant à lit surface, peut-être malgré eux, des myriades d'animaux pélagiques de toute nature. L'heure à laquelle ee courant commence à entrer dansle port varie d'un jour à l'autre comme lit marée. C'est dans les momenis où le courant entre de grand matin que mes pèches étaient les plus fructucuses.

La méthode de pêche, que j’ai déjà indiquée dans mon travail sur les Appendiculaires, consiste ì écrémer la surface de l'eau ì l'aide d'une coilfe de mousseline que l'on retire de temps en temps, mais seulement assez pour que le fond te la coiffe reste flothant dans la mer. Dans ce fond de coiffe se trouve dlonc une petite quantité d'eau qui renferme les animaux pris; ju puise cette eau au moyen d'un verre, que je remplis ainsi d'une réritable soupe d'ètres virants. A 
l'aide de tubes, je retire de là immédiatement les animaux qui servent à mes études, el les isole, chaque espèce à part, dans des boeaux pleins d'eaı de mer limpide, qui sont rangés dans le fond du bateau, dans des paniers à bouteilles.

Si l'on pratique cette pêche pélagique eomme je l'ai vu faire à tant de naturalistes; retirant la coiffe de l'eau entièrement et la retournant dans de grands bocaux que l'on remporte ensuite tels quels à la maison, l'on obtient une quantité d'ètres mutilés, nageant dans une eau trouble. Pendant le transport, les animaux blessés aehèvent de mourir; beaueoup d'entre eux, tels que les Aleiopes ou les Ciœlentérés, produisent des liquides vénéneux, des glaires, remplis de nématocystes, qui rendent malades eeux des animaux qui ont échappé par miraele à ces rudes manipulations, si bien que l'on ne trouve plus en arrivant qu'une masse informe de blessés et de cadavres qui, mêlés aux impuretés de l'eau et de la eoiffe, forment une couche épaisse au fond du bocal.

Les naturalistes dont je parle ramassent ce dépôt (Seemulder) à l'aide de tubes ou de pipettes, l'examinent dans des verres de montre et en retirent les corps des animaux qu'ils veulent étudier. Et telles sont les méthodes qu'on a employées pour faire une foule d'ouvrages qui font encore autorité dans la science! Est-il étonnant ajrès cela que l'on sache encore si peu de choses sur la vie, les mocurs, l'apparence, les fonctions normales des animaux pélagiques? Est-il étonnant que la plupart des planches qui les représentent, ne nous donnent que des carieatures à peine reconnaissables de ces merveilles de la nature?

J'insiste sur ce sujet, parce que j’ai eu l'occasion de m'assurer que, malgré les indications que j’ai données dans mon mémoire sur les Appendiculaires, l’on eontinue à employer ces méthodes surannées.

Pour les gros animanx pélagiques, tels que les gros Ptéropodes, la méthode est bien plus simple; il suffit de les puiser au moment où ils arrivent à la surface. Mais il faut toujours aroir soin de ne pas les mettre dans le même bocal arec d'autres animaux. Il faut les isoler par espèces et leur donner une quantité suffisante d'eau de mer bien limpide.

Les pontes des Ptéropodes sont, à peu d'exceptions près, trèsfaciles à voir. Pour l'élevage ce sont des verres à fond plat et à bords peu élerés qui m’ont rendu les meilleurs servires. Il convient seulement de les suivre, et de faire attention qu'il ne s'y déreloppe pas 
d'Infusoires. Sil'on remarque la présence de ces Protozoaires, il faut aussitôt changer l'eau, en transportant la chaîne successivement dans plusieurs récipients pleins d'ean de mer fraîche, et, si quelques oufs ou quelque portion de ehaîne se trowrent déjà gâtés, il faut enlever immédiatement toute la portion affectée.

Au moment où les larves sont près d'éclore, il est bon de déchirer la chaine afin de leur permettre d'éclore plus aisément. En pleine mer les courants doivent disjoindre et déchirer l'enveloppe; il faut imiter artifieiellement cet agent, qui manque aux œufs élevés en captivité. Si la larve ne réussit pas à sortir de la chaîne au moment opportun, elle sonffre et meurt; les carlavres infestent l'eau, les infusoires s’y développent et les larres encore saines périssent bien vite.

Lorsque les larves sont écloses, il est conrenable de les transporter dans un grand bocal de forme ordinaire, plein d'eau bien pure.

Une remarque que j’ai faite pourra être utile aux personnes qui s'occuperont par la suite de l'embryogénie des Céphalophores. Les cuf́s tenus all frais se développent lentement et sont beaucoup plus facilement affectés par les conditions anormales où ils se trouvent et qu'ils subissent pendant plus longtemps. Pour suivre le développement complet d'une espèce, l'été est la meilleure saison ; en hiver l’on peut élever artificiellement la température de l'eau, en plaçant les bocaux qui contiennent les oufs, près d'un fourneau. Les larves prennent alors leur développement en peu de jours au lieu d'y mettre un mois et davantage, el l'on réussit souvent à les garder jusqu'à la perte des organes larvaires et à la formation du jeune animal. Ce sont donc les espèces à développement rapide qui valent le mieux, si l'on veut suivre la métamorphose de la larve. Les seuls auteurs qui aient jusqu'à présent réussi à observer cette métamorphose sur des larves élevées direetement des ceufs sont Max Schultze et M. de Lacazc-Duthiers; leurs observations ont été faites au milieu de l'été. La métamorphose du Tergipes a licu dans cette saison, une semaine environ après la ponte. Lc déreloppement des Hyaléacées à Messine au mois de juin est tout aussi rapide.

L'observation microscopique des aul's el des larves ne présente guère de difficultés, si l’on est mmi de compresseurs à lames parallèles d'un bon modèle. Ceux dont je me sers, ct qui m’ont été indiqués par Claparède, répondent à toutes les exigences de l'observateur.

Ces compresseurs sont surtout précieux pour des recherches qui doivent, comme celles-ci, êlre faites à de forts grossissements J'ai 
travaillé presque constamment avec une excellente lentille à immersion $n^{\circ} 9$ de Hartnack. Les lentilles plus fortes n'ont pu être employées que rarement, à cause de la petilesse de leur distance focale.

Les oufs et les larves des Ptéropodes sont si transparents et si nets, qu'il est inutile de chercher à les durcir et à en faire descoupes. L'on peut tout roir en observant seulement l'animal frais. Les acides acétique, osmique, etc., peurent, dans certains cas, être employés pour rendre plus visibles des organes qui, par leur extrême transparenc n'attirente, pas suffisamment le regard. T'outefois, la position de l'organe une fois reconnue, c'est toujours l'étude de l'embryon vivant qui déroile le mieux les secrets de son organisation.

DIVISION DU MÉMOHLE.

Avant de passer à l'exposition des observations que j'ai faites, des résultats que j’ai obtenus et des vues nouvelles qui en découlent, il sera bon de rappeler les nombreuses études faites sur des sujets analogues par d'autres observateurs. Ne parler que des travaux relatifs à l'embryogénic des Ptéropodes, serait un procédé très-injuste, puisque beaucoup de découvertes faites sur d'autres Mollusques sont applicables à cet ordre.

Bien loin de chercher à déprécier les travaux de mes devanciers pour augmenter l'effet de mes propres observations, je m'atlacherai à montrer que presque toutes les idées nouvelles que j’émettrai ont déjà été entrevues, que les faits qui leur servent de base ont été déjà observés, tantôl chez l'un, tantôt chez l'autre des types de Mollusques gastéropodes clont le développement a été étudié. Je trouverai dans cette concordance une confirmation de mes opinions, en même temps qu'une preuve de la généralité des faits observés chez un petit nombre de types. J'aurai enfin l'occasion de rendre justice au talent ei à la persévérance que d'autres savants ont déployés, aux patients labeurs qui nous ont guiclés jusquau point où nous sommes et nous ont permis d'aller un peu plus loin qu'eux, pour être bientôt devancés à notre tour.

Après cet exposé historique, que je chercherai à rendre aussi complet que le permetient les matériaux bibliographiques dont je dispose, et qui fera l'objet de mon premier chapitre, j'entamerai l'exposé de mes propres études. Je conmencerai par les premiers phénomènes intimes qui président au processus de la segmentation. Ju parlerai 
des règles de la formation des premières cellules, de leur arrangement, en un mol de la morphologie des stades de la segmentation.

Je ferai voir ensuite comment ces amas de cellules, d'une régularité frappante, se modifient et croissent 'pour' donner naissance aux feuillets blastorlermiques. Procédant encore plus avant, je montrerai par quels changements ces feuillets donnent naissance aux divers systèmes d'organes de la larve. Ce sera mon second chapitre comprenant la priziode embryonnaire.

Te montrerai ensuite comment ces organes ébauchés se différencient, se perfectionnent, se forment, en un mot, phénomène que je comprendrai sous le nom de période larvaire, et qui fera l'objet de mon troisième chapitre.

Les transformations que subit la larve pour devenir semblable au parent, la métamorphose et la croissance des direrses parties seront réunies dans le quatrième chapitre.

Je donnerai une attention spéciale à ceux des organes qui prennent leur plein développement chez la larve pour rediminuer ensuite, aux organes larvaires ou transitoires.

Je parlerai ensuite des différences que présente le déreloppement des divers types de Ptéropodes; j'exploiterai au point de rue de la classification les faits embryogéniques anciens et nouveaux que j’ai pu réunir, tant pour arriver à une subdivision rationelle du groupe que pour établir les affinités de cette famille avec les autres Mollusques. J'étudierai les traits fondamentaux communs à l'évolution de tous ces animaux. Ce sera mon cinquième et dernier chapitre.

Tel est le partage de ce mémoire. 


\section{HISTOIRE ET BIBLIOGRAPHIE.}

Il est peu d'embranchements, dans le règne animal, qui aient été farorisés d'un nombre aussi grand de travaux embryogéniques que celui des Mollusques. En jetant un coup d'œil sur l'index bibliographique qui accompagne ce mémoire, l'on y trouvera les noms de presque tous les hommes marquants en zoologie.

Passer en revue tous ces travaux successivement, en donnant de chacun un extrait, nécessairement très-incomplet, serait faire un travail ingrat el peu utile. Je préfère procéder par ordre de matières. Je prendrai done successivement les diverses périodes de développement et les différents organes, examinant toutes les données qui s'y rapportent. 11 est d'autant plus nécessaire de faire une revue consciencieuse des résulats acquis, que Keferstein, qui a trop tôt succédé à Bronn dans la continuation du Thierreich, a donné des travaux relatifs aux Céphalophores et aux Céphalopodes un résumé plein d'erreurs.

Je me vois obligé, à mon grand regret, de laisser de côté, pour le moment, dans cette esquisse, les travaux relatifs à l'embryogénie des Brachiopodes d'une part et des Céphalopodes de l'autre. L'état actnel de nos connaissances sur les Brachiopodes ne permet pas de comparer arec fruit leur développement avec celui des autres Mollusques. Quant aux Céphalopodes, un travail considérable va être publié par Ussow, et il convient d'attendre l'apparition de cet ouvrage avant de résumer les résultats obtenus sur ce groupe important. Je me bornerai done à deux classes : celle des Céphalophores et celle des Lamellibranches, et au groupe intermédiaire des Solénoconques.

Les faits rapportés par les anteurs anciens et ceux du moyen àge n'ont guère qu'un intérèt historique. Les observations embryogéniques y sont extrêmement rares et trop superficiclles pour pouvoir ètre encore utilisées. Les travaux d'Aristote (voyez Index bibliographique, $n^{0}$ I) renferment (quelques notices assez justes de ce genre. Les questions physiologiques de la génération sont traitées plus au long dans les ourrages ancicns; mais les discussions interminables que l'on rencontre sur la question des générations spontanées et sur la génération sexuelle n’ont que pou d'intérêt pour nous. les travaux; jusqu'au milieu du siècle acluel, traitant de ces sujets, ont été recueillis el résumés jar' 
de Blainville (Index, $x \mathbf{I x}$ ), par Carus (xxi), par Humbert (cxxxv) et par de Lacaze-Duthiers (xcıv). Les diverses formes d'enveloppes des œufs ont eu anssi le don dattirer heaucoup l'attention des auteurs du siècle dernier el du commencement de ce siècle, jusqu’à Lund (xxı), qui donne un résumé très-complet des observations recueillies jusqu'alor's, et qui eroit pouvoir baser la classilication des Mollusques sur la forme de leur ponte; point de vue insoutenable, ainsi qu’on ne tarda pas à le reconnaître (xLvn).

Les travaux antérieur's à Schwann et à von Baër ne pouvaient guère traiter que de la forme extérieure de l'embryon, et cela à un point de vue plutôt physiologique et téléologique. Comment, en effet, eût-il été possible de concevoir les véritables procédés de développement avant la publication de la théorie cellulaire? ayant que les travaux de von Baër eussent fondé la véritable morphologie embryonnaire, celle des feuillets, eussent élargi et rectifié la notion des types telle que l'arait émise Cuvier? Aussi royons-nons les travailleurs de cette époque se perdre dans la recherche de rapports impossibles à établir, en prenant pour base le petit nombre de faits superficiellement observés que l'on connaissait. C'est en rain que des naturalistes aussi conseiencieux que Carus ( $x \mathrm{xi})$, que Dumortier ( $\mathrm{x} \times \mathrm{x} 111)$ el autres, donnèrent des descriptions aussi exactes et aussi minutieuses que le permetlaient les moyens optiques imparfaits qu'ils possédaient el les connaissances insuffisantes de l'époque. La science n'en profita guère et les spéculations de la philosophie naturelle y trouvèrent seules leur aliment. Pour caractériser cetle tendance et ses exagérations, qui se produisirent encore pendant plusieurs années après les découvertes de Schwann et de von Baër, je citerai textuellement quelques-umes des conclusions qui terminent les travanx de Dumortier (xxxus) et de de Serres (xxxy). D'après Dumortier' :

"L'embryon culbute sans cesse sur lui-même par un mouvement automatique, l'extrémité postérieure en avant. En tournant ainsi sur lui-mème, les matières nerveuses qui se forment sont nécessairement cmportées à l'extrémité postérieure du tourbillon; là, elles s'agglomèrent, s'organisent el lorment bientôt le cervean... Ainsi, il n’existe pas chez l'animal de vie végélative, comme Bichat l'a dit; la vie végétative, e'est le développement centrifuge... L'observation si simple de la situation supère ou infère de la cicatrice nous explique le pourquoi de la différence d'organisation des animaux el des végétaux. Si le régétal se dirige vers le ciel, c'est que la cicatrice de son embryon 
s'est opérée au pôle zénith du globule; si l'animal rampe sur la terre, e'est que la cicatrice de son embryon s’est opérée à son pôle nadir. "

Et M. de Serres : "Les Mollusques sont des embryons permanents des Vertébrés et de l'homme... La coquille des Mollusques serait donc une caduque permanente, comme leurs branchies sont une allantoïde permanente, leur manteau un chorion permanent, leur canal intestinal un vitellus permanent... Leur déreloppement est un corollaire de la loi centripète des développements organiques. "

Mais avec les recherches de Laurent (xxviı), de Sars (xxxiv), de Lovén $(x \mathrm{Ll})$, l'on voit poindre une ère nouvelle; les spéculations sont laissées de côté, les observations s'accumulent et forment petit à petit l'ensemble imposant dont nous allons aborder l'analyse.

FORMATION DE L'OVULE DANS LES GLANDES GÉNITALES.

Le mode de formation de l'orule et la structure intime des glandes génitales sont une question assez controversée. Que la glande soit, du reste, seulement orarienne, ou bien ovarienne et testiculaire à la fois, les processus de la formation de l'ovule restent les mêmes.

Le stroma qui garnit intérieurement la membrane anhiste du follicule, est-il formé de cellules distinctes constituant un épithélium, ou bien est-ce un protoplasme continu et parsemé de nucléi?

Carus (xx1) admet cette seconde alternative en ce (qui concerne les Naïades. Ce protoplasme s'agglomère ensuite autour des divers nucléi et chaque agglomération s'entoure d'un thorion. Entre le chorion et l'ovule apparaît une couche d'albumine, et dans le nucléus ou vésicule germinative (que l'auteur nomme cicatricule) apparaît une tache germinative. Quelques années après, le mème auteur (xxvı) a donné une deseription à peu'près identique de la formation de l'ovule dans les genres Helix et Limax.

H. Meekel (uI) roit dans l'ovaire de Helix pomatia, avant la maturité, seulement des cellules, de vraies cellules nucléées. Celles-ci grossissent et s'entourent d'une membrane, la membrane vitelline. Le vitellus n'est autre que le protoplasme de la cellule primitive; la vésicule germinative, son nucléus. Pendant que le nucléus croît, il se différencie dans son intérieur plusieurs corpuscules albumineux, qui redisparaissent, à l'exception d'un seul : le nuclénle ou tache germinative.

ARCH, DE ZOOL. EXP. ET GÉN, - T. IV. 1875. 
Vou Nordmann (LuI) décrit la formation de l'ovule chez Tergipes Edwardsii, d'une manière qui n'a été confirmée par aucun autre observateur. Dans le stroma continu de l'ovaire, apparaitrait d'abord la tache germinative, que riendraient ensuite entourer la vésicule germinative, puis une conche de protoplasme à granules fins et une membrane. Aulour de cette membrane s'acemmulerail le vitellus à gros granules. La membrane séparant les deux espèces de vitellus disparaittrait, les vitellus se mêleraient et une membrane vitelline viendrait enfin envelopper le tout, complétant ainsi l’orule mùr.

D’après de Quatrefages (LxxiI), la vésicule de Purkinje apparaît la premic̀re dans l'ovaire de Teredo fatatis. Puis la tache germinative naît dans son intérieur. Une masse granuleuse vient entourer la vésicule germinative, el cette masse s'entoure elle-même d'une membran e mince.

F. Leydig (Lxxiv) confirme les données de Meckel en ce qui concerne Paludina vivipara. Le nucléole se compose de deux corpuscules accolés, ou d'un seul en forme de 8 de chiffre.

De Lacaze-Duthiers (xciv), d'après des observations portant sur un grand nombre de types de Lamellibranches (Unio, Cardium, Trigonella, Corlula, Arca, Spondylus, Pinna, ete.), admet que l'oraire se compose d'abord de véritables cellules, mais fait naitre l'ovule dans l'intérieur de ces cellules, qu'il nomme cellules mères. La vésicule germinative n'est que le nucléus de la cellule mère agrandi. L'ovule s'entoure d'un chorion d'abord à son extrémité libre, et en dernier lieu à l'extrémité étirée par laquelle il est attaché à la membrane du follicule. De là, la forme de bouteille que prend le chorion, houteille dont le col n'est autre chose que le micropyle de l'œuf.

H. Müller et G. Gegenbaur (xcv!) pour Phyllirhoe et Gegenbaur pour Actcen rapportent que chaque ovule n'est qu'une cellule de l'épithèle simple qui tapisse l'ovaire avant la maturité, cellule qui prend un grand développement.

Chez IJentalium, d'après de Lacaze-Duthiers (Cxi1), l'ovule se forme aux dépens des cellules qui constituent le stroma de l'ovaire, chaque ovule descendant directement de la majeure partie, sinon de la totalité de la cellule mère.

D'après Claparède (cxin) le follicule de la glande sexuelle chez $N e-$ ritina fluviatilis est tapissé d'un épithélium simple; la vésicule germinative de l'ovule proviendrait d'une cellule épithéliale tout entière, qui s'entourerait de substance vitelline. Cette vésicule renferme une 
tache germinative; la présence d'une membrane vitelline n’a pu être reconnue arec certitude.

C. Semper (cxy) décrit la glande sexuelle de Lymenceus, Planorbis, Succinca pendant le mois de décembre, comme une grappe de follieules, composés de l'enveloppe et d'un épithélium vibratile simple. Les cellules épithéliales sont très-transparentes el leur nucléus est si peu apparent, cu’il faut employer des réactifs pour le rendre visible. L'auteur pense, sans l'aroir ohservé directement, que ces cellules épithéliales se partagent par le travers, en une couche externe et une couche interne de cellules. Les orules dérivent directement des cellules de la couche externe, la membrane vitelline répondant à la membrane de la cellule épithéliale, le vitellus à son protoplasme, la vésicule germinative à son nucléus.

Von Hessling (cxxul) donne du développement del'ovule de Unio margaritifera une description qui ne s'accorde guère arec les opinions des autres auteur's. D'après ce savant, l'ovaire renfermerait une masse granulée et des cellules nucléées. Les nucléi de ces cellules se multiplieraient, se débarrasseraient de leurs cellules et resteraient ensuite nayés dans la masse granuleuse. Puis chacun de ces nucléi formerait, arec une portion de la masse gramuleuse, un amas qui s'entourerait d'une membrane, et piouluirait ainsi l'ovuie, le nucléus devenant résieule germinatire, la masse granuleuse devenant vitellus. Ce derniex, enfin, sécréterait la couche d’albumine, qui le sépare de la membrane vitelline.

Eisig (cxxx1), qui a suivi le déreloppement des organes génitaux chez Lymmeus, nous apprend que la glande génitale est tapissée, dans le jeune àge, d’un épithélium simple, et pense que les collules épithéliales se transforment directement eu produits sexuels.

Il résulte de ces données bien éparses que l'ovule des Céphalophores descend directement dians la plupart des cas, sinon dans fous, des cellules épithéliales du follicule glandulaire. Pour les Lamellibranches son mode de formation est encore bien douteux.

DESCENTE HE L'OVULE, FÉCONDATION.

Chez les Céphalophores, l'ovule mùr se compose d'un nucléus avec nucléole, d'un vilellus plus on moins granuleux et rl'une membrane vitelline. Tous les auteurs qui en parlent sont d'accord sur ce point: Carus (XI) pour Lymoeus, Neckel (LII) pour Helix et un grand nomber: 
de Gymmobranches, von Nordmamn (LI1) pour Tergipes, Leydig (cxxiv) pour P'aludina, Gegenbaur (sxrin) pour Limax, etc.

Pendant sa descente dans l'ovidncle et l'utérus, il est fécondé, puis entonté d'unc enveloppe albumineuse, d’une menbrane, et d'une enveloppe externe, qui rarie beaucoup de forme et de consistance, nonseulement d'une famille à l'autre, mais même chez des espèces roisines. Carus (xi) et von Nordmann (Li1) sont les seuls auteurs qui fassent dériver la membrane de l'œuf des Céphalophores de la membrane vitelline, el l'albumine de l'wuf, d'une excrétion de la part du vitellus. Les auteurs subséquents considerent, avec raison, la membrane et l'albumen de l'œuf des Céphalophores comme les produits de la sécrétion des organes sexuels efférents. Leydig (Lxxiv) a été l'un des premiers ì montrer clairement ce fait.

Le vitellus fécondé, et entouré de l’albumen, a-t-il encore la mêne structure que l'ovule primitif, ou bien a-t-il perdu sa membrane vitelline el son nucléus? Un grand nombre d'auteurs se prononcent pour celte seconde alternative, à savoir : Jacquemin (xL) pour Planorbis, puis van Beneden et Windischmann pour Limax (xLIV), et beaucoup d'auleurs subséquents, parmi lesquels je citerai Leydig (Lxiv) el Leuckart (xci1), qui ont clairement montré que, chez Paludina et Firoloïles, l'ovule, en descendant dans l'utérus, perd sa vésicule germinative et sát membrane vitelline. Presque fous les observateurs constatent l'ahsence d'unc nembrane vitelline autour du vitellus de l'ceuf fraîchement pondu. J.-L.-H. Laurent (xxvı) fait, il est rai, exception, et décrit le vitellus pondu de Limax comme pourvu d'une membrant propre. Mais rette obserration, qui date d'un temps où l'on ne faisait pas nıême la distinction entre un vitellus avant et après la segmentation, ne mérite pas grande attention. Les autres auteurs reconnaissent tous l'absence de celte membrane, à l'exception de Sars (xuil) pour Tritonia, Scyllece, Acolidia, Doris et Aplysia, de Fr. Müiller (cxix) pour Puntolimax, dont les observations sur ce point ne tardèrent pas ì ètre contredites par H. Rathke (Lxx), et de Koren et Danielssen (Lxxx et crx) pour Buccinum et Purpura, dont le témoignage, en ce qui concerne Purpura, est nettoment contredit par Selenka (cxxxi). L'opinion de Warneck mérite d’ètre notée, car elle atténue ces contradictions ( $x x v$ ) ; d’après ce naluraliste soigneux, le vitellus de Lymncus et de Limax est, après la fécondation, dépourru de membrane vitelline; mais la couche la plus exturne du ritellus est composée de protoplasme condensí, qui jome à peu près le ròle d'une membrane. mais 
diffère qle relle-ci en ce qüil u’est séparé par aucune limite du reste du protoplasme vitellin. J'ai moi-mème constaté l'exactitude de cette observation chez plusieurs Géphalophores marins.

Sur la présence ou l'ahsence d'une vésicule germinative dans l'ovule des Céphalophores au moment de la ponte, les opinions sont beaucoup plus partagées; et cela devait ètre, puisppue la résicule disparaît, reparail et disparait encore une seconde fois avant que la première segmentation ait lieu, ainsi que je le fais roir dans le troisième chapitre de ce mémoire. Tel observateur a ru les cufs à un certain moment, tel autre observateur a commencé à les suive un peu plus tòt ou un peu plus tard, et ils se contredisent avec une égale assurance, tous ayant raison jusqu'ì un certain point. Il serait inutile de citer une à une toutes ces observations peu instructives. Je me bornerai à noter celles qui ont été assez suivies pour jeter quelque lumière sur la généralité de ces phénomènes.

Jacquemin (xL), Leydig (Lxirv), Leuckart (xcu) ont ru les orules de Planorbis, Paludina el Firoloides perrlre leur vésicule au moment où ils arrivent dans l'utérus, c'est-à-dire vers l'époque de leur fécondation.

Cette vésicule fait-elle une réapparition passagère entre le moment de la fécondation et le moment uù les corpuscules excrétés sortent du vitellus? L'observation de Sars (xum) sur Doris et Étidia. d'après laquelle la vésicule germinative existerait le premier jour après la ponte, mais aurait disparu le second jour, n'est pas assez précise pour nous renseigner sur ce point. Von Nordmann (LiII) rapporte que, chez Tergipes, la vésicule et la tache germinative disparaissent peu d'heures après la ponte, après quoi les corpuscules excrétés opèrent leur sortie. Cette observation, malheureusement isolée, semble répondre à la question que j’ai posée plus haut, et indiquer que celte vésicule fait, au moins dans certains cas, une réapparilion pendant ce laps de temps.

Les observations de C. Vogt (cxul et de Gegenlbaur (ci) sur Acteon et’sur les Hétéropodes, d’après lesquelles la vévicule germinative persisterait et donnerait directement naisinec par division aux nucléi des sphères de segmentation, sont entachres l'orreur, ainsi que je m’en suis assuré par noi-mème. Le nucléus, en tant qu’élément morphologique, disparaît chez ces animanx lors de la sortie des corpuscules excrétés el de chaque segumentation. Ce qui persiste, re n'esi pas le unclérs, r'est une tache claise uniquement romposéc de proto- 
plasme, et qui contraste vivement, par sa transparence, aree la substance nutritive foncée qui l'en toure. Le nucléus apparaît et disparaît alternatirement dans cette tache claire; je décrirai plus loin, en détail, ces phénomènes pour les Ptéropodes gymnosomes, où ils se présentent à peu près les mèmes que chez les Hétéroporles et les Gymuobranches.

Warneck (LxXY) a très-bien compris et déerit le rôle que joue la vésicule germinative. Il ne parle, arant la sortie des corpuscules, que t'me tache elaire occupant le centre du vitellus, et ne possédant pas de membrane ni mème de contours nets. Cette tache se divise en deux moitiés, dont l'une reste au centre du vitellus, landis que l'autre arrive à la surface, et sor't sous forme de globules: les corpuscules excrétés (ou rle direction). Si cet observateur habile arait indiqué plus neltement la disparition du nucléus et s'il avait ru les étoiles moléculaires qui le remplacent, il ue m’aurait rien laissé à ajouter sur ce sujet. Les granules du ritellus avant la fécondation ne se modifient pas lorsqu'on les met en contact arec l'eau en écrasant l'ceuf. Après la fécondation, ces mênes granules, dans les mèmes circonstances, se gonflent el se changent en résicules, d’où l'auteur conclut que la fécondation a fait subir au vitellus une modificalion chimique profonde.

Les auteurs récents n’apportent ancune domnée nouvelle et restent même bien en arrière du travail dont 'je viens d’analyser une partie. Ainsi Keferstein et Ehlers (cxivn), dins un petit mémoire sur le développement d'Eolis, qui eùt eu quelque intérêt s’il eùt été fait ringt ou trente ans plus tôt, supposent, sans s'ètre domné la peine de le constater, que le nucléus de l'orulc persiste el se divise plus tard. LerebonIlet (cxirin) rapporte que le vitellus de Lymmous fraîchement pondu contient dans son centre deux résicules qui se dissolrent bieniôt. Quelques heures plus tard, se formeraient quatre vésicules, qui redisparaîtraient à leur tour; après yuoi aurait lieu la sortie des corpuscules de direction. Cette description, ontre qu'elle manque de clarté, a le lort de venir après lo travail fle Warueck, dont notre auteur ne paraît pas avoir eu commassince, el de ne cadrer aree aucun des faits ohservés chez le Lymnée ou chez d'autres animaux.

Chéz les Lamcllibranches et les Solénoconqunes, la membrane, qui entourait l'ovule dans l'ovaire, aequiert une indépendanec plus grande. Elle est souvent séparée du vitellus par une couche d'albumine, raisemblablement sécrétée par re dernier. possède un mieropyle et 
persiste pendant toute la première partie du développement. Ces faits étaient déjà comnus de J.-L. Prérost (.mi) et de Carus (xil).

Lovén(LXxr) rapporte que le vitellus pondu de Modiolaria mamornta n'a qu'une enveloppe membraneuse mince el accoléc à sal surface. Chez. Cardium parvum le vitellus nage dans un alhumen, qui est lui-mème renfermé dans une enreloppe à plusieurs conches. Chez Teredo, d'après de Quatrefages (cxxır ), le vitellus pondu est entouré de la même membrane qui l'entourait déjà dans l'oraire. membrane mince, transparenfe, et qui ne se détache de la surfice du vitellus qu’au moment où le fractionnement commence. De Lacaze-Duthiers (xciv) établit que le vitellus pondu est entouré d'une capsule épaisse chez les genres Ĺnio, Cardium, Trigonella, Corbula et Arca, et compare cette capsule à la zone pellucide de l'œuf́ des Mammifères. Chez Spondylus, la capsule manque; mais dans tous les cas le vitellus est entouré d'une membrane vitelline propre. Chez Dentalium, le même auteur décrit la coque qui entoure le vitellus pondu, la même qui entourait déjà l'ovule mùr dans l'ovaire. Le vitelluss n’a, du reste, pas de membrane propre. La coque est pereée d’un micropyle qui répond au point d'attache de l'ovule à la membrane du follicule de l'ovaire. De Lacaze-Duthiers fait observer qu’il en est de même chez Unio. Keber (Lxxix) a montré que ce micropyle est fermé chez les Naïades par un corpuscule, qu’il prend à tort pour un spermatozoaire. Von Hessling (xcvis) relève cette erreur et va jusqu'à nier l'existence de ce corpuscule, ainsi que celle d'une membrane vitelline propre, rue par Keber. O. Schmidt (cxiu) rélablit l'existence du corpuscule micropylaire et d'une membrane ritelline. Flemming (cxun) enfin, dans un travail très-soigné, nie positivement l'existence d'une membrane vitelline propre, et établit que le chorion est la seule membrane ritelline de l'œuf des Nä̈ades.

Le différend ne porte, comme l'on voit, que sur les réfinitions. Le chorion des Lamellibranches se forme, comme la membrane vitelline des Céphalophores, par une différenciation de la surface du vitellus. Seulement le vitcllus des Céphalophores est entouré ensuite d'un al bumen el d'une membrane. prodnits de la sécrétion des glandes accessoires, et sa membrane vitelline disparaît dans la plupart des car.s. Elle persiste au contraire chez les Lamellibranches et acquiert une foree suflisan!e pour jouer le même rôle physiologique que les membranes de l'ieuf des Céphalophores. Génétiquement parlant, le rhorion des Lamellibranches n'est done autre chose qu'une membrane vitelline 
très-développée, qui ne peut en ancune facon se comparer aux membranes de l'œuf des Céphalophores, et ne troure son homologue que dans la membrane temporaire qui entoure l'ovule non fécondé de ces animaux. La question de savoir si le vitellus des Lamellibranches s'entoure dans certains cas d'une seconde membrane vitelline plus mince et plus arlhérente, reste ouverte, mais ne saurait modifier cette manière de voir. Si, au contraire, on laisse de côté le point de rue morphologique, pour ne considérer que le rôle physiologique des membranes pendant le développement, l'on derra, avec de LacazeDuthiers, refuser à la membrane de l'œuf' des Lamellibranches le nom de membrane vitelline, et réserver ce terme pour la membrane qui paraît exister dans certains cas autour du vitellus lui-même.

Le nucléus de l'ovule des Lamellibranches est de consistance plus ferme que le protoplasme et renferme mou deux nucléoles. Le nucléole double a été décrit par Flemming chez les Naïades; dans ce cas, l'une des moitiés est plus petite el plus réfringente que l'autre et l'cntoure chez Tichogonia polymorpha comme un bonnet posé sur la tête.

La disparition du nucléus avant la sortie des corpuscules de direction a été constatée par Lovén (LXXi) et surtout par Flemming (CXLII). Davaine (uxxyur) rapporte que chez Ostrea il a, à cette époque, rencontré parfois un nucléus, mais que d'autres fois il l'a cherchć en vain.

L'acte même de la fécondation, la pénétration du spermatozoaire dans l'ovule, n’a été observé jusqu’à présent chez aucun Mollusque.

\section{LES CORPUSCULES EXCRÉTÉS.}

La sortie de ces corpuscules du vitellus a été signalée par van Beneden (xuII) pour Aplysia, puis par van Beneden et Windischmann (xLIv) chez Limax, si toutefois ces auteurs n'ont pas confondu la sortie de ces corpuscules avec l'expulsion de mucosités qui a lieu à plusieurs reprises pendant la segmentation de l'œuf des Pulmonés, el que Dumortier (xxxıı) avait déjà vue.

Von Nordmam (Lui) décrit la sortie de plusieurs corpuscules chez Tergipes, au nombre de deux à huit. Il confond donc les corpuscules excrétés avec les parcelles du vitellus qui se détachent pendant la première période de segmentation, et qui se courrent de cils vibratiles, ainsi que l'a très-bien décrit Selenka (Cxxxy). Ces parcelles vitellines détachées el nageant au lasard dans l’albumen de l'œuf, à l’aide de leurs longs eils. ont été prises par Nordmann pour des parasites. 
auxquels il donne le nom de Cosmella. C'est à tort que C. Vogl Lxul) identifie ces Cosmella avec les cellules convertes de cils moteurs du bord du voile, qui se détachent lors de la métamorphose, el présentent à peu près le mèmo aspect que les portions détachées du vitellus de Tergipes. Les corpuscules d'excrétion, dont jai constaté la présence chez Actreon (Elỵsia), on échappé à l'attention de Vogt. J. Reid (Lxiv) les déerit très-bien chez direr's Gymnobranches. Pouchet (xxxvu) passe à tort pour avoir vu ces corpuseules chez Lymnux . Cet observateur a chauffé des œufs fraichement pondus au foyer d'in microscope solaire, et étudié les phénomènes pathologiques quils présentent, phénomènes qui n'ont rien de commun avec les procédés physiologiques.

Fr. Mïller (cxix) est le premier auteur qui accorde me attention toute spéciale à ces corpuscules, qu’il considère comme des résicules, et auxquels il attribue une influence énigmatique sur la direction des premières lignes de segmentation, ainsi que l'indique, du reste, le nom qu'il leur domne, de Richtungsbläschen, soit résicules directrices. H. Rathke (Lxx), d'après des observations faites che\% Lymnous, Planorbis et Paludina, combat cette opinion et attribue la sortie de ces corpuscules à une rétraction et une diminution de volume du vitellus. Il montre qu’ils sont solides et nou pas creux, qu'ils sortent du vitellus l'un après l'autre, et attribue la constance qui s'observe dans leur point de sortie, comparé aux segmentations, à ce fait que İexpulsion de matière doit aroir lieu au point de moindre résistance, cest-à-dire à l'endroit où se montre le premier sillon du fractionnement. Il leur refuse avec raison toute influence, polaire on autre, sur la direction du sillomnement. Lovén (Lxis) décrit avec son exactitude bien connue les phénomènes que présente l'oruf fraichement pondu de quelques Lamellibranches. Chez Cardium parum quelques aufs pondus possédaient une vésicule germinative à moitié grande comme celle de l'ovule avant la fécondation et renfermaient enrore le nucléole. Cette vésicule change de forme, se ratatine et disparaît, tandis que le nucléole arrive à la surface du vitellus, et en sort pour former le corpuscule de direction, qui se divise souvent en deux. Il en est de même chez Modiolnia marmmata, où ce corpuscule soulève en sortant la membrane vitelline, dans laquelle il reste logé. De Quatrefages (cxxı1) a observé chez Teredo des wufs fraîchement pondıs fui possédaient encore la vésicule de Purkinje et la tache de Wagner; la tache disparaît la première, puis la vésicule parait 
pétrie dans diverses directions ef disparaît à son tour; il n'en reste qu'un espare claib occupant le centre du ritellus. Cet espace clair se divise en deux parties. lont lune arrive a la surface et en sort, formantle globule excrité, landis que la partie qui est restéc au centre se disperse dins le ritellus ef disparaìt. 11 est à noter que ce savant distinguc a em observer que le premier sillomnement se montrail au pôle opposé à celui où se trouve le corpuscule; cette observation demande confirmation. - Teydig (Lxiv) et la plupart des auteurs suhséquents considèrent, comme liathke, le corpuseule excrété comme une simple goutle de substance vitelline, chassée par des causes inconnues, mais qui n'a ni ne pent aroir d'influence sur la segmentation. Warneck (Lxy) décrit comment la tache claire qqui n'est pas une résicule) occupant le centre du vitellus s'allonge et se divise en deux parties, dont l'une arrive à la surlace et en sort en partie sous forme d'un, puis deux et parfois trois corpuscules. Après quoi, ce qui reste de la tache claire superficielle se rapproche de nouveau de la partie centrale, et toutes deux deriennent de véritables nucléi. Ces deux nucléi redisparaissent encore avant la première segmentation. D'après Koren et Danielssen (Lxxx, supplément) le vitellus pondu de Buccinum possède une résicule germinatire aree tache germinative. La résicule disparaît, et la tache sort, formant le corpuscule de direction: il repousse devant lui la membrane vitelline, dont l'existence so troure ainsi démontrée chez ce Gastéropode. Carpenter (cvi et cxin) ne tronve de membrane vitelline et de corpusenle de direction que chez ceux des vitellus qui se développent et qu'il nomme de rais mufs.

De Lacaze-Duthiers (cxu) a vu, ehez le Dentale, le vitellus présenter après la fécondation de petits mamelons à un pôle; unc petite quantité de substance granuleuse sort en ret endroit et se disperse dans l'albumen. Les globules de direction sortent ensuitc au pôle opposé à celui où se tronvaient les mamelons; ct cette sortie a lieu aussi, quoique d'une manière moins rógulière, ehez des ritcllus qui n’ont. pas été fécondís. Flemming (cxuli) enfin montre que chez les Naïades lo vitellus adhère au mirropyle, qui se troure ainsi bouché; le corpuscule excrélé sort du ritellus au pôle opposé au micropyle. Cic corpuscule est d'une rousistance plus ferme que le vitellus ou mème que le uucléus de l'ovule aranl la fécondation; il se divise en denx. Penrlant tout ce temps, le vitellus est dépourvu de vésicule ef de tache germinatires el prósente seulement une partie claire à son centre. lae corpusende no pent Inne être ni le unclécle ni la résicule gerni. 
native, qui n'existent pas au moment où ce corpuscule se forme; mais il est possible quil suit composé de la substance qui constituait ces éléments histologiques. Pendant la sortie du corpuscule, le vitellus présente des mouvements sarcodiques et semble pétri tantôt dans un sens, tantôt dans l'autre : le corpuscule croît pendant chacun de ces mouvements. Flemming pense que ces corpuscules doivent aroir une certaine importance, à cause de la constance arec laquelle ils se présentent chez des animaux de direr's embranchements. Je ne puis que souscrire à celle conclusion, tout en faisant observer que rien ne prouve quils aient une action polaire.

Il pent être important pour le vitellus de se clébarrasser de certaines matières devenues superflues; et la sortie de ces matières peut avoir lieu en un point déterminé et constant, sans que nous soyons obligés d'y voir autre chose (qu'une simple excrétion.

\section{LA SEGMENTATION.}

11 peut sembler incroyable qu'un observateur soigneux ait pu suivre le développement d'un ceuf, sans s'apercevoir que le vitellus commençait par se diviser et se rẹdiviser, pour donner naissance à la forme pluricellulaire ou framboisée. Et cependant tous les auteurs du commencement de ce siècle montrent clairement, par leurs descriptions, que ce procédé leur a complétement échappé. Aussi n’est-il pas bien étomuant de voir la confusion qui rògne dans les travaux de Carus, de Chatrefages (xxm) el mème de Dumortier (xximl) entre les cellules véritables, produits de la division du vitellus, et les globules nutritifs, qui remplissent ce dernier avant et après la segmentation. Carus (xxi) a eepentant représenté assez fidc̀lenıent un ritellus segmenté, et Dumorlier représente un des premiers stades du fractionnement, et eroit y voir la preure que l'embryon passe par l'état radiaire arant d'arriver à I'état bilatéral.

Sars (xxiv) est le premier qui ail observé la segmentation chez un Mollusque (Tritunia): il la décrit plus lard avec un peu plus de détails (xul). Van Beneden et Windischmann (xur) Ia mentionnent chez Limax. Von Norlmamn (L11) rapporte pue ehez Tergijes les divisions en deux et quatre sont l'ćgulières, mais que les suivantes sont très-irrégulières, jusqu’ì lat forme framboisée; données qui ont été confirmées pour une autre espèce du mème genre par Selenka (cxxiv). D’après le premier de ces deux auteurs. il sortirait du vitellus de Tergipes. au 
monent où il alleint la forme frambosér, un ghlobe très-rélringent qui ne tarterait pas à se dissondre. Cette dernière observation n’a pas été confirmée.

C. Vogh (Lxu) est, après liölliker, le premier anteur traitant du développement fles Nollusques, qui ait franchenent combattu les théories, encore assez répandues à colte époque, le téveloppements centrifuges ou centripètes, de multiplication endogénétique des cellules. Il n’a cependant pas constatí la disparilion du nucléus pendant la division de rhaque sphérule de segmentation, et šimagine au contraire que le nucléus persiste, et reste immobile dans l'une des deux moitiés rle sphérule, le vitellus seul se divisant en vertu de son activité propre. Ce bon observateur a fort bien vu ef lécrit le stade, si répandu chez les Céphalophores, oì l'óbauche emloryomnaire se compose de quatre grosses cellules opaques et de quatre petites collules transparentes posées en croix sur les premières. Ces dernic̀res apparaissent comme des mamelons, qui se détachent ensuite des grosses sphères. Les sphérules opaqu's so divisent ensuite, et deriennent anssi petites que les premières, font en formant un amas plus considérable et moins transparent. Vogt remarque qu’en ce point de développement les sphérules de segmentation sont devenues de réritables cellules, munies d'un nueléus, d'un protoplasme el d'une membrane, qui résulte d'une condensation de la couche la plus externe du protoplasme.

Kölliker (LXY) prétend, conformément à la définition que l'on donnait au mot cellule, que les sphères de segmentation ne sont pas des cellules, puisequ'elles sont dépourvues de membrane propre chez Doris, Lymnceus, Succince, elc. Il soutient arec raison, contrairement à l'opinion de Reichert, que les sphérules se multiplient par simple tivision; mais il se trompe lorsqu’il prétend que la division de la sphérule est précédée sle la formation endrgòne le deux nouveaux nucléi dans l’ancien nucléus. Reichert se rapprochait beaucoup plus de la vérité en soutenant que le nueléus disparaissait pour cotre remplacé par deux autres

Fr. Miiller (Lxix) montre que les premières segmentations ont pour centre le point où se trourent les globules de dirertion, et que ce point répond à celui nì se rencontrent les quatre petites collules transparentes du vitellus divisé en huit.

Lovén (Lxxi) a décril arec une grande prócision la segnmentation ehez Cardimm et Morludarin. Avant chaque segmentation les noyaux risparaissent, pour reparailua ares la segmentation dans lé nourelles 
sphères. A l'inrerse de ce qui s'observe chez la pluparl iles Nollusques, c'est la partie la plus riche en protoplasme qui prend la teinte la plus sombre. Plus tard celte relation se trouve renversée ef les cellules qui forneront la couche interne deviendront plus foncées que les autres. Le vitellus se divise en deux sphères inégales, la ligne de division colrespondant au corpuseule de direction. Puis la plus grosse des deux sphères se divise à noureau en une sphère proloplasmique qui vient prendre place à còté de la première, el une sphère plus transparente qui occupe le pôle opposé à celui oì se trouve le colpuscule excrété. Les deux cellules sombres se divisent ensuite en quatre, qui se rencontrent sous le corpuscule de direction, puis en huit. La cellule inférieure n'a pas de nucléus pendant toul ce temps; elle finit par s'en munir, au moment où les cellules supéricures se sont déjà beaucoup multipliées et l'enveloppent presque de loutes parts. Le corpuscule de direction se détache à ce moment, qui répond à la forme framboisée. et lombe. Après chaque segmentation les produits de la dernière division se rapprochent l'un de l'autre et se fusionnerạient à nouveau. Malgré l'exactitude de l'observateur suédois, il est difficile de ne pas croire (püil s'est fail illusion et qu'il a pris un rapprochement apparent, tel que l'a décrit plus lard Warneck, pour une fusion réelle. Lorén est le premier qui ait bien neitement montré que les nucléi ne sont vicibles que pendant les temps de repos, et disparaissent pendant les moments de division, et que le protoplasme éprouve un changement moléculaire, puisqu'il devient sombre pendant les moments oì les nucléi sont visibles, et transparent pendant lespériodes de travail.

De Quatrefages (LXX111) ronfirme pour Teredo quelques-mes des domnées de Lovén, puisqu’il a vu le vitellıs se diviser en deux sphères parfois égales, parfois inégales. dont lune seulement se divise rapidement et enveloppe l'autre.

Warneck (LXxy) a montré que chez Limax el Lymmeus le mucléus perd avant chaque segmentation ses contours nets, et se lransforme en une tache claire, qui s'allonge et se divise, le reste du ritellus suirant le mouvement. Après la division, la tarhe claire de chatrune des nouvelles sphères reprend des contours plus nets, et redevient un réritable nucléus, qui est d’abord petit, mais croît rapidement jusqu’à son rolune normal.

Le premier sillon part du point oì se troment les corpuseules excrétés ; à mesure que le sillom s'approfondit, dans re slade comme dans les suivants. il prend me direction oblique, an sorte qur les 
sphères en roie de formation paraissent chevaucher l'une sur l'autre. Apròs chaque dirision, les deux sphères qui viennent de se séparer l'une de l'aulre se rapprochent à nouveau et entrent en contact intime. Leur ligne de démarcation est formée par de la substance claire qui se change en une véritable vacuole; celle-ci déverse son contenu à l'extériemr, c’est-à-dipe dans l'albumen, où il se disperse, ef cela au pôle opposé à celui où se tronvent les globnles de direction.

Pendant les temps de repos, l'ensemble des sphires de segmentation prósente me forme arrondie; arant et pendant la segmentation, rhaque sphérule se ramasse et forme une bosse ì la surface de l'ébauche embryonnaire. Warneck décrif arec la plus grande précision toutes les dirisions successires jusqu'à la forme framboisée. Le nombre des sphérules ou cellules ra en augmentant en progression géométrique jusqua u chiffre de quatre, après quoi la progression devieut arithmétique. Ces quatre cellules se divisent en quatre grosses el quatre pelites, qui alternent aver les premières, et occupent le pôle marqué par les corpuscules excrélés. Puis les quatre grosses cellules se divisent à nouveau, produisant quatre cellules claires, yui s'interposent obliquement entre les quatre grosses cellules et les quatre pelites cellules du stade précédent. Ce sont ensuite ces demières petites cellules qui se fractionnent, puis les grosses cellules se divisent pour la troisième fois, et enfin les quatre petites cellules les plus roisines des corpuscules de direction se fractionnent amssi une troisiène fois. L'ébanche embryonnaire a atteint, de la sorte, la forme framboisée, et dès lors les cellules s'entourent chacune d'une membrane et leurs divisions ultérieures sont chaque fois précédées de celle de leur nucléus.

Koren el Danielssen (Lxx) ont prétentu que chez. Buccinum et Pupura les vilellus, au lieu de se segmenter, se réunissaient ensemble en paquets composés chacun d'une centaine d'cufs, et que chaque paquel donnait naissance ì un embrỵon. Carpenter (Cvi), dans un trarail fait en collaboration avee .1. Busk, a démontré que ces Prosobranches ne font pas exception aux lois de la nature. Les pontes ne renferment qu'un petit nombre l'orufs féconds, qui se segmentent d'une manière régulière et se transforment en embryous, el ces embryons dévorent sinplenent le reste les ceufs, qui se sont fractionnés très-irrégulièrement.

D’après Davaine (Lxxvur), le vitellus eu voie de segmentation chez Ostrea tiendrait le milieu entre la forme que présentent les Céphalophores et celle des Lamellihranches de Lorén. Eu effel, dans le stade 
à quatre sphères de segmentation, ces sphères seraient tantòt sensiblement égales entre elles, tanlôt l'une serail beaucoup plus grosse que les trois autres. De mème, an stade suirant, il y arait parfois quatre grosses cellules foncées et quatre petites placées en croix sur les premières; mais plus sourent l'unc des grosses cellules l'emportait de beaucoup en rolume sur les sept autres. Ces différences s'efficent lorsque l'embryon a alteint la forme framboisée.

Gegenban (c1) a suivi le développement de l'seuf chez Hyalau Cleodora et Pterotrachera. Le vitellus fraichement pondu se compose d'un stroma renfermant des globules ritellins également répandus dans toute son élenduc. Il serait muni d’un nucléus dont la division précéderait toujours celle de la sphère de segmentation. Evidemment cet auteur si eonsciencieux s'est laissé tromper ici par les apparences et a pris la portion purement formative du vitellus pour le nucléus, ainsi que je le démontrerai dans le courant de ce mémoire. Le vitellus se divise en quatre sphères, dont trois sont composées de substance nutritive et la quatrième de substance furmative, observation qui n'est quapproximativement juste. Puis la cellule formative se divise rapidement el produit un amas de petites cellules claires, qui enveloppent petit à petit les trois cellules nutritives; ces dernières restent entièrement passires pendant toute la première partie du déreloppement. Nous rerrons plus loin que celte description ne renlerme qu’une partie de la rérité et que les cellules untritires sunt presque aussi actives que les autres. Nous rerrons aussi que lil quatrième cellule primitive des Ptéropodes, la grosse cellule protoplasmique, n'est pas la seule qui produise la conche externe, et que sous ce rapport les rufs des Pléropodes à corquille droite se rapprochent bien plus quil ne pourrait sembler au premier abord des neufs des autres Cóphalophores. Chez Hyalea tridentata (je ne sais quelle est l'espèce que Gegenbaur désigne de ce nom; elle diffère, comme on le verra, lant par la trinte de son vitellus, qui est incolore, que par le développement de son ouf de la Cacolivin tridentata [Hyaliea tridentata], Lamark, dont j’ai étudié l'embryogénie), chez Hyalcea (sp.?), dis-je, ce sarant distingué a vu les aufs se dérulopper tantòl de la même manière que chez les autres Hyaléacées et tantòt d'une façon assez différente. Dans ce dernier 'as, en eflel, le vitellus sc divise en deux cellules très-incigales, dont l'ume, composéc de protoplasme. se dirise rapidement ef enreloppe la grosse cellule nuiritive, qui se comporte passivement; ou bien le vitellus se dirise en denx cellules très-inégales. 
dont la plus grosse domne aussitòt naissance à deux cellules. L'une de ces dernières se divise rapidement el enveloppe les deux autres grosses cellules restées passives. Ces divers modes de développement se rencontreraient l'un à còté de l'autre dans me mêne chaine d'œul's et ces différences n’influent en rien sur la suite du développement. Je regrette d'autant plus de n a aroir pas rencontré l'espèce qui a servi aux recherches de Gegenbaur, que des anomalies aussi curieuses et intéressantes ne se sont rencontrées rhe\% ancun des genres que j’ai étudiés.

Chez Deutalium, d'après de Lacaze-Duthiers (cxir), le vitellus se divise soit en deux, puis quatre sphères égales, soit en une petite et une grosse sphère qui se scinderait du coup en trois. Les sphères sont opaques et présentent au centre un espace clair; puis ces quatre sphères se divisent en huil, dont quatre grosses et quatre pelites, comme chez. tant de Céphalophores. Les petites cellules se divisent plus vite que les grosses et les enveloppent; mais la couche périphérique est tout aussi opaque que la conche interne. Le mode de lormation des quatre petites cellules est une sorte de bourgeonnement.

Cet observateur soigneux montre jue che\% Vermetus (cxim) le vitellus se scinde en quatre, et nons apprend i cette occasion que che\% Bullepa la division en quatre sphères s'opère d'un seul coup; ces sphères continuent à se diviser juscu'an nombre de six ì dix. Alors apparaît, d'un côté. un amas de sphérules, qui serait le résultat d'une sorte de croissance se faisant sur un des côtés de l’œuf, et non le résultat du détloublement des sphérules existantes.

Les sphères vitellines (nutritives), qui sont d'abord latérales, sont englobées ensuite par une couche transparente, qui a son point de départ dans l'amas des sphérules transparentes.

Claparède (cxin) montre que chez Neritina tous les vitellus renfermés dans ume capsule se segmentent, mais qu'un seul se développe et devient un embryon qui dévore les autres wufs arrêtés dans leur développement.

Von Hessling (cxxi) tigure un vitellus de Unio divisé en huil sphérules égales, observation évidemment erronée, ainsi que d'autres l'ont démontré.

Keferstein et Ehlers (cxxvis) décrivent la segmentation d'une $A E O$ lis de la manière la plus superficielle.

Lereboullet (cxxw11) étudie avec grand soin lat segmentation chez Lymmerus; ; son exposé concorde en somme arec celui de Warneck. Il y 
a cependant quelques petites différences. Ainsi Lereboullel croit voir dans le vitellus, après la sortie des corpuscules de direction, quatre mucléi distincts, qui redisparaitraient avant la première segmentation. Il indique très-clairement la disparition du nucléus en tant qu'élément morphologique avant ehaque division de sphérules, et insiste sur les modifications qu'éprouve le vitellus, el qui se répètent toujour's les mêmes à chaque segmentation successive. En effet, la substance vitelline est composée en majeure partie de granules solides que l'auteur nomme les granules plastiques, et qui ne se montrent sous celte forme que pendant le travail de division. Après la segmentation, et lorsque les nouvelles sphérules se rapprochent à noureau l'une de l'autre, ces granules deviemnent ehacun une petite vésieule qui renlerme un petit grain brillant et ces vésicules sont désignées du nom de vésicules plastiques. Lorsque l'embryon arrive à la forme framboisée, les sphérules prennent tous les caractères de véritables cellules et leurs cléi renferment de petites vésicules. Enfin les nucléi finissent par se diviser, au lieu de disparaitre, avant chaque division de cellule, et renferment alor's chacun un à deux nueléoles.

Stuart (cxix) décrii la segmentation chez les Aplysiens; il constate l'absence de nueléus pendant les segmentations, mais les réapparitions passagères de eet élément lui ont échappé. Le vitellus se divise en deux sphères égales, qui se divisent à leur tour chacune en une grosse sphère opaque nutritive et une petite sphérule formative. Ces deux petites sphérules se multiplient et enveloppent les deux grosses sphères, qui restent passives. A cette époque sortiraient du eôté formatif les "denx corpuscules de direction constants " (sic). D'autres masses vitellines peuvent aussi se détacher de la surfice et se courrir de cils vibratiles. Stuart remarque qu'après la formation de l'amas de sphérules périphériques d'autres sphérules pareilles se montrent indépendamment de cet amas au pôle opposé et finissent par entourer de loutes parts les deux sphères centrales; toutefois il ne précise pas le mode de formation le ces cellules périphériques.

Selenka (Cxxxr) décrit le vitellus de Tergipes claviger comme composé de protoplasme granuleux et de corpuscules de consistance céreuse qui se dissolvent pendant la suite du développement. La segmentation est irrégulière, contrairement à ce qui sobserve chez les autres Mollusques. Cette irrégularité est iei la règle, et de cette ébauche irrégulière sort un embryon parfaitement régulicr. Pendant la segmentation, des parcelles se délachent du vitellıs, ainsi que Norhnann l'a 
léjà vu (Cosmella); ces pareelles peuvent être si nombreuses que la masse formative en est réduite dr moitié. Et pourtant le petit embryon qui en résulte est pareil te tous points aux embryons qui se sont formés de la presque totalité de la substance du vilellus! Au lieu de rester attachées les unes aux autres, les sphérules de segmentation sont libres el ne sont retenues que par la membrane de l'oul. Eilles se resserrent, lorsqu'elles ont atteint le chiffre de deux cents on trois cents, pour constituer la forme framboisée de l'embryon. Toilà certes des faits dignes d'attention!

Chez Purpura lapillus (cxixvi) Sclenka confirme les données de Ciarpenter sur les deux espèces d'wufs que renferment les capsules. Les uns, en petit nombre, sont dépourvus de nueléus el se divisent régulièrement, tandis que les autres ont un nucléus, se divisent d'une manière fort irrégulière; et n'ont d'autre destination que celle de servir de nourriture aux embryons sortis des premiers. L'autenr se demande si les vitellus féconds n’expulsent pas leur nucléus de leur sein ? La chose n'a pas été observée directement. Les rufs féconds se divisent en deux sphères, dont l'une, plus grosse, composée de matière nutritive, et l'autre, plus petite, et formée de protoplasme. Les corpuscules de direction sont à la limite entre ces deux cellules. La plus pelite des deux sphères se divise rapidement et entoure la grosse sphère, qui resterait cntièrement passive.

Salensky (cxxxvi) déclare quele vitellus de Calyptráa sinensis se développe de la façon bien connue pour les Gastéropodes, mais en donne en même temps une courte description qui ne concorcle guère avec le type bien connu. Tne de ses figures représente cependant pour Trochus varius la forme aux huit sphérules, clont quatre grosses opaques, et quatre petites transparentes; ces dernières se diviseraient plus vite, et leur's dérivés envelopperaient les grosses cellules, qui se multiplient entre elles.

Langerhans a suivi le développement d'Acera bullata (cxis). La description qu'il dome de la segmentation chez ce genre coïncide dans ses traits principanx avec celle de Stuart pour les Aplysiens. Les corpuscules de dircetion ne sont pas mentionnés.

E. Ray-Lankester (CXL) a vu les mêmes quatre cellules résultant des denx premières segmentations chez Aplysia, mais il l'ail dériver tout l'ectoderme de la subdivision des deux petiles sphérules protoplasmiques; observation évidemment superficielle.

Flemming (cxun)enfin décrit avectótail la segmentation chez les Ano- 
duntes. Après la surtie des corpuseules de direction, le vitellus se divise en une grosse splière opaque el une sphère plus petile el transparente; leur ligne de démarcation passe au-dessous des corpuscules excrélés. Un nucléus apparaît ensuite dans chacune des deux. Le stade suivant se compose d'une grosse cellule opaque et de trois petites cellules, que l'auteur fait dériver, sans l'avoir observée directement, de la division de la plus petite des deux cellules. C'est un point qui serait à démon. trer, ce me semble. Ces trois jetites cellules se diviseraient en huil et plus et formeraient un amas plus volumineux que la grosse cellule, fait qui peut bien donner à penser que la grosse cellule ne reste pas aussi passive que le eroit Flemming. La grosse cellule se divise alors en quatre, et c'est pendant ces divisions que l'auteur a nettement vu et figuré les étoiles moléculaires que jai découvertes antérieurement chez les Géryonides, les Ptéropodes el d'autres animaux, et qui président à la division, après la disparition du nucléus. L'auteur n’est pas arrivé à une notion claire du rôle que jouent ces éloiles et n'a pas suivi leur division. Leur importance au point de vue de la théorie cellulaire lui a clonc échappé. Il insiste du reste avec raison sur ce fait, que le nucléus, comme tel, disparaît pendant la segmentation, et que, quoique la substance qui constituait ect élément ne soit pas détruite, l'on ne peut pourtant pas dire que le nucléus persiste en réalité, " pas plus qu'un cristal de sel dissous dans l'eau n'est un cristal. :

\section{FOHMATION DES HEULLETS EMBRYONNAIRES.}

Ju laisse de côté les discussions sirr la formation de l'embryon par' fenillets et sur les développements centripète et centrifuge, qui n’ont g'uère qu'un intérêt humoristique (xxm et xxxı1), pour recueillir les renseignements positifs, que nous fournissent les auteurs relativement récents.

Sars (xuı) a ru l'embryon des Eolidiens prendre, après la forme framboisée, celle d'un fer à cheval.

Van Beneden et Windischmann (xuIv) décrivent et figurent l'embryon de Limace, après la segmentation, comme composé d"une membrane épaissie d’un côté el eniourant les cellules vitellines.

C. Togl (LxI), après aroir montré que l'amas de cellules claires, qu'il nomme périphériques, s'agrandit et ‘lépasse l'amas de cellules foncées, el cela " glàce ì un changomont progresif de cellules contuales en 
rellules périphériques n, fail roir que la masse des cellutes centrates s'évide el présente une fente, qu'il nomme la fente mamelomaire. Cette fente s'allonge el le discque périjhérique se rerourbe vers la fente en forme de lasse. Puis la couthe périphérique achève de se refermer par-tessus la masse centrale, et l'auteur incline à croire que la fente mamelonnaire n'est qu'un plissement de la couche centrale, dù à une compression de la part de la conche externe. C. Vogt est le premier ubservateur qui ail incontestablement vu el décril l'invagination primitive, mais sans en saisir toute la portée.

Lovén (Lxxi) décril et représente, dans des dessins extrêmement soignés, des embryons de Cardium et de Modiolaria composés d'une couche périphérique claire et d'une couche centrale, qui provient sans nul doute de la dirision de la grosse sphérule centrale de la période précédente. Liembryon est réniforme el présente une dépression de la couche périphérique; au fond de cette dépression se trouve une ouverture qui correspond à une ouverture de la couche interne. L'auteur croit que ce point est celui d'où le corpuseule de direction est tombé. L'ouverture se referme. La masse centrale, vue par le côté opposé à l'ourerture, forme deux lobes latéraux. Faut-il conclure de cette description que Lorén a assisté à une inragination sans la comprendre? Rien ne nous atutorise à le croire.

De Quatrefage's (cxxuI) at vu des embryons de Teredo composés de deux couches concentriques, mais sa description perd en clarté par le fait qu'il persiste encore à combattre la théorie cellulaire.

Gegenbaur (Lxxvm) a observé des embryons de Pulmonés, composés de deux feuillets. J. Nüiller (Lxxin) a vu des embryons de l'Entoconcha, dont le feuillet interne se composait en apparence seulement de quaire grosses cellules opaques; mais, en examinant de plus près, il a recomnu que chacune de ees sphérules possédait plusieur's nucléi. Leuckart (xсI) a vil chez firoloildes une fosselte correspondant an point où la conche périphérique n’a pas encore complétement enveloppé les grosses cellules centrales; celte région se renfonce jusqu'au centre de l'reuf. Il s'agit iei clairement de l'invagination primitive, que l'auteur considère comme l'origine du tube digestif. MoquinTandon (c) a ru et représenté cette invagination chez Ancylus, mais ne la décrit pas clairement. Gegenbaur ( $\mathrm{CI}$ ) décrit très-bien l'embryon des Ptéropodes orthoconques, composé d'un amas central de trois grandes cellules mutritives el d'une couche externe de petites cellules qui enveloppe la premiere de toules parts. Il a reconnu que res cel- 
lules centrales conservent curore lealrs lormes cxtérieures, à un moment où chacune est munie intérienrement le plusieurs nucléi, et croit aroir vu que chacune de ces cellules se divisait d'un comp en autant de cellules qu'elle renl'ermait de noyaux.

Lereboullet (cxxvin) décrit aree soin el en détail la formation de rleux feuillets chez Lymmous. Le germe pluricellulaire résultant de la segmentation s'aplatit un peu d'un côté et se renfonce au côlé opposé, formant une vaste et profonle cavité. L’anteur paraît admettre, quoiqu’il ne le dise pas d’une manière bien claire, que ce côté, qui s'inragine, répond à celui qui se compose de petites cellules; il resterait encore à savoir si ces petites cellules orcupent bien le pôle formatif : sur ce point les renseignements font défaut. Les bords de la cavité d'invagination paraissent s'enrouler sur eux-mèmes, les cellules qui formaient le rebord rentrant dans la cavilé. Ces bords se rapprochent par le milien des côtés opposés et réduisent ainsi l'ourerture d'invagination, la transformant en une fente allongée, qui se termine en avant par une petite ouverture ronde. Cette fente se resserre lentement el finit par se lermer el se souder ; clle répond au dos de l'enbryon. L'ouverture ronde persiste et devient la bouche. Au-dessous de cette ouverture, la couche externe est déjà épaissie dans toute une région qui devient le pied ; c’est celte position dn pied qui permet d'orienter l'ébauche embryomnaire arec tant de précision. Nes propres observations sur le développenent de Limax confirment en somme ces résultats importants, auxquels est arrivé l'illustre embryogéniste français.

Stuart (cxxix) rapporte que chez les Aplysiens, après la formation de la couche périphérique, les cellules centrales se rlivisent à nouveau et produisent par le mème procédé, en commencant par le pòle antérieur, qui eorrespondrait au pôle formatif, me seconde couche de cellules intermédiaire entre l'ectoderme el les cellules centrales. quelles enveloppent de toutes parts. Cette conche noyenne est moins transparente que l'ectodernue, mais moins oparque que le reste des cellules intérienres. Ces dernières se diviseraient ensuite en 1111 rertain nombre d’agglomérations, qui ne panaissent pas avoin, dans lopinion de l'antenr, d'autre destinfe que celle d'ètre résorbées par les antres tissus. Le tube inlestinal se formerait par une invagination de la conche externe ef de la rouche moyenne, la comehe externe donnant naissance à l'épithèle, la eonche moyemne aux muscles du tube digestif. les masses rentrales à du tissu eonjonctif. 
La description que domne Selenka (cxxivi) de la lormation des feuillets chez Purpura est fort singulière; elle est, du reste, accompagnée de dessins fort beaux. La couche périphérique provenant de la division de la plus petite des deux sphérules primitives envelopperait petit à petit la sphérule oparue, laissant cependant une ouverture au pôle opposé au pôle formatil. Aux bords de cette ouverture, qui deviendra la bouche, la couche périphérique se retrousserait el se mettrait à pousser en arrière entre la sphérule centrale ot la couche périphérique pour aller enfin se rencontrer et se refermer au pôle aboral. La sphère nutrilive resterait entièrement passire pendant ce temps, ne contribuant en rien à la formation de l'entoderme, dans la cavité duquel elle finit par se trouver. Elle se disloque en morceaux et sera simplement résorbée ou digérée. C’est le procédé désigné par l’auteur sous le nom d'épibolie, par opposition à l'embolie, qui est le cas le plus fréquent, lorsque le vitellus de nutrition est considérable. Je me borne à rapporter ces opinions, qui me paraissent dilficiles à admeltre.

Ganine (cxxxix) décrit chez Cyclas l'embryon comme composé d'une sphère cellulaire creuse dont les éléments, en couche unique, sout tous égaux. Puis ces cellules se divisent rapidenent sur l'm des côtés de la sphère, et cette couche de petites cellules vient englober l'autre moitić, composée de grosses cellules remplies de granules nutritifs. Arrivées ainsi dans l'intérieur, ces cellules nutritives se résorbent petit à petil. T.e fenillet externe primitif se scinde en un fenillet ectodermique el un mésoderme; en un endrnit, le feuillet primitif présente un épaississement considérable qui donne naissance à l'entoderme. Chez Lymmreus, Planorllis, Physa, après l'englobement des cellules nulritives, les trois leuillets se forment de la mème manière que chez Cyclas, et les cellules nutritives restent aussi passives et ne prennent part directement ì la formation d'aucun organe.

Langerhans (cxul) montre que chez Acera la comche externe, qui résulterait suivant lui de la subdivision des deux sphérules raires, se compose de plusieurs couches de cellules, au point qui répond à la position qu’occupaient res sphérules, ainsi qu'au point diamétralement opposé. Ces deux amas ectodermiques s'enfoncent par prolifération entre les deux splérules centrales, qui resteraient tout à fait passives. Chez une Doris, le même autemr'a vu la sphère rellulaire framboisée se renfoncer d'un côté et lormer ainsi une cavité d’invagination dont l'entrée se rétrérit el devient la bourhe. 
Ray Lankester (CXL) rapporte pour Aplysia des faits qui confirment le travail simultané de Langerhans relatif à Acerr. Les rleux espèces d'Aplysia étudićes par lanteur se comportent, dı reste, d'une nianière assez différente: che\% la grande espèce les sphérules centrales se divisent de bonne heure, ainsi que Stua!t l'avait déjì décrit arec plus de détail et de précision; chez la petite espèce les sphérules cen. trales conservent leur forme jusqu'au moment où elies sont complétement résorhées. Le même auteur mentionne l'existence déjà connue d'une invaginalion primitive chez les embryons de Gymnobranches, et l'a reconnue aussi chez Pisidium.

En résumé, la formation de l'entoderme parait se faire de différentes manières. Dans les cas où l'embryon se compose de bonne heure d'un grand nombre de cellules de dimensions peu direrses, l'une des moitiés, probablement la moitié nutritive de la sphère cellulaire, s'invagine dans l'autre et la couche invaginée derient directement le feuillet interne; l'onverture d'invagination marque l'endroit où se trourera la bouche. Dans le cas, au contraire, où la moitié nutritive de l'embryon se compose de cellules beaucoup plus grosses que les autres, ces cellules sont enveloppées par la couche externe sans qu’il y ait d'invagination proprement rite; mais ce type, si bien compris par de Lacaze-Duthiers, pour le Vermet, peut aisément se ramener an lype d’invagination que présentent les Hétéropodes et les Gymnobranches. Au lieu de rentrer dans la moitié formative du blastoderme, les grosses cellules sont enveloppées par lui, et la bouche marque, dans l'un comme dans l'autre cas, l'endroit où cet enveloppement s'achève. Les organes de la digestion, à l'exreption de l'œsophage. descendent directement de la couche enveloppée, ce que de LacazeDuthiers a nommé le blastème gastro-hépatique. Dans d'autres cas (Aplysiens, Cyclas, Pulmonés), l'entoderme paraît se former par un épaississement de l'ectoderme au pôle formatif, tandis que les cellules du pôle nutritif sont englolsées d'abord, puis résorbées sans donner naissance à aucun tissu. Ce type et celui de Purpura ne me paraissent pas pouroir ètre comparés aux deux premiers, du moins pas dans l'état actuel de nos comnaissances.

FORMATION ET DIFFÉRENGIATIUX DU TÜBE DIGESTIF.

La plus ancienne olyservation positive sur ce sujet (que je réussisse à trouver dans la littérature est celle de de Oualrefages (xxui) pour 
Plamorbis et Lymmers, d'après laquelle le tube digestil se forme par écartement des eellules élémentaires. Il ítahlit que les cavités de l'embryon ne naissent pas dans des cellules, mais sont entourées de cellules qui forment leurs parois. Che\% les Anodontes (xxvis), le même auteur a vi le foie et l'estomac se creuser de la même manière; il a vu l'estomac s'allonger etse couder, et fait remonter l'origine première du foie à une rangée de globules qui se trouve dans l'intérieur de l'enbryon. Laurent (xxvul) indique très-bien que l'embryou de Limace se compose de denx membranes, qui se continuen d dans les deux conches de la vésicule (céphalique). Plus tart, le même auteur (xxxi) observe avec justesse que la vésicule réticulée (feuillet interue de la vésicule céphalique) communique avec le canal intestinal. L’aspect réticulé de cette couche est dù à l'aplatissement mutuel des vésicules (cellules) qui la composent. D’après Jacquemin (xL), les globules centraux de l'embryon se mettraient bout à hout pour former l'intestin, et l'un d'entre eux dommerail naissance à la bonche. Sars (xuli) décrit le tube digestif' des lan'ves d'Eolidiens composé d'un a’sophage, d'un estomac muni de trois renflements qu'il considire comme l'origine du foie, el d'un intestin. Van Beneden ef Windischmann (XLIV) ne décrivent le tube digestif qu'au moment où la vésicule céphalique a déjà disparu; le pédicule vitellin fournit un prolongement qui se rend au bord droit du bouclier et devient l'intestin. Le canal alimentaire forme alors un simple tube, allant presquue en droile ligne de la bouche à l'amus, et sur lequel s’insère le sac vitellin. Au moment où se forme la première anse intestinale, les cellules dü vitellus (qui n'est autre que la couche interne de la résicule céphalique) se liquéfient, et l'on voit le liquide gramuleux, qui résulte de cette dissolution, chassé du citellus dans l'intestin, et de l'intestin dans le vitellus. Cetle observation, que je puis confirmer, est de la plus haute importance, puisqu elle montre clairement que le sac nutritil oceupe ici la mème position et joue le même rôle que chez les Ptéropodes. Jaurai ì revenir sur ce sujet dans un autre mémoire. Van Beneden et Windischmann déerivent encore comment le vitellıs (sac: nutritif, Ref.), inséré derrì̀re l'estomac, se liquélie de plus en plus, comment le tube intestinal forme des anses en s'allongeant, et disent enfin que la glande biliaire se forme au-dessus de l'intestin, aux dépens de cellules semblables à celles dı vitellus, ce qui explique l'erreur des auteurs, qui font provenir directement cetle glande du vitellus de l'embryon.

Alder et Haucock (u) notent que le tube intestinal des larves écloses 
de Gymnobranches est intérieurement garni de cils. J'ignore si ce fail a élé observé précédemment; je ne le crois pas.

Ton Nordmann (Lui) déerit le tube digestif de larves toutes formées de Tergipes, comme composé d'un estomac relié à la tête par une masse cellulaire, avec intestin peu distinct et portant sur le côté le foie granuleux. Au moment où la larve se prépare à subir la métamorphose, l'xesophage et l'intestin, qui ra déboncher à còlé de l'otoryste de droite, sont devenus distincts; l'estomae est vilić intérieurement et porte à sa partie supérieure les deux lobes jaunàtres du foie, dont le plus gros se trouve à gauche. Après la métamorphose, il se lorme sur le dos des prolongements de la peau, dans lesquels pénètrent des diverticules du tube intestinal. A quel point ces diverticules sont-ils comparables an foic? Il est difficile de le dire; mais les denx lobes que ron Norlmamn et la plupart des auteurs subséquents considèrent comme l'origine du foie, correspondent éridemment anx sacs nutritifs des Ptéroporles.

Sars (Lvu) corrige ses dommées précédentes sur les larves de Gymnobranches, en ce sens qu'il u’y a que deux lobes du foic an lien de trois, et que l'estomac est garni de eils ribratiles.

C. Vogt (Lxu) décrit comment la couche externes de l'embryon presente unc dépression située entre le pied et le bord du voile, dépression qui marque l'endroit où la couche périphérique s'est refermée, et ćnonce clairement l'opinion que le point où se trouvaient les quatre pelites cellules primitives (pôle formatif), correspond an sillon qui sépare le voile dı dos de la larve. Ensuite, la masse rentrale de la larve se groupe en deux agglomérations latérales, et au milieu se troure toujours la cavité yui provient de la fente mamelonnaire (invagination primitive) et qui est également enveloppée par la masse cellulaire centrale. Des deux agglomérations latérales de la masse centrale, celle de droite, qui est de dimensions plus considérables, devient l'estomac arec l'intestin; celle de ganche, le foie. Ce foie forme une nrasse compacte de grosses cellules, qui s'étend depuis le pied jusqu'au fond de la coquille; ces cellules se multiplient quelque peu et se remplissent de gouttes de graisse. L'extrémité de l’intestin n’entrerait en communication avee l'extérienr que vers la fin de la vie larvaire. L'iesophage ne se lorme qu'après l'estomar el entre en communication aree lui; l'autenr, insiste sur le fait que la bouche répond à la fente mamelonnaire, mais que l’asophage n'est pas dù à la persistance de ceite fente, que le derme se referme par-dessus la fente pour se 
creuser ensuite el former l'nesophage (LxI, p. 67 el 68). Les parois Iu tube digestif se scindent en un épilhèle vibratile, dont les cils battent tous dans la direction de l'estomac, et en une envoloppe muscularire. les éléments histologiques cessent ensuite d'être distincts. Le foic se ereuse par la fusion de sa partie centrale; il est cilić et rommunique avee l'estomac; les particules nutritives sont chassées de l'un dans l’autre, el vice versú.

J. Reid (cxv) rapporte des observations smr les genres Doris, Goniodoris, Polycera, Dendronotus, Doto ef Enlis, qui viennent, en somme. confirmer les observations bien plus soignées de Vogt. Il a vu che\% tous ces Gymnobranches deux sacs vitellins qu'il nomme des cellules, ot qui sont accolés à la partic supérieure de l'estomac, l’un, plus gros, à gauche, répondant an foie de l'Actéon, l'autre, plus petit, à droite.

Lovén (cxxi) montre que, ehez Cardium, la masse des cellules oparjues de l'intéricur se différencie d’abord en une masse centrale. estomac et foic, el en deux trones parallèles, esophage el intestin. Ces partics se creusent ensuite par l'écartenrent des collules qui les composent; la bouche ne perce que beaucoup plus tard. Le foie se compose de deux lobes placés sur les deux côtés de l'estomac. Le tube digestif est garni de cils qui battent dans la direction de l'estomac. Cette deseription peut aussi s'appliquer au genre $M$ ontacuta, chez lequel lanteur a vo lo foie, d’abord solide, aree des nucléi disséminés dansa masse, se creuser ensuite et se composer alors d'espaces polyédriques sclrés les uns contre les autres (cellules sans doute). Lil cavité de re foie communique avec celle de l'estomac. cl les deux organes se renvoient alternativement leur contenu. Chez des larves de Lamellibranches beaucoup plus avancées, mais d'espèces indéterninées, le loie est rert el se eompose f'une multitude de petits sacs.

Iax Schultze (txxis) rapporte que le jeune tle Tergipes lacin., après la métamorphose, a déjà des mandibules, mais n’a pas encore du tube intestinal. Malgré l'exactitude bien commue de cel excellent observateur, il est permis de croire que l'opacité de l'animal est la seule cause de ce résultal négatif. Plus tard, il a distinguć ces parties. ainsi que deux ou trois caecums de la paroi de l'estomac; les prolongements du dos de l'animal se composent, dès l'origine, d'un cacum de l'estomac et d'une duplicature de la peau.

be (huatrefages (cxxin) rapporte que le tube intestinal te Teredo se forme par le crensement l'une masse gramulense arroudie qui donne 
naissance au foie, ef d'une masse granuleuse allongée qui donne naissance à l'intestin. Ces deux masses se tronvent près de la char. nière.

D’après Leydig (cxxiv), il se forme unc carité dans l’intérieur chez l'embryon de Paludina, et cette cavité entre en communication arec deux enfoncements venant de l'extérieur, et dont l'un représente l'resophage ef l'autre l'intestin. Cette dommée fait penser à relles de lenckart et de Lereboullet, dont il sera question plus loin. L'estomac. est entouré de cellules assez grandes et réfringentes, qui se retirent d'un còté pour former le foie. Puis l'anus est repoussé en avant par la croissance de la coquille, l'estomac s'allonge et se recourbe; des globules de graisse apparaissent entre les cellules du foie. Cet organe prend une forme lobée, puis lolliculaire, et s'entoure d'une tunique propre.

0. Schmidt (Lxxy) a ru, ehez Limax ayrestis, le sac ritellin faire hernie par la nuque, puis rentrer petit à petif. Il n'a réussi à voir le tube intestinal que bien longtemps après sa formation, ainsi que cela résulte d'une comparaison de son travail avec ceux de Laurent el de van Beneden et Windischmann, et ne sait si le sac vitellin eommunique avec le tube intestinal; il affirme que ce sac deviendra le foie, sans en donner la preuve.

Gegenbaur (Lxxrit) a vu chez Limax les grosses cellules réfringentes. entourées par la couche extcrne, faire saillie d'abord à l'extérieur, et rentrei ensuite dans l'intérieur. Puis apparait l'msophage par enfoncement de la couche externe ; lintestin se forme, de son còté, par écartement dans un groupe de grandes cellules situées en arrière du bouclier, et seulement alors la communication s'ourre entre ces deux cavités. La masse vitelline ne prend pas la moindre part à ces formations: elle est repoussée, et se troure ensuite entourée par les anses intestinales. L’esionac se tapisse de cils, l'intestin débouche à J'extérieur. Du milieu du gros intestin naît un cul-rle-sac dirigé en arrière, qui ne tarde pas à redisparaître. La mandibule supérieure se forme par le durcissement de cellules épithéliales. L'auteur décrit, arec son exactitude habituelle, les moditieations qu'éprourent les grandes cellules centrales. Elles ront en augmentant ef se multipliant avec le développement de l'embryon, et se' remplissent chacune d'une masse très-réfringente, qui repousse le contenu de la cellule et son nucléus contre la membrane. l.ongtemps cetle masse reste immobile, tandis que d’autres organes se développent autour d’elle. sauf que les glo- 
bules se divisent it sit prortie posterlente: puis elte se divise en lobes, el la masse contenue dans les cellules tombe en lragnents prenant une teinte jaune-brune. Cel organe ne peut être que le foic dans l'opinion de l'auleur. Unn groupe de cellules de chafue côté de l'ossophage donne naissance aux glandes salivaires. (ie que Gegenbaur a olsservé de la formation du tube digestil chez Clausilia et Helix coneorde avee les données qui viemnent d'ètre rapportées.

C. Vogu ( cxxix) al vu, chez les famenses larves qu'il a observées à Nice, larres (fui ont élé ballottées d’un gente à l'autre et qui ne sont. après tout, que des larres de lyaléacées, le tube digestif composé d'un resophage, d'un estomac et d'un intestin. Deux organes dépendent de l'estomac, à saroir: un foie "cellulaire " et un recum large. Je ne réussis pas à reconnaître nettement ces détails sur les figures, qui ont été publićes plus tard (cvisi).

Koren et Danielssen (Lxxx) croient atroir ru l'estomar se lormer, chez Buccinum et Purpura, par l'exsudation de l'un des vitellus que renferme l'embryon: cette observation est évidemment enlarhée de la même errur que leurs idées sur la segmentation. Le foie brunatre se différencie sur les còtés de l'estomac, les glandes salivaires naissent de deux amas de cellules silués sur les côtés de l'usophage. Cess deux amas s'allongent el entrent en communication arec le tube digestif; du pigment jaune se montre dans leur's parois. Plus tard, elles se sondent en une seule glande munie de deux canaux excréteurs. Dans un travail postérieur (ctx), les mêmes auteurs soutiennent, contrairement à l'opinion de Carpenter (cus), que l'(esophage ne se forme que trèstard et entre en communication avec un estomac préformé. Le foie se formerail par la soudure de trois corps opaques qui se montrent sur les còtés de l'estomac.

Chez Eutoconcha, J. Miiller (Lxxus) déerit l'aesophage, puis l'estomac. dont la partic postérieurese perd an milien d'un amas de collules. et rela rhe\% des larves très-arancées. Cet élat ludimentaire de la partie postérieure du tube intestinal, décrit par un anatomiste aussi soigncus, mérile d’ìtre nolé.

Le mime auteur décrit, che\% des latres de ('reseis (cxxin!), un long crecun qui part de l'estomac et atleint le fond de la roquille, cercum qu'il liut se gatrder de eonfondre aree le fioie; ee dernier apparait. plus tard, comme un ciremu de l'estomtate el se divive ensuite en follicules.

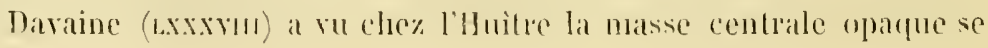


différencier en une partie claire qui domne naissance à l'eslomac et l'intestin, et me partie foncée gui donne naissance an foie.

Huxley (xci) a vu chez des jemnes de Creseis deux diverticules parlant de l'estomar; il parle de leur texture glandulaire et les compare aux direrticules ramifiés de l'intestin des Eolidiens. Il est probable, ainsi que le fait observer J. Miiller, que l'un de ces diverticules est le foie naissanf, l’autre le sac allongé, yu’il a drécrit précédemment (Lxxilli).

Lenckart (xсn) at r'u, chez les Firoloüdes, l'amas des cellules centrales se rleuser, et cette cavité entrer en communicalion arec les deux enfoncements opposés, la buuche et l'anus. D’après nes observations sur Pterotrachora, ce prélendu anus ne pent guère ètre autre chose que l'enfoncement roquillier.

0. Schmidt (xcv) roit, dans l’intérienr de l'embryon de r'yclas calyculata, une sphère rellulaire creuse, ciliće intérieurement, dont les parois se dissondraient pour ne laisser (qu'une vésicule qui disparâ̂Irait à son tour. An-dessus et en arrière de la carité centrale se formeraient deux rondensations de tissu cellulaire qui deriendraient le foic.

Gegenbanr (cı) remarque avé justesse que chez les Pléropodes la masse intérieure, composée de grosses rellules nutritives, se différenrie de manière à former le tractus intestinal. Le cul-rle-sac postérieur (sac nutritif), vn par Vogt, a échappé à son alteulion. Chez Tiedemannia, cet observalour ronsciencieux a remarqué que les grosses cellules centrales ne commencaient à montrer leur tendance à conslituer le tube digestif qu'apròs lapparition des rils vibratiles. Che\% Pterotrachacu, il a observé que la butuhe ot liusophage se forment part enfoncement de l'ertoderme, qui pousse à la rencontre des cellules centrales. Il conteste arec raison que l'anus apparaisse, comme l'a cru Leuckart, comme un enfoncement de la couche externe au pôle aboral.

Leydig (cu) montre que les jeunes embryons de Cyclas cornea ont une masse centrale opaque, à la reneontre de laquelle s'enfonce une fossette qui se tapisse de cils, tindis que l'anas central lui-mème se ereuse pour derenir l'estomar. Le foie se forme clairement par éraginations de la paroi de l'estomac: on voit d’abord sur les côtés de ce dernier oryane denx cacums ciliés intérieurement qui poussent et se divisent, en sorte quil y a me dent-donzaine de ces recums de rhaque côté de l'estomac, rer's la lin de la rie embryomnaire. Les

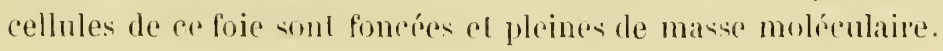


Carpenter (cvi) montro que linsophage se forme de bonne leure chez les embryons de Prupura, et mène simplement à une masse cen. trale, tomposéc de vitellus avalés. Ce n’est que quand l'embryon est ainsi rempli d'ceul's stériles, ([n’il commence à se dérelopper. Plus tard (cxvi), le mème auteur confirme à noureau ses données en opposilion à celles de Koren et Danielssen (Ixxx).

De Lacaze-Duthiers (cxir) nontre que chez Dentalium l'embryon possède mu amas interne trìs-oparque, d'où sortent les organes de la digestion. La bouche se montie, et l'anus, qui n’est d'abord qu'un jetit tubercule de la face ventrale, apparaît derrière le talon du pied. Le tubercule se creuse el correspond à un tube cilié, l'intestin. Lat masse centrale, située vers le còté dorsal, se compose de grosses granulations; elle se creuse par érosion et renferme un liquicle où llotlent des globules. Les granulations des parois prennent l'aspect de qunttes de graisse. Cet observateur habile ef circonspect a ru cusuite cette cavité entrer en communication arec celles de la bouche el de l'anns; il dome à cetle partic centrale du tube digestif le nom trisbien choisi de blasteme gastro-hépatique. Celte ravité se gamil de cils rihratiles. Elle représente l'estomac et le foze encore confondus. Du vingtrinquième au lrente-cinquième jour naissent, sur les côtés de cette poche, des lobes perpendiculaires à la paroi, lobes jannàtres el épais, gui se divisent en lobes secondaires, se creusent, et constituent ainsi le foie. La traince de blastème, qui donne naissance ì l’intestin, est ume dépendance scrontaire de la masse centrale. La poche linguale se forme comme poche de l'nsophage ol les rarfilages se différencient ì ses côlés.

Claparède (cxiul) trouve aussi chuz Neritina l'amas de substance mutritive au centre de l'embryon et montre que l'wesophage et le lube digestif se déreloppent iei plus tôt que chez d’autres larves. La larve s'en sert immécliatement pour lécher les autres vitellus, yu’elle absorbe ainsi pelit à petil. L'mesophage est une invagination de l'cetoderme; l'amas central se sépare en deux unasses sphériques, dont l'une correspond à l'estomac el l'autre au foic. Ces lleux organes se composent d'abord de grosses cellules réfringentes, qui se transforment directementen éléments épithéliaux dans un cas, et glandulaires dans l'autre. Ces dernières ne portent pas de cils vibratiles. Tes cartilages de la langue se différencient sur les côtós de l'resophage.

J. Miiller (cxix) revient sur ses données précédentes relatives au foie des Ptéropodes orthoconcques of de C'messis an particulier. pour les 
confirmer et.les acrentuer. Le diverticule qui pend au-dessous de l'estomac n'est pas le foie; il acquicrt sa plus grande importance pendant la vie larraire el diminue plus tard; ses parois ont un aspect glandulaire; son conteu est parfois jaune, parfois incolore. Ses couches épithéliale et musculaire sont en continuilé avec les mêmes. couches de l'estonac. Il a des contractions péristalliques. Le foie naît sous forme de pelits follicules, qui poussent su le canal efférent du diverticule, à l'endroit où il débouche dans le bas de l'estomac, et finit par former une grappe, derrière laquelle pend le diverticule. Cies deux organes ont été parfaitement décrits et figurés par Eydour et Souleyel (Voyaye de la Bomite) chez diverses espèces des genres Hyalcea et Cleorlora.

Schneider (cxi) déerit brièvement le tube digestif des larves de Phyllirhoë d'une manière conforme aux donnćes précédentes sur les larres de Gymnobranches.

De Lacaze-Duthiers (cxim), dans son beau travail sul le Vermet, montre que la bouche se forme au point où la masse périphérique vient en dernier lieu se rejoindre par-dessus la rouche centrale. La bouche, qui est bientôt entourée par les rudiments du voile et du pier, paraît n’être que le résultal du recouvrement incomplet de lis masse centrale par la couche périphérique; elle paraîl comme un point noir, qui se trouve bientôt au fond d'un infundibulum fortement cilié. La cavité digestive se forme par creusement dans l'intérieur de la masse centrale et entre en communicalion arec l'usophage. Lanus sourre à droite. Lo foie se forme aux dépens de la masse vitclline centrale à peu près entière, sauf la cavité centrale qui rlnnne naissance à l'esiomac.

Krohn-(CXX) s'est attaché surtout à étudier la métamorphose des larres de Péropodes et d’Hétéropodes el à élablir les transitions entre ces larres pélagirunes et les animaux adultes. Le consciencieux observaleur décrii cinq formes différentes de larves de Ptéropoles gymmosomes. Chez les deux premières, qui sont peut-être des lanes the Pnemmodermon, le tube digestif, peu avant la métamorphose, est très. coloré. l'auus siluć à droite; l'estomac porle à sa face interne une plarque solide garnie de petites dents. Le foie se montre sous forme d'm cacum brun qui se contracte ct se dilate alternatirement, et répond au crocum des larres de C’rseis ; il est très-allongé chez l'une de ces deux espèces. Après la métamorphose, h luhe digestif se ra-

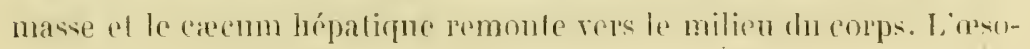


phage portr sur ses côlés deux sacs à crochets, qui ne. sont que des élargissements de ce tube. Chez la troisième forme de larres, t'estomac porte aussi une plaque dentaire; mais le foie constitue me masse ovale, siluée surtout au côté gauche de l’estomac. Chez la quatrième al cinquiome lorme, le foie vecupe la même position el présente les mèmes contours. La cinquième larve porte une plaque dentaire dans son estomac, plaque qui persiste encore après la mélamorphose ; sur

- les còtés do la bouche se trourent, après la transformation, des bras munis de papilles, qui portent des pointes au lien de crochets. Toutes ces larves ont l'osophage à gauche et l'intestin à droite de l'estomac; le loie se présente sous deux formes différentes. Che\% les larres de rymbulir, pen avant leur mélamorphose, l’anus se trouve à droite; le loie est incolore et divisé en deux lobes, qui s’étendent jusqu’an fond de la corquille. Che'z les jeunes, le toie est brun el orcupe la nème position que chez l'adulte. Les larres do Tiedemmmin ont un foic brunverdàtre, coloration qui provicnt des globes huileux rert-clair qu’il renferme, el dui se retrouvent chez ladulte. Le butche des Cymbuliacées a été décrite, aree une parfaite exactitude, par Eydoux el Souleyel; mais, ce point n’élant pas d'un intérèt génćrłal, il n’est pas nécessaire d'analysej les données qui s̀y rapportent.

Chez les Firolacies, le sagace observiteur rapporte que l'asophage est large el se tramslorme, pendant la métamorphose, en majeure partie en estomac. L'estomac larvaire a son pylore en bas à gauche ; l'inlestin remonte du côté droil. Le rudiment du foie se compose de deux carums contractiles, chassant leur contenu dans l'estonac, qui le leur renvoie. L'un de res racums occupe le fond de la coquille el débouche dans l'estomac du côté droit el à sa partie inléricure; l'autre se trouve entre l'estomac et l'intestin, et débouche probablement dans l'estomac, près de la naissance le ce dernier. Chez l'adulte, le foie n'a qu'un senl canal, s'ourrant dans un élargissenent intestinal, qui doil répondre à l'estomac larvaire. Chez Firoloüdes, l'estonrac de la larve est muni d'une plaque dentaire, qui ne s'est pas retrouvée chez Pterntrachea. Chez les jemes larves d'Atlanta, la disposilion du lube digestif paraît ressembler à ce ıui s'observe chez les Firolacées.

Keferstein et Ehler's (cxivis) ont vu chez des embryons d'Aotis un enfoncement à chacun des deux pôles opposés; plus tard, ils ont ru un enfoncement en $\mathrm{T}$, à savoir la bouche, pousser à la rencontue de la masse centrale, ol une antre onrerture, celle de l'auns, se former non pas au pòlo opposé à la bouche, mais sum le côté. Le lube intes- 
tinal se différencie ensuite et se garnit de cils; l'estomac est flanqué de deux lobes hépatiques, dont l'un, celui de gauche, va en grandissant, tandis que l'autre diminue.

Lereboullet (cxxvin) montre que, chez l'embryon de Lymncus, la cavité centrale reste en commmication avec l'ouverture d'invagination, qui devient la bouche. Cette cavité digestive est entourée de cellules allongées et, plus à l'extérieur, de grosses cellules rondes, dans lesquelles les sphères vitellines se forment par génération endogène. Ces sphères sc groupent en deux lobes, et constituent ce que l'on a improprement appelé le foie; improprement, car ses éléments doivent subir encore une transformation arant de derenir des éléments hépatiques, et jouent, en attendant, le rôle de vitellus de nutrition. Au pôle aboral apparaît une dépression, qui prend un grand développement, et forme un tube: le tube rectal. Ce tube est ouvert à son extrémité interne, mais la cavité digestive est fermée en arrière et ne communique pas avec lui. L'anus, c'est-à-dire l'ouverture externe de ce tube, est le point de départ d'un bourrelet circulaire, qui est Iui-même le point de départ du manteau. Bientôt après, l'anus se retrouve, non plus au centre, mais en dehors du bourrelet à droite ; et pourtant le bourrelet est toujours circulaire et entier. Cette fois-ci, l'anus communique bien par un intestin avec la cavité digestive. Il est repoussé de plus en plus en arant, à mesure que le bourrelet s'avance vers la tête. Il y a dans cette description des contradictions évidentes dont l'auteur ne paraît pas s'ètre aperçu; l'ouverture en question ne peut sauter du milieu du bourrelet à son côté externe sans l'entamer. Ganine (Xxxix) a fait voir que ce soi-disant tube rectal n'est autre chose que l'origine du manteau.

La bouche forme un tube saillant, l'œsophage et le rectum sont courts, l'estomac, un sac déprimé dont les parois se différencieront en une couche muqueuse et une couche musculaire. L'intestin se courbe, puis s'allonge et forme des anses. Les résicules vitellines sont toujours arrangées en deux lobes, qui forment en avant un angle ouvert; elles sont accolées extériemrement à l'épithèle du tube digestif, et s'arrangent en petites pyramides. Ces résicules changent de propriétés chi miques et domnent, sans doute par fractionnement, naissance anx résicules nourelles, bien plus petites, qui deviendront les cellules biliaires. C'est au sommet du tortillon que les vésicules vitellines subsistent le plus longtemps. Peu avant l'éclosion, lon voit sur les côtés de l'intestin, à sa naissance, deux petits utricules inégaux, yuu s'ou- 
rrent dans une partie élargie de l'intestin, tout près de l'estomac. Ces utricules se retrouvent chez des jemnes de deux mois; l'auteur ne sait s'il doil les considérer comme des appendices pyloriques ou comme l'njigine des canaux cholédoques. Dans ce caś, il faudrait qu’ils s'allongeassent à la rencontre de la glande hépatique. On ne peut s'empècher, en lisant cette description, de songer à ce que j'ai ru chez les Ptéropodes. Quant à la glande biliaire, elle se composerait d'amas de petites cellules et de petites vésicules, qui sont accolées extérieurement à l'inlestin, au rectum et aux appendices pyloriques. Chez des jemnes de quinze jours, toute la glande hépatique se composerait de cellules à mucléus et nucléole, d'un beau jaunc-lorun, et de quelques vésicules graisseuses.

Semper (cxxxu) rapporte que chez Ampullaria polita, l'entoderme, après s'être détaché de l'ectoderme, est encore relié à celui-ci par un amas de cellules, qui formera l'intestin. Les cellules de la masse interue grossissent et sont divisées en deux lobes, par une ligne claire qui est sur la continuation de l'œsophage. Cette ligne claire est un canal qui deviendra l'estomac et l'intestin, tandis que les lobes latéraux donnent naissance aux lobes du foie, dont l'un s'étend en arrière dans la coquille, et l'autre en avant jusque près de la bouche.

Selenka (cxxxv) rapporte que chez T'ergipes claviger le feuillet interne donne naissance au tube intestinal et au foie, ciliés dès qu'ils se sont ereusés. Les cils renvoient les particules du foie dans l'estomac et vice versü. Le mème auteur, après avoir décrit la formation des deux feuillets par épibolie chez Purpura, montre que l'anus se forme par une petite invagination de la couche externe, croissant à la rencontre de la couche interne, avec laquelle elle se soude.

Salensky (cxxxrul) décrit chez Calyptrcea, après la formation des deux feuillets, l'apparition d'un amas ectodermique, au milieu duquel se forme $u$ enfoncement primitif. Du fond de cette fossette part un tube, qui s'allonge en arrière, puis se détourne à droite. Les sphérules nutritives centrales sont dépourvues de nucléus; elles se divisent. Un bourrelet de cellules se forme au milieu de l'embryon, venant de la bouche et se courhant à droite. Ce sera l'origine du tube digestif. Je n’ai pu réussic à bien comprendre quels sont les éléments. les fenillets dont provient ce bourrelet central, dans l'opinion de l'auteur.

Ganine (cxxix) litpporte que, chez C'yclas, le tube digestif se forme par creusement de ce feüillet interne, que nous avons vu provenir d'un dédoublement local de l'ectoderme primitif. Il domne naissance à 
l'osophage, à l'estomac et à l’intestin; il perce, à l'extérieur, d'abord à l'extrémité lsuccale, puis à l'extrémité anale. Chez Lymnæus, Planorbis, Physa, il se forme, au côté ventral, un enfoncement qu'entourent, comme chez Vermetus et Calyptrxa, les rudiments du voile et du pied. La bouche se forme au fond de cet enfoncement. Le leuillet interne, d'abord infundibuliforme, devient sphérique, et communique avec l'extérieur, d'ahord par la bouche, puis par l'enfoncement ectodermique de l'anus. Le foie se forme ici, de même que chez C'yclas, assez tard comme processus creux, qui naît de la paroi du lube digestif entre l'eslomac et l'intestin, et cela, sans participation aucune des éléments nutritifs.

Stepanoff (cxxxvıi) fait provenir, chez Calyptræa, l'œesophage d'un enfoncement de l'ectoderme, le tube digestif d'une différenciation de l'amas nutritif; le foie est à l'origine un diverticule du sac stomacal, contenant dans ses parois du pigment et des gouttes de graisse.

E. Ray Lankester (CxL) a vu chez Aplysia les cellules de l'amas formatif, qui résultent de la division des deux cellules formatives, s'introduire entre les cellules centrales et s'augmenter aux dépens de ces dernières. La prolifération n'a lieı que depuis le pôle formatif. Le foie croît probablement aux dépens des cellules centrales, mais ne descend pas d'elles, car elles se comportent d'une manière entièrement passive. T.'ossophage se forme par invagination de l'ectoderme.

Langerhans (cxLI) a remarqué le premier, chez l'embryon tout formé d'Acera bull., de Doris et d'AEolis, deux cellules cctodermiques qui se différencient de très-bonne heure des cellules environnantes, et font saillie à la surface. Ces deux cellules sont munies d'abord d'un nucléus, qui redisparaît. Ces cellules prennent naissance, chez Acera, sur les bords de l'épaississement de l'ectoderme, qui marque le point où se trouvaient les deux cellules formatives; chez Doris, elles se trouvent sur le prolongement de l'axe longitudinal de la fente, résultant de la fermeture de l'ouverture d'invagination primitive. C'est entre ces deux cellules que s'ouvrira l'anus. Le tube digestif se forme, chez Acera, par le creusement de ce cordon cellulaire, qui provient de deux proliférations de l'ectoderme; chez les Gymnobranches, par différenciation rle la cavité d'invagination.

Les jolis dessins de Salensky (cuni) sur le déreloppement de l'Huître montrent que l'entoderme donne najsance à tout le tube digestit, sauf la bouche el probablement l'opsophage, qui proviennent d'un 
enfoncement de l'ectoderme. lls sont d'accord en cela arec les beaux dessins de Lovén.

Si nous cherchons à mettre quelque ordre dans toutes ces données éparses, nous sommes amenés, arant tout, à distinguer les mèmes denx types que nous arons rencontrés dans la formation des feuillets. Dans le premier type, celui où l'entoderme nutritif donne naissance an tube digestif, nous voyons l'estomae, l'intestin et ses appendices embryonnaires dériver directement des cellules qui renferment la provision de nourriture de l'embryon, quel que soit, du reste, le pro cédé par lequel ces éléments nutritifs ont été englobés par l'ectoderme. La seule partie du tube digestil' qui provienne de l'ectoderme est la bouche avec l'œsophage. Chez divers Lamellibranches et Céphalophores, les parties latérales de l'entoderme forment deux sacs qui restent en communication arec l'estomac ou le sommet de l'intestin. La plupart des auteurs admettent que ces sacs, qui renferment dans leurs parois la presque totalité de la substance nutritice, sont l'origine dn foie. Il n'est pas douteux qu'il n'en soit ainsi dans certains eas ; mais ce fail est-il aussi général qu'on l'a cru? Ce point demanderait à être approfondi. Une-difficulté pratique a beaucoup contribué, sans doute, à empècher son éclaircissement, à savoir : l'impossibilité d'élever complétement les larves de la plupart de ces Mollusques. Des r'echerches comparatives, spécialement dirigées dans le but de mettre au net ce développement du foie, seront nécessaires, et les résultats obtenus chez Dentalium, Cyclas (daprès Leydig) et les Ptéropodes fourniront déjà quclques points de repère. Que ces deux lobes lassent ou non partie in tégrante du foie de l'adulte, il parait admissible que, dans beaucoup de cas, le foie ne se forme pas en entier à leurs dépens, et qư ils jouent, pendant une partie du déreloppement, un ròle bien différent. Dans le second type, celui des Aplysiens, de quelques Pulmonés et de C'yclas (d'après Ganine), celui où il se déreloppe un entoderme de nutrition et un entoderme de formation, ce dernier donne seul naissance au tube digestif, tandis que le premier se résorbe purement et simplement. Dans ce cas, il paraît, d'après Ganine, que le foie n'est qu'une éragination de la paroi du lube digestif, sans participation aucune, directe ou indirecte, des éléments uutritifs. 
CILS VIBRATILES ET VOILE.

La rotation des embryons de Mollusques est un des phénomènes qui ont attiré le plus l'attention des premier's embryogénistes. Les causes de la rotation ont donné lieu à nombre de discussions, jusqu'à ce que les moyens optiques fussent devenus assez parfaits pour permettre de distinguer les cils très-fins, dans beaucoup de cas, qui en sont la cause. Aussi l'histoire de la découverte des cils du Lymnée, par exemple, n'aurait-elle que peu d'intérêt, l'habileté de l'observateur joulant en ceci un rôle bien moins important que celle de l'opticien. Chez les larves qui ont des cils plus gros et plus faciles à voir, ces cils moteurs ont été découverts et compris de très-bonne heure. C'est à tort que l'on attribue leur découverte à Grant (xvin); ainsi que l'a fait observer Salensky, ces cils ont été fort bien vus et compris par Forskal (ri), qui dit dans sa description des larves de Janthina : Nam ad aperturam duo vela transversa, subrotunda, pilis tremulis ciliata, quibus pulli hi remigabant...Bommé (v) avait déjà vu précédemment dans une ponte de Doris se former une quantité de "Rotifères". Carus (xi, xv, Xxi) et plus tard Jacquemin (xxiv et xL), Laurent (xxviu) et d'autres, nayant pas réussi à roir les cils fort petits des Mollusques d'eau douce, ont cherché à expliquer la rotation par des théories plus ou moins ingénieuses. Lerehoullet (cxxyni) mème n’a pas réussi à roil les cils de la larve de Lymmous, ce qui ne fait pas l'éloge de son microscope. Grant (xviI), Lund (xxiI), Sar's (xxiv) et tous les auteur's subséquents ont vu et compris les cils et les disques moteurs des Céphalophores marins. Van Beneden et Windischmann (XLIV) ont ru les cils très-fins qui conrrent la résicule céphalique et les tentacules de Limax. Le voile arec les cils moteurs a été signalé chez les Lamellibranches par Holböll (L) pour Modiolus.

Alder et Hancock (LI) décrivent la forme des disques moteurs arrondis des Gymmobranches et la position horizontale qu'ils prennent pendant la natation, position qui derient rerticale lorsque l'animal se rétracte dans sa coquille. Von Nordmann (LII) montre que ehez Tergipes deux manelons, naissant sur les còtés de la bouche. sont l'origine du voile; les cils y apparaissent et grandissent rite et le voile lui-mòme frend une forme de $S$. Chaque cellule du bourrelet ne porte qu un eil moteur. Si elle vient à se détacher, le cil continue à battre pendant des heures. Après la métamorphose. Ie voile se réduit à deux petits 
mamelons ciliés, situés sur le front, et puis tombe. Ciet auteur, ainsi que Sars (LVI1), remarque que chez les Gymnobranches les cils moteurs sont soumis à la volonté de la larve. C. Vogt (LxiI) a remarqué che\% Acteon les muscles qui se rendent au bord du voile, et qu'il nomme simplement des fibres; il décrit les cellules du bourrelet moteur comme des bulbilles nucléées. Les disques moteurs ont la forme de deux demi-entonnoirs. Les cils moteurs sont de plus en plus soumis à la volonté, à mesure qu'ils se forment. Ce sont les premiers cils qui se montrent à la surface de l'embryon, au moment où il commence à prendre une forme triangulaire. Reid (LXIV) décrit la formation et les détails du voile de plusieurs Gymnobranches, sans rien ajouter à ce qu'en a dit C. Vogt. A. Karsch (Lxi1) a réussi à voir les cils qui cansent la rotation chez Lymnceus.

Lovén (Lxxl) rapporte que l'embryon de Modiolaria se couvre de petits cils et se met à tourner. Chez Cardium deux mamelons naissent d'un côté de la bouche (côté céphalique peut-être?), qui s'éloignent l'un de l'autre, se garnissent de cils et deviennent le voile. Au milieu de la surface supérieure el convexe de ect organe, se trouve un grand rirrhe unique, implanté sur un corpuscule rond.

Ghez Montacuta, les larves, au moment où elles naissent, ont un voile ovale, à bourrelet épais, muni de grands cils et de fibres ramifiées. qui partent principalement d'un point de la partie céphalique de la larve. Ces fibres sont munics par-ci par-là de corpuscules ronds ef seraient en partie des muscles et en partie des nerfs. Au milieu du roile se trouve le grand cirrhe, implanté sur un corpuscule cordiforme. Le célèbre naturaliste suédois pense que le voile ne tombe pas complétement, mais se transforme pour donner naissance aux tentacules buccaux. Leuckart (Anat.-phys. Ubers. des Thierreichs, p. 675̈) montre que l'Anodonta intermedia possède ì l'état larvaire $u$ voile bien reconnaissable.

De Quatrefages (Lxxmi) a vu chez T'eredo les cils apparaitre comme de petits mamelons transparents, qui s'allongent ensuite. Le roile grandit et forme deux lobes; il a un bourrelet, muni d'un seul rang de grands cils. Des muscles, déjà rus par Lorén, se rendent de la charnière au bord du voile.

Nax Schultze (Lxxu) a ru chez Tergipes le voile se ratatiner lor's de la métamorphose et le bourrelet se détacher par morceaux. jusqu à deux petits amas irréguliers, qui tombent à leur tour.

Leydig (Lxxir) montre rque le voile naît, chez Paludina, romme denx 
mamelons sur les côtés de l'enfoncement buccal, mamelons qui se garnissent de cils plus longs que ceux qui courrent le reste de l'animal; il se présente plus tard sous forme de simple ligne ciliée et disparaît complétement, sans avoir jamais acquis un grand développement. La peau se courre alors de petites papilies, qui portent des touffes de cils fins.

Gegenbaur (Lxxvu) a vu l'embryon de Limax agrestis se couvrir tout entier de cils. Koren et Danielssen (Lxxx) rapportent que chez Buccinum et Purpura, le bourrelet voilier porte, outre la rangée de cils moteurs, une quantité de petits cils fins; le voile tombe sans laisser d'autre trace qu'une ligne transversale derrière les tentacules. Chez Entoconcha, le voile, d'après J. Müiller (Lxuxır), se réduit à un lobe porlant de grands cirrhes raides. G. Vogt (Lxix) montre que le roile des Gastéropodes pulmonés se réduit graduellement chez les genres d'eau douce, pour arriver à être nul chez les Pulmonés terrestres.

J. Mïller (LxxxıI et xcıI) décrit les larves de Ptéropodes gymnosomes, déjà vues par Eschscholtz (xrv) et par Busch (Lxxris), qui n'avaient pas reconnu leur véritable nature; ces larves portent trois couronnes de cils, l'une près de l'extrémité antérieure, derrière la bouche et les ventouses, l'autre au milieu du corps, derrière le pied et l'anus, et la troisième près de l'extrémilé postérieure. Chez les larves de Cleodora aciculata le voile est bilobé et chaque lobe est divisé à son tour en deux, par une échancrure de son hord externe.

Chez Ostræa, Davaine (Lxxxvir) a vu le voile se montrer d'abord sous forme de deux cellules ciliées, très-écartées l'une de l'autre; puis la couronne de gros cils se développe et le voile prend la forme d'un entonnoir portant, à la ligne médiane, une rangée de grands cirrhes qui aboutit à la bouche. Ge voile se détacherait et tomberait sans laisser de traces.

De Lacaze-Duthiers montre (xar) que ces observations ne peuvent se rapporter qu’à des Iarres malades, et que le disque moteur va en augmentant pendant plus de quarante jours, et entre en rapport de plus en plus immédiat avec la bouche.

Chez Cyclas cornea, Leydig (cri) a indiqué la présence, à l'extrémité céphalique de l'embryon, de grands cils qui constituent un voile rudimentaire.

D'après Leuckart (xcı), l'embryon de Firolüdes se courre en entier d'une toison uniforme de cils, et se met à tourner. II prend une forme triangulaire, les angles supérieurs du triangle devenant le bor'd du roile. 
Gegenbaur (cr) a ru l'embryon de sa Cleodor'a pyramidata (qui est le mème que la C\%. Ianceolata, Lesueur) se couvrir en entier de petits cils avant l'apparition du voile. Il m'a été impossible de retrourer ee starle. Les observations de ee savant distingué sur le genre Hyalcę concordent mieux avec les miennes. Iei se forme, dès l'abord, seulement une couronne de grands cils, rapprochée du bout eéphalique de l'embryon; le bourrelet qui porte ces cils s'élargit, prend une forme de 8 , et dans l'intérieur se montrent des fibres radiaires et ramifiées. Dans l'échancrure antérieure du voile, se trouve ehez los Ptéropodes, comme chez tous les Mollusques à voile, l'ourerture de la bouche. Le même auteur décrit des larves à voile horizontal trèsgrand, et muni de fibres ramifiées très-développées, qu’il croit pouvoir rapporter au genre ('reseis, mais qui ne sont probablement que des lanves de Gymnosomes. Dans l'intérieur du voile se trouvent de très-grandes cellules que Krohn a revues plus tard. Iei le bourrelet voilier est creusé en gouttière à son còté extérieur ; le bord supérieur de la gouttière portant les grands cils moleurs, le bord inférieur, de petits eils très-fins. Krolın ( $\mathrm{cxxv}$ ) remarque que celte double rangée de cils se trouve chez beaucoup de Gastéropodes.

Une autre larve, qui est bien celle de Creseis, a un voile composé de deux lobes très-échanerés à leur côté externe, comme le Cirrhopteron de Sar's.

Ghez Tiedemannia, il se forme une toison de cils qui eouvre tout l'embryon; le bourrelet du roile se montre ensuite au tiers antérieur de la larve et prend la forme du voile des Gastéropodes, tandis que le reste de la toison ciliaire disparaît.

Gegenbaur pensait que le voile des Ptéropodes thécosomes n'était pas un organe purement larvaire, et croyait le retrouver dans les lignes vibratiles de la base des nageoires de ces animaux. J. Mïller (cxx) a montré qu'il ne pouvait en être ainsi, puisque ces lignes, qui ne sont en réalité que le bord d'un champ tont couvert de cils, se trouvent à la face ventrale et non à la face dorsale des nageoires. Le voile est done, ici comme ailleurs, un organe purement larvaire. II a été observé par Lovén (Lxxi) ehe\% des larves de Spirialis.

Chez les Gymnosomes, Gegenbaur (ci) décrit fort bien les trois anneaux ciliaires de la seconde forme larraire; chez une larve, qui possédait encore un roile, il n’a réussi à distinguer que les deux rouromnes postérieures, ee qui l'induit à croire que la couronne antérieure de ces lartes répond au bourrelet roilicr. 
Krohn (cx et cxxy) et J. Nïller (cxx) ont montré que c'était une erreur, et que la couronne eiliaire antérieure apparaît au-dessous dı voile, et avant la ehute de ce dernier. Le roile des larves de Gymnosomes atteint souvent, d'après Krohn, des dimensions telles, qüil ne peut se rentrer dans la roquille; il se compose de deux lohes entier. à leur bord externe. Les trois couronnes de eils de la seconde période larvaire se montrent au moment on le voile et la eoquille tombent. Ces eouronnes ne tombent qu'au moment oì l'animal atteint l'état adulte, la couronne antérieure disparaissant la première, puis celle du milieu, et enfin la couronne postérieure.

Chez les Gymbuliacées, le voile des larves prêtes à se métamorphoser est divisé en deux lobes très-échanerés à leur bord externe; il tombe eomplétement lors de la métamorphose.

Gegenbaur (cI) eroit avoir trouvé, chez Pterotrachcea, un stade où l'embryon serait couvert d'un duret uniforme de eils, qui se limiteraient ensuile au bourrelet du roile. Ce bourrelet est continu chez les Ptéropodes et les Hétéropodes; il peut s'infléchir par places, mais sans s’interrompre. Chez Pterotrachcea les deux lobes du roile s'échancrent à leur bord externe, au point de former deux lanières dirigées en arrière et deux lanières en avant. Chez Atlanta, le voile présente deux échancrures de chaque côté, formant ainsi quatres lobes dirigés en arrière et deux lohes dirigés en avant. Le bourrelet voilier est ereusé en gouge, et porte de grands eils au bord supérieur, de pelits cils au bord inférieur de cette gouge.

Krohn (cxxv) confirme les données de Gegenbaur sur la forme du voile de Pterotrachce et les étend à d'autres gemres de la famille des Firolacées. Le roile tombe lors de la métamorphose et la larve hâte sa chute en l'avalant! Chez Carinaria comme ehez Atlanta chaque lobe du voile se divise d'abord en deux lanières, l'une antérieure, l'autre postérieure, et eette dernière se divise à son tour en deux.

Maedonald (Cxxi) décrit diverses formes de larves pélagiques, qu’il prend d'abord pour des genres nouveaux; il arrive ensuite à reeonnaître qu’il ne s'agit que des larves de divers Cténobranehes. Ces larves conservent pendant très-longtemps un voile, composé de quatre lobes chez Ranella, Natica, Calyptrea, Cyprea, Murex et Buccinum, et de six lobes, comme ceux d’Atlanta, chez le genre Strombus. Déjà précédemment Krohn (Lxxxy, cv et cxviri) était arrivé à reeomnaitre que son Echimospria, avee un voile partagé en six lobes et un bourrelet creusé 
en gouttière portant deux rangées inégales de cils, n’élait que la larve d'une Marsenia.

Lovén (cv) montre que les larves de Chiton ont, comme les Lamellibranches, une couronne de cils près de l'extrémité céphalique, celte extrémité clle-même étant munie d'un pinceau de cirrhes peu mobiles. Ces organes moteurs de la larve tombent, sans avoir alcquis un plus grand développement.

Les recherches faites par de Lacaze-Duthiers sur te genre Dentalium (cxur) ont montré que les larves de ce Mollusque se couvrent d'abord de houppes de eils, qui s'arrangent ensuite de manière à former quatre zones ciliaires. L’extrémité antérieure est marquée d'une dépression, qui porte une houppe isolée. Les quatre zones se rapprochent ensuite les mes des autres, en se portant rers la partie céplialique, et se fondent en un seul bourrelet, qu'un sillon circulaire sépare du reste du corps ; ils constituent de la sorte le disque moteur circulaire, qui disparaitra après avoir été recourert par le bord supérieur de la coquille.

Claparède (cxim) a vu l'embryon de Neritina fluv. couvert en entier de cils vibratils, qui le font tourner. Hlus tard cet excellent observateur a Irouvé les larves munies d'un roile de la forme ordinaire pour les Céphalophores. L'existence de ce voile avait été déjà signalée par Lindström (CIII). Le bouruelel roilier se compose d'après Claparède d'une seule rangée de cellules nucléées; les membranes qui relient ce bourrelet à la partie céphalique de la larve, sont un simple épithèle aplati. Dans l'épaisseul' du voile se trourent des cellules fusiformes ou ramifiées contractiles, munies d'un nucléus et d'un contenu fibrillaire.

Les cils du voile n'ont guère d'autre fonction, chez Neritina, que relle d'amener les particules nutritives jusqu'à la bouche. Cet organe se réduit ensuite à un petit hourrelet, situé à la base des tentacules, el finit par disparaitre entièrement.

Claparède fait observer que, tandis que l'embryon est tout couvert de cils, la larve n'a dé cils qu'au pied et, au voile et que chez l'adulte toutes les surfaces libres sont de nouveau couvertes d'une toison ciliaire.

De Lacaze-Duthier's (cxxıli) montre que l'origine du voile chez Vermetus se trouve dans deux petits mamelons qui, arec le rudiment du pied, forment un triangle autour de la bouche. Ces mamelons s'allongent, rout se rejoindre du còté dorsal et se couvrent de cils. Le vnile prend ensuite la forme ordinaire bilobée, se munit de fibres 
musculaires, puis redisparaît. Les cils moteurs, outre leur rôle d'organes locomoteur's, scrvent encore à amener à la bouche les particules nutritives.

D’après Krohn (cxxıv), le Gasteropteron, à l'état de larve, possède un voile composé de deux grands lobes ovales.

Schneider (cxx), Keferstein et Ehlers (cxxvil) el Langerhans (cxul) ont vu, chez Phyllirhoë, Lotis et Doris, les cils se montrer sur deux saillies latérales de la partie éphaliøue de I'embryon, ainsi que Vogt l'avait déjì observé chez Actcoon. Ces cils ne sont pas précédés de l'apparition d'une toison ciliaire générale.

Stuart (cxix) rapporte que, chez les Aplysiens, les cils moteurs naissent sur deux saillies voisines de l'extrémité céphalique; mais le reste de l'ectoderme se couvre d'un duvet ciliaire continu et très-délicat. Les grands cils moteurs sont des bandes plates composées chacune d'une rangée de fibres museulaires amincies à l'extrémité et accolées côte à côte. Chaque fibre aurait une texture qui rappelle celle des fibrilles des muscles striés des Vertébrés. Ce serait une série de corpuscules réfringents en forme de tambours ou de cônes tronqués et réunis par du protoplasme. Ces fibres seraicnt peut-être elles-mêmes composées de fibrilles longitudinales.

L'embryon d'Ampullaria polita n'a pas de voile d'après Semper (cxxxi1) et ne porte que de petites houppes de cils, qui se généralisent graduellement. Plus tard, cependant, la larve présente en avant du pied deux lignes de cils vibratiles particulièrement développés, lignes qui se rejoindraient au-dessous de la bouche et seraient pent-être l'homologue du voile. Vitrina (sp.?), Bulimus citrinus, Vagimulus luzonicus et Paludina costata sont également dépourvus de roile, même à l'état rudimentaire; en revanche, Melania est un genre rivipare dont les larves n'ont pas de vésicules contractiles, mais possèdent un voile très-développé.

Chez Terqipes, d’après Selenka (cxxxv), les premicrs cils qui apparaissent sont ceux du voile. Plus tard il se troure au-dessous du bourrelet moteur un bourrelet plus mince, qui porte de petits cils. Chez Purpura, les cils se montrent d'abord près de la bouche, dans une situation qui répond au roile, puis sur tout le corps, sauf en un point qui répond au dos de la larve.

Le voile de Calyptror, d'après Salensky (cxxxviı), se forme de la manière déjì décrite chez Vermetus (cxxi11). Mais, après l'apparition des bourrelets sur les côtés de la bouche, l'embryon tout entier se courre 
de cils funs. Lectoderme du chanp cireonserit par le voile se soulève et se remplit de liquide, formant une vésicule céphalique; la couche ectodermique elle-même se change en une substance homogène renfermanl de grands nucléi, très-épaisse, el reliée à l'entoderme par des fibres musenlaires. Le voile de Trochus varius se forme comme chez les Ptéropodes. Stepanoff (cxxxvis) al vu aussi les embryons de Calyptreen se courrir d'une toison de cils fins qui tombent bientôt. Le voile se courre plus tard d'une cuticule sur sa surface.

Ganine (cxxxix) fait voir que les saillies symétriques qui se montrent chez Lymuxurs an-dessus du pied et sur les côtés de la bouche, sont lorigine du voile. Ces saillies avaient été déjà fort bien rues et décrites par Lereboullet (cxxvus); senlement, les eils vibratiles ayant complétement échappé à l'attention de cet observateur, il n’a pu arriver à une notion claire sur la nature de ces bourrelets. Ganine a vu ces bourrelets s'élendre, che\% Lymmerus, Planorlis et Pliysa, et se garnil' de cils.

Les dessins de Salensky (cxum) relatifs à Ostrea edulis montrent que le voile affecte, ehez la jeme larve, la forme d'une simple couronne de cils.

Cet ensemble d'observations montre que l'embryon des Céphalophores et des Lamellibranches pent parfois se courrir d'une toison générale de cils, toison qui parait, dans la plupart des cas, tomber et clisparaître avant l'apparition du voile. Le cas de Dentalium, où la toison assez étendue de eils se resserre, pour former le voile au lieu de tomber, est digne d'attention. Le voile lui-mème peut apparaître comme simple couronne ciliaire sur la larve encore oroïde et ne crmmencer à faire saillie que plus tard ; ce cas le plus simple se présente che\% les Pléropodes, chez diver's Lamellibranches, che\% C'hiton, che\% Trochus, etc. Ou bien il pent se former d'abord deux saillies latérales un peu au-dessus du niveau de la bouche, saillies qui donnent à l'embryon une forme triangulaire; ces saillies se couvrent ensnite de cils et donnent naissance aux lobes du roile. Tel est le cas d'Acfrmen et de tous les Gymnobranches observés juscu'à ce jour. Ou bien entìn les saillies latérales penvent être. à leur origine, en contact même arec la bouche, comme cela s'observe ehez Vermetus, Calyptrcea. Lymmerus, ot aller en s'élendant cusuite. Ce n'est que l'ordre d'apparition des diverses partics du voile qui varie; les processus fondamentaux resient les mèmes.

Gnel que soil son mode de déreloppement, le roile prend, sauf dans 
les cas rares (Chiton) où il conserve la forme embryonnaire, une croissance à peu près identique. Il est plus simplement discoïde chez les Lamellibranches et les Solénoconques, bijurti chez les Céphalophores. Il doit sa mohilité à des fibres musculaires ramifiées, qui s'insèrent à son bourrelet. II porte souvent à son bord exterue, outre la rangée des cils móteurs, une rangée inférieure de petils cils, dont la présence n'a encore été constatée que dlans peu de cas, mais qui pourrait ètre plus génélale qu’il ne semble l'après les données des auteurs.

Chez certaines lormes pélagiques de Céphalophores, le roile acquier't un grand développement et forme quatre lobes (divers Cténobranches et les Firolacées) ou même six lohes (Atlantr, Carinaria, Strombus, Marsenia). Mais, qu’il soit peu ou qu’il soit très-développé, il n’en est pas moins, chez tous les Céphalophores, un organe purement larvaire et destiné à tomber entièrement lors de la métamorphose.

Chez les Lamellibranches, la question de saroir si le voile donne naissance aux teutacules huceaux n'est pas encore résolue.

Le roile subit une réduction graduelle chez les Mollusques d'eau donce et disparaît entièrement chez les Pulmonés terrestres.

Les couromnes ciliaires de la seconde période larraire des Gymnosomes n'ont aucun rapport avec le voile que res animaux possèdent pendant la première période larvaire.

Les observations sur la constitution intime des cils moteurs sont trop isolées pour qu’il soit permis d'en faire la base de généralisations.

\section{LE PIED ET SES APPENDICES.}

Il serait inutile d'insister sur les domnées anciemnes relatives à cel organe; le pied a été vu; cela va sans dire, par les plus anciens embryogénistes qui se sont occupés du développement des Mollusques. Mais, les renseignements qu'ils nous donnent, sur la formation et la différenciation de cet organe, n’étant pas de nature à jeter de la lumière sur son mode de formation et ses homologies, je puis, sans inconvénient, les pásser sous silence.

Laurent (xxxl) a vu le pied de Limax s'aceuser de fort bonne heure et lui donne le nom de queue. Sar's (xaxiv, xxxix et xhI1) el Lovén (xL et Luv) ont vu le pied en forme de languette horizontale des Gymnobranches et des Bulićes, muni d'un opercule à sa face inférieure. Vau Beneden (xLn) a ru un pied de même forme et operculé chez Aplysiu. 
Dumortier (xxiI), Jacquemin (xL) et van Beneden et Windischmann (xuv) ont ru, chez Lymnceus, Planorbis et Limax, le pied se différencier en même temps que le bouclier (que les deux premiers auteurs confondent avec la tête), sous forme de protubérance occupant tout un côté de l'embryon. Leydig (Lxiv) arrive aux mêmes résultats pour Paludina vivipara.

Von Nordmann (Lui) a vu le pied naîire chez Tergipes Eduardsii, comme protubérance situéc au-dessons de la bouche; il prend ensuite la forme de langnetle horizontale, se munit d'un opercule à sa face inférieure et d'une toison de cils à sa face supéricure, et présente, lorsqu'on le regarde de profil, un lobe pointu à la base, près du voile. Lors de la métamorphose, l'opercule tombe, et le pied se recourbe en arrière. C. Vogt (Lxil) décrit el représente surtout très-hien l'origine du pied comme une saillie élendue de la face ventrale de l'embryon. Cette saillie devient ensuile celle langutette aplatie, déjà décrite par d'autres, qui s’étrangle un peu ì la base, et qui est munie d'un opereule en dessous et de cils à la face supérieure; ces derniers cils servent à amener les particules nutritives à la bouche. La paroi du pied se sćpare en deux couches, le derme et l'épiderme; l'opercule résulierait d'une transformation directe d'une partie de cette couche superficielle. A l'intérieur se trouvent trois paires de boules opaques. Max Schultze (cxxir) a vu l'opercule tomber et le petit pied de la larve du Tergipes lacin. s'allonger en arrière el prendre rapidement un accroissement très-grand.

Les recherches de Lovén (Lxxi) et de Quatrefages (Lxxiı) ne font guère que constater la différenciation tardive du pied chez divers Lamellibranches. Ce pied est très-jetil, et compris dans l'espace restreint que laissent entre cux les orifices de la bouche et de l'anus.

O. Schmidt (Lxxyı) décrit comme l'origine du pied, chez Limax, un épaississement de la couche exlerne de toute la face ventrale de l'embryon. Cette "plaque rentrale " forme ensuite une saillie conique, qui se recourbe. Les trois tentacules naissent de sa partie antérieure. Gegenbaur (Lxxyu) montre pour Limax, Clansilia et Helix que la saillic du pied se recourbe en arrière. Les muscles du pied sont des cellules du derme qui s’allongent et se soudent, leur noyau étant repoussé vers l'cxtérieur, et leur contenu prenant un aspect fibrillaire.

Vogt (Lxix) décrit, chez ses larres de Pneumoderme, qui sont, en réalité, des larres de Hyaléacées, un pied en forme de languelle et dépourvu d'opercule; il montre que les bords te ce pied se séparent 
sous forme de lobes, qui prennent un grand accroissement vertical, et en conclut, arec une parfaite justesse, que l'opinion émise par Eydoux et Souleyet, d'après laquelle les nageoires des Ptéropodes ne seraient qu'une modification du pied, se trouve vérifiée par l'embryogénie. Ces données sont confirmées par J. Mïller (Lxxxin) pour Cleodorr acic., qui montre, en même temps, que le pied de ce Ptéropode est cilié, ainsi que la base de ses nageoires. Chez les larves de Pneumodermon, le bord des nageoires porte des cirrhes raides. Gegenbaur (ci) décrit le pied mince, allongé et couvert de rils, des larves de Creseis. Chez les larves de Gymnosomes, il déerit la forme du pied et des nageoires, avec lenr musculature et les cirrhes raides de leur bord postérieur, ainsi que l'appendice en forme de lanière, qui est implanté au côté inférieur de l'extrémité de cet organe. Chez quelques-unes de ces larres, la présence du pied a échappé à son obserration, ce qui l'amène à admettre que cet organe ne se formerait ici que tard. J. Nïller (cxx) insiste avee raison sur le fait que les nageoires ne sonf "iue des excroissances du pied. Krohn (cxxv) décrit diverses larves de Gymnosomes avec une lanière eiliée occupant la place dupied, lanière qui est repoussée en dessous, lorsque le pied caractéristique en forme de fer à cheval de l'adulte vient à se former. La lanière ne paraît plus ètre, dès lors, qu'un appendice du pied. Les nageoires naissent sous forme de petits mamelons sur les côtés du pied et en contact arec la base de cet organe. Chez les Cymbuliacées, le même auteur décrit le pied étalé, dont le lobe médian porte un opercule à sa face inférieure, lont les parties latérales s'élargissent pour former les nageoires. Le sommet du lobe médian présente une légère échancrure, au-dessous de laquelle naît un prolongement eylindrique; ce prolongement devient très-mobile et se courre de cirrhes raides; il acquiert un assez grand déreloppement, mais tombe en même temps que l'opercule, au moment de la métamorphose.

Le pied de la larve d'Entoconcha, décrite par J. Miiller (Lxxxi1), est courbé par le milieu, porte un opercule, et présente, au licu de courbure, une papille creuse, menant dans ume cavité ciliée, dont la signifieation n'est pas connue. Entre le pied et la bouche, se trouve une éminence ciliée, yui disparaît pour fairc place à un lobe élargi et cilić, rappelant eelui de Natien.

0. Sehmidt (xcv) a ru le pied de Cycles calyculata naître sums forme de protubérance étendue, puis former un prolongenient en arrière. Deux petits lobes, qui naissent dans l'angle anfóriemr entre le pied at le man- 
teau, sont peut-être les tentacules buccaux. Leydig (cus) a vu le pied s'accentuer chez Cyclas cornea, comme protubérance à pen près dialmétralement opposée à la bouche : il est couvert de cils fins. Chez Chitom, d'après Lorén (cv), le pied nccupe dès l'origine la majeure partie de la face ventrale.

De Lacaze-Duthiers (cxil) montre que che\% Dentalium le pied apparaît comme proéminence au-dessous de la bouche. Il s'allonge en avant, et présente bien vile trois lobes, dont un médian et denx latéraux; puis, s'allongeant encore, il devient cylindrique, et porte ses lobes seulement à son extrémité. Il est couvert de cils, au point de faire mouvoir la larve seulement à l'aide de son action ciliaire. L'auteur avait déjà fait précédemment la même observation chez les jeunes de Mytilus rdulis (crir). Les muscles du pied du Dentale paraissent se différencier en lieu el place.

Le pied porte chez Buccinum el Purpura, au dire de Sass, Koren et Danielssen (cix), d'abord un opercule en dessous ef puis deux petits lolses latéraux supérieurs, qui en font une véritable gontlière ciliće aboutissant à l'oresophage.

Le pied de Neritina fluv. a, d'après Claparède (cxin), la mềme lơrme en gouge que Buccinum el les mêmes lobes latéraux, entre sa base et l'entrée de la houche; cette goutlière ciliée sert à la manducation des ueufs stériles.

('hez Vermetus, d'après de Lacaze-1)uthiers (cxxm), le pied naît comme pelit mamelon au-clessous de la bouche, très-rapproché des deux mamelons qui domnent naissance an voile; il commence par laire saillie en arrière, puis s'isole sous forme de langnetle, el se recourbe vers le bas. L'opercule apparaîl sur celle de ses faces qui regarde le corps. A la racine du pied naîl un tubercule, qui s'échantre au milieu de son bord antérieur; il s'allonge jusqu’au uiveau des tentacules. Mais les deux lobes latéraux, peu accentués du reste, s'eflacent et celte partie du pied se sépare en deux lames superposées; la lame supérieure donnera probablement naissance aux lèves allongée's de l'adulte. Au fond de la fente se trouvent trois laches james, les rudiments, sams doute, de la glande pédieuse. De chaque côté du pied et an milieu de sa longneur, se ironve un mamelon, qui n'a yu'une existence passagère.

La larve du Gasteropteron Meclielii possède, d'après Krohn (cxxıv), comme pied un vaste et puissant lobe plat, à contours arrondis, qui se rabat au loin sur les côtés du corps. Son extrémité postérieure se 
termine en une languette ciliée. Quoique l'arrangement des muscles de ce lobe rappelle la nageoine de Tiedemanmia, l'on n'a jamais ru le jeune animal s'en servir pour la natation; le voile remplit soul cette fonction.

Gegenbaur (ci) a décrit le pied des larves d'Hétéropodes, pi ed par faitement pareil à celui des larves de Gymmobranches. Krohn (cxxv montre que, chez les Firolacées, le pied, un pen échancré au milieu de son bord externe, porte au-dessous de cette échancrure un prolongement plus ou moins coloré de brun, et qui deviendra l'appendice eaudal. En avant du pied et à sa base, croît un processus cylindrique et très-mobile, muni de fibres musculaires et de tissu conjonctif élastique; ce processus s'aplatit latéralemenit, s'étale el donne naissance à la nageoire on pied-nageoire caractéristique des Hétéropodes; son extrémité tombe, laissant une petite cicatrice. Le pied en languette lui-même forme l'extrémité de la queue de l'adulte, au dos de laquelle l'on trouve encore l'opercule chez des exemplaires jeunes. Chez les Atlantacées, la nageoire peut être d'abord vermiforme, ou bien elle peut prendre du coup la forme définitive. La pointe du porte-opercule est parfois étirée en une lanière ciliće; la queure se forme entre le pied-nageoire et le porte-opercule, qui en constitue la partie postérieure. Carinaria établit le passage entre les deux familles d'Hétéropodes dont il a été question.

Lereboullet (cxxvin) fait, arec raison, selon moi, remonter l'origine du pied chez Lymmexus à une différenciation de la majeure partie de la région ventrale de l'embryon. La couche externe le cette régiun se compose de très-petiles cellules, à nucléi brillants et à membranes peu apparentes. Ensuite le pied forme une saillie conique ou arrondie et se sépare plıs ou moins en deux lobes à sa partie antérieure. Dans son intérieur, l'on distingue des cellules embryonnaires. Le pied s'allonge ensuite en arrière, et les cellules de son intérieur deviennent fusiformes et finissent par former un feutré. Les lobes s'effacent en majeure partie; mais une ligne claire, qui marque en long le milieu du pied, reste comme témoin de leur existence passée.

Salensky (cxxхris) et Stepanoff (cxxxmu) décrivent l'origine du pied thez Calyptrcea, d'une manière conforme à ce que de Lalcaze-Duthiers at vu chez Vermetus. La bouche commence par une fossette primitive, entourée d'unc saillie en forme de coeur de carte, qui se divise en trois mamelons, dont le plus inférieur domnera naissance atu pied. Ce dernier mamelon s’accentue et s'étend en arrière. Il se couvre d’abord, 
d'après Stepanoff, d'une cuticule, pour porter ensuite des cils et des cirrhes raides. Sa paroi sépaissit, et il s'en sépare intérieurement des cellules embryonnaires, qui deviennent les muscles de cet organe.

Ganine (cxхxix), dont le travail ne m'est malheureusement connu que par un extrait, ferait naître le pied chez Lymnceus, Planorbis, Physı, d'une nlanière qui rappelle ce qui a été observé chez Vermetus ef Calyptrrea : il ne faut peut-être pas attacher trop d'importance à cette information de seconde main, surtont puisque nous possédons le travail très-détaillé de Lereboullet.

La revue de tous ces résultats nous apprend d'abord que la forme la plus simple du pied est celle qui se rencontre chez les Lamellibranches. Iei le pied n'est bien positivement qu'une différenciation de la partie de la face ventrale de l'animal, comprise entre la bouche et l'anus. Chez les Céphalophores, le développement du pied est un peu plus compliqué. Les Pulmonés paraissent présenter le cas le plus simple, celui qui se rapproche le plus du type des Lamellibranches; le pied est ici aussi mue protubérance formée par l'ectoderme épaissi de la face rentrale, protubérance qui s'allonge d'abord en arrière, puis se met à saillir en avant par une croissance plus active de ceux des tissus qui avoisinent la bouche. Cette croissance peut avoir lieu d'une manière uniforme, ou bien elle peut être plus active sur les côtés (Lymnceus), de manière à former deux lobes, qui se soudent ensuite.

L'origine première du pied en forme de languette de la plupart des Céphalophores marins n'a pas ćté l'objet de l'attention de beaucoup d'observateurs. 11 est permis de croire, cependant, d'après les quelques données que j'ai recueillies sur ce sujet, que l'origine première de cette languette se trouve dans un épaississement cctodermique d'une grande partie de la région ventrale de l'embryon. Ce pied en forme de languette répond done, non pas an petit lobe postérieur qui porte l'opercule chez beaucoup de Céphalophores adultes, mais au pied tout entier, puisque les formations si diverses du pied des Céphalophores ne sont que des excroissances de ce pied primitif. L'extrémité de cette languette peut s'étirer en un prolongement filiforme ou en une lanière ciliće (Hétéropodes, Ptéropodes, Gymnosomes, Cymbuliacées, Creseis), qui peut même persister chez l'adulte (appendice caudal des Firolacées). A la partie antérieure et rers la base du pied, les tissus peurent bourgeonner et former un lobe situé sur la ligne médiane et bien distinct (nageoire des Hétéropodes); ou bien le bourgeomnement, la prolifération des tissus, peut s'adresser plutôt aux 
parties latérales (Vermetus), ou bien encore tout à fait aux bords latéraux (nageoires des Pléropodes). Tous ces appendices divers ont ceci en commun, qu'ils sont des cxcroissances du pied primitif. Mais ils ne sont évidemment comparables entre eux qu'en tant qu'ils poussent sur les mêmes régions du pied primitif. La nageoire des Hétéropodes ne peui donc être strictement comparée aux lobes antérieurs du pied de Vermetus ou de Lymnceus, et encore moins aux excroissances tout à fait latérales, qui donnent naissance aux nageoires des Ptéropodes, au pied élargi de Gasteropteron.

Lorigine première du pied de Vermetus et Calyptrcea ne paraît pas s'accorder avec ce que j'ai dit de son origine chez les autres Céphalophores et les Lamellibranches. Il sera bon toutefois, avant de se prononcer sur ce point, d'attendre les recherches futures, et de savoir si ce petit mamelon, qui se trouve au-dessous de la bouche, est bien l'origine de tout le pied, ou si ce n'est pas plutôt une saillie qui se forme de bonne heure, mais ne répondrait qu’à la partie supérieure de la région pédieuse.

\section{LA COQULLLE ET LE MANTEAU.}

Il est inutile de s'arrêter aux données anciennes, qui font dériver la coquille embryonnaire des Lamellibranches de la membrane vitelline, ou tout au moins d'une membrane continue, qui se fendrait ensuite en long; telles sont les données de Carus (xxi) et même de Karsch (LxII) et de de Quatrefages (Lxil1). 11 est cependant deux traits d'organisation des jeunes Nalades, qui n’ont pas échappé même aux auteurs anciens, à savoir : les crochets situés au bord des ralves et le byssus. L'enroulement de la coquille des Gastéropodes esl altribué par Carus (xI) et Jaequemin (xL) à la rotation de l'embryon, par Grant (xvi1) à un effet de recul produit par le choc du cœur.

La première apparition de la coquille des Céphalnphores esit décrite, d'une manière lout à fait concordante, par tous les auteurs qui s'en sont occupés. C'est une membrane plus ou moins bombée, en forme de verre de montre, qui apparaît au pôle aboral de l'embryou el va ensuite en croissant par les hords. Sourent cette membrane est pourvue de calcaire dès sa première apparition; d'autres fois clle est d'abord molle et ne süincruste qu'un peu plus taril. Je me dispense d’analyser en clétail toutes ces données.

Dumortier (xxxil) a vu, chez Lymnceus el Physa, une dépression 
mamelonnée de l'extrémité postérieure donner naissance à la coquille, observation qui s'explique par les résultats des travaux les plus modernes.

Lovén (xu et Liv), Sars (xın et LYu), Alder et Hancock (LI), Reid(LXIV), et surtout von Nordmam (LII) et C. Vogt (LxII) donnent la deseription de la coquille nautilö̈de des Opisthobranches et plus particulièrement des Gymnobranches, de la cavité branchiale qui s'ouvre à droite en arrière del'anus, de la manière dont la peau se détache de la surface interne de la coquille, sauf à son bord supérieur, assez longtemps avant que celle-ci tombe. Von Nordmann remarque que le bord supérieur de la coquille se confond avec le bourrelet du manteau. C. Vogt a ru le bord de la coquille dépasser le bord du manteau, auquel elle est attachée, et pense que cette portion de la coquille croît à la manière des ongles, de bas en haut. Cet observateur, si ingénieux du reste, n’a pas songé que les larves qu'il observait se trouvaient dans des conditions anormales et que le bord de leur manteau était plus retiré, que cela n'est le cas chez des larves à l'état naturel.

Lorén (Lx.xı) montre que la coquille apparaît chez Cardium sous forme de deux moitiés réunies entre elles du côté dorsal, mais encore dépourvues de charnière. Pendant que les valves grandissent, le repli du manteau, qui les tapisse, se sépare du corps.

Leydig (Lxxiv) montre que la cavité respiratoire de Paludina se forme par recouvrement du repli du manteau. le bord du manteau présente à un certain moment trois prolongements digitiformes, et la coquille a naturellement aussi les mêmes prolongements.

Van Beneden et Windischmann (xLIv) ont décrit déjà la formation du bouclier de Limax et l'apparition, dans son intérieur, des cristaux, origine première de la coquille rudimentaire de ce Pulmoné. 0. Schmidt (Lxxvı) et Gegenbaur (Lxx viII) confirment ces données. Ce dernier auteur surtout apporte, dans son fort beau travail, une foule de renseignements d'une haute importance pour l'histoire comparée de la formation de la coquille chez les Mollusques. Dans l'épaississement aboral de la peau, qui constitue le bouclier de l'embryon, apparaît une cavité sous forme de fente, parallèle à la surface, dans l'épaisseur même de l'organe. C'est dans cet espace qu'apparaissent chez Limax et chez Clausilia les petites plaques calcaires qui, par leur juxtaposition, constituent le rudiment de la coquille. Chez Clausilia la coquille s'agrandit rapidement; la couche de peau qui la recourre s'étend d'abord, puis s'atrophie en s'étirant et se déchire an milieu. Elle 
continue à être représentéc ici, comme chez Helix, et cela même chez les animaux adultes, par ce repli du manteau qui recourre le bord et remonte un peu sur la face externe de la coquille. Gegenbaur remarque que, chez les Pulmonés, il se dépose dans les cellules de toute la peau, surtout dans le derme, des concrétions calcaires abondantes.

C. Vogt(Lxxix et cviu) décrit et figure la coquille striée en travers de larres qui, je puis le dire, appartiennent à la famille des Hyaléacées. J'ajoute que la chute de cette coquille est un phénomène pathologique. L'auteur a vu et figuré les masses tuberculeuses qui se forment, après la chute de la coquille, dans la partie postérieure du manteau. Gegenbaur (ci) a vu le premier une larve de Ptéropode gymnosome dans sa coquille, mais il la rapporte au genre Creseis. Il a montré que les larves d'Hétéropodes sont munies d'une coquille, qui ressemble beaucoup à celle des Gymnobranches, coquille qui tombe ainsi que l'opercule chez Pterotrachora, tandis que Carinaria ne garde que la coquille et qu'Atlanta garde à la fois coquille et opercule.

Davaine (Lxxxyul) remarque que la charnière est la première partie de la coquille qui apparaisse chez Ostrcea; il eût été plus juste de dire ligament au lieu de charnière. 0. Schmidt (xcv) rapporte que chez Cyclas calyc. les jeunes embryons ont, sur les côtés de la partie ventrale, deux bourrelets garnis de cils qui se détachent librement en arrière, tandis qu'ils sont réunis entre eux en avant. Les ralves apparaissent au côté dorsal. Leydig a ru chez Cyclas cornea (cu) le manteau se détacher du corps sous forme de dewx replis latéraux, qui se forment d'arrière en avant. La coquille se montre sur le dos, pareille à une selle sur le dos d'un cheval. Puis apparaît la glande à byssus sous forme de deux petits follicules en arrière du pied. Les parties latérales de la pellicule, ou coquille primitive, se chargent de sels calcaires et forment les valves, reliées entre elles par la partie médiane de la pellicule, qui ne s'incruste pas. La glande du byssus atteint bientôt son plus grand développement; elle est pyriforme et sécrète des fils mous, homogènes ou finement striés, qui s’étirent comme de la salire et se cassent en formant une goutte terminale. De Lacaze-Duthiers (cin) pense que les fils du byssus des jeunes du Mytilus edulis ont une enveloppe plus dure que le contenu et constituent de véritables tubes.

Lovén (cıv) décrit avec précision le développement de la corpuille si singulière de Chiton. Le mantean, c'est-à-dire la peau du dos, se parlage par des sillons en sept articles, sur lesquels naissent autant do pièces lamelleuses ou valves. Ces valves ont de chaque côté une inci- 
sion à leur bord antérieur, incision qui s’efface plus tard; elles s’épaississent par des lamelles de plus en plus grandes, qui naissent entre l’épiderme et la lamelle précédente, qu’elles soulèvent. Les artieles antérieurs sont relativement plus larges que ce ne sera le cas plus tard. La huitiòme valie se forme plus tard, landis que les sept premières se montrent presque simultanément. Le manteau est garni à son borl rle petites épines raides. Ainsi done la valve postérieure n'est pas plus comparable que les autres valves à la coquille du reste des Céphalophores.

De Lacaze-Duthiers (cxu) a retracé, chez Dentatium, la première origine du tube dn manteau, à un petit sillon vertical, qui se montre à l'extrémité inférieure de la face ventrale de l'embryon. La coquille se montre au côté dorsal de l'extrémité inférieure, comme une petite écaille bombée et recourbée en cornet ouvert; elle est imprégnée de calcaire rlès sa première apparilion. Cette coquille crô̂t en s'évasant en haut; elle se rejoint an côté rentral, formant ainsi un entonnoir onvert des deux bouts. Le manteau aỵant fait le même mourement que la coquille, le sillon primitif s'est changé en un tube cilié, le tuhe palléal. Le manteau forme un bourrelet (1ui accompagne le bord de la coquille.

Claparède(cxin) a olsservé que, chez Neritina, le premier rudiment de coquille renferme du calcaire et présente des stries radiaires et des stries concentriques. La coquille grandit par les bords, qui sont sécrétés par le bourrelet du manteau, bourıelet composé d'un épithèle régulier. La cavité branchiale apparaît comme mo sillon, situé à la nuque, et qui va en s'approfondissant rapirlement.

M. de Serres (cxiv) remaryue que les Liamellibranches, tant de mer que d'eau douce, présentent, ponr la plupart, une usure de leurs nates (coquille larvaire) et une perte plus ou moins étendue de leur drap de mer (cuticule externe). Beaucoup de Gastéropodes marins ou d'eau douce abandomnent le somnet de leur coquille, qui s'use et s'exfolic; ceci s'explique par des raisons purement physiologiques, car l'animal, devenu gros, ne trouve pas dans le tortillon d'espace assez large pour s'y loger. Cés mènes particularités s'observent chez les genres fossiles, et en particulier chez des Céphalopodes des genres Turrilites, Hamites, Ancyloceras, ete.

Krohn (Lxxxy, cv el cxvı) déerit la singulière coquille nantiloüde, cartilagineuse, hyaline, munie d'épines et de facettes, de la larve de Marsenia, à laquelle il donnait d'abord le nom d'Echimosprira, et 
montre que le jeune animal, au moment où il quitte cette coquille larvaire, est muni d'une pelite coquille définitive. Macdonald (cxis) a observé des larves analogues. Cet auteur est, je rrois, le premier qui ait nettement établi l'homologie entre les coquilles internes et les coquilles externes des Mollnsques. De toutes manières, la coquille est le produit de la sérrétion du mantean ; seulement ce dernier s'inragine dans le premier cas, tandis que dans le second cas il s'étend à la surface. Malheureusement, ces vues très-justes n’étaient appuyées sur aucune preuve.

Chez Vermetus, de Lacaze-Duthiers (cxxul) fait olserver que, lorsque la coquille turbinée a atteint un certain développement, la cavité branchiale s'enfonce, surtout du còté droit, entre le bord de la coquille et le cou de la larve, ef cela non-seulement par un simple arrêt de développement de cette partie, mais encore par suite d'un retrait des tissus.

Krohn (cxxy) a montré le premier que les Ptéropodes gymnosomes passent par un prenier élat larvaire, où ils sont munis d'une coquille et d'un voile. La coquille de ces larves, au moment de la métamorphose, a la forme d'un cornet, terminé en bas par un ovoïde. La partie conique ou supérieure peut être lisse, ou striée, ou cannelée transversalement; elle peut acquérir une assez grande longueur avant la métamorphose, ou rester presque nulle et présenter de fines striures verticales. Les eoquilles des larves de Cymbuliacées sout lisses, enroulées dans un plan, rapidement élargies chez Cymbulia, moins ouvertes chez Tiedemannia; l'entrée de la cavité palléale se trouve à la nuque. Cette coquille larraire tombe, pour ètre remplacée par une roquille rartilagineuse, qui prend naissance dans l’épaisseur du derme. Les roquilles des Hétéropodes sont enroulées presque dans un plan; quelques-unes cependant sont un pen déjetées, tantôt d'un côté, tantôt de l'autre. Les coquilles des Firolacées sont peu enroulées, ct tombent lors de la métamorphose. Celle de Pterotrachica mutica est lisse, celle de $P$. coronata est cannelée transversalement.

Nous avons déjà vu que Lereboullet (cxxvu) prenait ì tort pour le rectum cet enfoncement à parois épaisses, qui se produit au pôle aboral chez. Lymnaus et qui sert de point de départ au bourrelet circulaire, origine du manteau. Nous verrons bientôt que Ganine a déjà releré celte erreur. Le savant embryogéniste français a du reste fort bien compris la nature et la signification de ce bourrelet circulaire, quoiqu’il se soit mépris sur son origine. Il montre que le pre- 
mier rudiment de la enquille apparait dans l'espaer eireonserit par le bourrelet, et que sa fonction est de sécréter la coquille, à mesure qüil s'avance du pôle aboral rers le pôle oral ; il a remarqué la clisposition régulière des cellules épithéliales qui le composent. Ce bourrelet remonte plus vile du côté dorsal que du côté ventral, où il reste plus ípais : il dévie anssi res's la droite. Chez Ampullaria polita, Semper (cxxis) a vu, au còté ventral et à gauche de l'extrémité poslérieure de l'embryon. un disque ovale, composé de cellules polyédriques régulières, lien limité en arant (côtć ventral) par un bourrelet, mais élalé en arriòre. C'est le disque coquillier, sur lequel la coquille ne tarde pas à se former. En môme temps que le disque coquillier, il se forme au côlć dorsal et droit de l'extrémité posté. lieure de l'embryon me petite excavation, origine de la carité pulmonaire. Cette cavité se trouvera plus tard, par suite de la croissance flu manteau et de la coquille, au fond d’une grande cavité palléale lans laquelle se dívelopperont les branchies. Dans la coquille, d'abord membraneuse, se déposent d'aborl des grains isolés, puis une couche continne de cristanx d'arragonile. De Lacaze-Duthiers a vu chez l'embryon d'Ancylus (cxxry), au côté opposé à la bouche, outre le disque pédieux, un second disque, qui est l'origine du manteau. II compare, arec beaucoup de juslesse, le mantean de la plupart des Géphalophores à un sac élastique, dans lequel senfonceraient les viscères en le distendant; chez Limax, le manteau restant très-petit. les viscères deseendent, dans le pied, qui aequiert de grandes proporlions. Toujours le manteau est Jien caractérisé, par le lait quil reçoit ses nerfs du centre nerreux inférieur.

Le manteau de Teryipes clav. est formé, d'après Selenka (cxxxy), de la conche externe ou cotoderme, lont les cellules se fusionnent, et qui montre encore, par-ci par-lì, les cellules étoilées et contractiles. Ces cellules servent, par leur contraction, ì détacher l'ectoderme de la copquille.

Chez Calyptraca sinensis, le manteau apparaît, d'après Salensky, (cxxxin), dès l’abord, comme un épaississement discoüdal de l'ectolerme, situé au dos de l'embryon, et au milieu duquel se forme aussitòt un petit enfoncement. Lat coquille se montre sons forme dun rerre de montre, athérent seulement au bord du disque. Le disque roquillier s’amincit en s'étendant, et ne conserve quelque épaisseur qu'au bold qui sécrite la coquille. La cavité branchiale se forme an ròté droit, entre le bord de la connille el te corps de la larre. Chez. 
Trochus rar. cet habile observateur a vu aussi le mantean naitre comme épaississement ectodermique au pòle postérieur, avec un enfoncement au milieu. La corfuille larvaire est séparée de l'ectoderme au fur et à mesure ([u'elle se forme. D'après Stepanoff (cxxхrui) la cavité pal léale de Calyptropa est dépourvue de cils, mais celle de Nassa reticul. en est garnie.

Ganine (cxrxix) a ru, sur les côtés tu pòle postérieur de l'embryon de Cyclas, se former deux enfoncements symétriques, qui sétalent ensuite, et sont l'origine du manteau. Le ligament se forme sur le dos qui sépare ces deux enfoncements. La glande à byssus se forme par enfoncement de l'ectoderme. Chez Lymnceus, Planorbis et Physa, le savant russe a vo un enfoncement infundibuliforme considérable se produire au pôle aboral; cet enfoncement, que Lereboullet a pris à tort pour le rectum, est, d'après Ganine, l'origine du manteau.

Chez Aplysia, E. Ray Lankester (CxL) rapporte que la "glande coquillière " est un des premiers organes qui se montrent à la surface de l'embryon. C'est un enfoncement de la surface, dont les cellules prennent un développement particuliel. Cette même glande se retrouve chez Pisidium. L'anteur ayant retrouvé cette glande chez Loligo, où elle serl à lormer la coquille interne de cet animal, en conclut que tous ces Vollusques ont un ancètre commun.

Les figures de Salensky (cxum) représentant des embryons d'Ostrcea edulis, montrent que l'enfoncement primitif du mantean se retrouve ici, et qu'il s'efface pour former la coquille en forme de selle.

De toutes ces données très-concordantes, il résulte que la première origine de la coquille se trouve dans un épaississement de l'ectoderme, épaississement qui s'enfonce dans son centre. Cet enfoncement paraît très-répandı, puisque je l’ai moi-même rencontré chez les Hétéropodes, les Ptéropodes el Limax; il a été observé en outre chez des Gymmobranches et diver's Lamellibranches. Cet enfoncement est le point de départ d’un bourrelet, qui sécrète le bord de la coquille externe, ou d'une cavité, où se forme la coquille interne. La coquille elle mème varie de forme, sans doute, mais elle est unirque à l'origine, chez les Lamellibranches comme chez les Solénoconques el les Céphalophores.

Il est à noter aussi, tuoique cela ne soit pas nonvean, que tous res Mollusques, sans une seule exception, possèdent une coquille, au moins dlans leur état larvaire. 
LA CAVITÉ DU CORPS ET LES MUSCLES.

Sur le mode de formation de la carité du corps, lous les auteurs sont d'accord : c'est un espace qui se forme par l'écartement qui se produit entre l'ectoderme et l'entoderme. Chez Aplysina, qui possède d'après Stuart (cxisx) un mésoderme, au moment où une partie de la sphère cellulaire s'infléchit pour former l'entoderme, la portion correspondante de ce mésoderme s’infléchit en même temps, et la cavité du corps se forme entre ces deux feuillets mésodermidues. Chez C'yclus (sp. ?), il se forme d'après Ganine (cxxxix) une couche mésodermique compacte, qui occupe tout l'espace entre l'ectoderme et l'entoderme. Cette rouche se scinde ensuite en deux fenillets, dont l'un reste appliqué au feuillet interne, l'autre an fenillet externe de l'embryon, el la carité du corps résulte de cette scission.

Dans la grande majorité des cas, l'entoderme et l'ectoderme se sćparent l'un de l'autre, arant que le mésoderme soit formé autrement que par places. Les deux feuillets moyens paraissent alors se former l'un à la face interne de l'ectoderme et l'antre à la face externe de l'entoderme, après que cette séparation a eu lieu el par scission de ces deux couches de l'enbryon.

L.es premières tibres musculaires prennent naissance au moment nù les deux feuillets primitifs se séparent l'un de l'autre; on les considère généralement comme des points d'adhésion de ces feuillets. ou romme des cellules qui les relient, et qui sont étirées par le fail de leur séparation. Ces fibres, évidemment contractiles, qui relient les riscères aux parois du corps, ont été vues par les plus anciens auleur's, qui se sont occupés du développement des Mollusques marins. On les considère généralement comme provenant de l'ectoderme. Les fibres ramifiées du voile ne sont qu'un cas particulier, qu’un développement spécial de ce système de fibres. Elles ont toutes à l'origine mn nucléus et répondent, histologiquement parlant, chacune à une cellule.

Le développement du muscle rétracteur ou columellaire, des larves de Céphalophores à coquille externe, a été assez négligé par les embryogénistes. Tes auteurs qui s'en sont occupés le font provenir d'un amas de cellules, situées à la face interne de l'ectoderme, près du sommet de la coquille. Stuart (cxis) rapporte que chez Aplysia cet amas se composerait de bonles très-réfringentes. L'amas s'étirerail à la reneontre dusecond point d'insertion du muscle, et les boules qui 
le composent, se diviseraient en petits corpuscules réfringents, qui se mettraient en lignes longitudinales el formeraient ainsi de belles fibres musculaires striées. Cette observation n’a encore été confirmée par personne. Tout au contraire, les antres observateurs s'accordent à ne voir, dans le muscle columellaire, que des fibres homogènes, ou tout au plus finement striées en long. Sclenka (cxxxy) a vu ce muscle, chez les larves de Tergipes clav., composé de fibres ou bandes homogènes. à la surface desquelles il a rencontré encore par-ci par-là des cellules embryonnaires nucléées; il pense que le muscle tout entier se forme aux dépens d'une quinzaine de cellules.

Jacquemin ( $\mathrm{x}$ ) avait déjà remarqué que le point d'insertion du muscle columellaire avance par la formation de nouvelles fibres au côté antérieur, tandis que les fibres du côté postérieur se détachent et disparaissent.

Plusieurs des larves de Ptéropodes gymnosomes observées par Gegenbaur ( $\mathrm{al}$ ) et par Krohn ( $\mathrm{cxxv}$ ) possèdent, outre le muscle columellaire, situé du côté dlorsal, un second muscle, qui part de l'extrémité postérieure de la coquille, vers la face ventrale, et se perd à la hauteur de l'estomac. Sars (Lvil) et Reid (Lxiv) ont déjà remarqué que, chez les Gymnobranches, l'une des fibres qui relient la masse viscérale à la paroi du corps est particulièrement forte; e'est celle qui s'étend de l'estomac à la partie inférieure de la coquille. Le rapprochement entre ces deux observations est facile à faire.

Chez l'embryon d'Ampullaria pol., Semper (cxxxı) a ru un muscle court, qui s'étend du milieu du pied à la masse hépatique.

Sur le mode de formation des museles qui servent à fermer les valves de la coquille des Lamellibranches, je n'ai pu recueillir aucun renseignement. Chez Cardium et Montncuta, e'est, d'après Lovén (Lxxi), le muscle antérieur qui se forme le premier ; chez Mytihus edulis, d'après de Laeaze-Duthiers (cvil), le muscle postérieur apparaît avant l'autre, et ce fait explique comment certains Lamellibranches, tels que Spondylus, Pinna, Ostrcea, n’ont qu'un muscle répondant au muscle postérieur des Lamellibranches dimyaires; ce serait un simple arrêt de développement.

Ganine (cxxrix) rapporte que les lils qui relient chez Lymnceus, Planorbis, Physa, le côté dorsal de l'ectoderme aux viscères, proviennent de l'entoderme. Ce n'est pas ce que j'ai ru chez Limax.

Les muscles proviennent du mésoderme chez Cyclas, d'après le même auteur. Il n'est peut-être pas inutile de noter que chez la plu- 
part des Céphalophores, du moment que la cellule qui doit s'étirer et lomner naissance à une fibre musculaire s'est détachée de la face interne de l'ectoderme primilif on de la face externe de l'entoderme primitif, elle appartient théoriquement au mésoderme. Il n'y a entre les deux cas qu’une différence : chez, les Mollusques à élat larvaire caractérisé, le développement est abrégé, et les organes se forment de la manière la plus directe et la plus expéditive, tandis que chez ceux qui ne passent pas par un élat larvaire véritable, les cellules embryonnaires constituent d'abord un véritable feuillet moyen, où les organes mésorlermiques s'esquisseront peu à peu. Les Céphalopodes sont dans ce dernier cas.

LES ORGANES DE CIRCULATION ET D'EXCRÉTION L.IRVAIRES.

Cies organes tramsitoires sont si apparents chez quelques Pulmonés. surtout chez le genre Limax, qu'ils ne pouvaient échapper à l'obserration des auteurs, même les plus anciens, qui se sont occupés de l’embryogénie de ce Mollusque.

Laurent (xxrui) remarque que l'embryon de Limax mufus et flavus présente de bonne heure une vésicule antérieure, transparente, réticulée, contractile, et une vésicule postérieure, qu'il nomme la rame caudale, également contractile. Ces deux vésicules exécutent des monrements manifestes. Dans l'intérieur de la vésicule (céphalique), l'on voit une bande transrersale parsemée de points noirs, recourbée sur plle-même et envoyant ses deux branches en arrière. Il considère cet organe comme la glande rénale. Cette bande rentre, ainsi que la vésicule antéricure, sous le bouclier, et la rame caudale diminue et disparaît. Plıs tard (xxxı) le même auteur remarque que les deux vésicules se contractent alternativement et se renvoient le liquide qu'elles contiennent. La vésicule (céphalique) se retrouve chez Limax agrestis sinereus, Helic nitida ef Paludina vivipara. Van Beneden et Windischmann (xur) confirment en somme ces donnces ; ils constatent que la vésicule caurlale se forme ì l'extrémité du pied le l'embryon, et que les deux vésicules ne disparaissent quaprès la formation du cour. en solte que les deux genres de circulation coexistent pendant un certain temps. Ils ne se prononceut pas sur la nature du uruban gramulé contourné en $S$ '

C. Vogt (Lxu) a vu, chez Actewn, le pied se gonfler of se resserrer tour à four, et cescontractions alterner aver celles de l'intestin. II y 
a erreur sur ce dernier point ; ce n'est pas l'intestin, ćest la paroi interne de la cavité branchiale, qui renvoie le liquide chassé par le pied. L'auteur conclut, du reste, arec justesse que ces larves présentent une circulation lacunaire avant l'apparition du cour.

Leydig (Lxxiv) confirme le fait des contractions alternatires du pied et de la région antérieure chez Paludina vivipara. Ces contractions se font à raison de quatre à cinq à la minute et cessent arec l'apparition du ccur.

0 . Schmidt (Lxxv) remarque que la résicule caudale a des parois minces, et que sa contractilité est due à des cellules musculaires étoilées, qui la traversent en tous sens; il en est de mème de la vési cule antérieure, qu'il nomme vitelline, confondant les deux couches qui la composent. La vésicule antérieure possède sur ses côtés le tube recourbé, vu par Laurent, tube à parois homogènes, et tapissé intérieurement de cellıles à noyaux verdâtres. Ciet organe se continue en haut en un tube excréteur, yui se perd sous le bouclier ; il ne grandit guère, et disparaît à la fin te la vie embryonnairc, en commençant par le tube excréteur. L'auteur compare cet organe au corps de Woll. La vésicule antérieure se courre d'mne peau épaisse et cesse de se contracter ; il en est de mème de la résicule caudale, mais les deux tentacules se contractent alternativement, produisant ainsi un déplacement des fluides. Le cour, pendant ce temps, est entré en activité. Gegenbaur (Lxxvul) relève aver justesse le fait que les deux vésicules de Limax ne sont que des régions de lat peau devenues contractiles par le fait du dércloppenent de cellules allongées, ramifiées, contractiles et dirigées perpendiculairement à la surface de ces organes. A la surlace de la vésicule vitelline (sac nutritif, Rel.), mais sons la conche contractile, se différencic de bonne heure l'organe recomrbé que le célèbre anatomiste u'hésite pas à considérer comme une glande rénale d'existence passagère. C'est d'abord un amas de cellules claires, qui se prolonge vers la tête; puis apparaissent dans les cellules de petits grains verdàtres, et le canal excréteur sallonge et rient déboucher sous le bouclier. Les cellules de cet organe sont dabord nucléées ; puis il se forme, dans leur intérieur, une vacuole, qui grandit au point de repousser le protoplasme et le nucléus de la cellule primitive tout contre sa membrane. C'est dans cette vacuole (Secretbläschen) que se montrent les granules, verdatres à la lumière transmise, jaunes à la lumière incidente, qui dénotent le ròle phỵsiologique de l'organe. Plus tard le canal excrétemr disparait, et la gelande 
se réduit à un amas verdâtre. Ces mèmes organes larvaires, vésicules contractiles et rein primordial, se retrouvent chez Clausilia el Helix, mais moins développés que chez Limax.

Koren et Danielssen onl décrit (Lxxx) le sinus contractile des larves de Buccinum et Purpura, qüils prennent pour le cœur. Ce sinus est situé derrière le bord postérieur du voile; il est ovale et fait saillie à la surface. Ses parois possèdent d'abord des fibres musculaires longitudinales, puis des fibres transversales. Ces fibres seraient de véritables petits tubes élargis par places. Le sinus fait quarante à soixante pulsations à la minute. Lorsque le rebord du manteau vient à couvrir la nurgue de l'animal, il recourre aussi le sinus, qui se trouve ainsi dans la cavité branchiale. A Carpenter (cri) et à son collaborateur M. Busk revient le mérite d'avoir démontré que cette vésicule nuchale n’est pas le cour, el que ce dernier se tronve beaucoup plus bas.

Les contractions de la paroi du corps, que Vogt (Lxxix) a observées chez ses larves (de Hyaléacées) après la chule de leur coquille, sont un phénomène pathologique, que j’ai observé anssi dans les mêmes circonstances. J. Miiller (cx.xum) décri très-bien le sinus contractile dorsal de la larve des Creseis acic.; son existence est due à la contractilité de la membrane, qui sépare le sac viscéral de la carité branchiale. Il s’étend depuis le voile, en haut, jusqu'au niveau de l'estomac. C'est à torl que Gegenbaur (cı), à quil l'existence de ce sinus a échappé, croit pouvoir l'identifier avec la poche rénale contractile. J. Müller (cxis) maintient ses données el reconnait dans cet organe non plus un simple ventilateur de la cavité branchiale, comme il l'a vait cru d'abord, mais un organe de propulsion du liquide nourricier. Il montre que ses contractions sont ondulantes et dirigées de bas en haut.

Les jeunes de Dentalium n'ont, d'après de Lacaze-Duthiers (cxiI), pas encore de cœur à l'âge d'un mois. Le pied, en se contractant, envoie le liquide nourricicr qu'il renferme dans le sinus abdominal, qui se dilate. Lursque celui-ci se resserre à son tomr, le pied se gronfle. La contraction de ces sinus est lente, leur dilatation est subite, comme une détente. Le sinus abdominal communique directement avec l'extérieur, c'est-à-dire arec la cavité palléale, par deux onvertures qui peurent se fermer complétement. Chez les larves de Vermetus, d’après le même auteur (cxxus), il n'y a pas de sinus contractile différencié; mais la paroi du corps délachée de la coquille est éminemment contractile et remplit la fonction du déplacement des fluides.

Semper (cxxxu) n'a observé chez. Ampullaria polita qu'un petit sinus 
larvaire; c'est une petite étendue de l'ectoderme située entre le pied el la protubérance postérieure, ¡ui montre ces battements. Les autres parties du corps et du pied de l'embryon muntrent bien des contractions vagues et irrégulières, mais le sinus, qui a été décrit, est le seul endroit qui présente des contractions rhythmirques. Le même auteur a retrouré chez Bulimus citrinus le mème petit sinus que chez Ampullaria. Fayinulus luzonicus n'a pas le moindre sinus contractile, pas plus que Paludina costata; chez cette dernière, cependant, les tentacules se développent de bonne heure, ef sont relativement bien plus grands que chez l'adulte. Ces tentacules se contractent alternativement et produisent ainsi le déplacement des fluides. Chez une espèce vivipare du genre Vitrina, ce naturaliste distingué a observé deux résicules contractiles, à la nuque el à l'extrémité du pied, très-semblables à celles de Limax. Les espèces du genre Melania, enlin, sont toutes riripares: elles passent par un état larvaire bien caractérisé, arec un voile très-développé, mais sont dépourvues d'organes de circulation larraire. Chez Vitrina, et surtout chez Bulimus, il y a bien, dans toute l'étendue de la cavité du corps, un réseau de museles anastomosés, mais ces muscles ne montrent que des contractions irrégulières, qui ront en augmentant avec l'âge, tandis que les sinus contractiles montrent des battements rhythmiques, qui diminuent et cessent dans la suite du développement.

Salensky (cxxxvis) a montré que, chez Calyptrea, l'espace circonscril par le bourrelet du voile se soulève, pour former ce qu’il nomme la résicule céphalique: mais il ne paraît pas que cette résicule ait des contractions rhythmiques. En revanche, le même animal possède à l'étal larraire un véritable sinus contractile, qui fait à la nuque, en arrière du voile, une saillic de la forme d'un œuf fendu en long. Ce sinus, ou cocur larraire, esl placé en travers; il se compose de la conche ectodermique et de fibres musculaires, transversales par rapport à l'animal, mais longitudinales par rapport au sinus lıi-même. Ces fibres ont des bords parallèles, et ne sont élargies cu'au milieu de leur longueur. Il est évident que ce sinus est l'homologue de celui qui a élé déerit chez Buccinum et Purpura. L’auteur le compare aussi à ia lıuque contractile de Paludina, et fait observer qu'il ne se retrouve pas chez tous les Prosobranches; il se trouve chez Nassu retic., mais manque à Vermetus el à Veritiur. Le sarant russe dévelople son opinion, d’après laquelle ce sinus contractile n'est pas comparable à la résicule antérieure des Pulmonés, parce que cette dernière possède des 
tibres perpendiculaires à la paroi, est ciliée à la surface, et présente des contractions régulières; landis que le cour larvaire, de ceux des Prosobranches qui en sont pourrus, n’a que des fibres accolées à la paroi, est glabre, el se contracte irrégulièrement. L’homologne de la résicule antérieure des Pulmonés se trouverait dans la résicule céphalique courerte de cils de Calyptrcea, et les muscles de la première seraient comparables aux muscles du voile de ce Prosobranche. Je développerai plus bas les raisons qui m'empêchent de partager cette manière de voil.

Entre le ewur larvaire et la résicule céphalique de Calyjptrrea sinensis et de Nassa retic., se trouvent, toujours d'après Salensky, quatre cellules énormes situées à la surface, el composées d'un nucléus, d'un contenu protoplasmique et d'une vésicule d'excrélion, telle que (iegenbaur en a décrites, renfermant une ou deux conerétions urinaires et un liqquide jaunatre. Ces cellules n'ont pas de canal excréteur; elles commenceul par se multiplier, pour tomber plus tard, en même temps que le cœur larvaire disparaît. La description que donne Stepanoff (cxxxyin) du ceur larvaire de Calyptrapa sinensis et de Nassu reti: concorde avec celle de Salensky, sauf uque ce sinus serait courert de cils vibratiles à sa surface.

Ganine (cxxxıs) déerit, chez Lymnceus, Planorbis et Physa, des glandes rénales provisoires. Ce sont des cellules paires, très-grosses, prove. nant de l'ectoderme, el situées derrière le bord dorsal du voile; elles débouchent dims la cavité du corps par de longs canaux excréteurs, el ne disparaissent quiaprès la formation du rein définitil.

Etablir les homologies entre tous les organes larvaires que nous venons de roir n'est pas chose facile, ni même possible, dans l'état atctuel de nos comnaissances. Le rein primordial des Pulmonés lerrestres, situé entre l'ectoderme el l'entoderme, el débouchant entre lu manteau et le pied, près de l'anus, ne peut certainement pas se comparer aux cellules rénales superficielles, situées à la nuque de quelques Prosobranches el de quelques Pulmonés d'eau douce.

Entre la vésicule céphalique des 'Julmonés terrestres el les sinus du pied el de la nuque de dirers Céphalophores, la comparaison n’est pas non plus possible, à moins que des données nouvelles sur les relations de ees parties arec les organes roisins ne viennent fournir des points de repire. L'est ce que j'essayerai de faire plus loin.

Jusqu'à prísent, aucun organe de circulation ou d'excrétion provisoire n’a été ubservé che\% les Lamellibrinches. 
Le grand développement que peurent prendre les organes larvaires de propulsion, dans les cas où le voile est nul, et leur développement restreint dans les cas où le voile est bien développé, tendent bien à montrer que les contractions fréquentes du voile, chez les véritables larres, ont pour effet de mettre en mourement les liquides nourriciers. A ce point de rue purement physiologique, mais non pas au point de rue morphologique, le roile des uns peut se comparer anx sinus eontractiles des autres.

LH COEUR ET LA GLANDE RÉNALE OU ORGANE DE BOJANUS.

Pour ces organes, comme pour ceux que nous avons déjà passés en revue, je m'attacherai plutôt à recueillir toutes les données utiles, qu'à suirre une méthode purement historique. Carus et Grant passent pour avoir les premiers discerné le ccur chez les larres de Mollusques.

Jacquemin (xL) fait naître le cœur, chez Planorbis, de deux amas de globules, qui se creusent pour donner naissance aux deux chambres. D’après van Beneden et Windischmann (xL1v), le cour de Limax se forme d'une seule cavité, qui se sépare en deux par un étranglement; après quoì le péricarde se forme alentour. Le rein naît comme cæcuu à côté de l'anus. Von Nordmann(Lui) constate que le cœur n'apparaîl chez Tergijues Edu'. qu'après la métamorphose; chez Terg. lacin., il se montre quatre semaines après l'éclosion, d'après Max Schultze (Lxxu). Lovén (uv) a vu, chez les Bullidées, un organe vésiculeux se former à còté de l'anus, organe qui ne peut guère être que le rein. Les résultats négatifs de Sars (Lvi) et de Vogt (Lxi), dans leur's efforts pour trouver le copur des larres de Gymnobranches, montrent que l'apparition de cet organe est au moins anssi tardive ici que chez les autres Mollusques. Liorgane résiculeux contractile, que Reid (Lxiv) a trouvé à côté de l'anus dles Gymnobranches, ne peut guère être que la glande rénale.

Les larves de Lamellibranches élevées par Lovén (Lxxı), même les plus âgées, n’avaient pas encore de crur ; mais des larves d'espèces indéterminées, prises en mer, possédaient déjà l’organe de Bojanus sous forme de deux vésicules paires, transparentes ou à parois granuleuses, contractiles et renfermant quelquefois elles-mèmes de petites résicules pleines de granulations.

Chez Limax, 0. Schmidt (cxxvi) a ru, à còté et eu arrière du péricarde. apparaître un anıas de cellules à graiıs verts, le rein véritable. 
Gegenbaur (Lxxyin) a vu le cœur se différencier d'un amas de cellnles situé au côté dorsal; il se contracte d'abord au milieu, qui répond à l'ouverture entre le rentricule et l'oreillette, et se sćpare ensuite en deux chambres. Dès qque le cœur bat, le rein se montre derrière l'oreillette, son canal excréteur débouchant à côté de celui du rein primordial de droite. Les cellules, qui composent la paroi de l'organe, subissent la mème séric de modifications que celles du rein primordial. Chez Helic et Clausilia, la formation de ces organes est la même. Chez ce dernier genre, le soigneux observateur a ru le cœur s'étirer par places, pour domner naissance à l'origine des vaisseaux, et sa paroi se différencier en une couche interne de cellules musculaires étoilćes, qui vont d'une paroi à l'autre, et en une couche externe en forme d'épithélium.

Koren et Danielssen (cxxx), après avoir pris le cœur larvaire de Buccinum et Purpura pour le cœur véritable, ont pourtant bien vu ce dernier, qui, dans leur opinion, dériverait du premier. C'est à côté du cœur qu'ils ont vu une vésicule à parois minces, à pulsations régulières, munie d'un long tube excréteur, qui accompagne l'intestin ; cette vésicule est la glande rénale, ainsi qu'ils l'indiquent fort bien. Plus tard, Carpenter (cvi) a vu le vrai creur se former près du fond de la cavité branchiale.

J. Müller (Lxxxui) a vu, chez les larves de Cleodora (Creseis acic.), le cœur ct le rein apparaître en même temps, ce dernier sous forme de poche contractile, qui s'étend de l'oreillette à la cavité palléale, et possède une ouverture ciliée. Cet organe a étć vu par Eydoux et Souleyet, qui le nomment la poche pyriforme. Plus tard lc même auteur (xcril) et Gegenbaur ( $\mathrm{Cl}$ ) montrent que cette poche rénale a deux ouvertures, dont l'une débouche dans le péricarde et l'autre dans la cavité palléale. Ce dernier observateur ajoute que la poche rénale bat aussi vivement que le cneur, mais pas d'une manière synchronique, è fait voir que la structure de cel organe est la même chez les Hétéropodes. Krohn (cxxv) a confirmé plus tard ces résultats, mais aucun de ces aulteurs ne nous donne le moindre renseignement sur le mode de formation du cour et du rein, ni chez les Ptéropodes ni che\% les IIétéropodes.

Leydig (cu) a vu les deux organes rénaux avec leurs concrétions chez les jeunes de $C$ yclas cornea: le cour ne se montre que très-tard. Davaine (Lxxxviu) avait eru reconnaître le creur chez des larves d' $O s-$ trcea edlutis avant leur sorlie des branchies de la mère. mais de Lacaze- 
Duthiers a montré que ce doit être une erreur. Ce savant distingué a suivi le déreloppement des larves d'Huîtres (xcrv) pendant plus de quarante jours après leur naissance, sans que le moindre rudiment du cour y apparût. Chez les jeunes de Mytilus edulis, le même obserrateur (Cvu) dérrit les vésicules rénales transparentes, situées devant le muscle postérieur dés valves, et se contractant à de longs intervalles. Chez Dentalium (cxit), le conur ne se montre pas encore mn mois après la naissance. Les organes de Bojanus apparaissent sous forme de deux petits amas, des deux côtés de chacune des ouvertures qui font communiquer la carité du colps avec celle du mantean; ils sont donc au nombre de quatre en tout, disposés en carré autour de l'anus. Ces amas s'épaississent, se lobent, et les lobes se creusent; leurs canaux excréteurs n'ont pas pu êlre découverts.

D'après Lereboullet (cxxviı), le cœur de Lymnceus se montrerait dès l'origine sous forme de double vésicule creuse. Semper (cxxxri) a vu chez Ampullaria le rein apparaître sous forme de concrétions miques, disséminées dans les cellules situées entre le cœur et le bord du manteau, mais sans former de glande bien définie, pas plns que ce n'est le cas chez l'adulte.

Chez Calyptrrea sin., d'après M. Salensky (cxxxrit), le rein est un amas de cellıles provenant du repli palléal, qui couvre la cavité respiratoire, cellules qui renfermept des vacuoles pleines d'un liquide jaune. Le cœur se forme dans le mésoderme à côté et en même temps que le rein; les cellules de ce feuillet s'allongent et se disposent autour d'un espace orale, qui devient la cavité du péricarde. Ce péricarde se resserre, et le cœur y apparaît vers la fin de la vie larvaire, sans que l'auteur sache de quelle manière il se forne. Stepanoff, au contraire, voit le cœur apparaître, avant le péricarde, comme corps ovale, solide, yui se creuse ensuite et se sépare en denx chambres. Le rein se forme à côté, mais sa communication avec le péricarde n'a pas été oloscrvée.

Ganine (cxxxux) rapporte que le rein se forme chez Cyclas aux dépens du feuillet externe, le cœur et les vaisseaux sanguins aux dépens du feuillet muyen ou mésoderme. Chez Lymnceus, Planmrbis, Physa. les éléments mésodermiques forment d’abord une vésicule: le péricarde. Dans celte vésicule, à son côté dorsal, se montre un amas cellulaire solide, qui se sépare en deux lobes et se creıse, pour former le ventricule et l'oreillette. I.es cellules intérieures de l'amas se détachent pour former les corpuscules du sang; les vaisseanx sanguins se forment en lieu et place. Le rein se forme chez les Lamellihran ches 
(Cyclas?) comme chez les Céphalophores (Pulmonés?) par invagination du feuillel externe; il est pair, infundibuliforme, puis vésiculeux, chez Cyclas; il est solide à l'origine chez Lymnceus et autres Pulmonés.

La plupart des auteurs qui ont décrit les larves de Gymnobranches ont vu, à coté de l'anus de ces larves, deux petits corps cellulaires attachés à l'ectoderme. Langerhans (CxLI) décrit, au côté aboral de l'anus, chez Acera, un corps rond déjà vu par Lovén chez Bullexa. Il se compose de cellules cunćiformes, pleines de gouttelettes très-réfringentes. L'auteur, ayant vu ces cellules vider leur contenu à l'cxtérieur, ne doute pas qu'il ne s'agisse d'un organe exeréteur. Doris et $L$ olis possèdent le même organe. Au côté dorsal, immédiatement sous le bord de la coquille, se trourent deux vésicules symétriques, assez grandes, avec une grande cavité vibratile renfermant des concrétions jaumes. La nature de cet organe est restée inconnue.

Résumer ces résultats et en tirer des conclusions me paraît chose impossible pour le moment, du moins en ce qui concerne le cœur. Quant au rein, il semble qu'il se forme aux dépens de l'ectoderme, et cela, dans la plupart des cas, dans le voisinage de l'anus. Ce fait est établi sur des données bien peu nombreuses, il est vrai ; mais aucun observateur ne rapporte positivement qu'il ait vu le rein se former aux dépens d'un autre feuillet.

\section{LES BRANCHIES ET LA CAYITÉ PULMONAIRE.}

: Sur le mode de formation de ces organes, tous les embryogénistes qui s'en sont occupés, sont d'accord; je serai donc très-bref.

Lovén (Lxxi) a vu les branchies se former, chez des larves de Lamellibranches prises en mer, comme bourrelet plissé du manteau, dans l'angle interne qüil forme avec le corps. Ces plis deviennent ensuite des digitations, dont la cavité interne reste en communication avec celle du corps. Leydig (ciI) rapporte que, chez Cyclas cornea, les branchies naissent comme processus du manteau, qui apparaissent successivement d'arrière en avant. De Lacaze-Duthiers (cvi), dans son travail si clair et si précis sur la formation des branchies de Mytilus edulis, montre que les processus digitiformes du manteau, vus par Lovén, ne sont que l'origine du feuillet direct de la branchie interne. Ces processus sont renflés au sommet; lorsque les digitations ont atteint une certaine longueur, les renflements se soudent entre 
eux, et forment un bourrelet terminal continu. De ce bourrelet part la lame réfléchie, qui se perce presque aussitòt d'une série de fentes répondant aux fentes de la lame directe. Les deux lames d'une même branchie se forment donc par des procédés fort différents. La branchie externe naît plus tarl que la branchie interne, et en dehors de la première, mais par le mème procédé, à cette seule différence près, que les premières digitations se montrent en un point plus éloigné de la bouche, en sorte qu'elle devra s'étendre non-seulement d'avant en arrière, mais encore d'arrière en avant. Les lames directes des deux branchies sont en contact l'une avec l'autre; les lames réfléchies se recourbent, l'une vers le corps, l'autre vers le manteau.

Leydig (Lxxiv) montre que, chez Paludina vivip., les branchies naissent comme bourgeons allongés, solides, dans la cavité palléale; ils se creusent ensuite intérieurement et deviennent très-contractiles, quoique renfermant peu de fibres musculaires. Koren et Danielssen (Lxxx) rapportent que la branchie de Buccmum apparaît sous forme de bourrelet dans la cavité branchiale ciliée, bourrelet qui donne naissance à des tubes tordus en spirale, ciliés à leur bord interne chez Purpura, et munis plus tard de fibres musculaires. Chez Termetus, la branchie se montre, l'après de Lacaze-Duthiers, comme bourrelet solide, dans l'épaisseur de la roùte du manteau; ce bourrelet est situé du côté gauche, porte des cils puissants, et affecte la forme d'un fer à cheval. Stepanoff (cxxxvii) voit la branchie rle Calyptrcea sin. naître comme protubérance de la paroi interne du repli du manteau, protubérance sur laquelle poussent les lamelles, sous forme de mamelons compactes. Les lamelles du milieu apparaissent les premières; elles se creusent successivement, ainsi que la protubérance branchiale, et toutes ces cavités entrent en communication entre elles et avec la cavité du corps. Les lamelles deviennent contractiles. Salensky (cxxxvn) indique clairement que les branchies de Calyptrcea résultent d'un épaississement de l'ectoderme de la cavité palléale. Ganine (cxxxis) montre que Paluelina, Bythinia et beancoup de Prosobranches ont du côté gauche le rudiment d'une seconde branchie, qui n'entre jamais en fonctions.

De Lacaze-Duthiers (cxn) prouve que Dentalium ne posside de branchies à aucune période de son existence; sa cavití palléale est, du reste, tapissće de cils. Les larves d'Entoconcha décrites par J. Miiller (Lxxiı) n'ont pas de branchies, mais leur cavité branchiale présente deux rangées de cils, dont liume est longitudinale ef suit la 
courbure de la coquille, tandis que l'autre est transversale et rapprochée de l'ouverture de la coquille, à laquelle elle est parallèle. Le même auteur montre que Creseis acir. possède, sur les parois de sa cavité palléale, une quantité de petiles touffes de cils disséminées. Huxley (xal) prend le bouclier ciliaire de la cavité palléale des Ptéropodes, déjà fort bien décrit par Eydoux et Souleyet, pour nne branchie rudimentaire. Gegenbaur (cr) montre que la branchie réritable des Ptéropodes Gymnosomes, dont Eydoux et Souleyet avaient fort bien compris la signification, se tronve au fond de la cavité palléale et que le bouclier ciliaire n'a rien à f'aire avec la branchie. La branchie des Ptéropodes affecte, du reste, une lorme qui est larvaire pour les autres Céphalophores, puisqu'elle se présente sous forme d'un simple cordon plissé et cilié, montrant tout au plus des rudiments de lamelles. Elle manque aux Thécosomes de forme trèsallongée. La branchie latérale de quelques larves de Gymnosomes est. à mon sens, peut-être morphologiquement comparable à celle des antres Mollusques; mais la branchie terminale de Pneumodermon ne saurait l'être. J. Müller (cxıx) insiste encore sur le fait que le bouclier vibratile des Thécosomes est muni d'une veine et sert à la respiration. Krohn ( $\mathrm{cxxy}$ ) a retrouvé le bouclier vibratile chez les larves du genre Cymbulia.

Gegenbaur (Lxxvir) a ru, ehez Limax el Clausilia, le ponmon se former comme enfoncement à parois ininces, sous le bord du manteau à droite. Lereboullet (cxxviı) roit, chez Lymnous, une excavation à parois transparentes pousser de l'extérieur entre le pied el la partie postérieure du corps; c'est l'origine de la cavité pulmonaire, dont l'entrée est ensuite recouverte par le manteau, qui s'arance en forme de voûte. Semper (cxxxul) décrit, chez l'embryon d'Ampullaria polita, un petit tron situé au côté rentral et à droite de l'extrémité postérieure, ouverture au-dessous de laquelle se trouve un amas de cellules. 'Telle est l'origine du sac pulmonaire, dont l'ouverture débouchera plus tard au fond de la cavité branchiale, formée par le recourrement du manteau. De la paroi de cette dernière cavité naissent ensuite les feuillets de la branchie.

Ces résultats sont trop concordants pour qu'il soit nécessaire de les résumer. 
LES ORGANES DES SENS.

Les otocystes ont été rus et elairement décrits, ‘hez l'embryon de Lymnée, par Pouchet (xxxru), qui parle des gramules (otolithes) se culbutant dans leur intérieur. Leur véritable nature a été comprise par van Siebold (XLY), par van Beneden et Windisehmann (xLIV) et par Sars (LTil). Ils ont été revus depuis chez tous les Céphalophores et tous les Lamellibranches, qui ont été étudiés, et tous les auteurs, à l'exception d'Allman (Lx) et de Reid (Lxiv), ont reconnu leur véritable nature. Partout ces orgaues se présentent sous forme de vésieules, rondes un ovales, à parois épaisses et à contenu liquide, dans lequel apparaît un otolithe rond. Elles sont situées latéralement un peu au-dessous de l'entrée de l'wsophage. L'on a sourent réussi à distinguer les cellules, placées les unes à còté des autres, qui constituent la paroi de la vésicule, et les cils qui mettent l'otolithe en mourement. A ce premier otolithe viennent, dans l'immense majorité des cas, s'en ajouter une quantité d'autres, généralement plus petits. La plupart des auteurs, surtout des auteurs plus récents, ont ru l'otoeyste se former d'abord, et pensent que l'otolithe naît par précipitation dans le liquide de la vésicule. Les observations qui font naître l'otolithe avant l'otocyste, peuvent bien être attribuées à l'insuffisance des moyens optiques arec lesquels elles ont été faites, l'otolithe étant, à l'origine, bien plus brillant et bien plus facile à voir utue la vésicule qui l'entoure. Les otocystes sont mu des premier's organes qui se montrent, lès le commencement de la période larvaire et dans la grande majorité des cas, longtemps arant l'apparition du système nerveux.

Frey (Lvus) a étudié l'ordre d'apparition des parties de l'organe auditif des Pulmonés et de Cyclas, mais ne donne aucun renseignement sur leur mode de formation. Togt (Lxir) les fail provenir, par crensement, d'une paire de boules opaques situées latéralement à la base dı pied d'Actceon, et qui descendraient elles-mèmes de la masse centrale de l'embryon. O. Schmidt (Lxxvs) a rit les oloeystes de Limax agr. se former peu de temps arant les ganglions nervenx, observation qui est confirmée par Gegenbaur (Lxxrur). Ge dernier auteur rapporte que l'otocyste de Limax est d'abord solide, puis se crense par écartement. L'otolithe se précipite dans le liquide de la résicule, dont les parois sont composées d'un épithélium vibratile. L’inégalité de déreloppement entre les eapsules auditives du còté droit et du côté gau- 
che, a été remarquée par J. Mïller (xcmi), che\% une larve de Pléropode gymnosome. Leydig avail déjà remarqué la même inégalité chez Paludina vivip. (rxxiv). L'otolithe n'est d'abord qu'un petit point, qui s'aceroît ensnite par ladjonetion de couches concentriques. Le mème auteur (cri) donne une très-bonne figure de l'otocyste de Cyclas. Sars, Koren et Damielssen (crx) remarquent que l'otolithe lapvaire de Buccimm se rompt, lorsqu'on le comprime. en quatre morceaux réguliers. Claparède (cxin) a observé que les otocystes apparaissent chez Neritima fluviat. comme vésieules, munies thacune t'un. canal qui se dirige ver's l'extérieur, et épaissies du côté où se tronve ce canal: re dernier disparaît plus lard. Faut-il considérer ce canal comme l'indice de la formation de l'otueyste par invagination de l'ectoderme chez cette espèce? C'est possible. Cet excellent ohservateur remarque que l'otolithe ne montre pas d'effervescence sous l'action des acides, mais qu'il se gonfle ot devient mou. Le gros otolithe embryonnaire lisparaît plus tard, lorsqu'il a été entouré d'une quantité d'otolithes plus petits. Chez Lymnapu, les capsules anditives ne se forment, d'après Lereboullet (cxxmu), qu’inprès les ganglions cérébraux et les yeux.

Ganine (cxxxix) déclare que les organes des sens, cliez les Pulmonés, proviemment de l'ectoderne. E. Ray Lankester (CXL) a ru l'oto cyste apparaître, comme vésicule pâle, inmédiatement au-dessous de la conche superticielle dont elle provient, dans son opinion, et déclare "rronée l'ilée que la résienle auditive puisse à son origine communiquer arec l'extérieur chez les Gastéropodes. Cette assertion absolue n'est rien moins que prouvée. Loin de lì, je ferai roir que l'olocyste de Limax' se forme par invagination de l'ectoderme. Langerhans (cхL) montre que l'otolithe larvaire apparaît, chez Acera, d'abord accolé à la paroi de l'otocyste, pour tomber ensuite dans la carité de cette résicule.

Les yeux ont étó trourés che\% la plupart des laryes de Céphalophores el de Lamellibranches; néanmoins leur mode de déreloppement est encore fort wbscur. Voici tons les renseignements que j'ai pu recueillir à ce sujet :

Jacquemin $(\mathrm{xL})$ a ru cet organe apparaitre, comme tache pigmentaire, à la base de chaque tentacule chez Planortis. Vau Beneden et Windisclimann font la mème observation chez Limar et ajoutent que la lentille apparait au milien du pigment. Alder et Hancock (Lı), Nordmann (LIII). Sars (L.TI) onl ru, rhez les firmmobranclies, les taches 
pigmentaires, rudiments des yeux, situées au-dessus et en arrière les otocystes. Lovén (Lxil) a décourert, chez sus larves de Lamellibranches marins, une paire d'yeux au-dessus des otocystes. Ce sont d'aborrl des vésicules renfermant quelques grains de pigment; plus tard, on aperçoit la lentille au milieu du pigment. Chez les larves de Teredo, les yeux, composés d'une lache do pigment arec un espace clair an centre, ont été vus par de Quatrefages (Lxxui).

Leydig (cxxiv) a vu l'oeil se former chez Palutina vivip. pen de temps après l'otoeyste. C'est une vésicule qui s'allonge en pointe en arrière. La paroi est composée d'une couche de petites cellules, qui donneront naissance à la sclérotique, la choroïde et la rétine. Le pigment se répose d’abord dans la partie postérieure de la paroi de la résicule, puis tout autour. La lentille apparaît comme point réfringent, renfermé dans une petite résicule, qui u’est probablement qu'une cellule élémentaire. Elle s'accroît ensuite par couches concentriques. Le corps vitré n’est que le liquide de la vésicule. Gegenbaur (xxxin) montre que, chez Limax agr., l'oil apparaît bientôt après l'otocyste ; e’est me masse cellulaire ovale logée dans le tentacule antérieur. Au milieu de eette masse se forme un corpusenle réfringent, renlermé, selon toute apparence, dans une cellule pleine de liquide. Lette lentille saccroìt par couches concentriques, au point de remplir complétement la vésicule oeulaire; il n'y a pas de corps vitré. La paroi de la résicule se différencie en deux eouches: la sclérotique et la cornée à l'extérieur, la choroïde à l'intérieur. Cette dernière se colore en rouge.

Chez Buccumu, les yeux se montrent, d'après Koren et Danielssen (Lxis), sous forme de vésicule située à la base des tentacules, ciliée intérieurement et renfermant de petits grains jaunes. Plus tard, les cils disparaissent, le pigment s'aceumule et la lentille se montre. Chez Purpura l'on roit parfois deux lentilles el deux taches pigmentaires par oril.

I. Mïller (LxxxıI) a signalé la présence des yeux chez les larves de Cleodora (Creseis) aciculata; Krohn (cxxv), ehez les larves de Cymbulia Peron., où ils se composent d'une lentille et d'un amas de pigment. Chez. Atlantu, l'ocil apparaîl, d'après Gegenbaur (ci), sous forme de vésicule, située en avant (au-dessus) de l'otocyste, auquel elle r'cssemble beaucoup. Cette résicule renferme, dans une cavité étroite, la grosse lentille sphérique. Au côté postérieur (inférieur) de la capsule, se trouve une masse cellulaire transparente. qui est probablement l'origine du 
bulhe. Ensuite la lentille grossit par l'apposition de couches concentriques; elle prend son origine dans le noyau d'une cellule, comme chez Limax. Le pigment lait encore défaut, et n'apparaîtra que plus tard. Chez Pterotrachera, e'est le pigment qui se montre le premier. Kỏohn (cхxy) a suivi le déreloppement des yeux chez Firoloüdes. Ce sont d'abord deux laches pigmentaires rondes, foncées, qui ne subissent pas de changements, pendant que l'wil se forme aulour d'elles. Elles se trouvent alors dans l’intérieur du bulbe, et disparaissent petit à petit. La lentille et le corps vitré paraissent se former en même temps. La rétine est striée perpendiculairement au corps vitré ; e’est la couche des bàtonnets. Elle prend ensuite une teinte rosée, qui tourne au brun chez l'adulte. Celte coloration n'a donc rien de commun avec le pigment, qui se forme, au premier abord, dans l'œil rudimentaire de la latre. Le déreloppement des yeux paraît être le même chez Pterolrachea.

Lereboullet (cxxvu) ne nous apprend rien sur le développement de l'œil du Lymnée, sinon que l'on voit apparaitre d’abord le pigment, ef ensuite le cristallin. Semper (cxxxis) montre que la résicule oculaire apparaîl, chez Ampullariu, avanl les tentacules. C'est une vésicule à parois épaisses composées de petites cellules et à contenu incolore. Le liquide de la résicule se condense pour former la lentille, qui devient réfringente dès qu'elle a acquis une certaine consistance. Le pigment apparaît petit à petit à la surface interne de la résicule.

D’après Salensky (cxxivis), les yeux se forment, chez Calyptrax sinensis et $N$ assa reticul., par invaginations de l'ecloderme, au bord antéricur du chanp entouré par le bourrelet voilier. L'entrée de l'invagination se resserre et se ferme; il n'en reste qu'un cordon, qui relie la vésicule oculaire à lectoderme, pendant qu'elle se porte à la rencontre des ganglions resophagiens. La cavité đle la vésicule est remplie de pigment. Stepanolf (cxxrvus) décril cinq taches pignentaires, munies chacune d'un rorps réfringent, qui se troureraient sur le voile de la larve déreloppéc de C'alyptrae et seraienl des yeux provisoires.

Langerhans (cxul) rapporte que, chez Areva bull., une des cellules de l'amats situé de chaque côté de l'osophage, el lestiné à former les yanglions, se détache et se divise, de laçon à produire $11 n$ petit amas distinct. Du pigment se montre au centre de ce deruier, el s'arrange sous la forme d'une lasse, dans laquelle la lentille se troure emboîtée. La lentille parait prorenir du noyau d'une cellule, et le pigment se déposerait dans la périphérie de la mème cellule. Le mème 
mode de formation de l'ail a été observé chez Ális. Chez Doris, se trouve, à la place de l'wil, un amas de cellules, dont chacunc renferme une gouttelette jaunâtre réfringente.

De toutes ces données il est permis de conclure que l'wil est d'abord une sphère cellulaire creuse, dont les parois se diflérencient pour former la rétine et peut-être aussi l'enveloppe du bulbe. Il est probable que la lentille se forme dans une des cellules des parois de la vésicule, quoique le tait ne soit pas suffisamment prouvé. Mais sur le premier développement de l'œil, sur les feuillets dont il lire son origine, el sur les procédés employés pour arriver à la formation de la vésicule primitive, les données manquent presque complétement.

Les tentacules supérieurs ou oculaires des Céphalophores se forment comme simples prolongements de la peau, dans le champ rirconscrit par le voile. L'ól vient généralement se placer à la base de ce tentacule, à son côté externe. Les Pulmonés terrestres, tels que Limax et Helix, font seuls exception à cette règle. Ici les trois tentacules forment d'abord une proéminence, située en avant du pied, à côté de la bouche, et se scindent ensuite en trois prolongements, dont le plus éloigné de la bouche devient le tentacule oculaire. Je n’ai pas besoin de citer les opinions des divers auteurs sur ce sujel; ils sont unanimes.

Les tentacules buctaux des Céphalophores se forment partout comme simples prolongements de la peau, dans la partie la plus roi sine de la bouche. Les appendices tentaculiformes de Deutalium, décrits par de Lacaze-Duthiers (cxiı), paraissent rentrer dans celte dernière catégorie.

Les otocystes, les reux et les tentacules d'un côté se forment souvent bien avant ceux du côté opposé.

LE SYSTĖME NERVEUX CENTRAL.

Laissant de còté les dounces nombreuses qui ne nous enseignent qu'une chose, le moment on tel ou tel observateur a commencé à discerner tel ou tel ganglion déjà parvenu à sa place délinitive, je ne rapporterai que les obserrations qui me paraissent réellement instructives.

Van Beneden et Windischmann (xLIV) ont vu, daus la partie antériemre du pied, une résicule, qui, à en juger par les figures, serait le ganglion pédieux, el yui donnerait naissance par différenciation à 
tout le collier asophagien. Lovén (Lxal) n̈indique aucun organe comme étant avee certitude l'origine l'un ganglion. Il n'émet à cet égard que de prudentes suppositions. Leydig (Lxxr) discerne, dans l'axe du pied de la larve, toute formée, de Paludina, un organe fusiforme jaunatre, le ganglion sous-osophagien. 0 . Schmidt (Lxxv) voit, ehez limax agrestis, les ganglions sous-cesophagiens apparaître, comme résicules jaunàtres, dans l'angle entre le pied et les lobes tentaculaires. L'observation est juste, seulenent l'auteur s'est trompé dans l'explication qu'il en donne; re sont les ganglions supérieurs qu'il a eus sous les yeux, ainsi que je le montrerai plus tard. Le même auteur rapporte que les deux petits ganglions du plexus splanchnique antérieur naissent sur les ganglions sous-asophagiens. Gegenbaur (Lxxviu) retronve fort bien l'origine du ganglion sous-asophagien dans la masse pyriforme jaune, qui se différencie dans l'épaisseur du pied. La masse sus-ásophagienne doit se former de son côté, mais n’a été distinguée que très-tard.

Sars, Koren ol Danielssen ( $\mathrm{xxx}$ et cIx) roient apparaitre chez Bucrimum les doux ganglions sus-aesophagiens el les deux ganglions pédienx. Les premiers se divisent rhaem en un gros ganglion qui en roie des nerfs aux yeux et au ganglion branchial, et un petit ganglion qui innerve l'otocyste et envoie une commissure au ganglion pédieux du mòme côté. Ce dernier innerve la branchie et le pied.

De Lacaze-Duthiers (cxil) montre que le ganglion pédienx de Dentalium apparaît au milieu du pied; e'est une masse rompacte, pyriforme, effilée à son extrémité antérieure (celle qui correspond à l'extrémité postérieure du ganglion pérlieux de Limax, Ref.), de couleur jaune-paille. Les ganglions sus-ussophagiens apparaissent de leur còté, plus tard que le ganglion du pied. Le même auteur (cxxin) dérrit l'apparition, chez Vermetus, de deux grosses taches opaques et jaunâtres, entre la base des tentacules, les yeux et l'œsophage; ce sont les ganglions rérébrö̈les. Lon distingue souvent deux autres taches entre l'ocil et le pied; ce sont sans doute les ganglions moyens. Une tache située dans le pied même, à sa base, tache jaunâtre et claire, parait ètre l’origine rles ganglions pédieur.

Lereboullet (cxxvu) rapporte que, chez des exemplatires de Lymnueus, peu avant l'éclosion, l'on roit deux amas de cellules rondes. grosses romparées an reste des rellules embryonnaires, munies de nucléi et de nucléoles, et situćes ì la nurque, où elles forment deux lobes symitriques. I e quinzième jour (en octobre), ces rellules se mul- 
tiplient et s'amoncellent sous la peau, dans l'espace compris entre le bord du manteau et les yeux, s'étendant jusque vers la base du pied et jusque dans l'intérieur des tentacules oculaires. Ces cellules formeront les ganglions cérébroïdes. Plus loin, l'auteur nous dit qu'après l'éclosion l'amas nerveux est remplacé par des amas de granules et de vésicules jaunàtres, au milien desquels se trouvent deux paires de corps globuleux. La paire antérieure, plus petite, est celle des ganglions sus-œsophagiens; la paire postérieure, plus grosse, celle des ganglions inférieurs. Ces corps ne tardent pas à se montrer composés de cellules gauglionnaires caractéristiques. Seulement, les dinensions de chaque ganglion étant égales à celles de l'une des cellules primilives, l'auteur se demande si chaque ganglion ne proviendrait pas de l'une de ces cellules. En sorte que, si je comprends bien cette descrip. tion un peu compliquée, l'amas primitif de cellules occupant la nuque serait destiné à disparaitre en majeure partie, quatre de ces cellules seulement domnant naissance aux quatre ganglions.

Salensky (cxxxvu) décrit le mode de formation du ganglion pédieux de Calyptrexa. L'ectoderme de la région antérieure du pied s’épaissit et forme mu disque qui fait saillie à l’intérieur; ceei a lieu à un moment où les otocystes sont déjà parfaitement développés et renferment un otolithe. Ce disque épais s'avance vers l'intérieur, et touche à l'entoderme d'un côté, à l'ectoderme de l'autre. Il devient plus tard pyriforme et atteint l'cesophage. La formation d'autres masses nerveuses n'a pas été observée, et l'auteur ne dit pas clairement s'il considère ce ganglion comme l'origine du ganglion pédicux seulement. Ganine (cxxxıx) se borue à déclarer que, chez Cyclas, Lymnous, Planorbis et Physa, le système nerveux provient du feuillet externe. E. Ray Lankester (cxi) rapporte que, chez Aplysia, le ganglion susosophagien se développe comme épaississement de la couche cellulaire externe dans la région en avant le la bonche. Il envoie des branches en avant et en bas; le ganglion sous-osophagien paraît n’ètre qu'un lobe de ce ganglion supérieur. Langerhans (CxL1) dit seulement que les ganglions se différencient, chez Acera, Doris et Eolis, sur les côtés de l'cosophage, formant vers la fin de la vie embryonuaire un amas situé de chaque côté de cet organe, amas auquel viennent s̀ajouter des cellules libres. Un petit amas, situé à côté de l'anus, serait un ganglion nerveux périphérique, qui enverrait un nert' jusqu’à une cellule étoilée, située sur l'estonac.

Résumer des opinions aussi diversus, des observations aussi incom- 
plètes n'est pas chose aisée. C'est plutôt une affaire de tact qu'une tâche réellement scientifique. Pour moi, mon impression générale est que le système nerveux des Céphalophores se forme de deux côtés à la fois : le ganglion pédieux dans le pied, probablement par dédoublement de l'ectoderme; les ganglions sus-œesophagiens dans la réguion céphalique, probablement aussi aux dépens de l'ectoderme.

LES ORGANES GÉNITALX.

Les rares auteurs qui parlent du mode de formation de la glande hermaphroditique, se bornent à dire qu'elle se détache de la parlie postérieure du foie; ainsi font Eisig (cxxxi) et Semper (cxxxir). Ganine (cxxхx) dit que l'épithèle des organes génitaux se forme aux dépens du feuillet externe; celte phrase demanderail à être précisée.

J. Müller (cxux) voit apparaître chez Creseis ncic. un organe en forme de bouteille, situé à côté de l'intestin et du diverticule de l'estomac (s.ac nutritif, R.), et dont le sommet s'étend jusqu'à la naissance de ce diverficule. Cet organe est situé du côté opposé an cơur; il est bien distinct dı foie, et ne pent être que l'origine de la glande génitale. Il s'étend en arrière, se sépare en lobes parallèles et ne larle pas à montrer, dans son intérieur, des œufs bien reconnaissables. Resterait à saroir quel est cet organe en forme de bouteille, quelles sont ses relations, son origine. Ce sont des questions anxquelles la description du grand anatomiste ne répond pas.

A part quelques indications trop vagues et trop isolées pour qu'il soit possible de les utiliser, le seul travail qui existe sur la formation les organes génitanx accessoires est relui de H. Eisig (cxxxı) relatif à diverses espèces du genre Lymmceus. Je ne puis que renvoyer le lecteur, qui désire avoir des détails, ì ce travail très-précis et très-soigné, et me bornerai à donner ici une courte amalyse des résultats principarx. Cies organes se forment après l’éclosion, et plus tard que la glande hermaphroditique. Ils apparaissent sous forme d'amas celluleux, poussant de la peau vers l’intérieur. Les organes mâles apparaissent les premiers sous forme de massue, dont le boui mince est attaché à la paroi du eorps, du côté droit, vers le milieu de la longuenr de la région antérieure. Celte massue se différencie d’abord en une enveloppe conjonctive et une partie centrale cellulaire et compacte. L’extrémité interne rle cette dernière se sépare par étranglement ef deviendra la prostate; la partie périphérique deviendra le pénis et le 
canal déférent. Elle s'étire à sou extrémité interne en forme de lasme batavique, el produit ainsi un cordon, d'abord solisle, qui se creuse par écartement et deriendra le canal déférent. Ce cordon est recourbé. et son extrémité se trouve an milieu de la musculature cutanée. Lal partie compacte du corps cellulaire se crense d'abord d'une ravité située près de la peau, le fourreau; sa partie interne donne naissance au pénis, qui se creuse d’un canal, rontinu arec celui du vase déférent. Des fibres musculaires apparaissent dans l'enveloppe de ces organes. Les organes femelles se montrent plus tarl que les précédents, sous forme de eylindre cellulaire solide, atlaché également à l’épiderme. Il sallonge et se renfle au bout interne, qui deviendra la glande à albumine, tandis que la partie externe ou allongée se fend en longueur en deux branches, qui sont réunies dans le roisinage de la glande albumineuse. La grosse branche reste unie à la peau et deviendra l'oviducte tout entier, avec tous ses élargissements, ses poches, etc. La branche inince part du sommet de la branche précédente et redescend dans la musculature cutanée, où elle rencontrera le canal déférent pour se souder arec lui. Cette ébauche des organes femelles se différencie de la même manière que celle des organes mâles; sa conche périphérique donne naissance ì l'enveloppe conjonctive et musculaire, sa partie centrale se creuse par écartement. Toviducte et le canal déférent n’entrent en communication arec la glande génitale proprement dite qu'au moment où celle-ci est déjà tı’ès-déreloppée et remplie de spermatozoaires mùrs.

11 semble résulter clairement de ces données que les organes génitaux accessoires et efférents se forment par prolifération et enfoncement des éléments de l’ecfoderme. Liorigine de la glande génitale est encore obscure.

Nous arons passé en rerue tout, ou à peu près tout ce que la bibliographie renferme de renseignements sur le développement des Lamellibranches, des Solénoconques et des Céphalophores. Arant de passer anx résultats de mes propres recherches, quil me soit permis d'attirer l'attention du lecteur sur les ressemblances nombreuses que présente ce développement aver celni des Rotifères. Analỹser les données relatives à ces animaux serait peut-être sortir du cadre du présent travail. Je me borne donc à signaler ce rapprochement et à attirer surtout l'attention sur le mémoire de Salensky, qui traite du déreloppement de Brachionus (Zeitschr. für uiss. Zool., vol. XXII, p. 4วั3, pl. XXXVIII). 


\section{INIEX BIBLIOGRAPIIOUL.}

1. Anistote. - Histoire des animaux. Les parlies des animaux. La ginération.

I1. G. Selducs. - Mistoria nalualis Teredinis, seu Xylophagi marini, in-40, 4 planches; 1733.

I!I. J.-J. Sivammerdan. - Bibel der Nalur, p. 53̈-83; 1737.

IV. Anonyme. - Einige Bemerkungen wegen kieiner Wasserschneclien (Hannover. Iagaz., 1. 801-822; 1763).

V. Bommé. - Sur le développement de Doris pilosa (Acla Soc. Flessing., t. III; 1773. V1. P. Forskid. - Descript. animal. qua in itin. orientali observ., in-40; 1775.

VII. J.-E-J. WaLcu. - Beitrag zur Zeugungsgesch. der Conchylien. Naturforscher, Stück XII, p. 1-52, pl. I; 1778.

VIII. A. DE LeEuvenhoek. - Arcana nat., t. II, leltre 95 et sniv.: 1793-97.

1X. J. Rsтпке. - Om Dammuslingen (Anodonta analina\}, avec 3 planches (Skrivt, nalurhist. selsk. Kiöbenh., I. IV. Ire liv., P. 139-179, et Q4 liv., 1) 173-18j; 1797 et 1798).

X. S.-F. Strnebl. - Ueber die Enhwick. der Teichhornschnechen Meckel's Archiv, t. I, p. $\{23$, et 1.11, p. $357 ; 1815$ et 1816$)$.

I1. G. Cinus. - Von d. aiiss. Lebensbeding d. weiss und kall-bhiligen Thiere, in $-4^{\circ}$ avee 2 planches, 1). 51-71; 1824.

.ilI. J.-L. PRÉvost. - Nole sur la géneralion des Moulelles (Ann. sc. nat., t. V, p. 323-4; 1823).

XIII. T.-L. Prévost. - De la génération chez la Moule des peinlres (Mém. Soc. phys. el hist. nat. de Genève, I. III, 1. 121-131, pl. I; 1823). - Reprorlnit dans .Inn. sc. nat., t. VII, 1. 447-4:3̈; 1826.

IIV. J.-F. Eschscmontz. - Bericht üb. d. zool. Ausbeute, ete. (Isis. p. 73з, pl. I, fig. $4 ; 182: 3)$.

XV. C.-1i. Cints. - Nene Beobachl. üb. d. Drehen d. Embryo im Ei d. Schnecken (Nov. Act. Acad. Leop. Carol., t. XIII, p. 763-772, pl. XXXIV; 1897).

XVI. C. Pfejffer. - Nalurgesch, deulscher Land. u. Susswasser Mollusken, 3 volumes aree ㄴ't planches; 1821-1828.

XVII. L.-L. Jacobsox. - Bidr. lil Blöddyren. Analomie og Physiol., 1. 1, in-ło. Kiöbenhavn; 1828.

IVIII. R.-Li. Lin.nT. - On the exist. and uses of cilia in the young of Gasterop. Holl. (Edinb. Journ. of science, t. VII, p. 121-125; 1827). - Reproduit daus Fror. Noliz., t. XVIIl, p. 303-309; 1827.

XIX. M.-H. DE Blanville. - liapport su un mémoire de Jacobson, fait a l'Académie le 24 décembre 1827. -- Reproduit ılans Ann. sc.nat., 1. XIV, p. 22-62;1828.

X.'. C. Préyost. - De la génération chez le Lymré (Mém. Soc. phys. el hist. nat. de Genève, t. 15: p. 197-208, arec planclies; 1828). - Reproduit dans Ann. sc. nat., t. XXX, 33-42; 1833 .

XX1. C.-1. Carus. - Niue Untersuch. ub. d. Enlwick. unserer Flussmuschel, in-40 arec 4 planches; 1892 (tiri des Verh. Leop.-Car. Akad., t. XVI, 1re partie).

XXII. A. Lund. - Recherches sur les enveloppes d'œu/s des Moll. gastérop., ete (Ann.sc.nal., 2e série, t. I, p. 84-112, pl. V1; 183i).

XXIII. A. DE Quatrefages. - Mémoire sur l'embryogénie des Planorbes el des Lymnées (Anı. sc. nal., 2e série, t. I1, p. 107-118; 1834).

XXIV. E. Jacudemx. - Vurläuf. Ber. Meiner Unters, ub. d. En/w. v. Planorb. $u$. Lymnaus (Isis, p. 337 344, pl. XHII; 1834). 
X.XV. H. Hen.D. - Ein Beitr.z. Gesch.d. Weichthiere (Ohen's /sis, 1). 998-1006; 153'). XXVI. C.-G. Canus. - Beitr. z. genauern Kenntniss d. Geschl.-Org. ein. Gaste?op. (Müller's Archiv, Jahrg. 1833, p. 487-199, pl. XII ; 1835 ).

XXVII. A. ve Quatrefages. - (Rapport de II. de Bedirvilee sur un mémoire de) Sur la vie inlrabranch. des petites Anodontes (. Acatímie des sc., 2 novembre 1830̈,.Reproduit dans Ann. sc. nat., 2e série, t. IV, 1. 283-299; 1833.

XXVIII. J.-L.-M. Launent. - Observalions sur le déveloupement des oufs de la Lunace grise et de la Limace rouge (Compt. rend. Acad. sc., 1). 228-230; 1835). - Reproduit dans ann. sc. nat., ge série, t. IV, p. 248-250; 1835.

XXIX. Li. WVhgNer. - Briefiche Mitheilungen (Arch. f. Nalurg. Jahrg., Il, Bd I, p. $369 ; 1836$.

XXX. A. DE Quatrefages. - Sur les petites Anodontes (Comples rend. Acad. sc., p. $294 ; 1836)$.

XXXI. J.-L.-M. Laurent. - Suite des observations sur le développement des Linaces et autres Moll. gastérop. (Compl, rend. Acad. sc., t. IV, p. 295-297; 1837, et l'Institut, V, no 198, 1. 62; 1837).

XXXII. J.-E. Gray. - On the enlargment of the eggs., etc. 'Charlesworth's magaz. of nat. hist., t. I, p. 247-288; 1837). - Heproduit dans Ann. sc. nal., 2e série, t. VII, p. $375-376 ; 1837$.

XXXIII. B.-C. Dumortiek. - Mémoire sur les évolut. de l'embr. dans les Hull. gastérup. (Nouv. Mém. Acad. Brux., t. X, avec 4 planches; 1837).

XXXIV. M. Sars. - Zur Entwicklungsgesch. d. Moll. u. Zooph. (Arch. f. Maturg., Jahrg. III, t. I, p. 402-404; 1837).

XXXV. M. DE SEmFEs. - Rech. sur l'anal. des .Holl. comparée à l'ovologie et a l'embryog. de l'homme et des Tertebrés (Compt. rend. Acad. $s c ., 1.17, \mathrm{p} .373-578 ; 1837$, et Ann. sc. nat., $2^{\text {e }}$ série, t. VIll, p. 168-174; 1837).

XXXVI. F. Dusarma. - Lettre sur les phénomènes présentés par des ceufs de Limace, etc. (Alln. sc. nut., 2* série, t. VIII, p. 374-5; 1837).

XXXVII. F.-A. Pouchet, - Sur le développ. de lembr. des Lymmées (Cumpt. rend. Acad.sc., L. VIl, p. 86-87; 1838 , et Ann. sc. nut., t. X, p. 63; 1838).

- Noies sur la structure du vitellus des Lymnées (Ann. franc. et étrang. d'onat., t. II, p. $233 ; 1838$ ).

XXXVIII. - J.-L.-M. Laurent. - Rech. sur le développ. des Limaces, elc. (Ann. franc. et étranc. danat., t. II, p. 132-157, avec planches; 1838).

XXXIX. M. Sars. - Entwicl. d. Gymnobranch. (Isis, p. 592; 1938).

XL. E. Jaceuenn. - Rech., etc, jer mém. contenant l'hist. du développ. du Planorbe (Nova Acl. Leop. Car. Acad., t. IVIII, ze partie, p. 635-678, pl. XLIX-LI ; 1838,

XLI. S.-L. Lovén. - Bidrag lit Känned. af Mollusk. Vitvekling (Vetensk. Aliad. Handlingar, p. 227-242, pl. II; Stockholm, 1839).-Reproduit dans Isis, p. 359-367; 1842 .

XLII. M.S.Ars. - Beitr. z. Entwicklungsgesch. d. Moll. u. Zooph. Arch. f. Natu'g., p. 196-219, pl. V et VI, 1. I; 1840).

Xlill. P.-J.vax Benenex. - Rech. sur le développ des Aplysies, Bull. Acad. krux., t. VII, p. 239-243̈, avec 1 planche; 1840). - Reproduit dlans Anń. sc. nat., 2e série, l. $\mathrm{XV}^{\dagger}$, p. 123-128, pl. I; 1841 .

XliV. P.-J. van Bexeden ot Cir. Wixdschnawx. - Mém. sur l'embryug. des Limaces, in-4o avec 1 planche; Brux., 18il.- Reprodnit dans Miuller's Arch., p. 176. 195 , pl. VIl et VIII; 1841.

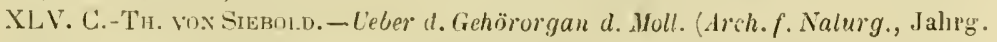
VII, Bil 1, P. 148; 18:1).

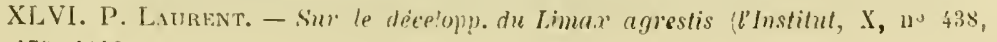
p. 179,1812 .

ARCII, DE \%OUL, EXP. ET GÉX. - T. 1Y. 1873. 
XLVII. 1. WOrbignx. - Vules sur des ceufs de Moll. recueillis en Patagonie (Ann. sc. nat, $2^{e}$ sírie, 1. XVII. p. 117-122; 1842).

XlVIII. 11. Ratnik. - Bomerk. Zib. d. Entsteh. einiger wirbellos. Thiere (Fror. Notiz., rol. XXIV, p. 161; 1842).

XLIX. A. PAAscil - Ueber d. Geschlechlssyst., elc., einiger Zwitterschnecken (Arch. f. Natmrg., Jahrg. 1X, vel. I, 11. 71-104, pl. V; 1843).

1. C.-P. HolbözL. - Bemaerlin. on Opbevar. af de lavere Südyr, etc. (Kröyer's Tidskrife, vol. IV, ]. $582-384 ; 1843$ ).

L.J. J. Aluer et A. IInNcock. - Devel. of Nudibr. (Ann. and Magaz. nal. hisl. vol. XII, p. 233̈; 1843). - Reproduit dans l'Institul, XII, p. 119; 18 '4.

LIl. Il. MEcked. - Ueber d. Geschlechlsapparat ein. hermaphrod. Thiere (Muller's Archiv, p. 484-503, pl. XIV et XV; 1844).

LIII. A. von Nordiann. - Versuch einer Monogr. a. Tergipes Edwardsii. Mém. présentés à l'Acad. de Saint-Pétersb., vol. IV, ؟. 495-602, pl. IV et V. - Traduction peu exacte dans Ann. sc. nal., t. V, 1846, p. 109-160, pl. I.

LIV. S. Lovén. - On nord. Hafs-Mollusker lOLfversigt Vetensk. Akad. Fürhandl., p. 49 à 53, pl. I, fig. 9 et $10 ; 1844 ;$.

LV. C. PEAcir. - On the Nidi of Purpura, ete. (Ann. and Mag. nat. hist., vol. XIII, p. $203 ; 1844)$.

LVI. G. Allian. - Cirrhopteron ('Institul, XII, 111; 1844).

LVII. M. Sars. - Zusälze zu d. Darst. d. Entwich. der Nudibranchien (Arch. f. Naturg., Jahrg. XI, p. 4-10, pl. I; 1845).

LVIII. H. Frex. - Ueber d. Entwick. d. Gehörwerk. d. Mollusk. (Arch. f. Naturg., Jalırg. XI, p. 217-222, pl. IX; 1845).

LIX. C.-Tir. von SizBold.- Handbuch der Zoolomie, 1re partie, Invertébrés, p. 240 et suiv., passim; 1845.

LX. G. AllmaN. - On the anat. of Actcon, etc. (Ann. a. Ilagaz.nat. hist., vol. XVI, p. $145 ; 1845)$.

LXI. C. Релсн. - On the devel. of Doris (Ann. a. Mag. nat. list., vol. XV, p. 445 $446 ; 1845)$.

LXIl. C. VoGt. - Rech. sur l'embryog. des Moll. gastérop. (Ann. sc. nat., $3^{\circledR}$ série, t. VI, p. 1-90, pl. 1-IV; 1846).

LXIII. A. Karsch. - Die Entwicklungsgesch. d. Lymnaus stagn., etc, (Arch. f. Naturg., Jahrg. XII, p. 236-27ṫ, pl. IX; 1846).

LXIV. J. REID.-On the devel. of the Nudibs. Moll. (Ann.a. Mag.nat.hist., vol. XVII, p. $377-389$, pl. X; 1846).

LXV. A. Kölliken. - Zur Lehre v. d. Furchungen (Arch. f. Naturg., Jahrg. XIII, vol. I, P. 9-22; 1847).

LXVI. F.-A. Pouciet. - Thérie posit. de l'ovulation sponianée, atlas in-40, pl. XVI et XVII; 1847.

LIVII. F. LeIDig. - Die Dotterfurchung, etc. (Isis, p. 177; 1848).

I.AVIII. I. Koren et D.-C. Danielssen. - Bemärk. til Mollusk. Idviking (Nyl. Mag. f. Naturvidensk., vol. V, p. 3; 1847). - Reproduit dans 1 sis, p. $202 ; 184$ S.

JXIX. Fr. Mugller. - Zur henntn. d. Furchungsproc. im Sclineckeneie (Arch. $f$. Valurg., Jalırg. XIV, vol. I, 1. 1-6; 1848).

LXX. H. Ratıк. - Zur Kennln. d. Furchungsm).im Schneckenpie (Arch.f. Naturg., Tahrg. XIV, vol. I, p. 157-162;1848).

LXX1. S.-L. Lovén. - Bidrag lil Käuned, on llıweckl. af Noll. Acephala Lamellibr. (Velensk. Akad. I!andl., p. 329-435, 1. X-XV; 1849). - Traduit dans Arch. f. . Vaturg., Jahrg. XT, vol. I, p. 312-339; 1849.

LXX11. MAx \$. Schultze. - Ueber d. Entwick, des Tergipes lacinulalus (Arch. \&. Naturg., Jalırg. XV, vol. 1, 1. 468-279, 11. V; 1849). 
LXXIII. 1. de Quatrefages. - Mém. sur l'emuryog. des Tarels (Am. se. nat, 3e série, t. Xl, p. 202-228, pl. IX; 1849).

LXXIV. F. LExDig. - Ueber Paludina vivipara (Zeilschr. f. w. Zool., vol. II, p. 127$130, \mathrm{pl}$. XI-XIII; 18300$)$.

LX.XV. N.-A. Wanneck. - Ueber d. Bild. n. Entwick. d. Embryos bei Gasterop. (Bullet. Soc. natural. de Moscon, t. XXIII, p. 90-194, pl. II-T; 1830).

LXXVI. O. Schmoт.- - Teb. d. Entwick. von Limax agrestis (Hüller's Archiv, p. $278-290$, pl. XII; 1851).

LXXVII. W. Biscir. - Beobacht. iib. Anal. n. Entw., ete., 1. 112. pl. VIII, lig. 10-12; Berlin, 1851.

LXXVIII. C. Geghnbaur. - Beitr. z. Entwicklungsyesch. der Landgaslerupoden (Zeitschr.f. w. Zool., vol. III, p. 371-4I1, pl. X, XI et XII; 18ü1).

LXXIX. C. Vogt. - Bilder aus dem Thierleben, 1 vol. ill-8o, 1. 289-296; 1832.

LXXX. J. Konex et D.-L. Daxielssex. - Bidrag til Pectinibr. Udvickl., in-so avec 4 planches; Bergen, $183 ̈ 1$ (supplément au même in-80; Bergen, 1852). - Traduit dans Ann. sc. nat., 3 e série, vol. XVIIIget XIX; 1832-33, ainsi que dans Arch. f. Naturg., 1853, et dans Ann. a. Mag. nat. hist., 1837.

L.XX1. L.-K. Scimard.1. - Einiges z. Entw, von Vermetus gigas (Denkschr. Wien. Akad., Bd IV, Abhandl. von Nichtmitgl., p. 133̈-137, pl. X; 1852).

L.XXII. J. Mueller. - Veber Synapta dig. u. ü. d. Erzeug. v. Schnecken in Holoth., in-folio, 10 planches; 18302.

LXXXIII. J. Mueller. - Ueb. d. Entwickelungsformen ein. niederen Thiere (10_ natsuer. Berl. Akad., p. 595-600; 1852).

LXXXIV. C. Gegenbaur. - Compt. rend. Acad. sc., p. $493 ; 1833$.

LXXXV. A. Hunbert. - Note sur les org. gén. des Pecten (Ann. sc. nat., 3e série, t. XX, p. $333-339 ; 1853$ ).

LXXXVI. A. Kronx. - Ueb. einen neuen mit Wimpersegeln vers. Gastrop. (Echinospira) (Arch. f. Nalurg., Jahrg. XIX, vol. I, p. 223-223̈; 18.33).

LXIXVII. A. Kölliker et C. Gegenbaur. - Entwich. von Pneumodermon, et C. Gegenbaur. Larve von Pneumodermon (Zeitschr. f. w. Zout., vol. IV, p. 333-334 et $369 ; 1833)$.

LXXXTIII. C. Davaine. - Rech. sur la génér. des llualres, 1 vol in-80 avec 2 plan ehes; Paris, 1853.

LXXXIX. G.-A.-F. Keber. - Leb. d. Eintrilt d. Samenzellen in das Ei, Insterburg, 1833.

AC. A. Mopun-Tandon. - Obs. sur trois Gasterop. orifnes (Journ. de conchyl., vol. IV, p. 223 -227; 1853 ;.

XCI. Th.-H. Huxley.-On the morphol. of the cephal. Hollnsca Philos. Transact.; 1853).

XCII. R. Leuckart. - Zoologische Untersuchungen, I. III. p. 64-67; 183 i.

XCIII. J. Mueller. - Ueb. versch. Formen v. Seethieren (Müller's drchiv. p. 69$74 ; 1834$ ).

XCIV. II. de LACAze-Duthiers. - Rech. sur les urg. génil. des Acéph. lamelihr.

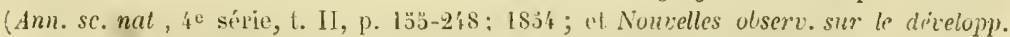
des fiutles (Comptes rendus Acad. sc., 1. XXXIX, p. 1197; 18.34, et l'Institul. t. XXIII, 1. $71 ; 185 \ddot{)})$.

XCV. O. Scmint. - Leb. d. Entwich. von Cyclas calyculata (Müller's Arch., 1. 428-438, pl. XVI; $135 i$ ).

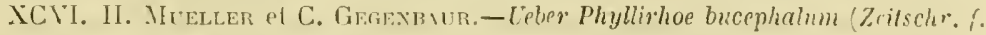
w. Zool., vol. V, p. $366 ; 183 \%)$.

XCVII. Tн. vox Inssuxg. - Einige Bemerk: z. Keber's duhandi, etc. (Zeischr. wiss. Zool., vol. XT, 1, 392-119, pl. XXI ; 18:\%). 
XCilll. L. Giegranbazr. - Bemerk, üb. d. Geschlechtsorij. v. Acteon (Zeischr. . w. Koot., vol. XV, 1. 436-441; 18:34).

fLIX. 11. Troscifet. - Beitr. z. Kennlniss d. Pteropoden (Arch. f. Nalurg., dahrg. XX, vol. 1, 1. 196-290, pl. VIII-X; 1834).

C. A. Mogun Tantox. - Histoire nat. des Mollusques, efe., 2 vol. ares allat, fer volume, p. 248 , pl. XXXVI; 183i-3̈.

Cl. L. Gegeniacr. - Untersuch. uib. Pleroporien u. Iteteropoden, 1 vol. ill-4; Leipzig, 1855.

CilI. F. LeYdik. - lieber Cyclas cornea (Niüler's Arch., p. 60-63, pl. VI; 1855).

CIII. G. Lindströas. - Bidr. Hil Känned. on OEstersj. inverl. Fauna (OEfvers, al' Velensk. Akad. Förhandl., p. 68-71, pl. 1II; 183̈5).

CIV. S. LovĖN. - Om Utveckt. hos slägt. Chiton (OEfvers. I etensk. Akad. Förhandl., p. 169-173, pl. IV; 1853). - Traduit dans Arch. f. Naturg., p. 206-210, pI. IX; 1836 , et dans Ann. a. Mlagaz. nat. hist., Yol. XVIl, p. 413-416; 1836.

CiV. A. Krohn. - Echinospira (Arch. f. Nalurg., Jahrg. XXl, vol. I, p. 1-5; 1855).

CVI. IV. Carpenten. - On the devel. of the emibr. of Purpura lapillus (Transact. Hicrusc. Soc., 2e série, vol. III, P. 17-30, avec 3 planches; 1855).

CVII. H. ue Lacaze-Duthiers. - Mem. sur le developp. des branchies des Moll. acéph. lamellibr. (Ann. sc. nal., $4^{\mathrm{e}}$ série, 1. V, p. s-46, pl. II ; 1856).

CVIII. C. VoGT - Beitr. zur Entwicklungsgesch. eines Cephaloph. (Zeitschr. f. w. Zool., vol. VII, p. 162-165, pl. X; 1856).

CiX. M. Sars, J. Koren et D.-C. DanielsSex. - Fauna littoralis Norvegice, vol. II, p. 25-46, pl. III-VI; Bergen, 1856.

LX. A. Linonx, - Beobacht. aus d. Entwichl. der Pleropoten u. Helerop. (Muller's Arch., p. 515-522; 1856, et ibid., P. 459-468; 1837).

CXI. O. Scnмnt. -- Zur Entwickl. der Najaden (I'ien. Sitzungsber. nalh.-nal. Cl., vol. AIx, p. $183 ; 1856)$.

CAII. II. de Lacaze-Duthers. - Histoire de l'organis. et du développ. du Dentale, ze partie (Ann. sc. nat., 4e série, I. VII, p. 171-255, pl. V-IX; 1857).

LXIII. R.-E. Ciraparède. - Analomie u. Entwichl. der Neritina fluvialitis (Muller's Archiv, p. 194-248, pl. Vil et VIII ; 1857).

CXIV. M. DE Sernes. - Des allérations que les Vollusques lamellibr. el gastérop. opèrent... sur tenrs coquilles (Ann. sc. nal., 4 e série, t. VII, p. 377-382; 1837).

GXV. C. Semper, - Beitr. zur Anat. u. Physiol. der Pulmon. (Zeitschr. f. w. Zool., vol. VIII, p. 381; 18̈37).

CXV'I. W. Carpenter. - On the devel. of Purpura (Ann. a. Mag. of nat. hist., 2e série, vol. XX, Г. 16-21; 1837, et lettre du docteur Dyster, abid., mème sujet; p. $127-128 ; 1857)$.

CXVII. O. Schmot. - Ueber... Najadeneier (Hien. Silzungsber. malh.-nat. Cl., vol. XẌll, p. 316i et $314 ; 1857)$.

CXVIII. A. Kronn. - Echinospira (Arch. f. Naturg., Jahrg. XXIll, p. 2ö2-261 ; 1857).

CXIX. J. MuelLek, - Bemerk, aus U. Entwickl. der Iteropoden (Honalsber. liert. Akad., p. 180-203; 1857).

CXX. A. Schneiner. - Vel. d. Enhwick. d. Phyilirhoe bureph. (Miller's Arch, p. 35-37, pl. III ; 1858.

CXXI. J.-D. MAcuoxal.D. - On the prob. metamorph. of Pedicularia, etc. (Transact. Linu. Soc., vol. XXII, 3e partie, p. 241-243, pl. XLII; 1858). - Comparez avec denx articles du même auleur, publit's précétemment, ì savoir: Remarles on the Anat. of

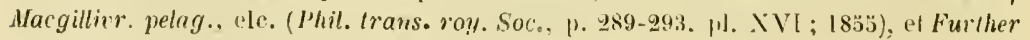
observ., ele. (ibid., 183̈3. 1. 295-297). 


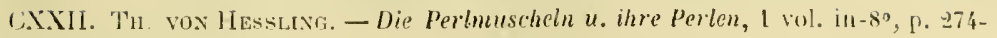
280 , pl. VII ; Leipzig, 1859.

CXXIII. H. de LAcaze-Dutmers. - Mém. sur l'anal. el l'embryog. des Vernurs, $2^{\mathrm{e}}$ partie (Ann. sc. nat., 4e série, t. XIII, 1). 266-296, pl. VII-IX; 1860).

CXXIV. A. Kroun. - Ueber d. Schale u. d. Larven d. Gasteropleron .Veck. (Arch. f. Naturg., Jahrg. XXVI, vol. I, p. 64-68, pl. II, fig. 2 et 3 ; 1860$\}$.

CXXV. 1. Kroun. - Beilr. zur Entwickl. der Pteropolen u. Heteropoten, $1 \mathrm{rol}$. in-40 avec 2 planches; Leipzig. 1860).

CXXVI. J.-D. Macdonald. - Furtherobserv. on the melamorph. of. Gastrop, etc. (Trans. Linn. Soc., vol. XXIII, $1^{\text {re }}$ partie, p. 69-81; 1860.

C.XXVII. IV. Keferstein et E. Enlers.-Beob. üb. d. Entwick. $v$. Aolis peregr., dans ies Zool. Beilv., 1 vol. in $4^{\circ}$, p. 98-100, pl. XV; 1861.

CXXVIII. A. Lereboultet.-Rech. d'embryol. comparée, ;e partie, Embryol. dı Lymnaus stagn. (Ann. sc. nat., 4e série, t. XVIII, p. 87-211, pl. XI-XIV Wis; 1862).

C.XIX. A. Stuart. - Ueb. d. Entwickl. einiger Opisthobr. (Zeitschrr. f. w. Zoo!., vol. XV, p. 94-102, pl. VII; 1865 ).

CXXX. H.-G. Broxx. - Die Klassen u. Ordnungen des Thierreichs, rol. III, $I^{\mathrm{r}}$ partie par Buoxn, $2^{\mathrm{e}}$ partie continuée par IV. Keferstein ; 1866.

CXXXI. H. Eisıg. - Beitr. z. Anal. u. Entwichl. der Geschlerhtsorg. von Lymnceus (Zeilschr.f. w. Zool., vol. XIX, p. 297-32l, pl. XXV; 1869;.

CXXXII. C. SEMPER. - Entwickl der Ampullarin polita, etc. (Natnurk. Verhandl. Utrechts Genoolsch., $1^{\text {re }}$ partie, ge livr., in-40 avec 4 planches; 1S62).

CXXXIII. G.-A.-F. KeBen. - Zur Controv. üb. d. Befruchl. d. Flussmuscheleies (Reichert's Arch., p. 284; 1869).

CXXXIV. H. DE LACAzE-Duthiers. - Eludes sur la morphol. des Mollusques (Compt. rend. Acad. sc., p. 1344; 1869 , et p. 43-46 ut 102-103; 1870).

CXX.T. E. Selenka. - Enhwick. von Tergipes claviger, ire parlie (Niedert. Arch. f. Zool., vol. I, p. 1-10, pI. I et II ; is71).

CXXXVI. E. Selenka. - Die Aulage d. Keimbl. bei Purpura lapillus (Niederl. Arch. f. Zool., vol. I, p. 211-218, pl. XVII ; 1872).

CXIXVII. IV. Salensky. - Beitr. zur Entwiclil. d. Prosobr. (Zeitschr. f. $w$. Zool., vol. XXII, p. 428-434, pl. XXXV-XXXVII; 1872).

CXXXVIII. P. Stepanofr. - Ueber d. Entwick, von Calyptrea (Bull. Soc. nalural. Moscou, vol. I, p. 115-123; 1873). - Extrait par l'auteur d'un mémoire publió précédemment en russe.

CXXXIX. M. Gaxine. - Beitr. z.Lehre v. d. embryon. Blällern b. d. Mollistien (Warschaner Univers. Berichle, $1^{\circ}$ 1, p. 115-171; 1873, ot Zeitschr. f. wiss. Zool.. vol. XXII, p. 285 ; 1872 ).

CXL. E. RAY Lankesten. - Stmmary of zoot. observ., de. Amn. a. Mag. nal. hist., $4^{\circ}$ série, rol. Xl, p. 83-87; 1873 .

CXLI. P. Langennans. - Zur Entwichl. der Gastroj. Opishobr. (Zeilschr. F. w. Zool., vol. XXIII, p. 171-179, 1)!. VIII; 1873,.

CXLII. W. Flewmixa. - Eeber d. ersien Ehtwichlerschcin. an Ei der Teichmu. schet (Arch. f. mikrosk. Anal., vol. X. p. 257-292, pl. XYl; 1874).

CXLIII. IV. Silexsky. - Bemerkungen uber Hachel's Fastra-Theorie (Arch. i. valurg.. Jahrg. Al, Bal I, J. 149-J32 et 1fis, pl. V. fig. 1-3. 


\section{DU DÉVELOPPEMENT EMBRYONNAIRE DES P'TEROPODES.}

De l'wuf' et de son développerment jusqu'à la fécondation. - Avant d'aborder la description de cette première partie du déreloppement, il sera bon de nous entendre sur le sens précis des termes qui vont ètre employés. En effet, ces lemmes sont en usage depuis que l’on s'occupe d'embryogínie, of les divers atuteurs les on t, depuis lors, enployés dans des acceptions aussi diverses, anssi changeantes que les idées ou les théories qui se sont succédé dans la science.

(bne devons-nous entendre par le mot ouf? L'usage vulgaire el l'exemple de la plupart des auteurs m'engagent à appliquer ce terme à l'ensemble du vitellus, de l'albumen et de la membrane albuminouse, et cela, à cause de l’absence dans notre langue d'un autre terme qui puisse désigner tout cet ensemble. Je réserverai le terme plus scientifique, plus précis de vitellus pour désigner la partie essentielle, la partie vivante el active de l'œuf, celle que beaucoup d'auteurs appellent l'ouf proprement dit. Le vilellus n'est donc pas pour moi un amas de substance nutritive destinée à être absorbée par la substance réellement vivante; c'est, au contraire, tout cet ensemble plus ou moins complexe, mais histologiquement comparable à une cellule, que fournit la glande ovarieme.

Ce vitellus se compose de substances fort distincles el faciles à discerner à première vuc : la substance homogène, finement granulée,

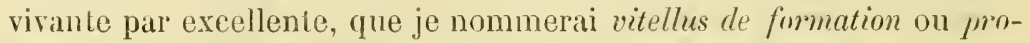
toplasme; et lạ substance réfringente, ì strueture globuleuse, le vitellus de mutrition. Mais il ne faudrail pas croire que ces deux termes servenl à désigner des parties du vitellus tout à fait distinctes lopographiquement. Tout au contraire, ces deux substances se pénètrent ch les globules nutritils sont enfermés dans un réséau de fils protoplasmiques, qui les mettent en mouvement et les font participer aux manilestations tle la vie. Ce n’est que chez les Géphalopodes que les deux substances se séparent d'une manière plus nette, la partie nutritive prenant un déreloppement considérable. Mais mème chez ces derniers il ne faudrail pas eroire à une séparation absolue; le protoplasme pénètre iei aussi plus on moins dans la masse de la substance nutri- 
tive et ne s’en sépare lout à lait, sous forme d’éléments histologiques distincts, que par la suite du dérelopjement.

Je nommerai indifféremment vésicule germinative on nucléus, le petit amas de substance particulièrement homogène, particulièrement transparente, souvent dépourvue de membrane propre, qui occupe le centre de la portion du vitellus où la substance formative domine. et qui disparaît et reparaît lors de la fécondation et à chaque segmentation, du moins chez les Ptéropodes et tous les Mollusques céphalophores que jai eu l'occasion d'observer.

Je n’ai que peu d'observations à rapporter sur le développement de l'œuf dans l'ovaire. L'oraire jeune est garni d'un épithèle simplę dont les cellules se multiplient par division. A un certain moment. celles de ces cellules qui sont restées accolées à la paroi de l’ovaire, se mettent à grossir beaucoup, leur protoplasme croissant beaucoup plus vite que leur nucléus. Ce dernier est très-risible, mais dépourru de nucléoles. Sur des ovaires plus arancés, le protoplasme des cellules a acquis des dimensions considérables et les glohules nutritifs se forment rlans son intérieur, par simple différenciation.

Lorule mùr est composé d'une membrane vitelline ou cellulaire, d'un amas périphérique de globules nutritifs, et d'un espace central occupé uniquement par du protoplasme, au milieu duquel se distingue le nucléus. Lat position relative des parties nutrilive et formative diffère done de celle que l'nn rencontre chez les vitellus fécondés, où res deux parties occupent deux moitiés de la sphère. lci elles sont concentriques, la partie nutritive enveloppant la partie formative. Cette dernière n'oreupe du reste plus tout à fait le centre chez des orules détachés de la glande. L'orule mûr est donc composé des mêmes parties essentielles que la cellule; c'est une cellule, et l'accumulation de substance nutritive dans son protoplasme ne peut lui ôter cette qualité. pas plus que l'aceumulation d'amidon dans une cellule végétale ne lui òte sa qualité de cellule. 
PREMIERE PÉRIDDE

Comprenant la période de segmentation depuis In fócondation. jusquà la formation des feuilles embryonnaires.

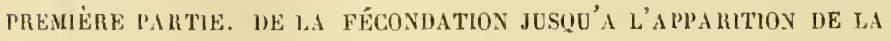
VÉSICULE GERMINATIVE.

Jai déjà parlé plus haul des diverses formes yu’atfectent les enveloppes des reufs, chez dirers Pléropodes. L'wuf lui-même se compose, chez tous ceux que j’ai pu observer, d'une membrane entièrement close, de tontes parts, élastique, résistante, à double contour, plus on moins orale on sphérique suivant les espèces, et aussi suivant les formes que prend le vitellus, par suite du fractionnement. Cette membrane renferme un albumen visqueux, parfaitement transparent et incolore, au milien dapuel se fonve snspendu le vitellus.

Je n’ai $p$ découvrir de membrane vilclline, après la ponte, chez ancun Pléropode; partont le vitellus était entièrement nu; mais j’incline à croire qu’il possìdo à la surface une consistance différente de relle qu’il a ians son intérieur, commel’a observé Warneck (voir p. 20).

La fécondation et les phénomònes qui doirent l'accompagner ont malheureusement échappé à mon observation. Tous les oufs pondus étaient déjì fécondés, et l'on royail, tant chans l’albumen de l’ouf que dans la substance albumineuse qui lenveloppe, des spermatozoaires immobiles. La membrane de l’œuf ne présente ancme onrerture et jamais on ne roit iei des spermatozoaires implantés dans m micropyle de eette membrane. La fécondation doit done aroir lieu avant que l'oul' s'entoure de sa membrane, et la présence des spermatozoaires à son extérieur indique simplement qu'une certaine quantité de sperme est descendne aver l'oul et s'est tromrée englobée avec lui dans les enveloppes albumineuses externes el internes.

Arant de procéder dans ma description, je dois olsserver que, la coustitulion de l'oul étant fort différente chez les deux groupes qui romposent la famille des Ptéropodes, jo me rerrai obligé de décrire ces deux trilus séparément. Je commencerai ì chaque stade par les Pléropodes thérosontes.

Pour olserver les premier's proesesus qui se produisent dans l'muf. sitot apros la ponte, je me suis adressí smrout à lespice la plus fa- 
rolable à ce genro d'observations, à la grante Cymbulia Promii. La fréquence des pontes, qui ont lieu à toute heure fle la journée, permet de saisir la chaine au moment mème où elle sort de l'utérus.

Le vitellus, an moment le lit ponte (pl. I, fig. 3, et pl. VIII, fig. 1). est dépourru de résirule germinative: Il comprend nue portion purement formative, unignement romposée d'un protoplasme finement ponctué $(\pi)$, et me portion constituéc 'n majeure partie de substance nutritive (on). La partie formative oceupe l'un des pôles du vitellus et assume une forme presque sphérique: C’est assez dire que la partie nutritive a me forme le ménisque ronvexo-concave, dont la concavité répond à la forme arrondie de la pirtie protoplasmique (p). VIIl, fig. 1).

Le protoplasme est, eomme je l'ai dit, linement grimulé, mais il ne faudrait pas en conclure que ces fins granules soient dispersés d'une manière quelennque dans le vitellus nourellement pondu. Tout au contraire, si l'on y regardr de près, à l'aide d'une bonne lentille à immersion, l'on saperçoit qu’ils sont arrangés d'une manière rég̨ulière, et cet arrangement devient très-frappant si l'on ajonte à la préparation une goutte d'acide acétigue. En effet, autour d'un centre, qui répond à peu près an centre de la partie protoplitsmique. l'on roit ces granules arrangés en ligness divergentes, formant une étoile d'une délicatessic el d'une finesse extrêmes. Le centre même n'est pas oceupé, comme l'on pourrait s'y aftendre, par un corpuscule différent du stroma qui l'entoure; non, ces mèmes granules qui composent l'étoile en oceupent aussi le milieu.

J'ai parlé de l'arrangement des granules : e'est quien effet c'est la senle partie visible, qui nous permette de nous rendre compte de l'arrangement intime de cette substance vivante, fui va, par son activité interne, derenir un etre semblable au parent. Nais loin de moi la pensée d’attribuer à ces granules une activité propre, de les considérer comme la partie la plus essentiellement vivante du protoplasme. Rien ne nous autorise à faire une telle supposition. Jusquà preuve du contraire, j’almettai que ces granules ne sont que des difiérenciations, dont la natme nous échappe, et qui no deviennent intéressiuts dans ce eas que parce qu’ils nous domnent une idée de ces monvements moléculaires intimes fui échappent à notre vue. Ce sont comme des jalons que cette substance, homogène pour nos morens optiques, entrainc arec elle et qui nous montrent qu'elle est active. el que son artivité est d'une nature parfaitement régulière et déterminée. 
La portion mulritive du vitellus se compose, comme nous l'arons vu, d'une nuasse de grobules fortement réfringents, homogènes et dépourvus de membrane propre, colorés de leintes qui varient du jaune clair au ronge orangé, suivant les espèces, el présentant chez quolyues-mues me struclure délerminée dans leur intérieur. Chez Cloudora pyramidata, chatue glohnle a une lorme polygonale due an rontacl avec les globules roisins, et montre dans son intérienr un noyau sphéricue (pl. Vll, lig. 3 el 9, $\left.g v^{\prime}\right)$. Le diamètre moyen des corpuscules étaut de 17 millièmes de millimètre, celui des noyaux est de 12 millièmes de millimètre. Cotle strueture rappelle satns donte relle de la cellule, mais la ressemblance n'est qu'apparente. I.e globule mutrilif ne jone jamais le rôle et n’atteint jamais le rangg d’élément hislologique. Chez Cavolinia tridentatu, les globules se montrent composés de plans superposés et alternativement plus et moins réfringents. Il résulte de là que lorsque le globule est tourné de telle facon que ces plans se présentent de profil, ils ont un aspect strié (pl. I, fig. 3 of $\left.4, g v^{\prime}\right)$ qui rappelle celui des museles striés. Lorsqu'au contraire le globule présente sass plans de face, il semble parfailement homogène ct dépourvu de loute texture intime. Il est facile de se convainere, par l'examen d'un embryon yui tourne, que chaque globule ne montre la strinre en question que lorsqüll se présente à l'œil dans un certain sens. La direction des plans varie d'un globule à l'autre. en solte fu'on ne roil jamais les stries que sur un petit nombre de globules ì la fois, et ces stries sont lirigées tantôt dans un sens, tantôt dans un antre, sans règle apparente (pl. I, fig. 3, $\left.g x^{\prime}\right)$. Somvent on discerne an milien des globes nutritifs quelques goutlelettes arrondies, réfringentes, de nature adipeuse (pl. VIII, fig: 1 et $:, g a$ ).

La ligne de démareation entre le protoplasme et la partie nutritive du ritellus est généralement irès-nette; mais il arrive parfois, par exemple chez Cymbulia Peronii, qu'une couche de proloplasme parsemé de pelits globules arrondis, réfringents el composés de la mème substance rue les globules beaneoup plus gros de la portion nutritive, vienne s'interposer entre ces deux parties du vitellus, étahlissant en quelque sorte la transition entre la substance purement formative et la substance presque uniquement nutritive (pl. VIII, fig. 1-8, grm). J'ai dit presque uniquement; e'est qu'en effet le vitellus de nutrition n’est jamais complétement dépourvu de proloplasme. Celui-ci pénètre dans les espaces que les globules laissent entre eux el y forme un stroma réticuló, les amas et des fỉs de sarcode, qui pénètrent dans 
tous les interstices el qu'une goutte d'acide acétique rend très-risibles (pl. I, fig. 4, $\pi r^{2}$. C'est sans doute à ce réseau protoplasmique qu’il faut attribuer les mowrements que l'on observe dans le vitellus de nutrition.

Tant que le vitellus de formation présente l'ćloile molćeulaire que nous avons décrile, le vitellus de nutrition obéil à la même altraction qui se manifeste dans le protoplasme, et l'on voit les globules arrangés nettement, surtout dans le voismage du vilellus de formation. suivant des lignes droites divergentes (pl. I, tig. 3 et i, gv), qui ont pour centre l'éloile protoplasmique. En mème temps, comme nous l'avons vu, la surface qui sert de limite aux deux portions de vitellus esl concave, la concavité étant tournée du còté de l'étoile.

$\mathrm{Au}$ bout de quelques minutes, l'étoile commence à s'allonger dans le sens du grand axe du vitellus. Bientôt elle se sépare en deux étoiles, dont l'une continue à oceuper le centre du protoplasme, tandis que l'autre atteint la surface au milieu de l'aire protoplasmique. L'on voit alors ce point se soulever comme un petit mamelon el puis se séparer entièrement, sous formé d’un globule sphérique (pl. I, fig. 3, et pl. VIII, lig. $1, x)$. C'est ce corpuscule qui a déjà été vu par tant d'observateurs et auquel on a attribué souveni une importance qu’il n'a pas. Diver's auteurs l'ont nommé corpuscule de direction (Richtungsbläschen, roy. p. 2.5). J'adopterai le terme de corpuscule excrété nu corpuscule de rebut, qui paraît plus conforme au rôle tout à fail nul qu'il joue dans la suite du développement. Il est expulsé du centre de l'aire proloplasmique, et comme ce centre est aussi celui des premiers phénomènes de segmentation, l'on en a conclu, bien à tor't, qu'il exerce une influence polaire.

Bientòt après sa sortie du vitellus, ce corpuscule se divise en deux moitiés généralement inégales. Jamais je n’ai vu deux corpusenles sortir sucessivement du vitellus, mais toujours un seul qui se divise ensuite. Ces deux corpuscules se différeneient dans leur intérieur et se montrent composés d'un nucléus et d'un protoplasme. Il sera encore question plus loin de ces formations, yui donnent à l'observatteur un point de repère, qui n’est pas à lédaigner, lorsqu’il cherche à s'orienter dans les starles successifs du développenent. Le point où ces globules sortent du vitellus deviendra le pòle formatif de l'axe embryogénique.

Pendant que les phénomènes que nous venons d'esquisser se passent hor's du vitellus, celui-ci n'est pas resté inactif. Nous avons vu 
l'éloile pui ocempait le milien du protoplisme se diviser an deux. L’une des étoiles s'est approchéc de la surlace et la parlie centrale de la substance est sortie du vilellus. Ce qui reste de cetle étoile périphérique se mòle pelil à pelil all reste dn protoplasme (pl. Vlll. fig. I (1 ュ, x'). L’aulre étoile, plus considérable, oceupe toujours le eentre du protoplasme. Son cenlre devient homogène et l'éloile disparaîl petil à petit (pl. VIII, lig. 1 el 2, \% el \%). Cetle disparilion se faif de la manière que j’ai le premier décrite chez l'iruf d’un Coelentéré'; au rentre de l'étoile apparail un corpuscule homogène, un pen moins réfringent que sum entonrage. Esit-ce me vésicule on mo copps plus ou moins solide? C"est dilfieile à dire. Bientôl apparaissenl deux on lrois aubres corpuscules on vósicules semblables, à côlé du premier. l'éloile s'uffare. les corpusenles se fusionnent pl. Vut, figg. 2, y, et il en résulte la résicule germinative on nucléus dlu vilellus lécondé.

Le vilellus des Gymmosomes diflère de celui des Thécosomes par une dillérentration moins nelte des parties mulrive et formative; le vilellus de mutrition se compose de globules plus pelits, qui arrivent presqur an conlact én nucléus el n’en sont séparés que par me conche proboplasmique très-mince. La quantibe de protoplasme dissémince entre les globules nulritifs est, en revanche, plus forte ane rhez les Thécosomes. La constitution du vitellus ressemble donc beaucoup à ce que j’ai observi chez les Hétéropodes.

DETXIẺUE PARTE. - DE L'APPARTTION DE LA VÉSTC1LE GERINATIVE IUSQU'A LA FORHE FRAMBOISÉE.

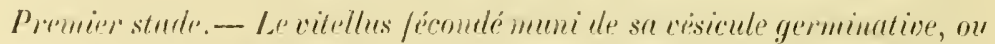
cellule de prentiere gézeration. - Nous en sommes venus alu point où l'on rencontre généralement les culs fraichement pondus, lorsquion n'a pas en le bonheur de ponvoir les recucillir an moment même où ils sortent de la vulve. Gest mu temps de repos, tpui peut se prolonger pendant me hemre ot davanlage, snivant la tempéralure. Après l’atIraction et la tension qui se manifostaient dans les éléments molérulairess rle la ecllule vifelline, pendant que l’élosile moléenlaire était

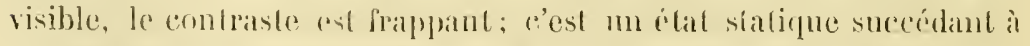

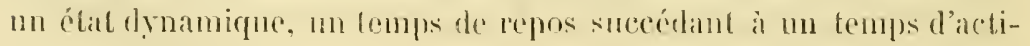

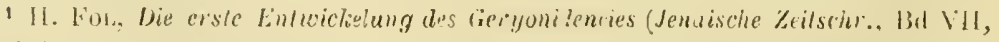
p. 㲅. 
vitó. C’est, en quelque sorte, mn moment de sommeil, pendant lequel les forces moléculaires se renomvellunt pour se manifoster bientôt à noureau. Le vitellus reprend une forme plus arroudie; la surfare de démarcation entre les deux parties du ritellus est plane, le protoplasme oceupant la petite moitic, le vitellus de untrition la plus grante moitió de lat collule vilelline (pl. VIll, fig. :3). Les granulations du protoplatsme semblent disséminces sans ortere; les globules mulritifs ne montrent plus ancune trace d'arrangement an liggnes droites. Ils sont placés suivant les règles qui président à la disposition de boules de grossenr's presque égales et qui se tonchent tontes.

Le nucléus, rui occupe tout le centre rlu protoplasme, est beaucoup plus homogène, et partant plus trasparent, que le protoplasme lui-mème; cependant sa substance nest pas si homogène, que l’on ne réussisse à y distinguer un pointillé très-fin. Quelles sont la composition chimique et la consistance de cette substance, et est-elle, ou non, entourée d'une membrane propre? Tout me porte à croire que c'est une substance homogène, plastique, moins fluide que le protoplasme envirommant. La présence d’une membrane propue de ce nucléus est facile à constater lorscru’on l'a altéré à l'ade de ríaclils chimiepues un yu’on l’a exposé au contact de l'eall de mer; mais je me garderais de eonclure de là à l’existence de cette membranc à l'étal vivant.

Le nucléole ou tache germinative mangue entièrement che\% tous les Ptéroporles que j’ai observés.

Les dimensions du nucléus sont énormes. comparées à celles du vitellus de formation (pl. Vili, tig. 3, v). Il mesure rhez Cymbulia, ò̀ il alleint proportionnellement les plus fortes dimensions, 37 millièmes de millinètre de diamètre, laudis que la partie protoplasmique

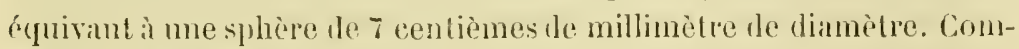
paré au vilellus tout entier, qui mesure 11 cenlièmes le millimèlre de diamètre, il paraitra moins disproportionné

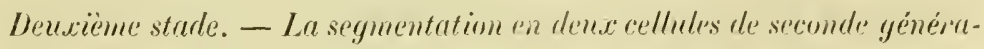
lion. - Apress un repos, qui varie d'un quat d'hemre à ume el mème plusieurs henres, suivant la tempiónature, le vilellus se prépare à la secmentation. Ce noyau lisparaît, il est remplacé par leux étoiles noléculaires qui, s'écartant l'une de l'autre, entrainent le vitellus dans deux

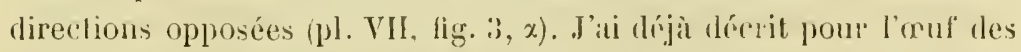
Géryonides ce plénomisne qu' personne n’a ru arant moi; mais. pour'

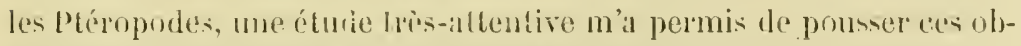
servalion- 
au moment où je savais que la vésicule germinative allail bientôt disparaître, j’ai vu deux poinls, dilfórant à peine de la subslance de la vésicule par un ponvoir de réfraction un peu diflérent, se nar(quer à la limile de la vésicule et du protoplasme, de denx côtŕs opposés, dans des directions qui répondent à celles où va bientôt aroir lien le lractionnement. De ces points partent bientôt des rayons droits divergents; l'apparence optique de ces rayous rappelle celle des stries que l'on woil dans un verre mal coulé (pl. VIII, lig. 4, x). Dès leur première apparition, ils se mettent à croître avee une grande rapidité, comme les cristaux qui se forment rlans une solution sursaturée. Le nombre des rayons va en augnieulant à mesnre qu'ils s'éloignent des centres. Bientôt les extrémilés de res rayons se rencontrent au milieu de la vésicule, et e'est à ce moment que celle-ci lisparaît. Je ne puis mienx comparer celle disparition qu'ì celle de ces images de lanterne magique, que l'on nomme dissolving vieu's. Il est ì noter que ces éloiles ne s'étendent qu’à l’intérieur du nucléus; le centre de chaque átoile se tronvant à la limite de celui-ci, il en résulte que l’on ne voit avant la disparition de la vésicule germinative que denx llemiítoiles qui se font face (pl. Vlll, fig. 4, x). Le proloplasme exlérieur an nucléus, même dans les points où il est presque en contact aveć le contre de l'étoile ( $\alpha$ ), ne montre aucun signe d'arrangement, du moins chez l'ouf vivant. Cet arrangement existe pourtant, el l'on peut le rendre manifeste par l'addition d'une goutte d'acide acétique. Mais, si l'on fail usage de ce réaclil au moment où le nucléus n’a yas encore disparu, il n’apparaîl anx yeux que deux étoiles moléculaires complìtes, pareilles à celles (pui se montrent lorsqu'on ne fait usage de ce réactif que quelques instants plus tard, au moment où la vésicule germinative a déjà disparu. L’on sail que l’acide acétique a généralement pour effel de rendre le nucléus plus distinet. Dans l'oruf des Géryonides, il fait apparaitre des restes de nucléus, à un noment où il n'est déjà plus visible chez l'ouf vivant, et où les éloiles se sont quelque peu écartécs. Ici, au contraire, ce même réactil fail disparaître le uncléus encore visible et ronne plus de netteté aux éloiles qui se sont montrées dans son intérieur. J'en conclus que le mucléus de l’nuf des fiéryonides a une couche superficielle plus condencée que le contenu, couche qui résiste à l’action de l'acide, Landis que le nucléus de l'muf des l'́éroporles est homog̨ène et plastique dans son entier.

lion romprendra que ces expériences si délidales, qui demandent 
tant de promptitude et d'attention el qui onl de plus pour résultat de tuer un assez grand nombre d'œufs et de conper court à toute observation ullérieure, n’ont pu ètre répétées que deux ou trois fois. En revanche, mes observalions sur les phémomènes qui se présentent sans l'emploi des 'réartifs ont été renourelées assez somrent pour donner à mes résultats un degré de certitude absolue.

Depuis la premic̀re apparition des centres d'altraction (car l'ubservation ultćrieure nous antorise à leur tomner ce nom dans l'intó. rienr du mucléns, jusquà la disparition de celui-ci, les modifieations se succòlent avec trop dr rapidité pour qu'on puisse les dessiner autrement que de mémoir's.

L’image qui sc présente ensuite est celle que j’ai dójà décrite chez les Géryonides: un protoplasme qui ne montre d'autres différenciations que ces deux étoiles de molécules, dont les rayons ront en se multipliant à mesure qu'ils s'éloignent des centres, et finissent par devenir confus vers la périphérie. La partie plus centrale de chaque éloile est facile à voir, sans l'aide d'aucun réactil (pl. VIl, fig. 3, a), mais l'acide acétique rend l'image hien plus nette et permet de suive le:s figures rayomées bien plus loin qu'on ne peut le faire sur l'nuf rivant (pl. VlII, fig. 5, ๙). Les rayons, situés dans la parlic précédemment occupée par le nucléus, vont souvent d'une étoile à l'autre en s'inlléchissant, en sorte que l'image rappelle vivement celle que l'on obtient en rersant de la limaille de fer sur une feuille de papier portée sur les extrémités d'un aimant en fer à cheral.

Sitôt après la disparition du nucléus, les centres d'atlraction se meltent à s'écarter lentement l'un de l'autre; puis, an bout d'un certain temps, il se produit à la surface de la portion protoplasmique, audessus du point où se trouvait la vésicule germinative, el conséquemment au-dessous de celui où se tronvent les corpuseules excrélés, m sillon perpendiculaire à la direction dans laquelle les étoiles cheminent. Avant même l'apparition de ce sillon, l'addition d'acide acétique fait apparaître une ligne de démarration très-nette entre les deux ćloiles, ligne formée par des gramules un peu plus gros que ceux du reste du protoplasme (pl. VIII, fig. 5, . .). Le sillon va en s'approfondissant et, en même temps, en s'allongeant, en sorte cqu'il linit par faire le tour de la portion nutrilive du vitellus. A ce moment le sillon est déjà tris-profond au point où il a commencó à apparâ̂tre, el l'on remarque yu’il n’est pas loul à fait perpendienlace à une ligne qui passerait par les deux centres d’attraction. Il tend, all conlraire, à faire 
avec catte ligne un anghle qui va en augmentant, comme l'a déjà décrit Watrnerk (roy. p. 29), mais sans atteindre cependant la granndeur de Sï degrés que Wanneck a obscrvée chez le Lymnée et la Linace. J'ajonterai, ce que cet antenr consciencienx a oublió de dire, quoique ses fignues l'ineliquent ofarement, yue ectle déviation a lien de droite à gauche, si l’on place le vitellus de telle façon que le nurléus el la partie protoplasmique soient tomrnós rers le bas, et si l'on suppose l'olsservateur occupant l'axe vertical du vitellus ainsi tourné. Ce n'est pas le fond du sillon qui subit la dévialion, c'est le bord de la cellule qui se trouve à gauche du sillon, qui le reconvre et s'élend mème jusque sur l'autre cellule (pl. Vlll, fig. 6). La séparation une fois complátée, les deux nouvelles sphères de segmentation se juxlaposent et ne présentent plus aucun caractère qui puisse rappeler l'obliquité (qu'a présentéc le plan de segmentation.

Pendant que les deux sphères rout en se séparant, elles ont une forme presque sphérique, en sorte que leur point de contact ra en diminuant d'élendue et que la projection de leurs contours finit par présenter la figure d’un is de chiffre. Mais, dès que la séparation est accomplie, elles se rapprochent à nouveau l'une de l'autre en s'aplalissant mutnellement.

C'est pendant que ce rapprochement s'opère, que réapparaissent les nucléi an centre de la partie protoplasmique de chaque cellule et de la manière que j’ai déjà décrite plus haut.

Le plan le démarcation entre les deux cellules est parfailement net; mais il no se produit pas iri, comme chez le Lynnée et la Limace, on comme chez les Géryonides, des vacuoles entre les cellules.

Lus denx cellules de scconde génération diffèrent beaucoup l'une de l'autle, chez les Pléroporles thécosomes, lant par leur grandeur que par leur composition. L’une des deux, plus petite d'un liers, est composće en majeure partie, on tont an moins pour la moitip de son volıme, de protoplasme, la partie nulritive formant la moitié ou lé tiers senlement de cette cellule. La grande cellule, au contraire, est rompuoséc presque miquement de matière untritive, la partie formalive ne représentant que le quart à peine de son volume lotal. Nous allons voir ces différences s'accentuel encore plus.

Lhez les Gymnosomes, ces deux collules sont pareilles. Icj aussi le uncléus disparail arant la division el ne reparaîl que lorsquelle est lerminée Mais, la partie protoplasmique élant à peine plus grrande que lo nuclíus arant a disparition, l'on pourait prendre celte partie 
protoplasmique pour le nucléus lui-mène. Cette erreur a élé effectivement commise par beaucoup d'auteurs, pour des reufs composés comme ceux des Gymmosomes (roy. p. 31). En regardant attentive ment, l'on retrouve ici les mèmes étoiles protoplasmiques que chez les Thécosomes. senlement elles sout plus petites et moins apparentes.

Troisième stade. - La segmentation en quatre cellules de troisiëme génération. - Les détails dans lesquels je suis entré sur les phénomènes intimes qui se prodıisent pendant le sccond stade, me dispensent d'en reparler ici. Chacume des deux cellules du stade précédent présente, en se divisant à son tour, la même série de changements : disparition de son nucléus, apparition de deux étoiles protoplasmiques (pl. 1, fig. 6, x), sillon qui devient oblique à la surface, séparation des nouvelles cellules, qui se rapprochent ensuite et se munissent chacme de son noyau. Seulement les quatre cellules qui résultent de cette seconde segmentation, diffèrent beaucoup l'une de l'autre. En effet, la grande cellıle du stade précédent s'est scindée en deux cellules, qui renferment toutes deux la même proportion de matière nutritive el formative que la cellule mère (pl. I, fig. $7, I, I I$ ); mais la plus petite des deux sphères de seconde génération a donné naissance à une petite cellule uniquement composée de protoplasme $(p l .1$, fig. $7, I V$ ) et à une cellule de la mème grandeur que les deux cellules provenant de la division de la grande sphère, et renfermant les deux substances dans les mêmes proportions que ces dernières (tig. $7, I I I$ ). Chez Cymbulia seule, l'on trouve dans la petite eellule un peu de substance nutritive, représentée par un certain nombre de ces grains réfringents qui étaient disséminés dans le protoplasme, à la limite entre les deux substances. Ces grains occupent, dans la petite cellule, la partie la plus éloignée des corpuscules d'excrétion, ou autrement dit la partie la plus roisine du pôle nutritif (pl. VIll, fig. $\mathrm{s}, I \mathrm{~V}, \mathrm{grn}$ ).

Ainsi done nous avons une ébauche embryonnaire composée de quatre cellules, dont trois sont à peu près égales de grandeur et de composition, renfermant une grande quantité de matière nutritive et peu de protoplasme, tandis que la quatrième est uniquement composée de matière formative. Je désignerai désormais les trois premières cellules sous le nom de cellules nutritives et la quatrième sous le nom de cellule formative de troisirme génération on du troisiène stade. Mais, tout en employant ces termes, qui ont l'arantage de la clarté, je tiens à faire une fois pour toutes cette réserve expresse que

- ARCh, De ZOOL. EXP. etT GÉN. - I. N. 18Tว. 
la cellule formative renferme thez certains genres quelque peu de sulstance nutrilive et que les edlules nutritives renlerment toujours une proportion considérable de protoplasme, el rue ce protoplasme forme même leur partic la plus essentielle, quoique la moins rolumineuse. En compatrat les ruantités de substance formative que renferme chacune des trois cellules nutritives, avec celle qui constitue la cellule proloplasmique, l’on reconnaît que ces fuantités ne sont pas lrès-inégales et que le vitellus des p'téropodes thécosomes, arrivé à ce point de développement, diffère de celui de la plupart des Céphatlophores surtout par l'adjonction de cette grosse masse nutritive qui est très-inégalement répartie entre les quatre cellules.

Les quatre cellules sont dans un mème plan; les trois cellules nutritives tournent leur partie formative, et par conséquent aussi leur nucléus, du côté où sont situés les corpuscules excrétés; dans la cellule formative, une vue de profil nous apprend que le nucléus n'occupe pas le centre de la cellule, mais se rapproche daranlage dı pôle formatif que du pôle nutritif (pl. V1II, fig. $8, I V$ ).

L'orientation de l'ébauche embryonnaire est dès maintenant indiquée. En effet, la cellule formative répond à l’extrémité postérieure de l’embryon (pl. I, lig. ‘, IV). La cellule nutritive, đlésignée sur la figure par le numéro 2, répond à l'extrémité céphalique de l'embryon. Je la nommerai désormais la cellule nutritive céphalique. Les deux autres cellules nutritives ( $I$ et $I I I$ ) oceupent les côtés; la plus grosset des trois $(I)$ étant très-probablement celle de gauche, la plus petite $(I I I)$, celle de droite. Enfin la ligne de rencontre des quatre cellules désigne un axe, qui répondra à l'axe larvaire. Ses deux extrémités peuvent déjà être distinguées; celle qui est désignée par la présence des corpuscules excrétés est le pôle formatif, l’autre le pôle nutritif (pl. VIII, fig. 8, Pf el $P n$ ). Il faut bien se garder de confondre le pôle formatif avee la cellule formative; celle dernière servira à former l'extrémité postérieure de l'embryon, celle qui se courrira de la coquille, tandis que le pôle formatif répond probablement au milieu de la face dorsale.

Chez les Gymnosomes, les quatre cellules de troisième génération sont égales entre elles et renferment lontes une forte proportion de substance nutritive.

Quatrième stude. - La segmentation en huit cellules. Le's cellules de quatrième génération. - Ce stade commence, comme les précédents, par la disparilion du nucléus el la formation d’étoiles moléenlateres: 
seulement la direction dans laquelle les centres d'attraction s'éloignent l'un de l'autre n’est plus horizontale : elle est verticale, e'està-dire parallèle à l'axe qui passe par les pôles formatif el nutritif ou parallèle à l'axe emloryogénique. Chaque cellule se divise done, mais elle se divise en deux moitiés tròs-inégales. La moitié inférieure, la plus rapprochée des corpuscules excrétés, est très-petite et uniquement composée de protoplasme, tandis que la moitié supérieure, beaucoup plus grosse, est, pour les cellules nutritives, composée en majeure partie de substance nutritive. Pour la cellule formative, la dilférence de taille tles leux moitiés est heaucoup moins marcuée et leur compusition est identique. En effet, les quatre nowvelles petites cellules qui résultent de ce fractionnement, sont parfaitement égales entre elles, ¿loù il résulte qu'elles représentent une perte beaucoup plus sensible pour la cellule formative que pour les trois cellules nutritives. Chez ces dernières, l'on n'aperçoil qu'une diminution dans la quantité de protoplasme, diminution qui ne tarde pas à disparaîtré, par le fait que le protoplasme se régénère chez res cellules aux dépens de la masse nutritive.

La différence de dimension des deux produits de cette segmentation est cause que l'observateur, qui s'en tiendrait à l'étude des formes extérienres, croirait avoir affaire ici à un hourgeonnement, plutôt qu’à un fractionnement.

La direction de la troisième segmentation est oblique, de même que les précédentes, d'où il résulte que les nouvelles cellules, au lieu de venir se placer au-dessous de chacune des grosses cellules, obliquent vers la giuche, l’observateur étant censé placé dans l'axe embryogénique, les pieds au pôle formatif, liı tête au pôle nutritif ${ }^{1}$, et se mettent dans l'angle compris entre la cellule ıni leur a donné naissance et celle qui y confine à gauche.

La rlisposition qui en résulte est représentée pl. I, tig. 8. Ces quatre petites cellules protoplasmiques $(1,2,3,4)$ forment le centre autour duquel, fomme nous le verrons plus loin, vientront s'ajouter

\footnotetext{
1 J'emploje ici, commo dans toute cettu période do développement, la manière de" lésigner la droite el la gauche qui est en usage ehez les mathématieiens, l'obser-

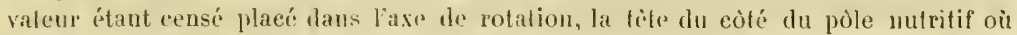
se formera la bouche. Pour boncoup de lecteurs, il pourrait etre plus rommode de se supposer placés ent dehors de l'axuf et le regardant de profil. Dans ce cas, il sulfira

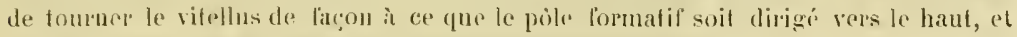
les désignations de lroite al dr ganche restemont les momes.
} 
d'autres cellules, qui formeront par leur ensemble le feuillet externe ou ectoderme. Le point où ces quatre cellules se touchent est done le pôle formatif $(\boldsymbol{P} f)$, lequel se trouve juste au-dessous fles corpuscules excrétés.

C'inquième stade. - La segmentation e'n douse cellules. Les cellules de cinquième génération (pl. l, fig. 9, $I^{\prime}, I I^{\prime}, I I I^{\prime}, I V^{\prime}$ ). - Une chose frappe déjà en lisant le titre du paragraphe, c'est que la progression du nombre des éléments histologiques cesse d'être géométrique et devient une progression arithmétique; l'on peut, si l'on veut, considérer ce genre de progression comme ayant commencé déjà au stade précédent, ainsi que le fait Warneck. Cette segmentation ne concerne, en effet, que les quatre grandes cellules de troisième génération, tandis que les cellules de quatrième génération n’y prennent aucune pari.

Par les procédés déjà suffisamment décrits, le protoplasme de chacune de ces quatre cellules se divise en deux parties inégales, dont la plus grosse conserve sa position au côté nutritif de l'ébauche enıbryonnaire, tandis que la plus petite vient se placer entre celle-ci et l'une des petites cellules de quatrième génération. Ces nouvelles cellules sont plus grosses que les petites du quatrième stade; elles se détachent obliquement des quatre grandes cellules, mais en se dirigeant vers la droite, tandis que les précédentes avaient obliqué à gauche (pl. l, lig. 9, $I^{\prime}, I I^{\prime}, I I I^{\prime}, I^{\prime}$ ). Et comme les nouvelles cellules s'inter. posent entre les petites et les grandes du stade précédent, il en résulte qu'elles soulèvent les quatre petites cellules et les font tourner en sens inverse de celui dans lequel celles-ci avaient d'abord obliqué (pl. l, fig. 9, $I, I I, I I I, I V$ ). Ainsi done, les cellules du cinquième stade se placent chacune dans l'angle entre la grosse cellule qui lui a donné naissance et la grosse cellule qui y confine à droite, et, en même temps, par ce móuvement, elles ramènent les petites cellules du quatrième stade, chacune au-dessous de la grosse cellule qui lui a donné naissance.

Sixième stade. - La segmentation en seize cellules. Les cellules de sixième génération (pl. I, fig. 10, et $\mathrm{pl}$. X, fig. 9, 1', 2', 3', 4'). - Les quatre petites cellules protoplasmiques du quatrième stade sont seules en cause cette fois-ci, loutes les autres restant immobiles. Les produits de la division sont encore inégaux, chacune des quatre cellules se divisant en une petile cellule qui vient se placer à droite et au-dessus de celle qui lui a donné naissance, dans les espaces libres que laissent entre elles les cellules du cinquième stade (pl. I, fig. 10, el pl. X, tig. I, 
I', 2', 3'. 4'), et en une rellule un peu plus grosse, qui continue à entourer le centre blastodermique, mais se trouve, par le fait de celte dernière segmentation, repoussée de nouveau vers la gauche, au-dessous des cellules du cinquième stade et dans la position quioccupaient dans l'origine les quatre cellules du quatrième stade (comparez pl. I, fig. 8, 9 et 10 , et pl. X, fig. $2,1,2,3,4$ ). Déjà, dans les segmentations précédentes, la division n'avait pas lieu pour les quatre cellules tout à fait en mème temps. Celte tendance s'accentue de plus en plus. Ainsi nous voyons sur la figure 9 que l'une des quatre cellules voisines du centre blastodermique (3) est déjà en voie de division, que son nu. cléus a disparu el qu'elle prend une forme allongée, tandis que les trois autres cellules de mème génération sont encore en repos.

Septième stade. - La division en vingt cellules. Les cellules de septième génération (pl. I, fig. 11, et pl. VIII, fig. 9, $\left.I^{\prime \prime}, I I^{\prime \prime}, I I I^{\prime \prime}, I V^{\prime \prime}\right)$. — Les quatre cellules $\left(I^{\prime}, I I^{\prime}, I I^{\prime}, I V^{\prime}\right)$ du cinquième stade se divisent seules (voyez fig. 11), ramenant de nouveau toutes les cellules, qui sont audessons d'elles, un peu rers la droite. Elles se divisent successivement, et la division de ce cycle est à peine terminée, que la segmentation suivante commence déjà. Bientôt l'on verra ces cellules, de générations différentes, se diviser en même temps, et ce défaut de ș̣nchronisme rend bien difficile, pour ne pas dire impossible, la tàche de l'observateur exact, qui cherche à analyser mathématiquement les phénomènes en apparence complexes qu'il étudie. Je ne pousserai done pas plus loin cette analyse pour le moment, me réservant de revenir sur ce sujet à propos d'autres Mollusques, ehez lesquéls on peut suirre pas à pas toutes les divisions jusqu ì la formation des deux premiers feuillets blastodermiques.

Chez les Gymnosomes (pl. X, fig. 2), toutes ces segmentations ont lieu exactement de la mème manière que chez les Thécosomes, à cette seule différence près, que les grosses cellules nutritives et les petites cellules formatives sont moins inégales de rlimensions, et que ces dernières renferment exactement les mêmes proportions de protoplasme ef de globules nutritif's que les premières. Cette substance nutritive, contenue dans les cellules formatires, ne larde pas à se dissoudre et à se changer en protoplasme, landis que celle des cellules nutritives subsiste beaucoup plus longtemps. 
SECUNDE PERIODE

Comprenant la période embryonnaire.

Première partie. - L'uchèvement du feuillet exterice au ectoderme. Depuis le rinquième stade jusqu'à présent, les quatre grosses sphères étaient restées inmobiles, les petites cellules du côté formatif se divisaient seules. Elles continuent encore à se diviser et à s'étendre, en formant une couche de plus en plus mince. Hais les grosses cellules rentrent en activité; en jetant un coup d'oil sur la figure 12, pl. II, qui représente un œuf arrivé au même point que celui de la figure 11 . pl. I, mais vu du côté opposé, el en la comparant arec les figures 14 et $1 \%$, pl. II, l'on s'aperçoit que la cellule protoplasmique $(I V)$ ste divise à sou tour. I.es trois cellules nutritives $(I, I I, I I I)$ en font autant. Jusqu'au huitième stade, la partie protoplasmique de ces cellules ćtait constamment tournée du côté du centre blastodermique. Elle change maintenant de place et se met à voyager tout autour de la cellule (royez pl. 11, lig. 13, 14. et 15, et pl. VIIJ, fig. 10, pr), surtout le long des lignes de contact de deux cellules voisines. A chaque étape, il se produit un temps d'arrêt, pendant lequel cette partie protoplasmique se divise, et il pésulte de chacune de ces divisions une petite cellule, situce à la surface (ji. 1I, fig. 13 et 15, et pl. VIII, fig. 10, ec), transparente, miquement composée de substance formative; en mème te̊mps, l'on observe une diminution à peine perceptible de la partie protoplasmique de la grosie cellule.

J'ai parlé de division; c’est qu'en effet le mode de formation de ces nouvelles cellules ne diffère en rien des divisions que nous avons déjà vues depuis le quatriène starle. Lês mêmes phénomènes se succèdent : disparition du nucléus, formation de deux centres d'attraction, puis séparation des deux masses et réapparition d'un nucléus dans chacune d'elles. Seulement. les deux éléments nouveaux sont excessivement inégaux de dimension et de composition, co qui, si l'on śen tient aux apparenees, pourrait lanire croire à un bourgeonnement plutôt quà̀ une véritable division d’éléments histologiques. s’il s'agissait seulement de rendre compte des changenents extérieur's et non de chercher à pénétrer leur véritable nature, le mot de bourgeomement serait plus juste. Toutefois, si l'on songe fue dans chaque grosse rellule cest la partie protoplasmique qui entre surtout 
en ligne de compte dans les manifestations d'activité vitale, les produits de ces divisions pilraîtront moins inégaux entre eux.

Après chaque division, le protoplasme des cellules nutritives paraît diminuer; néanmoins, nous le royons donner naissance, dans l'e pace de peu d'heures, à $u$ grand nombre de petites cellules protoplasmiques, doul la somme représente une duantité rle substanee formative bien supéricure à celle que ces grosses cellules renfermaient dans l'origine. C'est ru'en effet teur substance formative se régénère aux dépens de la substance nutritive, que nous royrins diminuer d'une manière assez sensible (eomparez les figures ò et 7, pl. I, aux figures 15 et 18. pl. Il ; toutes sont dessinées à la chambre claire d'Oberhäuser, sous un grossissement exactement le même de 15 o diamètres).

Le résultat de ces divisions est un envahissement progressif, mais irrégulier, de la surface par la couche ectodermique. L'enrahissement part du côté formatif (pl. II, fiğ. 13, et pl. VlII, fig. 10, Pf), et ne gagne que petit à petit le còté opposé (pl. II, fig. 14, 15̀ et 16), qui ne se recouvre que d’une manière très-irrégulière, en sorte qu il est difficile de dire au juste quel est le point des grosses cellules rui reste le plus longtemps ì découvert. la eouche extérieure, que jappellerai désornais l'ectoderme, présentant des laeunes, surtout chez Cavolinia. Mais toujours est-il que l'un des points qui restent le plus longLemps à découvert, est celui où les trois cellules nutritives se touchent an pôle nutrilif. L’on roit aussi que la partie protoplasmique des eellules nutritices, après avoir royagé tout le tom de l'embryon, finit pal arriver en ce point (pl. II, fig. 17, pl. V1I, fig. 4, et pl. IX, fig. 12, $\left.P_{n)}\right)$, où elle reste fort longtemps, donnant naissance, par le procédé suffisamment décrit plus haut, à quclques cellules ectodermiques, qui achèvent de clore le feuillet externe.

Ce point correspond très-probablement, comme nous allons le voir. an pôle oral de l'embryon. Que l'on prolonge la ligne sur laquelle les trois sphères nutritives se renenntrent, cette ligne coïneidera, à peu de chose près, avec l'axe embryogénique. D'un côté, il rencontrera la surface au point où va bientòt apparaître la bouche (pl. II, fig. 19, Pb), et de l'autre, il sortira par le milieu du dos, tantôt un peu plus près le l'extrémité inférieure, où apparaîtra la coquille (pl. II, fig. 19, Pb). tantôt un peu plus près du bout supérieur, qui va ètre entouré par le rnile.

L'nn remarquera fu’à l'origine les trois cellules nutritives oecu- 
paient trois angles d'un carré, dònt la cellute formative représentait le quatrième angle (pl. I, fig. 7). Cette dernière s'étant entièrement divisée en petites cellules qui se sont étalées à la surface, les denx sphérules nutritives latérales se sont rapprochées, de manière à former les deux angles inférienrs d'un triangle dont la cellule nutritive cóphalique forme l'angle supérieur (pl. II, fig. 17; pl. VII, fig. 4; pl. IX, fig. 13). Ces deux sphères latérales ou inférieures s'aplatissent mutuellement et tinissent par former, avec la troisième, un ovoüde plus ou moins arrondi ou allongé suivant les genres (pl. Il, tig. 18; pl. VII. fig. $5 ;$ pl. IX, fig. 1.ï), ovoüde dont le petit bout est formé par la cellule nutrilive céphalique recouverte par l'ecloderme, et le gros bout par les deux cellnles mutritives la térales, recouverfes aussi par l'ectoderme, qui est épaissi daus la région occupée précédemment par la cellule formative (mêmes figures, ece).

Chez Cleodora pyramidata, dont l'wul' a une forme allongée, l'embryon était, arant ces modifications, plus large que long, et se plaçait invariablement en travers, comme le montre la figure 4 (pl. VII). L'embryon, prenant maintenant une forme allongée, se trouve obligé de tomrner lentement dans la membrane de l'ouf pour venir se placer en long. La figure æ̈ (pl. VII) représente un ouf au moment où s’opère cette rotation.

Ce changement de position de l'embryon de Cleodor' pyramiduta n’est pas encore terminé, que déjà apparaissent de petits pinceanx de cils vibratiles, parsemés le long d'une zone circulaire qui deviendra le bourrelel voilier (pl. VII, fig. :, $v c$ ). Cette zone est rapprochée du petit bout de l'embryon et entoure, comme une couromne, la cellule nutritive que j’ai désignée sous le nom de céphalique. Cette dénomination se trouve done justifiće, et en même tempss il est démontré que la cellule formative et l'épaississement auquel elle dome naissance, se tronvent bien an pòle inférieur (comparer pl. VII, fig. 4, s̆, 6 et 7 ).

Mais maintenant se présente une difficulté bien plus sérieuse : celle de distinguer les côtés droil el gauche, les faces dursale el rentrale de l'embryon. Noublions pas que lat bouche se forme à l'un des points de rencontre des trois sphères mutritives. Or il n'y a que deux de ce's points de rencontre, l'un répondant an pôle formatif, l'autre an pôle nutritif. Il reste donc acquis el démontré que l'axe embryogénique coüncide avec l'axe qui relie le pôle oral au pôle aboral : que laxe longitudinal de la larve est perpendientaire à l'axe embryogéni- 
que. son pôle supérieur étant désigné par la cellule nutritive réphalique, son pòle inférieur par la cellule protoplasmique primitive. Mais il reste à savoir si la bouche se forme au pôle nutritif, ou si elle se forme au pòle diamétralement opposí:

Cette question pent paraittre singnlière aux persomes qui ne se sont pas beancoup occupées de díveloppements, et pourtant rien de plus facile que de commettre une erreur dans une détermination de re genre; nous ine serions pas embarrassé d'en eiter qui ont étí commises par les hommes les plus illustres dans thistrire de l'embryogénie.

Résoudre la fuestion par l'observation directe n'est pas chose aisée en ce qui concerne les Thécosomes orthoconques. En effet, les deux faces de l'embryon, la face ventrale et la face dorsale, deviennent si scmblables quil est très-difficile de les distinguer, l'épaississement. dı pôle formatif s'étalant et s'amincissant au point de ressembler parfaitement au pôle opposé. Suirre ces modifications pas à pas sous le microsenpe, en s'aidant de dessins faits à la chambre claire, n'est pas non plus facile, parce que l'embryon ne continue pas longtemps à se développer dans les conditions anumales où l'on est ohligé de le mettre pour l'observer, et aussi paree que l'apparition des cils motenr's, el la rolation qui en est la conséquence, à un moment où la position de la bonche n'est pas encore indiquéc, déjouent tous les efforts que l'on peut fairc pour ne pas perdre de vue l'orientation de l'embryon. Les Thécosomes à coquille enrouléc et les Gýmnosomes ne sont gunère plus farorables, pour d'autres raisons.

J'ai done cherché à tourner la difficulté en m'aidant des points de repère forluits que j’ai réussi à trouver, tels que la position des globules excrétés, ou cuelque irrégularité ou eorps étranger soit dans la membrane, soit dans l'albumen de l'cuf. J'ai cherché aussi à m’appuyer sur l'inégalité des denx sphères nutritives latérales pour arriver à distinguer la droite et la ganche de l'embryon.

C'est en employant ces méthodes que je suis arrivé à la conclusion que la bouche se développait au pôle formatif, et je suis revenu du bord de la mer persuadé qu'il én élait ainsi. Les raisons smr lesfuelles se basait cette opinion étaient les suivantes:

Les corpuscules excrélés se trouvaient, daus la plupart dies cas, sinon près du pôle oral, du moins sur celle des faces de l'embryon où se formait la bouche, c'est-à-dire sur la face opposće à celle nù se montrait déjà l’invagination préconchỵlienne (pl. I, fig. 18, x). 
La posilion des autres points de repère fortuits trouves dans labumen de l'oenf menail à la même conclusion.

Enfin, et ceri a surbul contribué à m’indnire en erreur, l'embryon de Cymlunlin (pl. IX, lig. 2) montre claisenent an pôle formatif me pelite fosselte (fig. $2, P n)$. Lat phus grande des trois cellules nuIrilives est licn à droite de la fignure, la plus pelite à gauche. Admettanl que celte fossette élait l'origine de la bouche, il en résultait clairenent que la bonche se développait au pôle nutrifif.

las résultats que j’ai ohtemus pour les Hétéropodes, el smblout pour les Gastéropodes pulmonós, ò il y a une invagination du pòle formatif, invagination qui persiste el devient la bouche, ainsi ([ue l'a déji montré Lereboullet, m’ont engagé à revoir attentivenent mes notes el mes dessins sur les Ptéropoles et à jeser les molifs sur lesquels s'appuyail ma première orientation.

Cen molifs me paraissent faibles et basés sur des eirconstances arcessoires on incertaines. Ainsi la position des eorpuscules excrélés est hien peu probante. Dans beaucoup de cas, ces coppuscules ont. disparu au moment où apparaît l’invaginalion préconchỵlienne. Dans d'autres, ils se trouvent du mème còté que celle invagination. Dans la plupart des eas, ils sont, il est rai, du côté opposé. Mais quelle preuve a-t-on que l'embryon n'ait pas tournó dans l'albumen pendant son développemint? Sous avons ru que, chez Cleodora, la forme de l'embryon et celle de la membrane de l'oruf le font changer de position. Chez les autres genres, je n’ai pas observé directement de rotalion avant l'apparition des eils vibraliles, mais il est possible qu'elle ait lien, causée par une diflérence de densilé daus les deux moitiés de l'embryon ou par toute aulre circonstance. Les corpuscules excrétés étanl adhérents à l'albumen ol non à l'embryon, cette supposition expliquerait les différences obserrées daus leur position romparé ì relle de l'embryon.

fluni qu'il en soil, les corpuseules le rebut sont un maurais point de repère yu’il vaut mieux laisser de côté. Chepehons à nous orienter silns leur secouls.

Les grosseurs divopses des cellules nutrilives el leur position dans l’intérieur de l'embryon donnent déjà de meillenres indications. Nais il faudrait hien se gauler de les prendre pour guides au delà du moment où elles commencent à se diviser, car les cellules nutritives qui résultent de lenr division ne conservent sourent pas les mèmes formes extérienres que les cellules mères (pl. 11, fig. 18), ou peurent 
même sarranger en masses disposées autrement et qui sont bien pro. pres à induire en erreur (comparer pl. II, figr. 17, 20 el 29l, m). Chez Crvolimin, ces cellules nutritives se divisent de bonne heure. en sorte quilil est impossible de s'aider le lemr situation pour poser l'embryon.

La formation d'une fossette au pôle formalif. chez Cymbulin, n’est pas douteuse (pl. IX, fig. 1;,$P f$ ), car ces fuits m’étaient déjà connus lorsque je cherchai, le printemps dernier, à dét(rminer la position de l'embryon, ot mes notes et mes dessins prouvent que les grandes sphères intérienres n’ont enrore subi aucun changement. Mais je me suis probablement trompé en prenant celle fossette pour l'origine de la bouche. Il semble, en effet, que, sur des embryons plus àgés, la houche, déjà reconnaissable (pl. IX, fig. 3, b), soit an pôle opposé à celui oì se forme la première petite fossette. En tout cas, les faits observés chez C'ymbulin ne soul pas assez clairs pour pouvoir servir de base à une détermination aussi importante.

Mais, si lexamen eritique de mes notes et de mes dessins ma montré le peru de fondement de mes premières conclusions, il ma heurensement aussi fourni des remarques qui me paraissent propres à trancher la question dans un autre sens. En effet, les figures 17, 18 , 20 et 2 I de la planche II montrent (toutes mesfigures étant rlessinées à la chambre claire arec la plus grande exactitude) que toute la région de l'ectoderme qui s'étend de l'un des pôles embry̧ogéniques (fig̣. 17, Pn) jusqu'en arrière du pòle inférieur, est épaissic d'une manière trèsmarquée. A liun des bouts de cet épaississement se forme l’inragination préconchỵliemne (fig. IS, qi); à l’antre bout, qui coïncide arec un pôle embryogénique, se forme la bouche (pl. 11, fig. 21, b). L’épaississement lui-même correspond donc au pied, mes dessins et mes notes sont d'accord là-dessus. Au moment où l'ectoderme achère de se refermer (pl. II. liğ. 17), celui des deux pôles embrỹgénirues qui forme la limite supéricure de l'épaississement paraît eorrespondre au pỏle nutritif, puisque r'est ici que se troure la partie protoplasmique des cellules nutrilives.

Chez Cleodurn myrnmidatu. lat chose est concore plus claire, car le pòle nutritif est parlaitement indiqué (pl. VII, figg. 4, ż et $6, P n)$ à un moment oì la position de l'épaississement du pied (éce) est déjà rertaine. La position des cellules mutritives, dont la plus grande est à droite, lat plus petite à tranche lorsque le pòle nutritif est tourué du rôté de l'observateur, cl cela à mu moment où ces cellules n'ont encore subi aucune division (comparer pl. VII, fig. 6. avec pl. II. fig. 12). 
prouve surabondamment la justrsse de cette orientation. Enfin le fait que la bouche se formera bien à ce pòle est prouvé par l'apparition hàtive de l'invacuination préconchylienne (pl. VII, fig. 7 , qi) à la partie inférieure de la face opposće à celle où se trouve le pôle nutritif (pl. VII, lig. 6, Pu).

Ainsi done, pour conclure, la roüncidence du pôle nutritif et du pôle oral me paraît hien établie. Je n’indique encore toutefois cette orientation que comme tròs-probable, me réservant de la vérifier à noureau sur nature à la prochaine oceasion.

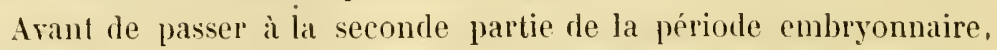
j'insisterai encore sur les désignations que j'emploierai par la suite pour indiquer les régions et les directions diverses de l'embryon. Je nomme axe embryogénique celui qui passe par les pôles nutritif et fornatif et plus tald par les pòles oral et aboral. Le pôle nutritif coïncide exactement avec le pôle oral, ainsi que le pôle formatif avec le pôle aboral. L'axe longitudinal passe par les centres de la cellule proloplasmique primitive et de la cellule formative qui lui fait face. Les deux extrómités de cet axe sunt le pôle céphalique ou supérieur et le pôle copuillier on inférieur. Ces deux axes forment entre eux d’abord un angle droit, qui va plus tart en diminuant, si bien que les deux axes se coufondent en un seul après la métamorphose. Ils détermineul un plan : le plan médian. Un plan perpendiculaire à celui-ci ef passant par l'axe longitudinal sera le plan latéral. La région qui entoure le pòle oral et s'étend de là vers le bas sera la région ventrale: la région dorsale entoure le pôle aboral; la région céphalique entoure le pòle céphalique.

Cette maniòre de placer l'animal diffère, je le sais, de celle qui est généralement en usage chez les auteur's qui traitent des Céphalophores. Ces auteurs placent le pied en bas, la bouche en avant, le dos en haut. Pour ma part, je ne rois aucune raison plausible de placer ainsi mes emhryons ; cette position est sans doute la position physiologique normale de beaucoup de Mollusques céphalophores, à l'élat adulte. (non pas de lous), mais elle a le désavantage de rendre difficile la comparaison aree des types d'antres embranchements, que l'ou a lhabitude de placer autrement. Je place douc mes animanx la tete en haut, la coquille en bas, la houche en avant et le dos cu arrière.

Seconde pantue. - L'apparition des premiers rudiments d'orgames. L'ordre dans lequel les nganes apparaissentsuccessivement chez les 
Mollusques n’a rien de lixe, comme on le sait déjà. Chez les Ptéropoles, cel ordre varie énormement; le voici tel qưil s'est présenté pour les différents genres que j’ai étuliés :

Chez Cacolinia, Hyalera et Hyalocylis : l'invagination préconchylienne, le pied, le roile, la bouche, les otocystes, la cavité stomacale;

Chez Cleodora et Styliola: le voile, l'invagination préconchylienne, les otocystes, la bouche, la cavité stomacale, le pied;

Chez Cymbulia Peronii el $C$. quadripunctata : la bouche, le pied, la cavité sfomacale, le voile, les otocystes, l'invagination préconchylienne ;

Chez Cliu : le voile, l'épaississement coquillier, la bouche, la cavité stomacale, les otocystes, le pied.

Cette variabilité, venant à l'appui des eas analogues que l'on connaît déjà, montre eumbien ees différences dans l’ordre d’apparition ont pen d'importance.

Le voile apparait sous forme d'une zone de cils, entourant comme une couronne l'extrémité antérieure de l'embryon. Cies cils sont d'abord très-fins et très-courts, présentant, dı reste, lous les caractères des cils vibratiles ordinaires, c’est-ì-dire qu’ils sont gros à la base et eflilés à la pointe; ils sont implantés par petites houppes, chacune sur le milieu de l'une des cellules qui sont destinées à oecuper plus tard le bord des disques motéurs, et qui, pour le moment, ne se distinguent en rien du reste des cellules ectodermiques (voir pl. VIl, lig. ॐ̆, et pl. X. lig. ॐ̆, vc). Lat disposition des houppes u’a rien de régrulier, si ce nest que toutes sout eontenues dans une cerlaine zone. Mais bientòt les cellules qui les porteut s'arrangent d'une manière régulière, en se multipliant, et forment un clouble rang, les cellules d'une rangée alternant en général arec celles de laautre (roir pl. 11, fig. 19,20 et 21 , el pl. Vll, fig. $7, \cdots / 1)$. Ces cellules ont léjà pris de l'épaisseur et forment um bourrelet peu accentué, comme on le voit en regardant le bord de la figure $\bar{\imath}$, pl. VIl. Les petites houppes s'élargissent et tendent à former deux rangées de cils.

L'on remarquera que la bouche se forme immédiatement an-dessous des rangées de cils, et qu'elle entame mène assez souvent le bourrelet voilier pl. II, fig. 19, et pl. Vl, fig. 1, b). Il y a, du reste, des variations sous ce ripport, et l'on voit combien les auteurs qui ont altaché lant d'importance a la position relative de la bouche el du roile, unt eu tor'l.

Dis que les cils apparaissent, l'embryon commence a se inouroir ; 
d'abord ce ne sont que des oseillations à peine perceptibles, puis des momrements de ra-el-rint, el, an moment on les houppes te eils se mellent sur deux rangs, l'embryour commenee à tourner.

11 est important de noter que ces cils sont les premier's qui se monIrent à la surface de l'embryou. Il n’y a pas chez les Ptéropodes de stade pendant lequel l'embryon se courre, comme relat paraît avoir lien chez d’autres Céphalophores (roir p. Zú el suir.), d’une toison de rils destinée à tomber avant l'apparition du voile. Jai passé des units entières au microscnpe pour ne pas perdre le fil du développement, ef je puis avancer, avee une parfaite assurance, que le state en question ne se présentr rhez ancun des lypes de Ptéroporles que j’ii éludiés.

Linvagimation méconchylienne apparaît au pôle aboral te l'embryon, c’est-à-dire à son extrémité postérienue el un peu du còté dorsal. Ciest d'aborl $n$ éprississement de la eoucht extodermique résultant de la profondem plus grande que les cellules de celte couche pussètent

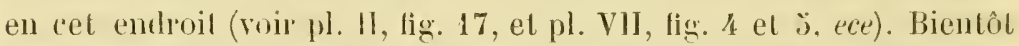
celle rógion commence à se renfoncer à son extrémité postrírieure el dorsale (pl. II, fig. 18, el pl. VII, fig. 7, qi). Il en résulte une cavité d'invagination en forme de gouttière, avec une ouverture élirée daus le sens longitudinal. Mais bientôt la cavitó s'apjorofondil, s'élargil el sun ouverture n’est plus qu'un fin canal à seetion circulaire (pl. VI, fig. I, qje). Co canal tébunche à la surface an milien d’une roselte parfailement régulière de cellules allongées, munies de grands uncléi (pl. II, fig. 22 el 23, el pl. Vl, lig. 1, qiv). Cetle invagination se trouve a l'extrémité infórieure de l'embryon, empićlant un peu sur la face dorsale. Ello ne so roit dune noltement que lorsqu'on regarde l'embryon par le côté dorsal (pl. VlI, fig. 7, el pl. II, fig. 20. qi) un bien de prolil.

La caritó d’invaginalion est spatciense et fail saillie à l’intérieur, repoussint devant elle les cellules nutritives. Les parois sont ́́paisses. el composées, de même que la roselte, de grandes cellules très-netles, nucléśs el nucléolées, formant une conche mique et préscntant un arrangenent des phus réguliers (pl. II, figg. 2 el 3, qi). Un coup d'reil sur la lignure 1, pl. Vl, domnera une idée des dimensions relalivement eousidérables que eelte invagination pent atteindre ì l'étal normal. Celle invagination, que j’ai retrouréc aussi che\% Limax, où elle persiste pendant tonte la vic. n’a, chez les Ptíroporles. fun'une existence éphé-

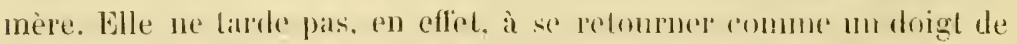


gant et à former une saillie plus ou moins conique ou arrondie; un coup d'wil sur les figures 22. 24, 20̀ el 19 de la planche 1l, qui représentent des étals sucressifs de l’invagination en train de se retomrner, donnera une idée asse\% rxacte du plocédé. Non-senlement la cavité se relourne, mais, en mème tempss, sus bords sont étirés el s'étalent, se confondant avee le reste de l'ectorlerme ; les parois de la cavité proprement dite font un pli du côté rentral en se retournant (fig. 2马̈, qs).

La sallie copuillière at me forme variable snivant les genres, mais constante dans chaque espèce. C'est sur elle, t’n effet, yu'apparaît aussitôl l'extrémitó de la corpuille, comme une culicule mince, dont la forme est exactement moulée snr celle de la saillie. Cette forme, qu’il est lacile de retrourer, même chez l’adulte, dans lous les genres qui gardent leur coquille embryonnaire, est très-caractéristique el me sera d’un grand secours dans la classification gue je proposerai du grompe des Orthoconçues, groupe jusuu’ì présent si embrouillé.

Lönvagination préeonchylienne atteint ses plus grandes dinensions dans le groupe des Thécosomes à coquille droile. Ghez C'lio, elle est très-petite et disparaît rapidement. Chez Cymbulia, elle n'est pas trèsrolumineuse, mais elle sécrète dans son intérieır des masses tulıerculeuse's foncées el très-réfringentes. Cette sécrétion a lien chez lous: les Pléropodes daus des cas anormanx; il suffit, pom l'obtenir, de tenip les rufs à une température basse. Ils se développent alor's lentement, et les conditions anormales dans lesquelles ils se trouvent, ont le temps d'agir sur enx et de provoquer des phénomènes pathologiques. Ces larves ont, dans ce cas, une carité préconchylienue trisgrande et remplie de grosses masses, el la coquille ne se forme pas. Il y a done denx alternatives : formation pathologique des masses internes avec ravité préconchylienne persistante, ou bien retommement normal de cette cavité avec formation d'une coquille externe et d'un mantealu. Les larves de Hyaléacées ohservées par C. Vogt (roir p. 69) montrent mème que si l'on mel iles larres, possédant déjà un conmencement de coquille, dans des conditions défavorables, elles perdent lem coquille, et l'invagination coquillière se reforme el se met à sérréter les masses dont j’ai parlé.

Chez C'ymbulia, la sécrélion de ces matsses paraît ètre le cas normal; en tout cas, je l'ai rue chez toules les larves que jai devées, el colle scórétion, pourvu qu’elle ne dépasse pas reptaines limites, n’empeoche en aucune fiacon la formation de la coquille. Il ny a pas reompnement

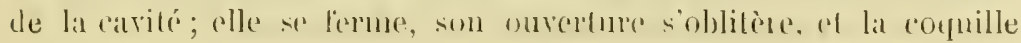


apparait en forme de verre de montre sur la portion de l'ectoderme qui entourait cette ouverture (pl. IX, lig. 16 et 18, q). Plus tard les parois cellulaires de l’invagination préconchylienne se résorbent et les masses tuberenleuses restent dans la tavité du corps, attachées aux lissus qui tapissent intérienrement la coquille (pl. IX, fig. 18 et 19, qim).

Guelle est la composition chimique de ces masses? Elle est assez complexe et paraît identique à celle du premier rudiment de la coquille. Si l'on traite par l'acide acélique, l'une el l'autre se ramollissent et premnent des contours plus arrondis, mais sans dégagement de gaz. Lacide chlorhydrique concentré provoque la formation de bulles gazenses, ce qui démontre la présence d'une certaine quautité de calcaire. La partie qui reste après ce traitement ne s'altìre plus par les acides et ne se gontle que tres-lentement dans les alcalis. C'est donc probablement une substance chitineuse on cornée.

La bouche, à sa première apparition, n'est qu'un petil ensemble de cellules ectodermiques arrangécs en rosette régulière (roir pl. 11, figg. 21, b). Bicutôt l’on discerne au milien de cette rosette un enfoncement en lorme d'entonnoir; la conche externe, épaissie en eet endroit, se renfoncant en dedans. Cela se voit surtout sur me rue de prolil (pl. II, fig. 19, b); de face, la bouche se présente en ce moment comme une petite lossette cntourée d'un bourrelet rond (pl. VII, tig. 10, b). La lossette, en sapprofondissant, devient un canal à parois épaisses, terminć en cul-de-sac et dirigé de haut en bas et de dehors en aledans (pl.VI, fig. I, $a^{\prime}$ ). Le bout lermé du tube scmble quelquetois un peu rentlé (pl. III, fig. 36, (o). Ce canal est de bonue heure lapissé de cils vibratiles. Il domnera naissance à l'nesophage el à ses appendices.

Lorsque le cacum cesophagien a déjà alteint une certaine profondeur, l'on remarque une saillie longitudinale (pl. VI, fig. 1, as) qui part du bord dorsal de la bouche et descend dans le tube osophagien, où elle se perd bientòt. Cette sitillie est formée d'un ensemble de cellules épithéliales un pen plus allongées que les autres. Elle redisparaît chez les P'ćropodes el mérite senlement d’ètre notée paré qu’elle se retronve chez les Gastéropodes pulmonós, où elle acquient me importance plus grande.

Le pred s'aceuse de fort bonne henre chez certains genres, par exemple Cacolmia, Hyuliea : chez Clio el Cleodora son apparition est si tardive, que l'on ne peut pas encore le distinguer nettement an moment où lá bonche et les otueystes sont lormés $(\mu$ l. VIl, tigg. 10, et 
pl. X, lig. 6). Chez les Hyaléacées, il apparaîl d'abord comme un épaississement de l'ectoderme. En regardant l'embryon par le côté ventral (pl. II, fig. 21, pe), l'on voil que la région épaissie a la forme d’un ovale ou même d’un losange. L'angle supérieur du losange est formé par la bouche (fig. 21, b) ; l'angle postérieur est formé par l'invagination préconchylienne, en avanl de larpuelle, du côté ventral, se trouvent deux cellules qui font saillie (fig. 21, ac, et pl. VI, fig. 1, ac) et qui marquent l'endroil où se formera l'anus. L'épaississement s'aecuse surtout sur les bords de la région du pied, formant deux bourrelets latéraux, comme deux croissants qui se toncheraient par lenrs pointes. En regardant l'embryon d'en haut (pl. II, fig. 17, pe), l'on voit de très-bomne heure les bơurrelets et l'épaississement, et l'on remarque que l'ectoderme est composé, surtout sur les bords, de cellules très-grandes. Mais, entre celte couche eetodermique et les cellules nutritives, l'on remarque une couche locale de cellules, qui proviennent peut-être de la division de la cellule formative primitive.

La partie médiane du pied commence alors à faire saillie en dehors (pl. II, fig. 19, p, et pl. VI, fig. 1, $\mu$ ); ses bords latéraux montrent toujours les mèmes grosses cellules (pl. VI, fig. 1, pe), et une couche interne ou mésodermique, aux dépens de laquelle nous verrons se former d'abord les olocystes, puis des muscles.

La cavité digestive de l'embryon se forme par une différenciation des cellules nutritives. Reprenons notre embryon au moment où les trois cellules riutritives sont entore entières (pl. II, fig. 17, et pl. VII, tig. 4). Les parties protoplasmiques de ces cellules sont tournées rers l'extérieur, ou plutôt vers l'ectodermc, à l'endroit où se formera la bouche. Bientôt après, ees parties formatires ont disparu de la surface, et, en examinant attentivement le centre de l'cuf, je les y retrouve; elles se sont done déplacées, comme elles n’ont cessé de le faire pendant toute la formation du feuillet externe, et, après avoir. pareouru toute la surface de l'embryon, elles sont renues se rencontrer à son centre. C'est à ce moment qu’a lieu, ehez les Hyaléacées, la division des trois grosses cellules. En examinant le centre de l'embryon, j’ai cru voir que chacun de ces segments de sphère avait un nucléus et une partie formative à son angle interne. Nous n'aulions done affaire ici qu’̀̀ une division de cellules, qui diffère des précédentes en ce que chacum des produits de la division emmène arec lui une moitié de la substance nutritive de la cellule mère. Mais à ce genre de division vient's'en ajonter un autre qui est pareil à ce- 
lui qui a eu lieu à la surface. Ces six cellules nutritives, ear tel est à peu près leur nombre maintenant, se divisent en une couche interne de cellules petites, uniquement eomposées de protoplasme, et en une couche externe de cellules composées en majeure partie de sulsstance nutritive. En effet, si nous traitons, en re moment, l'embryon par l'acide acétique (pl. II, fig. 20, en), nous voyons au centre, un peu plus rapproehé de la face ventrale que de la face clorsale, un petit amas de cellules dont les unes, périphériques, sont en continuité avec la substance nutritive, tandis que les autres, qui occupent le centre de l'amas, sont petites, arrondies et indépendantes. Ces cellules ront en se multipliant, et, par l'écartement qui se produit par' suite de l'agrandissement de l'embryon, elles forment les parois d'unc cavité ovale, la cavité digestive primitive.

Cherehons ì nous rendre compte au point de vue blastogénique des phénomènes que je viens de décrire. Le feuillet interne ou entoderme est constitué dès que l'ectoderme est formé et que les sphères centrales ont cessé de se diviser à leur surface. Ce feuillet interne est composé, il est vrai, seulement de trois cellules, monstrueuses par la prédominance de la matière nutritive qu'elles renferment sur leur partie vivante et active, leur protoplasme et leur nucléus. Mais ce n'en sont pas moins de véritables cellules. Il est clair que nous ne pouvons parler ici de feuillets qu'au'figuré; si l'on prend les choses à la lettre, trois cellules ne peuvent former un fenillét. Ces cellules se dirisent d'abord en largeur, puis en profondeur'; de même qu'à la surface, il y a ici une division inégale, ou, si l'on veut, un bourgeonnement. De ce bourgeonnement résulte une différenciation de l'entoderme en deux couches, dont l'une, interne, forme un véritable feuillet, tandis que le reste de l'entoderme conserve le même caractère qu'auparavant de cellules énormes, peu nombreuses, dans lesquelles le protoplasme disparaît presque au milieu de la substance nutritive. Nous verrons plus loin que la cavité centrale donne naissance à l'estomac el à l'intestin, tandis que les cellules nutritives formeront un sac, le sac nutritif. lequel reste en communication avec le tube intestinal.

Il est une question cependant que je me suis posée, mais que je n'ai pu réussir ì résoudre d'une manière satisfaisante: celle de savoir si la cellule formative primitive participe ì la formation de ce feuillet gastrique.

Chez les Thécosomes campyloconques, les cellules nutritives sont relativement un peu moins grosses et se divisent plus vite, formant une 
petite cavité interne au moment où le pôle nutritif vient à peine de se refermer. La bouche se forme de bonne heure (pl. IX, fig. $1 \mathrm{~J}$ et 17, b). Chez les Gymmosomes, les cellules nutritives sont encore moins grosies. Ici aussi, le feuillet gastrique se forme par un dédoublement des cellules nutritives, qui se sont multipliées par division, arant même la clôture complète de la couche ectodermique (pl. X, fig. 3, en). Ce mode de déreloppement établit la transition entre la formation des feuillets par une donble série de divisions des cellules nutritives chez les Ptéropodes thécosomes, el la formation de ces fenillets par une invagination bien accentuée, comme je l'ai observée chez. les Hétéropodes.

J'examinerai ce sujet plus en détail lorsque j'exposerai au lecteur le résultat de mes observations sur les Hétéropodes.

En examinant la moitié orale de la région céphàlique des embryons représentés pl. II, fig. 19, et pl. VI, fig. 1, l’on remarquera que cette région (ne) est épaissie, e'est-ì-dire composée de cellules plus fortes et plus épaisses que la moitié aborale du champ circonscrit par le voile. C'est de cet épaississement que nous verrons plus tard naître la masse nerveuse sus-œsophagienne. 


\section{LA PERIODE LARVARE DU DEVELOPPEMEN'T DES P'TÉROPODES.}

Nous avons passé en revue pendant la période précédente la formation des feuillets embryonnaires, puis l'apparition des premiers organes sous forme d'ébauches rudimentaires. Pour suivre le développement de ces organes et d'autres encore qui n'étaient pas même ébauchés, je serai obligé, pour ne pas tomber dans la confusion, de les suivre désormais un à un ; non-seulement l'exposition y gagnera en clarté, mais j’éviterai encore de tomber dans de nombreuses répétitions, la rapidité avec laquelle tel ou tel organe se forme variant énormémient avec les genres et les espèces.

La formation de la coquille. - J'ai déjà lait voir que l’apparition de la coquille est précédée par la formation d'un renflement de l'ectoderme au pôle aboral, suivie d'une invagination qui se relourne conplétement (Hyaléacées, Styliolacées, Clio) ou en partie (Cymbulie) et forme une saillie plus ou moins conique (Styliolacées) ou arrondie sur laquelle apparaît une cuticule. Cette cuticule n'est autre que le commencement de lat coquille. Elle est raide et cassante dès l'origine et renferme déjà des sels calcaires. Chez les Thécosomes orthoconques, elle est lisse ; clez Cymbulia, elle présente déjà, à sa surface interue, les petites aspérités calcaires qui se trourent sur tonte la corquille de la larre formée.

Dès que le sommet de la coquille a été ainsi formé tout d'une pièce, la saillie coquillière, sur laquelle il s'est moulé, commence à s'effacer. Les cellules qui occupent le centre de la saillie s'aplatissent en s'élargissant et probablement en se multipliant; les cellules des côtés conservent par contre toute leur épaisseur et forment un bourrelet cireulaire, qui entoure une région amincie de l'ectoderme. Cette région amincie est recouverte par la coquille, dont le bord est en regard avec le bourrelet. C'est, en effel, à ce bourrelet qu'est désormais dévolue la fonction de sécréter et d'agrandir la coquille couche par couche, ou plutôt anneau par anneau. Ce mode de formation explique les stries si régulières que l'on rencontre sur les coquilles des Hyaléacées. Ces stries, qui so relrourent aussi che\% d'autres familles où elles sont moins apparentes et moins régulières. sont done des stries d'acerois. 
sement. Chez Caulinia (roir pl. Ill et IV sur toutes les figures), elles apparaissent comme de simples traits, lorsquon les examine à $u n$ grossissement faihle. Sous un fort grossissement (pl. IV, fig. 47), elles apparaissent comme des séries de petits points qui sont l'expression de petites dépressions de la surface externe. Au sommet même de la coquille, se trouve un espace en forme de verre de montre dépourvu de stries; c'est la partie qui a été, pour ainsi dire, coulée d'un seul jet au moment où la saillie coquillière venait de se former.

Chez Clio, la partie arrondie de la coquille est marquée de bandes transversales, larges et grisàtres, qui se trouvent, lorsqu'on les regarde à un grossissement plus fort, n'ètre chacune qu'un ensemble de petites stries verticales (pl. X, fig. 7, qst).

Nous avons déjà vu que la coquille se compose de deux substances, d'une substance organique élastique et de sels calcaires qui l'imprègnent. Traitée par les acides, elle perd sa forme caractéristique et tend à prendre une forme arrondie (voir pl. III, fig. $36, q$ ). Elle ne reste alors attachée à l'animal que par les bords adhérents au bourrelet qui les sécrète.

Ce bourrelet (pl. 1II, fig. 36 , et pl. VII, tig. 9 et 10, qb) remonte petit à petit le long des côtés du corps à mesure que celui-ei s’allonge. En comparant la figure 18 (pl. II) à la figure 99 (pl. III), qui sont dessinées à la chambre claire à un mème grossissement, l'on verra que la larve est presque deux fois aussi longue que l'embryon dont elle sort. A mesure aussi que le bourrelet remonte, la partie postérieure amincie de l'ectoderme gagne en extension; elle se compose toujours d'une couche de petites cellules nucléées et à limites parfaitement nettes (pl. III, fig. 36, ecc).

Le bourrelet du manteau, dont la structure se voit très-bien sur me coupe oplique (pl. III, fig. 36), se compose de deux zones circulaires de cellules cylindriques, séparées par une zone étroite de trèspetiles cellules $\left(q b^{\prime}\right)$. C'est la zone épaisse inférieure $(q b)$, qui sécrète seule la coquille. La zone supérieure $(\mathrm{mm})$ est-elle l'homologue de ce repli du manteau qui, chez les Gastéropodes pulmonés, recourre le bord extérieur de la coquille? N'est-ce pas plutòt le tissu qui formera bientôt la paroi de la cavité branchiale?

Cette dernière supposition est la plus naturelle. Bientòt, en elfet. le bourrelet, et arec lui la coquille, ont atteint la base de la région céphalique, et l’on roit alor's la partie libre du bourrelet s'épaissir, e! commencer à se renfoncer cutre la coquille et le corps, pour former 
la eavité branchiale, qu'il sera plus convenable de nommer ici cavité palléale. L’on sait, en effet, que beancoup de Ptéropodes n'ont pas de branchies.

C'est en ce moment (pl. IfI, tig. 37, pl. V, fig. 9, et pl. X, fig. 7, q) que la coquille a atteint la limite de son développement larvaire. En effet, jusqu'à présent, elle s'est développée d'une manière uniforme et régulière ; il se produit un temps d'arrêt, et puis la coquille recommence à croître, mais d'une manière loute différente et avec un angle d'ouverture plus grand que le précédent. C'est le cas de tous les Thécosomes orthoconques.

La limite entre la coquille larvaire et la coquille de l'adulte est marquée par une ligne très-prononeée (pl. IV, fig. 44 et $46 ; \mathrm{pl}$. V, fig. 4, et pl. VI, fig. 6 et $7, q t)$. Chez Cymbulia quelque chose d'analogue doit avoir lieu, à en juger par la deseription de Krohn. Chez Clis, il se forme en ce point un anneau renflé (pl. X, lig. T, $q t$ ).

Chez Cavolinia, les cellules ectodermiques qui tapissent la coquille prennent, vers la fin de la vie larvaire, une coloration ronge due à un pigment qu'elles renlerment. La même coloration se trouve dans l'épithélium qui tapisse la carité palléale. Le pigment étant répandı seulement dans le protoplasme des cellules et laissant libres leurs bords et leur nucléus, il en résulte une image très-régulière, analogue à celle du tapis noir de l'œil des vertébrés, el qui montre bien clairement la constitution cellulaire de ces couches.

Chez Cymbulia, l'on trouve dans l'ectoderme de l'intéricur de la coquille, du còté ventral et près du bord supérieur, deux ou trois cellules pigmentaires, ranifiées, contractiles, dont le nucléus transparent se détache sur le protoplasme pigmenté en jaune rongeâtre (pl. IX, fig. 19, mch).

La coquille reste-t-elle conme l'a sécrétée le borl du manteau, ou bien se renforee-t-elle intérieurement par des couches d'épaississement? Chez les Hyaléacées, où la coquille reste fort longtemps en rontact arec l'ectoderme, la question est difficile à résoudre d'une manière absolue. Tontefois, j’ai remarqué que la partie embryonnaire de la counille restait toujours à peu près aussi mince qu'au moment où je la vis appraraître. Chez les Stỵliolacées (pl. V1, fig. 6 el 7, et pl. VIl, fig. 9 et 10,4 ), la partie larvaire ne reste en contact avec l'ertoderme que juste le temps néressaire à sa sécrétion. Elle n’adhìre à l'animal que par le rebord palléal el par le point d'attache du musele rolumellairc. Il ne saurait être question ici d'un épaississement. En revanche. 
chez Crescis, l'ectoderme reste très-tarl, sinon loute la vie, en contact avec, la corguille dans toute son étendue el jusqu'au sommet lui-même. Ici l'épaississement progressif de la partie larvaire de la coruille est des plus évidents. J'aurai à revenir, dans la période suivante, sur le sort ultérieur de lit roguille larvaire.

Le développement du vorle. - Nous asons quittó le voile au moment nù il formait un bourrelel circulaire autour de l'extínité antérieure de l'embrỵon. Il élait composé de deux rangées de cellules portant chacune un petit pinceau de cils. Les cils du milieu de chaque cellule étaient les plus longs. Petit à petit, la région céphalique s’élargit en s'aplatissant et le bourrelet s'ćlargit de mème. Les cellules des deux rangées ciliaires s'engrènent l'une dans l'autre et finissent par ne former qu’nue seule rangée. Les cils s'égalisent et ne forment plus aussi qu'un seul rang; ils grossissent et s'allongent, deviennent, en un mot, de véritables cils moteurs ${ }^{1}$.

En même temps, d'autres rangées de cellules, parfaitement régulières et munies aussi de nucléi et de mucléoles, viennent s’ajouter en arrière de la première, le telle facon que chacune de leurs cellules se troure juste en arrière de l'une des cellules de la rangée antérieure (voir pl. IIK, fig. $36, v b$ ). Il résulte de cet arrangement qu'en regardant le bourrelet par dessous (pl. III, fig. 33̆, vb) ou par le dessus (pl. IV, fig. $48, v b$, l'on ne eroit roir qu'une seule rangée de cellules. Line coupe optique, surtout après l'addition d’acide acétique, fait reconnaître la présence de quatre à cinq rangées. Les rangées postéricures portent de petits cils très fins, la rangée antérieure portant seule les cils moteurs, à raison de quatre à cinq cils par cellule (pl. III, fig. 30̃, vc). La taille que peurent atteindre les eils moteurs varie beaucoup, snivant les espèces; ils atteignent leur plus grande longueur $\left(0^{\mathrm{mm}}, 0 \Xi\right)$ chez les Hyaléacées, dont les larves sont lourdes et le roile de dimensions restreintes. Chez les genres dont le roile atteint de grandes dimensions, les eils moteur's sont relativement faibles. Ils ont généralement afleint toute leur croissance d'assez bome heure. lls ne diminuent pas plus tard, mais tombent tels quels, arec les cellules qui les portent.

1 Beaneoup d'auteurs dounent it ces eils le nom de cirres, prétendant ne pouvoir les identifier ì des eils ordinaires. Leurs dimensions sont effectirement exeeptionnelle's, mais rien n'est aussi variable que la dimension de cils vibratiles. Ce qui distingue les cils vibratiles dans tous les cas, c'est leur mouvement partieulier, leur délicatesse à l'égard de certains réactifs et leur mode de développement. Sous tous ces rapports, les cils moleurs sont de véritables eils vibratiles, auxquels je n'hésite pas à donner ce nom. 
Souvent ces cellules renterment, ontre le nucléus, de petits granules, probablement ådipenx, et très-réfringents (pl. 1II, liğ. 35, gr).

Les cils moteurs n'oecupent que le bord supérieur du bourrelet, le reste du bord ćtant couvert de cils tins et nombreux. Chez les IIyaléacées, oì le voile tombe de bonne heure, il ne subil pas d'autres modifications. Mais chez les Styliolacées, les Créséidées, et surtout les Cymbuliacées et les Gymnosomes, où le voile persiste très-longtemps el prend des dimensions ronsidérables, on roit son bord se reuser en goutlière. Le bord supérieur de la gouttière (pl. VI, fig. 7,vee) est formé par un bourrelet épais qui porte les cils moteurs; le hord inférieur est un bourrelet mince (pl. VI, lig. $7, v n$ ) qui porte des cils courts et fins, et enfin tout le fond de la gouttière est tapissé de cils encore plus petits. Le but de cette organisation, comme Claparède l'a montré chez les Rotifères, est de produire des courants qui suivent le fond de la gouttière et amènent les particules nutritives à la bouche. Nous pouvons done donner à ces cils et à ce bourrelet inféricur le nom de cils ef re bomrelet nommiciers, par opposition aux cils et an bourrelet moteurs.

La région céphalique de l'ectoderme, c’est-à-dire la région circonscrite par le hourrelet moteur, porte sourent des cils disposés en lignes on en houppes. Chez C'arolinia, par exemple, l'on voit, un peu du côté dorsal du centre de cette région, une petite proéminence, converte de

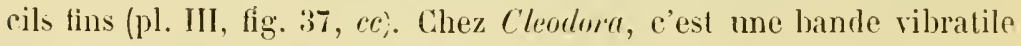
assez large qui part du bourrelet moleur sur la ligne médiane du dos, et se dirige droit rers la bouche, qu'elle n’atteint pas. Un pen en avant du point du bourrelet moteur où se trouve la bouche, elle s'arrête subitement (pl. VII, fig. 9 et 10, cc). Entre l'extrémité de cette bande et la bouche. se trouvent l'abord deux eirres raides, fort gros, qui battent l'eau de loin eu loin d'une manière saccadéc $(c \%)$. Ils sont portés chacun sur une cellule ectorlermigue proéninente. Entre ces cirres et la bouche, se trourent encore leux cellules proéminentes, couvertes rhacune d'une houppe de cils tins.

Il y a une grande variété dans la forme et la distribution de ces cils réphaliques, et la description que j’ai domnée de C'leodora montrera que cette distribution n'est pas toujours très-simple. Leur présence chez les Lamellibranches et chez Chiton a élé signalée depuis longtemps, el il est intéressant de les retrouver chez d'autres Cóphalophores.

Le roile s'élargit et derient mobile. par suite de la formation de 
fibres musculaires, que je décrirai plus loin. L'élargissement ne concerne que les parties lalérales, qui se transfornent ainsi en le réri-

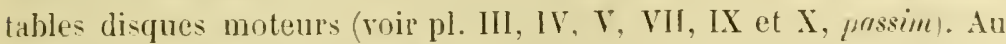
milieu du côté rentral et du côté dorsal, le bourrelet tend plutòt à leculer, formant ainsi d'ux profondes échancrures (pl.IV, fig. 48). Léchancrure la plus profonde se trouve du còté rentral, et c'est au fond de cette échancrure que l'on rencontre l'ouverture de la bouche. En cet endroit, le bourrelet reste toujours très-mince et les cils y sont trèscourts, de mème que dans l'échancrure dorsale. Nous aruns ru que le bord de la bouche et le bourrelet roilier ne sont pas nettement - séparés chez l'embryon. 11 en est souvent de mème plus tard; la bouche peut paraître placée entre les deux lobes du voile, ou bien le bourrelet roilier peut finir par former une légère saillie au-dessus de l'ouverture buccale. On voit par là combien les différences dans la position relative de la bouche et du bourrelet locomoteur ont peu d'importance.

Je note en passant, puisfue quelques auteurs ont ma! compris ce point, que le bourrelet roilier n'est que le bord épaissi d'un repli de l'ectoderme. Ce bourrelet est done relié au corps par deux membranes, dont l'une se continue aree l'ectorlerme du cou et de la base du pied, l’autre avec l'ectoderme de la région réphalique. L'espace compris entre ces deux membranes est rempli par le liquide nourricier, et parcouru par les fihres musculaires ramifiées.

L'origine première des fibres musculaires du voile remonte assez. haut. Pour la trourer, reprenons la larre atu moment oì son bourrelet roilier commence ì se relever sur les côtés de la région céphalique (pl. III, fig. 29). L'on voit, en ce moment, quelques cellules se détacher. je erois, de l'ectoderme et faire saillie dans la cavité du corp̧s, étendues entre le roile et la masse nutritive. Ces cellules montrent bientôt de fines ramifications à leurs extrémités, surtoul à l'extrémité externe. A mesure que le voile grandit, ces cellules s'allongent (pl. IiI, fig. 3ä, ¡. . Chacune n’a qu'un seul nucléus. Leurs ramifications se multiplient et vieunent s'insérer à la face intęrne du bourrelet moteur. Leur contractilité ne tarde pas à se montrer de la façon la plus évidente, et r'est aver le rléveloppement de ces tibres que le voile acruiert sa grande mobilité. Il y a, en général, trois à qualre fibres ramiliées de chaque côté. qui se fusionnent, fle façon à n’en former que deux (roir pl. IT. fig. 48, ґ.): leur disposition rarif du reste d'un individu à l'autre. et même entre les deux côtés d'un même individu. Au moment 
où elles ont arupuis déjà un dertain développenent, l’on discerne la membrane, comparable à la membrane de toute autre cellule, qui les enveloppe, at un contenu protoplasmique, qui ne présente aucune trace do stries. l,e nucléus a disparu. Ces muscles sont destinés à disparaitre au moment oì le roile tombe. Cette forme de cellules musculaires n'a rien qui nous élonne, ear nous savons qu'elle r'sl extrèmement répandue chez les animaux pélagiques : Ciénophores, Mollusques, ele.

Deux itutres cellules musculaires lannifiées se trourent au bord rentral du voile, où clless šinsèrent à l'ectoderme dans le voisinage de la bouche d'une part et au bourrelet moteur' d'autre part (pl. IV, fig. 48, ; $n$ ). Ces libres ne diffërent en rien des précérlentes, mais elles ne se forment que plus tard, maissant de quelques cellules qui paraissent liées à l’invagination nerveuse, comme nous le verrons plus loin.

C"est chez les Styliolacées, les Clioüdées et les Cymbuliacées que le voile atlemt les plus grandes dimensions. La figure 7 (pl. VI), où est représentée une larve de Styliola au moment où cet organe at atleint son maximum, domnera une ilée de son importance; le roile est représenté à moitié replić. A l'élat d'extension, il est d'un ticrs au moins plus grand qu'il n'est représenté sur la figure. Chez Creseis (roir p. j6) chaque moilić so sépare en deux lobes par une échancrure du bord cxterne.

Le déceloppermert du pied. - Lat posilion du pied est déjà clairement indiquéesur des embryons dont le fenillet externe vient de so fermer. Il se troure à la lace reutrale, entre l'invagination próconchylienne et le point de rencontre des trois cellules nutritives oì se formera la bouche. Nous arons déjà vu comnent il commenee à se différeneier. Il a la forme d'une bosse; ses parois sont composées d'un f́pithile simple. Un espace, répondant à une partie de la cavité du corps, se forme entre ses parois et la masse nutritive (pl. V1, fig. 1, lic). Caest lans cette cavité que l'on voit apparaître des cellules isolées, quii proviennent, sans doule, d'un dédoublement de quelques cellules ectodermiques (pl. V1, lig. 1, \%. c). Destinćes à former par la suite des fibres musenlaires, ces cellules restent, pour le moment, inmobiles el saus subir de changements. A la partic postérieure du pied se trouvent les denx cellules qui indiquent la position de lanus (pl. II, fig. 2l, et pl. VI, fig. 1, ac).

I mesure que le rorps s'allonge en arrière el que le borld de la roquille remonte, toute la partie de l'ectoderme qui ne prend pas part 
à la formation de la coquille, et qui forme plus de la moitié antérieure de l'embryon, se retrousse en quelque sorte. Les masses nutritives se retirent vers la partie entourée de la coquille, et la moitić céphalique, comprenant le pied, l'anus, le voile et la muque, perd en hauteur et sélale en largenr (comparer la figure 19, pl. II, aree les figures 29 el 37 , pl. IIl). Le pied, de simple bosse, devient un lobe arrondi, déprimé, quoique encore assez épais; les deux cellules anales se trouvent inmédiatement au-dessous rle sa face inférieure (pl. III, fig. 29, ac el l\%). Vu d'en haut, le pied a déjà une forme de languette pointue au bout et élargie sur les côtés (pl. III, fig. 41, $\mu$ ). Il se couvre de eils vibratiles fins, surtout à la face supérieure, où ces cils battent l'eau arec vivacité. Les cils qui garnissent le bord sont peu mobiles.

A u moment où la coquille a atteint l'endroit le plus large du corps, le pied commence déjà, ehez les Hyaléacées, à se diviser, par denx échancrures, en trois lobes (pl. HI, fig. 42). Ces échanerures ront en s'approfondissant, et les lobes se trourent presque entièrement séparés au moment où le bord de la coquille a atteint la base du pied et où la cavité branchiale est en voie de formation (pl. III, fig. 40 et $37, \mu$ ' et $m$ ). Le lobe médian, on l'a déjì deviné, deviendra le pied proprement dit, tandis que les lobes latéraux formeront les nageoires.

Au point où nous en sommes, les lobes latéraux (oommencent à se relever el à pousser en hauteur, tandis que le lobe médian reste aplati (pl. IIl, fig. 37, $m$ et $p$ ). Dans l’intérieur, les cellules mésodermiques commencent à prendre une disposition régulière le long du bord externe du lobe médian. Des fibres musculaires transversales se sont formées à sa base (pl. III, fig. 42, lip), constituant une sorte de sinus contractile, qui se resserre et s'élargit à intervalles irréguliers, mais assez rapprochés. Ces contractions alternent avec celles de la région dorsale, et prourent l'existẹnee, chez quelques Ptéropodes, de cette circulation embryonnaire déjà décrite chez tant d’autres Céphalophores (roir p. 76 et suiv.).

Les lobes latéraux se mettent maintenant à pousser lapidement en hauteur (roir pl. III, fig. 38, et pl. V, fig. 2, m m). Les cellules mésodormiques s’accumulent dans leur partie supérieure, et se présentent, rues de protil, comme des corpuscules fusilormes attachés par leurs deux pointes aux deux parois opposées (ul. V, fig. 2, p.ps). Le bord des trois lubes est muni d'une rangée de cirres lailes, qui alteignent leur plus grande longueur à l'extrémité du lobe médian (pl. V, fig. 2. crp)). 
Les bases des nageoires sont élargies, s'étendant reps le dos sous la naissance du voile et prenant la bouche entre leurs bords dorsaux.

Nous aurons à suivre, pendant la prochaine période, le développe. ment ultérieur de ees organes. Mais, avant de quitter ee sujet, passons d'abord en revue les modifications que ce mode de déreloppement subit chez les principaux types du groupe.

Chez les Créséidées, le développement des nageoires a lieu encore assez rapidement (pl. Vl, fig. $.5, m)$ et ne diffère guère de celui des Hyalćacées.

Chez Ies Styliolacées, la formation des nageoires doit être trèstardive, puisque, sur la lapre représentéc sur la planche VI, fig. 7, le pied $(p)$ n'est encore que simplement triangulaire, sans échancrures latérales.

Chez les Cymbuliacées, le pied, garni d'une forte toison de cils à sa face supérieure, est muni d'un opereule à sa façe inférieure. Il est eneore simplement arrondi sur la larre de la figure 19, pl. IX, et n'a pas encore de traces dle ces élargissements considérables auxquels il donnera naissance plus tard, d'après les observations de Krohn (p. 63).

Che\% les Cliöldées, enfin, le pied se montre de bonne heure, mais il reste d'abord extrèmenent petit. 11 occupe la même position que rhez les Thécosomes, et forme, immédiatement au-dessous de la bourhe, me petite languette en forme de luette et repliée en gouttière (pl. X, fig. $\bar{\tau}, p)$. L’extrémité le ce pied s'allonge et s'étire ensuite en une lanière couverte de cils vibratiles, et que l’on décourre aisément entre les deux grands disques moteurs. Le développement nltérieur du petit pied en fer à cheval et des nageoires, ainsi que la chute de la lanière pédieuse viluratile. tombent en dehors de la première période larraire des Gymnosomes. Je n'ai pas en l'oceasion d'observer ces changements; mais, d'après les ubservations de Krohn, ils pourraient se ramener au mème type de développement que ehez les autres Ptérnpodes. I'insiste seulement sur ce fait, que le pied se forme aussitôl guce rhez les autres Ptéropodes, c'est-ì-dire an début de la périotle larvaire, et que la lanièze vibratile n’est qu'me excroissance de l'extrómité du pierl.

La formutiom de la carité pulléale. - Nous arons déjà vu que, an moment où la coquille larvaire a atteint la base du pied et du voile, il se produit un petil temps d'arrèt dans son développement, temps pendant lequel la cavité hranchiale enmmence à se former. Chez les Thérosomes orthoconrues. ce point d'arrît est nettement marqué sur la 
coquille, qui grandit ensuite sur un modèle dillérent. Clez clio, la coquille est déjà surmontée d'un anneau avant que la cavité hranchiale soit indiquée (pl. X, fig. $7, q t$ ).

L'ouverture de la coquille est, en ce moment, plus large que la portion du corps qu'elle entoure, et que, pour faciliter la description, j'appellerai tout simplement le cou. Le bord du manteau entoure le cou comme un col trop large et passe, du reste, saus interruption it l'ectoderme de la région céphalique en formant un angle rentrant dans lequel l'ectoderme est épaissi. Cet épaississement n'est antre que la moitié supérieure du bourrelel coquillier (pl. 111 , fig. $36, \mathrm{~mm}$ ). C'est cette partie épaissie qui commence maintenant à se renfoncer vers le bas, el cela d'abord du côté droit chez les Orthoconques, entre la base du pied el la base du disque moteur de droite (pl. VI, lig, $(i, m c)$. Bientôt la partie renfoncée s'allonge ver's le dos et aussi un peu du côté rentral, en sorte que l'entrée de la carité prentl la forme d'un eroissant. La cavité elle-mème est comprimée et s'étend rapidement vers le bas (pl. III, fig. 37,mc). La paroi périphérique est fort épaisse et séparée de l'ectoderme, qui tapisse la coquille, par un espace rempli de licpuide nourricier et faisant partie de la cavité du corps (pl. III, fig. 37, et pl. V, fig. 2, $k c$ c). Cette paroi épaisse montre chez certains genres un système de atries transrersales, qui est l'expression de l'arrangement régulier des cellules épithéliales en lignes transversales (pl. V, fig. 2 et s., $m b$ ). Elle deviendra le bouclier vibratile, bien connu, des Ptéropodes. Le fond du repli a aussi un épaississement local, qui provient de l'endroit où la carité palléale a eommencé ì se former, et qui deriendra la glande rénale.

En s'étendant et s'approfondissant, la cavité palléale finit par entourer de trois còtés le corps ou sac viscéral, qui ne reste en contact arec la coquille que par mu eòté, le côté gauche et dorsal, chez les Orthoconques. Chez les Thécosomes ampyloconques, la position de cette cavité varie avec l'àge de la larre, arec le degré et le genre de courbure de la coquille el la torsion du corps qui en résulte. Elle se tronve, à son origine, du côté dor'sal, en arrière de l'anus.

C'est du côté dol'sal, rers le point d'insertion du muscle columellaire, que linvagination palléale alteint sa plus grande profondeur (pl. 111, fig. 38 et $39, m c$ ). Mais, mème sur le reste de la périphérie du con, l'on voit régner un sillon'qui le sépare du bord palléal, et qui n'est que la continuation de la cariti du branchial.

La parui interne de eette carité, celle qui la sépare du corps, est plus 
mince que l'antre et montre de bonne heure une différenciation remaryuable. En effet, il s'y forme une série de fibres musculaires horizontales, applicpuées par toute leur longueur à la surface interne de la membrane. qu'elles font onduler. La formation te ces fibres a lieu, sans doute, anx dépens des cellules épithéliales de la paroi par dédoublement; car la membrane derient extrêmement mince dès que les fibres musculaires se montrent à son intérieur ${ }^{1}$ (voir pl. III, fig. 37 et 39, et pl. V, tig. 2, lin). Cette membrane contractile courre toute la partie droite du sae viscéral et s'élend jusqu’an côté dorsal. Ses contractions sont d'abord plus complètes et alternent arec celles du simus du pied. Plus tard le sinus pédieux disparaît, mais la membrane contractile dorsale continue à exécuter des mouvements ondulants de bas en haut, mouvements qui se ralentissent et se perdent à mesure que le cromr se déreloppe et entre cn fonetions. Pendant un certain temps, l'on roit ces deux organes simultanément en activité. Ce temps est assez prolongé chez les Créséidées (pl. Vl, figg. .̈. linn), où la résicule contractile dorsale est très-active, mais restreinte à la partie sitnée cutre l'estomar el le con, ef où le crour n’apparaîl que trèslarkl.

Lorsque l'animal se retire dans sa coquille, le flnide nourricier yui blait contenu dans le voile et le pied refine vers cette région: la membrane en question se tend et se remplit anx dépens de la cavité palléale, qui se vide, et dont les deux parois viennent s'appliquer l'une contre l'antre.

Nous retrouvons done ehez les pléroporles cette forme de circulalion larvaire qui a été observée dhez les Prosobranches et une foule d'antres Céphalophores. D'ahord ce sont denx sinus contractiles, situés l'un dans le pied et l'autre dans le dos, qui se renvoient le liquide; puis, le sinus pédicus ressant de fonctionner, le sinus dorsal chasse le lipuide nourricier par des contrartions ondulantes, et ce liquide est encore mis en mourement par la rétraction et l'expansion alternatives du voile. Que l'on observe des larves nageant librement dans un grand bocal, et l'on verra qu'elles étendent leur voile et montent dans l'eau. puis le relirent et se laissent tomber, et ces mouvenents alternatifs se font arec une grande régularité. Il ne peut être douteux que ces flats alternatifs d'expansion el de rétraction ne jouent un rôle im-

1 J'emploie les mots interne ext exlerne on périphérique, en les rapportant ì lanimal tout entier, et non à la cavilé palléale. 
portant en mettant le fluide nourrieier eñ mouvement et en chassant de temps en temps dans tout le corps le liquide qui s'est oxygéné dans le voile. Les cils de la eavité palléale n’apparaissent que fort tard, et, en attendant, ee sont, sans doute, les conrants produits par les cils moteurs et les rétractions de la tète, qui renouvellent l'ean dans cette cavité.

A mesure que la cavité palléale s'étend, elle gagne anssi en épaisseur, et sa paroi externe vient presque s'appliquer eontre l'eetoderme qui lapisse la coquille. Au bord supérieur du manteau, cependant, ees denx parois laissent entre elles un espace, un sinus circulaire (pl. III, lig. 38 el 39, el pl. V, fig. 2, cm). Déjà, an moment où se formait la ravité palléale, l'on voyait, entre sa paroi et l'eetoderme, des cellules musculaires, ramitiées aux deux bonts et tendues d'une paroi à l'autre (pl. 1II, fig. 3T, $:$ m). Ces fibres ont au milieu un renflement qui renferme un nucléus. Elles servent à chasser, par leur contraction, le liquide nourrieier que contient le sinus. Elles sont encore bien déreloppées à la fin de la vie larvaire (pl. V, lig. 2, p.m), mais ront en diminuant par la suite.

Au bord inférieur du sinus, du côté droit et dorsal, se trouve un bourrelet de cellules qui fait saillie dans la cavité palléale; c'est un simple épaississement de l'ectoderme, qui se couvre de grands rils vibratiles (pl. IIf, lig. $39, m x)$. Nous aurons à reparler de cet organe vibratile, après la métamorphose, à propos des organes des sens.

Le développentent du tube digestif. - Nous arons déjà vu comment la cavité stomacale se forme au point de rencontre des trois sphérules nutritives primitives. Cette cavité est ovale et allongée dans le seus de l'axe longitudinal (pl. II, fig. 19, et pl. VI, fig. 1, s). Ses parois sont formées de cellules aussi hautes que larges, composées d'une membrane minee, d'un protoplasme et d'un nueléus. Cependant une observation altentive montre que cel épithèle n’est pas entièrement refermó sur lui-mème. Il laisse, au contraire, chez les Hyaléacées une ourerture assez large à la partie dorsale et inférieure; pent-être y a-t-il deux ouvertures au lien d'une. Je ne peux pas préciser ce point. Chez r'lir, je trouve deux ouvertures bien évidentes, communiquant au còté gauche et au côlé ventral avec deux earités distinctes (pl. X, lig. 6 et $\bar{i}$, s'). Qu’il y ait une ouverture menant dins une cavité double, on qu'il y cn ait deux menant chacune dans une carité distincte, la structure de la carité, qu'elles mettent en communication arec l'estomac, restetoujours la même. C’ent nne eaviló ('reuséc dans la masse 
des cellules nutritives, qui premnent autour d'elle le mème arrangement radiaire qu'elles avaient au moment de la formation de la carité stomacale primitive. Elles sont done cunéiformes et présentent leur sulstance formative à leur partie interne (pl. $\mathrm{X}$, lig. 7, ) ). Là où il y a deux de ces masses, elles ont à peu près la même apparence et la même forme extérieure que les cellules nutritives embryonnaires dont elles descendent. Mais un examen plus attentif montre que leur structure intime a complétement changé et que chaque masse se compose mitintenant de plusieurs unités histologiques, de plusienrs cellules, au lieu de répondre à une seule cellule (pl. X, lig. 6 et 7, $\left.5 \sigma^{\prime}\right)$. Ces cæecums, dont la paroi est formée de cellules nutritives, el dont la cavité débouche dans l'estomac, ne sont autres que les sacs nutritifs.

Ainsi done la paroi du tube diggestil se compose de cellules résultant d'une scission des cellules nutritives, après quoi ces dernières s'arrangent autour d'une carité double ou de deux carités simples qui restent en communication ouverte avee le tube intestinal.

Ces différenciations de la partie centrale du tube digestif sont à peine terminées, que déjà nous royons l'rrsophage et lintestin se former et s'aceuser. Commençons par l'u'sophage.

Nous arons vu la bouche se former par une invagination de l'ectoderme située, selon toute probabilité, au pòle nutritif. Nous avons assisté à l'allongenrent de celle earité et à sa transformation en un lube fermé en recum et dirigé en bas dans le sens de l'axe formatif (pl. VI, fig. 1, et pl. X, fig. 6, $\infty$ ). Nous arons ru enfin qu'arant d'atteindre la eavité stomacale primitive, ce cæecum s'élargit à son extrémité inférieure (pl. III, f.g. 36, $\omega^{\circ}$ ). Cet élargissement a lieu surtout dans le diamètre rentro-dorsal. Puis la partie dorsale de l'élargissement continue à pousser; elle rencontre la carité stomacale, se finsionme avec elle; le point de fusion se perce et devient le cardia. Lia partie rentrale de l'élargissement de l'esophage reste en arrière, se développera plus tard et deviendra le sac qui contient la radula. L'resophage lui-mème n'est, pendant toute la vie larvaire el encore au delà, qu'un simple tube composé d'un épithèle vibratile simple.

La cavité digestive embryonnaire est dirigée parallèlement à l'axe longitudinal et se troure beancoup plus près de la face ventrale que de la face dorsale. Son extrémité inférieure derra, par conséquent, ètre tournée vers lectoderme. Elle est, en effet, très-rapprochée du point de liı surlace où se trouvent les denx cellules anales. Lespace, entre cen deux parties, est laissé libre par les grusses cellules nutri- 
tives et n'est occupé que par des cellules allongées, clair-semées et à contours peu nets (pl. VI, fig. 1, en). Ces cellules proviennent-elles de l'entoderme, c'est-à-dire des cellules nutritives? C'est fort probable, quoique je n'aie pas observé directement leur mode de formation. Elles auraient difficilement pu se détacher de l'ectoderme sans que je m'en aperçusse: Servent-elles à la formation de l'intestin? Il est possible qu'elles y contribuent, mais la majeure partie, sinon la totalité de l'intestin, se forme par prolongement de la cavité digestive embryonnaire et ne saurait done se former aux dépens de ces cellules, qui ne peuvent trouver leur emploi que dans la formation de l'anus.

L'extrémité inférieure et ventrale s'étire en forme de tube à peu près comme le col d'une cornue, et finit par venir se souder avec l'ectoderme. Ceci a lieu à un moment où le cardia est déjà percé, où la coquille remonte sur les côtés du corps et où la base du pied et la région anale (pl. IIl, fig. $29, a c$ ) remontent rapidement dans la direction de la bouche. Il en résulte qu'avant même le percement de l'anus, l'intestin, qui est encore très-massif, semblable à un gros cordon cellulaire et muni d'un lumen presque nul, se trouve étiré et recourbé vers le haut (pl. IlI, fig. 37, i). Alors seulement l'anus se perce, le canal intestinal prend une certaine largeur et se tapisse de cils. L'œsophage et l'estomac se sont couverts de cils peu après la formation du cardia; la bouche est ciliée dès son origine. Le tube intestinal est done muni de cils dans toute sa longueur.

C'est aussi en ce moment que commence, chez les Hyaléacées, la différenciation de la paroi de l'estomac en une couche interne épithéliale et une couché externe, qui se transforme en un système de fibres musculaires (pl. III, fig. 39, p.s) disposées en cercles autour de cet organe. Ces fibres iront en se multipliant et formeront une couche puissante. En même temps, les cils qui tapissaient l'intérieur de l'estomac, disparaissent. Peu après la formation de la couche musculaire, la couche muqueuse ou épithéliale se met à former des replis, qui font saillie dans la carité de l'organe, et sur lesquels se formeront les dents stomacales. L'estomac prend de plus en plus la forme d'une poire dont l'œsophage représenterait la queue, tandis qque l'intestin naît au gros bout et un peu sur le côté rentral.

Chez les Styliolacées, l'estomac, qui deviendra de plus en plus pareil en tous points à celui des Hyaléacées, commence par affecter une forme larvaire qui se retrouye chez les Cymbuliacées el les Clioïdées. Il s'arrondit, prend une position transversale (pl. VI, fig. 7 ; pl. IX, ARCH. DE 2 กOI. EXP. ET GÉN. - T. IV. 1875. 
fig. 19, et pl. X, fig. T,s). Dans son intérieur, l'on voit une plaque transparente, rigide, appliquée contre la paroi ventrale de l'estomac dans la moitié voisine du pylore, et garnie de petites dents perpendiculaires à sa surface et saillantes par conséquent dans la cavilé de l'organe (pl. VI, fig. 7, dl). Cette plaque dentée a déjà été observée par Krohn (voir p. 47). Elle tombe ou se résorbe ; bref, elle disparaît pour faire place à des dents coniques, pareilles à celles de Hyalea. Cette plaque dentée ne se montre donc que chez les espèces qui virent trèslongtemps à l'état de larves.

L'intestin est, comme nous l'avons vu, d'abord extrêmement court et rectiligne, puis il se recourbe et s'étend vers le haut.

Chez les Thécosomes orthoconques, l'anus se trouve originairement sur la ligne médiane au-dessous du pied; chez les Styliolacées, où la cavité palléale ne se forme que tard, il débouche ici directement à l'extérieur (pl. VI, fig. 6, a). Chez les Hyaléacées, l'anus est entraîné dans la direction aborale, avant même qu'il soit percé, par le repli de la cavité palléale. Cette cavité est d'abord peu profonde sur la ligne médiane ventrale, où se trouve l'anus; petit à petit elle s'étend aussi dans cette direction, et l'anus reste alors sur la paroi interne de la cavité et se déplace vers la gauche (comparer pl. III, fig. 37 et 38, et pl. $V$, fig. 2 et $3, a)$.

Chez les Créséidées, le même déplacement a lieu, mais il est plus tardif; chez les Styliolacées, il est à la fois tardif el peu considérable, l'anus ne déviant que peu vers la gauche.

Chez les Campyloconques et les Gymnosomes, au contraire, l'anus apparaît à droite et y reste.

La position de l'intestin dépend naturellement de celle du pylore et de l'anus, c'est-à-dire qu'il est situé dans le plan sagittal chez les Orthoconques, et du côté droit chez les Campyloconques et les Gymnosomes. Chez les premiers, il forme une anse simple dont la convexité est tournée en bas (pl. III, fig. 38, i); chezles derniers, il forme une anse dont la convexité est tournée en bas et vers le côté dorsal et droit, puis décrit une seconde courbure, moins accentuée, en sens inverse, avant d'atteindre l'anus (pl. IX, fig. 19, et pl. X, fig. 7, i).

Nous avons laissé le sac nutritif au moment où il formait deux lobes creux et où ses cavités communiquaient ouvertement avec l'estomac primitif.

Chez les Hyaléacées et les Créséidées, l'un des deux lobes nutritifs est, dès l'abord, beaucoup plus grand que l'autre (pl. IIl, fig. 39, et 
pl. V, fig. 2, $\left.\sigma \sigma^{\prime}\right)$. Le petit lobe disparaît rapidement, ou se fusionne avec le grand, qui forme seul un sac nutritif unique dont la cavité débouche dans la partie postérieure de l'estomac. A mesure qque la larve grandit, la masse nutritive diminue non-seulement d'une manière relative, mais même d'une manière absolue (comparer pl. III, fig. 37,38 et 39, ヶ).

Chez les Styliolacées, I'on trouve deux sacs presque égaux entre eux (pl. VI, fig. 6, $\left.\sigma^{\prime}\right)$, dont un seul, celui de droite, s'allonge plus tard (pl. VI, fig. 7,, ).

Chez les Campyloconques et les Gymnosomes, il y a deux sacs nutritifs de grosseur à peu près égale, débouchant vers le milieu de la longueur de l'estomac, l'un au côté ventral, l'autre au côté gauchẹ (pl. IX, fig. 16, 17, 18 et 19, et pl. X, fig. 6 et $7, \sigma \sigma^{\prime}$ ).

Tandis que le sac diminue, l'estomac se différencie plus nettement de l'intestin, et l'on roit alors que le sac nutritif débouche, non plus dans l'estomac, mais dans le pylore même.

Les parois du sac subissent des modifications notables. D’abord, les cellules qui les composent se multiplient rapidement par division, et deriennent de plus en plus petites. Puis, dans la substance nutritive réduite, l'on voit se former des gouttes de graisse, qui proviennent sans doute d'une modification chimique de cette substance.

Le sac se contracte régulièrement par un mouvement péristaltique qui part d'en bas et chasse ainsi son contenu dans l'estomac. Puis celui-ci, se resserrant à son tour, renvoie le liquide dans le sac et dans l'intestin. Ce liquide est chargé de globules de graisse et de substances granuleuses, que l'on voit se détacher des parois du sac nutritif. Ainsi s'explique la réduction rapide de la provision de substance nutritive; elle arrive dans le tube digestif, où elle est simplement digérée!

Cette matière nutritive doit éridemment appartenir aux cellules qui tapissent et continueront à tapisser la paroi du sac. Par quel procédé histologique arrive-t-elle dans la cavité de l'organe? Est-ce par une rupture des cellules ou par une simple sécrétion? Les images que j’ai vues me font incliner vers la première hypothèse. Je renroie à la prochaine période la description du sort ultérieur du sac nutritif et de la formation du foie.

Pendant la formation du tube digestif, l'ectoderme, d'abord incolore chez Cavolinia, a commencé à se pigmenter en rouge, coloration qui va en augmentant jusqu'après la fin de la vie larvaire.

Le développement des otocystes. - J'ai déjà dit (p. 129) que les oto- 
eystes se montrent au premier abord comme de simples vésicules a parois très-épaisses, appartenant à la eouche dermique, qui règne sur les côtés du pied. J'ai montré aussi que cette couche provient d'un dédoublement local de la couche ectodermique. Bientôt après que le dédoublement a eu lieu, les cellules ectodermiques se multiplient et deviennent plus petites par division, tandis que les cellules mésodermiques conservent les dimensions des cellules embryonnaires, qui constituaient l'épaississement latéral du pied. J'ai représenté (fig. 28, pl. II) ces deux conches superposées; les contours des cellules ecto. dermiques et leur's nucléi sont indiqués par les traits foncés; les cellules mésodermiques, par les lignes claires. L'on voit l'otocyste se différencier des cellules mésodermiques avoisinantes, auxquelles il ressemble beaucoup à son origine; il est situé exactement au même niveau et ne diffère des autres cellules que par des dimensions un peu supérieures et par la présence d'une grande vacuole.

Il serait possible d'après cela que l'otocyste prît son origine par la modification d'une seule cellule mésodermique, qui produirait une vésicule dans son intérieur (Secretbläschen); mais il ne serait pas impossible non plus que la paroi de la vésicule prît naissance par la fusion de deux ou de plusieurs cellules.

La forme de l'otocyste, en ce moment (pl. II, fig. 26), n'est pas simplement ronde chez Cavolinia; son bord présente trois échanerures, qui divisent la paroi en autant de lobes. Mais, si l'on ajoute une goutte d'acide acétique, il prend une forme arrondie (pl. II, fig. 27), et je n'ai réussi à discerner dans sa paroi qu'un seul nucléus ( $y)$, renfermant un nucléole brillant $(o l)$ qui a la même apparence que le premier rudiment d'otolithe. Chez Cymbulia (pl. IX, fig. 20) l'otocyste a aussi des échancrures à son bord et possède une paroi, dont l'épaisseur dépasse dans l'origine le diamètre de l'espace vide. L'otocyste de Hyalcea striata est arrondi, et j'ai réussi à distinguer, dans la moitié orale de sa paroi, plusieurs nucléi (pl. VI, fig. 1, $\omega)$. Celui des Styliolacées est arrondi aussi (pl. VlI, fig. 10, $\omega$ ). Chez Clio, cette vésicule est elairement composée de plusieurs cellules et prend même parfois un aspect mùriforme avant l'apparition de l'otolithe (pl. $X$, fig. $6, \omega)$; dans ces eals la earité centrale de l'amas paraissait, à l'origine, communiquer aree l'extérieur par un fin eanal. Chez tous, la moitié supérieure ou céphalique de la paroi est plus épaisse que la moitié postérieure.

Un fait m'a frappé, chaque fois que j'ai suivi la formation de l'otolithe dès sil première origine: c'est que l'otolithe apparaît d'abord dans 
l'épaisseur de la paroi de l'otocyste, dans sa partie épaissie, c'est-à-dire dans sa moitié orale, et qu'il vient ensuite lomber dans la cavité.

Quelle est, histologiquement parlant, l'origine de ce premier rudiment d'otolithe ? Les préparations à l'acide acétique (pl. II, fig. 27, $\omega$ ) sembleraient indiquer que sa formation a lieu dans l'intérieur d'un nucléus de la paroi; que ce ne serait, en d'autres termes, qu'un nucléole d'une composition chimique particulière. Mais il ne faut pas perdre de vue la possibilité que cet espace ovale (v) ne soit pas un véritable uucléus. Gela pourrait être une vésicule intracellulaire (Secretbläschen), et alors l'otolithe se formerait de la même manière que les concrétions rénales dont Gegenbaur (voir p. 77) a si lien décrit le développement. J'ai fait mon possible pour résoudre cette question. mais je n'ai pu y parvenir. Sur des sujets vivants, la position de l'otocyste sous la couche ectodermique et la rotation rapide de la larve, dans des préparations coagulées, la teinte foncée que prend l'ectoderme, rendent l'étude également difficile, que l'on emploie l'une ou l'autre de ces méthodes d'observation.

Quoi qu'il en soit, une chose reste certaine, ì savoir : que l'otolithe prend son origine dans l'épaisseur de la paroi de l'otocyste, du côté céphalique. Il sort ensuite petit à petit de la paroi, à laquelle il reste attaché un certain temps encore; puis il tombe dans la vésieule. Il n'est cependant pas encore entièrement libre dans le liquide qui remplit la cavité vésiculaire; il est immobile, ne changeant pas de position, malgré les mouvements de l'animal, et quoique l'on soumette l'otocyste à une légère compression. Il s'écarte lentement de la paroi dans laquelle il a pris naissance, el ne se dégage entièrement qu'au moment où il a atteint le centre de la vésicule et où les cils vibratiles commencent à se mettre en mouvement. Je suppose, sans l'avoir vu, qu'il doit rester enveloppé, jusqu'à ce moment, d'une couche transparente de substance protoplasmique qui serait en continuité avec celle de la paroi.

Le premier rudiment d'otocyste, jusqu'à une grosseur d'environ 2 millièmes de millimètre, n'est pas modifié par les acides, ce qui prouve qu’il ne rcuferme pas encore une quantité appréciable de calcaire. Plus tard, l'action des acides provoque une effervescence marquée, mais nême alors et après l'extraction du calcaire il reste un corpuscule de même forme el de même grosseur que l'otolithe. Ce dernier se compose donc d'une substance cornée imprégnée de calcaire. Un otolithe de 1 centième de millimètre de diamètre environ 
a des stries concentriques, répondant à des couches d'épaississement, et se fend par la compression en trois segments égaux qui répondent, sans doute, à la texture cristalline du calcaire dont il est imprégné.

Depuis sa première apparition conme simple petit point réfringent jusqu'au moment où, devenu entièrement libre dans le liquide de l'otocyste, il a atteint à peu près ses dimensions définitives, l'otolithe ne cesse de croître d'une manière uniforme. Le moment où il sort de la paroi, pour faire saillie dans la cavité, varie beancoup suivant les genres. Chez les Hyaléacées et les Styliolacées, cette sortie a lieu de très-bonne heure (pl. VII, fig. 10, (w), à un moment où l'otolithe ne mesure guère que 1 ă dix-millièmes do millimètre; chez Clin (pl. X, fig. $7, \omega l)$, elle a lieu déjà un peu plus tard. Chez Cymbulia, l'otolithe reste très-longtemps dans la paroi, où il atteint la dimension considérable de $7 \check{5}$ dix-millièmes de millimètre (pl. IX, fig. $20, \omega l)$. Sa sortie a lieu plus brusquement dans ce cas et l'apparition des cils vibratiles la suit de près.

Il résulte de ce que j'ai dit que la formation de l'otolithe larvaire a lieu dans les cellules de la paroi et non par un simple précipité du liquide de la vésicule. Sclon mon opinion, sa croissance se ferait presque entièrement pendant une période où il serait entouré d'une couche protoplasmique qui appartient à la paroi cellulaire, quoiqu'elle fasse hernie dans la cavité de l'otocyste. La précipitation de sels calcaires du liquide de la vésicule ne jouerait done aucun rôle important même dans la croissance ultérieure de l'otolithe.

II n'y a qu'un otolithe par vésicule auditive pendant toute la durée de la vie larvaire.

L'apparition des otolithes, le moment où ils font saillie dans la vésicule sont des phénomènes dont le moment exact peut être observé avec précision; aussi sont-ils particulièrement précieux pour estimer le degré d'asymétrie qui se manifeste dans la croissance des deux côtés de la larve. L'otocyste de droite apparaît une demi-heure ou une heure et mème davantage arant celui de gauche. La même différence s'observe dans le moment d'apparition de l'otolithe. Un peu plus tard, l'on voit à droite un otolithe plus gros qu'à gauche (pl. X, fig. 7, $\omega l$ ), et souvent l'otolithe sort déjà de la paroi de la vésicule du coté droit, tandis qu'il y est encore enfoncé dı côté gauche (pl. VII, fig. 10). Lnfin, quand, dans la suite, plusieurs petits otolithes viendront s'ajouter au premier gros otolithe, ils seront déjà nombreux dans l'otocyste de droite avant de se montrer dans celui de gauche. 
J'ai parlé de cette asymétrie à propos des otolithes, mais elle se manifeste aussi dans bien d'autres organes. Elle a été signalée chez un grand nombre de Céphalophores. Malheureusement les auteurs négligent presque tous de dire quel est le côté qui se développe plus rapidement que l'autre. Dans les deux ou trois eas oì cette indication est donnée (roir p. 87-88), e’est le côté droit qui précède le côté gauehe. Et e'est tout naturel. En effel, l'enroulement de la eoquille des Céphalophores ne provient pas, comme quelques auteurs l'ont cru, de la direetion dans laquelle l'embryon tomrne dans son ouf; elle provient l'un déreloppement plus rapide du eôté qui s’enroule, et qui est, rlans la majorité des eas, le côté droit.L'enroulement est une conséquence de cette asymétrie dont l'apparition suecessive d'organes symétriques n'est qu'un cas particulier.

Mais, objectera-t-on, s'il en est ainsi, les Ptéropodes orthoconques, qui sont symétriques, devraient faire exception à la règle. Le type primitif des Ptéropodes orthoconques est bien réellement symétrique, de même que les embryons des Céphalophores en général, et l'animal adulte est symétrique aussi dans certaines parties; mais la larve ne l'est pas, comme l'enseigne un coup d'wil sur les figures 9 et 10 (pl. VIl). J'aurai à revenir plus loin sur la signification phylogénique de ces faits.

Peu après que l'otolithe a atteint le centre de la vésicule et qu'il est devenu libre de toute attache, il se met à vibrer. Les cils vibratiles qui eausent ce mouvement sont difficiles à voir. Je n'ai réussi à les distinguer que rarement et ne puis donner aucun renseignement précis sur leur disposition.

Les otocystes se trouvent, à leur origine, sur les côtés du pied, qui occupe, en ce moment; une bonne partie de la face ventrale de l'embryon (pl. VI, fig. 1, (1)). Plus tard, la base du pied se rétréeissant, les vésicules auditives sont rapprochées l'une de l'autre et viennent se placer sur les côtés de l'osophage (pl. VI, fig. $6, \omega)$, où elles entreront en rapport arec les ganglions osophagiens.

L’organe de la vision manque à la plupart des Ptéropodes; je n’aí malheureusement pas eu l'occasion de suivre l'embryogénie de Creseis, où cet organe atteint un certain degré de développement, et ne puis, en conséquence, donner aucun renseignement sur son mode de formation.

La formation du système nerveux.- Nous avons déjà vu (p. 131) que l'embryon formé présente un épaississement de l'ectoderme à la par- 
tie de la région circonscrite par le voile qui avoisine la bouche (pl. II, fig. 19, et pl. Vl, fig. 1, ne). C'est vers l'époque où le lube intestinal est à peu près formé el où la coquille va atteindre le cou de la larre, qu'a lieu, chez les Hyaléacées el les Cymbuliacées, la première différenciation de cette région; chez les Styliolacées et Clio, elle a lieu beaucoup plus tard.

Je commence par la description de cette formation chez les Hyaléacées, où elle est plus facile à observer. Le champ circonscrit par le voile a changé de forme depuis Ia fiu de la période embryonnaire; de rond qu'il ćtail, il est devenu large, et présente deux échancrul'es, l'une ventrale et l'autre dorsale (voir pl. III, fig. 30). La partie de l'ectoder'me comprise entre ces deux échancrures constitue un disque épais et provient de la région épaissie de la période précédente, laquelle se trouve déplacée par suite de l'ćchancrure du voile (pl. III, fig. 30, ne). Un enfoncement se produit lans toute la partie centrale de ce disque, excavation entourée par les bords du disque qui n’ont pas pris part à l'invagination. Ces bords constituent deux bourrelets en forme de croissants (fig. 30, ne), qui embrassent de droite et de gauche l'excavation encore peu profonde (fig. $30, \mathrm{mi}$ ), le fond des échancrures orale et aborale du bourrelet voilier achève de clore le cercle et relie entre elles les extrémités des deux croissants.

L'excavation ne conserve pas longtemps celte forme simple; elle va en s'approfondissant en deux points opposés, à sayoir : à ses extrémités latérales, au-dessous du milieu de chacun des rebords en croissant. La courbure de ces rebords augmente; ils se referment sur euxmêmes, de façon à former un 8 de chiffre couché. Les quatre cxtrémités des bourrelets circulaires viemnent donc se rencontrer au centre de la région céphalique et entourent de chaque côté une ouverture qui mène dans unc carité arrondic. En regardant de la face dorsale ou ventrale, l'on voit que les plans de ces ouvertures font un angle trèsprononcé, que les axes des excavations se rencontrent peu au-dessus de la surface céphalique. Ces deux invaginations s'approfondissent en divergeant (pl. III, fig. 32, et pl. V, fig. 6, nc). Leur's ouvertures se resserrent, le bourrelet épaissi qui entourait leurs bords prend part à l'enfoncement, el la cavité d'enfoncement se rétrécit (fig. 32, ni). Quelques cellules se détachent du fond du cecum (fig. $32^{*}$ ). Puis les parois de la double invagination s'épaississent en se resserrant, la cavité diminue et l'ouverture se referme petil à petit (pl. III, fig. 3I, et pl. V, fig. 6, ni). Les deux masses ganglionnaires. car elles ont déjà 
l'apparence de ganglions, se rapprochent alors l'une de l'autre el se soudent à leur point de contact (pl. IV, fig. 48). De bonne heure déjà (pl. III, fig. 30, ґ.n), une cellule paraît se détacher de chaque côté de la partie orale de l'invagination nerveuse; elle prend une forme ramifiće, s’insérant par de nombreux filaments à la région orale du bourrelet voilier. Ces mêmes fibres se retrouvent sur les figures 31 (pl. III) et 48 , un (pl. IV).

L'on remarquera, en comparant ces trois figures 30,31 et 48 , que les ganglions nerveux occupent un espace relativement bien moindre de la région céphalique que l'invagination à son origine. Néanmoins les ganglions sont toujours resserrés entre les denx ćchanerures du voile, parce que ees échancrures s'approfondissent et prennent de plus en plus d'importance.

Cetle paire de ganglions n'a pas un long chemin à faire pour atteindre son lieu de destination, à savoir : le côté dorsal et supérieur de l'œsophage. La bouche se trouvant dans le fond rle l'échancrure orale du voile (pl. IV, fig. 48, b), les ganglions n’ont qu’ì se détacher de la région céphalique et à descendre un peu pour arriver à lemr place définitive. Si on les regarde en ce moment par le dos de la larve, sans avoir suivi leur mode de formation, l'on peut facilement ètre tenté de croire que ces ganglions se forment à la nuque, derrière le voile. Je crois avoir suffisamment démontré qu'il en est autrement. Je dois ajouter eependant que j'ai vu parfois au sommet du dos, sous le bourrelet voilier, une rosette impaire de cellules situćes sur la ligne médiane et dont la signification m'est restée inconnue. Je n'ai vu aueun organe se développer sur ce point (pl. III, fig. 3I *).

Les ganglions céphaliques ou sus-œsoplagiens, relićs entre eux par une large soudure, viennent done se mettre à cheval sur la partie dorsale de l'osophage à son tiers an térieur. Ils se replient sur ses côtés, l'embrassent entre eux et entrent en commmnication arec les otocystes, qui occupent précisement déjà l'endroil où arrivent maintenant ces ganglions (pl. V, fig. 2, ns et (1)). Le point de soudure des deux ganglions s'étire en largeur et devient la eommissure dorsale, qui relie entre eux les deux ganglions céphaliques.

En même temps l’on distingue, immédiatement au-dessous des otocystes et du côté rentral de l'oesophage, une masse ganglionnaire transversale (pl. V, fig. 2, ni), le ganglion sous-œsophagien. D'où provient ce dernier ganglion? Je n’ai pas eneore r'ćussi à trouver son origine chez les Ptéropodes. A en juger par analogie avec d'autres 
Mollusques, il devrait se former aux dépens du mésoderme du pied. Sa forme et sa position excluent l'idée qu'il puisse provenir d'une différenciation de la masse nerreuse céphalique. Il ne peut done guère venir que de la région ventrale voisine, e'est-à-dire du pied. Il est encore entièrement séparé des ganglions sus-(esophagiens, auxquels il ne se relie que plus tarl.

Chez C'ymbulia, je n'ai pas observé pas à pas le mode de formation des ganglions nerveux. Les jemnes larres ont un épaississement de l'ectoderme de la région céplalique (pl. IX, fig. 16 et 17,ne). Chez. des larves plus àgées (pl. IX, fig. 18), l’on décourre, en les regardant d'en haut, un amas cellulaire transversal $(n s)$ qui est encore très-près de la surface et présente en somme la forme d'un croissant. Il a deux renflements latéraux, séparés sur la ligne médiane par un espace un peu plus mince. Les extrémités latérales amincies descendent sur les côtés de l'œsophage et poussent ì la rencontre des otocystes. A son bord oral se trouvent les deux tibres déjà décrites pour les Hyaléacées, et qui commencent à se ramifier (pl. IX, fig. 18, p.n). Enfin, ¿i ses extrémités latérales, se trouvent une ou deux cellules qui paraissent se détacher de sa masse et rui se ramifient à la manière des cellules museulaires $\left(\mu \cdot n^{\prime}\right)$. Un peu plus tard, cette masse descend à la reneontre de l'œesophage et prend de plus en plus la forme de deux ganglions sus-nsophagiens. L'on distingue parfois alors, en regardant l'embryon par la face ventrale, un petit amas de cellules situé au côté ventral de l'œsophage (pl. IX, figr. 19). Ces observations sont trop incomplètes pour mériter une description plus détaillée. Elles suffisent cependant à montrer que le développement des ganglions osophagiens ne peut pas différer beaucoup de celui que j’ai décrit pour les Hyaléacées.

Chez les Styliolacées et les Clioïdées, la formation du système nerveux n'a lieu que très-tard, vers la fin de la vie larvaire, qui dure ici très-longtemps. L'on ne peut done suivre ce déreloppement sur des larves élevées en captivité, et cette circonstance en rend l'étude trèsdifficile. La différenciation ultérieure de l'anneau osophagien tombant en dehors de la vie larvaire, j'en renvoie la deseription à la période suivante.

La formation des muscles. - J'ai déjà parlé du dévoloppement des fibres musculaires isolées du voile, du bord du manteau, du muscle triturant de l'estomac; il ne me reste qu'à parler de la formation des muscles rétracteurs. 
Il n'y a qu'un seul muscle rétracteur ou columellaire chez tous les Thécosomes que j’ai observés. Il m'a semblé qu'il prenait naissance d'un amas de cellules détachées de l'ectoderme et situé du côté dorsal de l'endroit où se trouvait l'invagination préconchylienne; cet amas s'allonge et m'a paru donner naissance au muscle columellaire, qui serait donc pluricellulaire dès son origine. Toutefois, n'ayant pas fait une étude spéciale et suivie de ce point, je n’insiste pas.

Le moment où apparaît ce muscle coïncide généralement avec celui où le voile commence à s'élargir. Il s'attache un peu au-dessus de la pointe de la cơfuille, et généralement un peu vers le côté droit. II se bifurque à peu près à la hauteur de l'estomac; ses deux branches, prenant entre elles l'œsophage et l'anneau nerveux orsophagien, vont se ramifier de part et d'autre dans l'extrémité dorsale de la base du pied (pl. III, fig. 39 ; pl. V, fig. 2, et pl. VI, fig. 6 et 7 , $\mu r$ et $\mu r /$ ).

Chez Clio, le musele rétracteur columellaire occupe la même position que chez les Thécosomes. Il est également bifurqué (pl. X, fig. ‘ et $8, \mu r l)$, et je me suis assuré par l'emploi de l'acide acétique que chacune de ses branches ne représente à l'origine qu'une seule cellule hišlologique. Chaque branche renferme, en effet, un grand nucléus ovale (pl. $\mathrm{X}$, fig. 8 , v), et la partie inférieure, non divisée, du muscle, paraît n'être que le résultat de la fusion partielle de ces deux cellules. Cie muscle s'insère d'abord au bourrelet coquillier et au bourrelet moteur du voile. Plus tard, ces points d'insertion se modifient.

Mais, outre ce nuscle, qui répond au muscle unique des Thécosomes, Clio possède un second muscle que Krohn (roir p. 75) a déjà observé chez quelques larves de Gymnosomes. Ce muscle prend son origine près de l'extrémité de la coquille, mais du côté ventral, visà-vis du précédent (pl. X, fig. $\left.7, \mu^{\prime} v^{\prime}\right)$. Il est plus mince que le muscle columellaire et non hifurqué. Il remonte du côté gauche de l'estomac, passant entre les deux sacs nutritifs, et va se perdre vers la base du pied. Son origine se trouve, comme pour le muscle columellaire, dans des cellules détachées, du côté interne de l'ectoderme, et qui s'allongent. Une seule cellule constitue ce muscle rétracteur rentral à son origine. La présence de ce second muscle rétracteur est, sans doute, en relation avec la position des nageoires chez les Gymmosomes.

Que la bande musculaire provienne d'une ou de plusieurs cellules. tuujours est-il que chez les Thécosomes comme chez les Gymnosomes les noyaux de ces cellules disparaissent et que chaque bande n'est bientôt 
plus qu'un faisceau de fibres cylindriques, homogènes, assez réfringentes. Chaque faisceau s'est enveloppé d'un périmysium.

Je reviendrai sur la terminaison supérieure de ces muscles en parlant du développement des nageoires.

La formation du cour et de la glande rénale. - Ces deux organes sont trop intimement liés pendant leur déreloppement chez tous les Céphalophores, pour que je les traite séparément. Le rein étant, le premier à se former, c'est par lui que je commencerai.

Nous avons vu (p. 141) qu'au point où commence, chez les Hyaléacées, l'enfoncement de la eavité palléale, du côté droil, à la base du roile, se trouve un épaississement local de la couche ectodermique: Cet épaississement est entrainé par l'enfoneement, au bord inférieur duquel on continue à le trouver. La cavité palléale s'approfondissant, surtout du côté dorsal, le petit épaississement se trouve repoussé et rapproché de la face ventrale. Ce n'est qu'au moment où le repli palléal dépasse déjà en arrière le niveau de l'estomac, par conséquent chez des larves entièrement formées, que commence la différenciation de cet amas cellulaire. Il est, en ce moment, de forme allongée, cylindrique et compacte; son grand axe est dirigé transversalement. Celle de ses deux extrémités qui est la plus voisine de la ligne médiane ventrale, se létache petit à petit. A ce même moment, l'on commence à discerner, au milieu du tissu mésodermique qui s'interpose entre la masse nutritive el l'ectoderme qui tapisse la coquille, un amas ovale de cellules rondes. Cet amas, qui n'est autre que l'origine du cœur, est situé au milieu de la face ventrale, à quelque distance au-dessous de l'anus (pl. IIl, fig. 37, h). L'extrémité de l'amas cellulaire rénal, se détachant du repli palléal ( $\mathrm{pl}$. 111, fig. $37, r$ ), se trouve de suite en contact arec ce rudinent du cœur. Bientôt les deux amas se ereusent dans leur longueur' l'amas rénal se ereuse le premier d'un fin canal qui débouche, en formant un coude, dans la earité palléale (fig. 37, $r c)$. Ce canal est encore fermé à son extrémilé interne qui touche au cœur. Ce dernier, s'étant creusé, se montre sous la forme d'une résicule ovale, dont les parois sont composées d'un épithèle simple (pl. III, fig. 37, h). La eavité du cœur est allongée, mais étroite ; elle s'élargit rapidement aux deux bouts et s'étrangle au milieu. Au lieu d'une résicule unique ovale, à parois épaisses, nous trourons done bientôt deux vésicules accolées, spacieuses, à parois minces. Les cellules, de cylindriques qu'elles étaient, sont devenues lenticulaires (pl. V, fig. 丂). La vésicule gauche (qui se trouve à droite sur la figure) 
est l'origine du ventricule (fig. $5, h v$ ); celle de droite (ho) n'est autre que l'oreillette; elle se distingue déjà par la présence de quelques cellules contractiles étoilées, qui s'attachent aux parois opposées. Ces cellules iront en se multipliant plus tard. Le ventricule n'a pas de cellules de ce genre et ses battements sont uniquement dus à la contractilité des cellules aplaties de sa paroi, qui se différencieront plus tard en deux couches et formeront un système de fibres musculaires en forme de cercles. Une ouverture qui se ferme et s'ouvre alternativement à chaque contraction fait communiquer entre elles les deux chambres. Je n'ai pu découvrir eneore à cette époque de réritable valvule. Le cocur bat déjà, quoique d'une manière intermittente et irrégulière.

Pendant que le cour se déreloppait ainsi, le rein n'est pas resté en arrière; son canal a percé la paroi contiguë au cœur (pl. V, fig. š, roh), formant ici aussi un angle droit avec la partie moyenne dı eanal. Un point d'attache relie l'cxtrémité interne du rein à l'ectoderme.

En examinant attentivement les rapports de l'ouverture interne avec le cour, l'on s'aperçoit qu'elle ne donne pas directement dans la cavité de cet organe. Elle s'ouvre tout contre la paroi de ce dernier, mais au côté externe de cette paroi. Les bords de l'ouverture sont évasés en entonnoir et s'attachent par de minces fils à cette face externe (pl. V, fig. 5̌, roh). De péricarde, il n'y a pas encore de trace; mais, dès qu'il se formera par la condensation des tissus qui entourent le cour, le canal rénal s'ouvrira dans la cavité péricardienne.

Les deux ouvertures du canal rénal sont munies de cils vibratiles, courts, mais très-distincts et très-actifs. Ils sont encore plus courts dans l'intérieur du canal rénal, qu'ils tapissent dans toute sa longueur.

La position relative du cœur et du rein rarie beaucoup suivant les genres. Les organes sont toujours contigus; mais, tandis que chez Hyalocylis striata, qui a, jusqu'à présent, principalement servi de base à ma description, le cœur se troure au-dessous du rein et légèrement à gauche (pl. V, fig. 2, r et $h$ ), chez Cavolinia tridentata il se troure au mème niveau, du côté gauche (pl. III, fig. 37, $r$ et $h$ ), et vient ensuite se placer obliquement au-dessus du rein (pl. III, fig. 38, r et $h$ ). Dans ce cas, l'ouverture interne $d u$ rein se trouve vis-à-vis du bord latéral de l'oreillette, au lieu d'être vis-à-vis du bord supérieur du ventricule. Ces modifications de position relative sont sans importance.

Je n'ai pu sujvre en détail la formation de ces organes chez les 
autres ptéropodes, à cause de l'ẻpoque tardive à laquelle ils apparaissent ehez ces genres à période larvaire prolongée; mais les quelques larves que j'ai pèchées en mer me donnent à penser que le mode de formation ne duit pas différer beaucoup ici de celui que j’ai décrit pour les Hyaléacées.

Les modifieations ultérieures du cœur et la formation des vaisseaux sanguins tombent en dehors de la période larvaire.

Nous avous passé successivement en revue le développement de tous les organes dont la larve est munie avant sa transformation. Avant de passer à la période suivante, jetons encore un coup d'œil sur les différences que présentent les divers types sous le rapport de la rapidité et de l'ordre de succession dı déreloppement de ces organes.

Chez les Hyaléacées, la larve se développe rapidement, les organes larvaires atteignent peu d'extension et les organes défnitifs se déve: Ioppent de bome heure. Ainsi, le voile reste relativement petit, le tube intestinal, la cavité palléale, les muscles, les nageoires, le système nervenx, le cœur et le rein se sont formés dans l'espace d'une semaine(aux mois de mai el de juin) el la larve subira sa métamorphose au moment où sa coquille dépasse à peine la limite de la coquille larvaire (pl. Ilt, fig. 38 et 39 , et pl. V, fig. 2).

Chez les Styliolacées, la larve a bien atteint, au bout de huit jours (dans la même saison), la même longueur proportionnelle(pl.VI, fig. 6), mais la cavité palléale commence à peine à être.indiquée; le pied est eneore simplement arrondi, le voile encore peu développé; le cœur, le rein, le système nerveux manquent encore entièrement, et le tube intestinal est seul à peu près formé. Chez des larves bien plưs âgées (pl. VI, fig. 7), le voile a atteint de grandes dimensions, la coquille a continué à croître, mais le pied commence à peine à se lober, la cavité palléale et la masse nerveuse sus-œsophagienne commencent à peine à s'iuvaginer. Dans l'estomac se forme un organe de trituration larvaire rqui devra tomber plus tard pour faire place à des dents pareilles à celles qui se forment dès l'abord chez Hyalcea. En d'autres termes, la larve, en tant que larve, atteint ici un développement plus caractérisé et vit plus longtemps dans cet état.

Les Cymbuliacées et les Clioïdées paraissent oceuper, sous ce rapport, une position intermédiaire entre ces deux extrêmes.

Ce sont là de simples différences physiologiques, des différences d'adaptation, qui n’affectent pas les procédés mêmes du développement. 
Toutes ees larves nagent à l'aide de leur voile, la tète en haut, la coquille en bas. Elles nagent un moment, montant jusqu'à la surface de l'eau, puis rentrent leur voile dans leur coguille et se laissent tomber de quelques centimètres, pour recommencer aussitôt à nager. Les larves de Cavolinia, et peut-être aussi celles des autres espèces, sont sensibles à la lumière ; elles la fuient. Dans mon hocal contenant un banc de larves (c'est le mot, car ces larres se tiennent ensemble comme un banc de poisson), l'on remarque qu'elles se réunissent Loujours du côté opposé à celui d'où vient la lumière. Elles fuient également la lumière du jour et celle d'une lampe. En déplaçant la lampe, on peut faire déplacer le banc de larves à volonté. Ce déplacement n'est pas rapide.

Cette sensibilité à la lumière est singulière en l'absence de tout organe visuel. Je me suis tout naturellement demandé d'abord si ee n’était pas la chaleur émanant du point lumineux et non la lumière que percevaient les larves. Mais, en employant un mode d'éclairage qui n'est accompagné que d'un faible rayonnement ealorique, l'effet est le même. L'on peut même, en faisant tomber d'un côté la lumière et en plaçant de l'autre une source beaucoup plus puissante de chaleur obscure, s'assurer que c'est bien la lumière et non la chaleur qu'elles fuient. En y réfléchissant, cependant, l'on ne voit pas pourquoi, chez des animaux peu différenciés, le système nerveux tout eutier ne serait pas capable de percevoir la lumière. Chez les animaux supérieurs, cette fonction est localisée dans une certaine partie du système nerveux: la rétine. Mais de ce que la fonction n'est pas localisée chez des êtres plus inférieurs, il serait imprudent de conclure qu'elle n'existe pas. Elle peut être généralisée tout en étant moins parfaite. La lenteur avec laquelle les larves cheminent dans la direction opposée à celle d'où leur viennent les rayons lumineux semble bien indiquer qu'elles n'ont qu'une perception peu nette de la direction de ces rayons.

L'observation que je rapporte est encore intéressante en ce qu'elle nous montre où doit avoir lieu le développement de ces larves dans la nature : c'est au fond de la mer. Ainsi s'explique l'extrême rareté des larves d’Hyaléacées parmi les animaux pêchés à la surface. 


\section{LA MÉTAMORPHOSE ET LE DÉVELOPPEMENT ULTÉRIEUR.}

Je n'ai observé directement la métamorphose que chez les Hyaléacées et me bornerai donc à la décrire pour cette tribu, en intercalant quelques observations éparses sur d'autres tribus.

f.e changement le plus apparent et aussi le plus important est celui des organes de la locomotion. Le roile commence à se réduire, à se ratatiner, et, en même temps, les lobes latéraux du pied croissent rapidement en longueur et en largeur. Tes cellules du bourrelet voilier se détachent avec les cils moteurs, qui continuent encore longtemps à battre l'eau d'un mouvement automatique et à promener en tous sens les cellules qui les portent. Enfin il ne reste plus que de petites houppes ciliaires attachées à la nuque, et celles-ci tombent à leur tour.

Pour quiconque a observé cette chute du voile, il ne peut y avoir le moindre doute que le voile ne soit un organe purement larvaire, dont aucune portion ne persiste après la métamorphose. Sa position, lorsqu'il est déjà suffisamment réduit, est au-dessous de la base des nageoires; il ne peut donc contribuer à leur formation, et les lignes ciliailes de la base des nageoires ne s'observent que beancoup plus tard et n’ont rien de commun avec lui. J. Muiller a déjà démontré cela (XCIII) en faisant voir que ces lignes ciliaires se trouvent à la face ventrale et non à la face dorsale des nageoires. Les bords postérieurs de ces organes prennent la bouche entre eux et de dorsaux deviennent médians; le pied s'élargissant, le bord antérieur de ces nageoires devient externe, et d'antéro-postérieur leur plan devient latéral (comparer pl. V, fig. 2 et 3 , et pl. IV, fig. 43).

A mesure que les nageoires s'allongent, l'on voit se former dans leur intérieur un système très-régulier de fibres musculaires. Nous avons ru que déjà pendanı la vie larvaire, des cellules s'étaient accumulées à leur partie supérieure (pl. V, fig. 2, ı.c), et nous les avons vues s'allonger dans le sens de l'ćpaissem de la nageoire et relier entre elles les deux parois opposées de cet organe. Ces cellules musculaires vont en se multipliant beaucoup, tandis que l'amas cellulaire qui leur donne naissance persiste encore longtemps vers l'extrémité et sur les bords de la nageoire (pl. V. fig. 3. et pl. IV. fig. 43, «c). Les 
fibres auxquelles elles donnent naissance sont d'abord rentlées au milieu, plus tard cylindriques, et s'attachent de part et d'autre à l'ectoderme par leurs extrémités, ramifiées en plusieurs fibrilles. En regardant la nageoire de profil, ces fibres se distinguent aisément (pl. IV, fig. 44, «ps); vue par sa surface large, la nageoire montre des séries de petits points qui vont en augmentant de nombre vers l'extrémité supérieure (pl. V, fig. 3, r.ps) et ne sont que la coupe optique de ces fibres. En ajustant le microscope au niveau de l'épiderme, l'on voit, au lieu de petits points, de petites étoiles qui répondent aux insertions ramifiées de chaque fibre.

Presque en même temps se développe un système de fibres musculaires longitudinales, dont les unes se dirigent obliquement de bas en haut et de dedans en dehors, tandis que les autres ront de bas en haut et de dehors en dedans (pl. IV, fig. 43, et pl. V, fig. 3, : $: p l$ ). Cies dernières règnent à la face dorsale de la nageoire, tandis que le premier système en occupe la face ventrale.

Ces fibres sont assez larges et épaisses (־ millièmes de millimètre environ), homogènes et à bords parallèles, sauf vers le sommet de la nageoire, où elles vont en se perdant. Les fibres de chaque système sont parfaitement parallèles entre elles; les deux systèmes se croisent à angles aigus, produisant un dessin losangé parfaitement régulier. Ces fibres m'ont paru se développer aux dépens de la couche ectodermique, qui se dédouble en un épiderme cellulaire et un mésoderme musculaire.

En dernier lieu se forme une série de fibres transversales (pl.IV, fig. 43, et pl. $\mathrm{V}$, fig. 3, r.pt) qui ont dans l'origine un renflement nucléé à leur milieu. Elles s'attachent aux deux bords de la nageoire, sont parallèles entre elles, mais peu nombreuses et très-minces. Ces dernières fibres n’acquièrent pas une plus grande importance par la suite, tandis que les trois premiers systèmes de muscles prennent un grand développement.

Le lobe médian du pied est constitué comme les nageoires, sauf que les fibres transversales lui font défaut et que les fibres longitudinales ne se croisent pas et ne forment pas le mème dessin régulier que dans les nageoires (pl. IV, fig. 44, p).

Une partie des fibres musculaires longitudinales, tant de la nageoire que dupied, entrent en communication arec le muscle columellaire. Celui-ci se divise à sa partie supérieure en trois faisceaux, dont deux latéraux (pl. IV, fig. 43, $\left.\mu . r^{l}\right)$ et un médian ( $\left(\mu r^{\prime} m\right)$. Ce dernier se 
détache du laisceau latéral de droite, passe au côté dorsal du cou el se divise au niveau de l'anneau nerveux cesophagien en une foule de fibres qui sont en continuité avec celles de la couche musculaire dorsale de la base de la nageoire. Les faisceaux latéraux se terminent dans les systèmes de fibres musculaires du pied el de la partie externe et ventrale des nageoires (pl. IV, fig. $\mathbf{4 4}, p . r l$ ).

Chez Iy yalocylis striata, le muscle columellaire ne se divise qu'en denx laisceaux latéraux ( $\mathrm{pl} . \mathrm{V}$, fig. $3, \mathrm{wr} l$ ), qui ne se soudent que peu avec la musculature propre de la nageoire et se ramifient jusque près du bout de celle-ci. Plus tard, ce muscle prend un plus grand déreloppement (pl. V, fig. $4,(\mu r l)$. On le retrouve aussi chez des jeunes assez avancés de Cavolinia (pl. IV, fig. 46).

De l'angle supérieur des ganglions céphaliques part de chaque côté un gros nerf qui se distribue bientôt aux muscles de la nageoire et du pied (pl. V, fig. $3, n n$ ). Suivre le mode de formation de ce nerf ne serait pas chose difficile; j’ai malheureusement négligé de le faire.

Le bord libre du pied et des nageoires continue à être garni tout le tour d'une rangée de cirrhes fins et immobiles (pl. IV, fig. 43, et pl. V, fig. $3, c r p)$; trois ou quatre cirrhes raides très-forts et très-longs sont implantés perpendiculairement au milieu de la face dorsale de chaque nageoire chez Cavolinia (pl.1V, fig. 44, cr). Les bords internes des nageoires ont une tendance à se rapprocher l'uii de l'autre par la base, et la bouche se porte un peu en arant de façon à prendre une position plus ou moins centrale cutre les nageoires et le pied (pl. IV, fig. 43 et $44, b$ ).

La bouche forme une saillie conique, couverte de cils vibratiles. Cette toison ciliaire s'étend sur une partie de la face supérieure du lobe médian du pied, ainsi que sur la face interne de la base des nageoires.

La radule est déjà bien formée et ne diffẻre de celle de l'adulte que par le nombre de rangées de dents. Elle se développe dans le cæcum, dont nous avons vu la formation (p. 144).

Nous avons vu des replis longitudinaux épais se former à la paroi interne de l'estomac. L'on ne roit d'abord ifue trois de ces replis, puis quatre; ils ont une base large et une forme triédrique. Bientôt apjrès leur apparition, ils commencent à se coutrir d'une couche cornce qui prend exactcment la forme de la saillie sur laquelle elle naît. II se forme alor's encore une cinquième saillie qui se courre à sõn tour de substance comée; áinsi naissent les cinq dents stomácales (pl.IV, 
fig. 43; pl. V, fig. 3, et pl. VI, fig. 8, d). Ces dents ont été observées chez tous les Thécosomes à l'état adulte.

Au-dessus de chaque dent se trouve un repli de la muqueuse, moins prononcé que celui qui donne naissance à la dent et qui ne se recouvre pas d'une plaque cornẹe ( $\mathrm{pl}$. V, fig. 3 , et pl. VI, fig. 8, sp).

Nous avons vu une couche extérieure distincte se séparer de la paroi de l'estomac et se transformer en un système de muscles circulaires. Ce muscle triturant acquiert une assez grande puissance à mesure que les dents se développent (pl. IV, fig. $43 ; \mathrm{pl}$. V, fig. 3 , et pl. VI, fig. $8, \mu . s$ ). 11 occupe une zone correspondant à la longueur des dents; les fibres sont rangées au milieu sur trois d'épaisseur; vers les bords de la zone elles sont sur deux, puis sur un rang. Leur action est de resserrer les dents, d'abord à la partie supérieure, en sorte que les surfaces dentaires se touchent par toute l'étendue qui est au-dessus de la pointe de la dent. Les fibres inférieures se contractant à leur tour, chaque dent' exécute un mouvement de bascule et s'applique contre les autres par les surfaces situées au-dessous de la pointe. Puis les fibres se relàchent, les dents s'écartent les unes des autres pour recommencer bientôt la même série de mourements. C'est un puissant appareil de mastication. Le rôle dles replis au-dessus des dents paraît être seulement de repousser les particules de nourriture entre ces dernières.

Le sac nutritif débouche dans l'estomac, tantôt (Hyaléacées) près du pylore ou dans le pylore même, du côté dorsal, tantôt (Styliolacées, Créséidées) dans l'estomac, dans une région plus ou moins éloignée du pylore, mais toujourşplus près de la face dorsale que de la face ventrale ( $p l$. VI, fig. 5,6 et $7, \sigma)$. Le canal excréteur $(\sigma c)$ est son seul point d'attache.

Il est très-probable que, chez les genres à état larvaire prolongé, l'estomac se déplace, ainsi que le fait observer Krohn (p. 48), et que l'estomac de l'adulte se forme en majeure partie aux dépens de l'œsophage de la larve.

Nous avons quitté le sar nourricier au moment où il commençait à changer d'aspect, où la matière nutritive était en grande partie résorbée et où ses parois commençaient à s'amincir. Des goultes de graisse, pareilles à celles que l'on trouve déjà dans le vitellus en segmentation, se détachaient des parois. mêlées à de la substance nutritive. Elles étaient chassées du sac nourricier, par les mouvements péristaltiques de cet organe, dans l'estomac, qui les chassait à son 
tour dans le sac nourricier, et ce mourement deva-et-rient durait assez longtemps avant que les masses fussent mastiquées el digérées. Ces mourements sont causés pas la contraction successive des filjres musculaires qui entourent le sac en manière de cercles. A cette époque aussi, le sac nutritif commence à jaunir. Ce changement est peu appréciable chez la plupart des espèces, où la matière nutritive est naturellement jaune; mais, chez Cavolinia, où elle est rouge, l'on roit clairement le sac prendre une teinte de plus en plus jaune, qui contraste avec la coloration rouge de plus en plus marquée de la pean (pl. IV, fig. 43 et 44 ).

Le sori ultérieur du sac, nutritif rarie énormément suivant les genres. Chez les Hyaléacées, il diminue rapidement à mesure que le foie se développe, et il n’en reste bientôt plus qu'une petite masse arrondie ou lobée, située à l'extrémité postérieure du corps, et autour de laquelle se développera la glande génitale ; cette vésicule communique toujours avec le pylore par un fin canal. Eydoux et Souleyet l'ont parfaitement décrite, mais la prennent, à tort selon moi, pour une vésicule biliaire.

Chez les Styliolacées et les Créséidées, le foie n’apparaît que trèstard et le sac vitellin ne diminue que lentement. Même chez des individus jeunes qui ont atteint la moitié de la dimension de l'adulte, le foie n'est encore que rudimentaire et les parois du sac vitellin présentent dans ce cas une particularité remarquable. Les cellules de cette paroi se chargent de granulations jaunes (pl. Vl, fig. 4 et 8,, ) que l'on retrouve dans la cavité du sac et dans l'estomac. Cette constitution s'observe chez de jeunes exemplaires de Gréséis, même lorsque le foie a atteint déjà un certain développement. Mais bientôt après le sac tombe en dégénérescence et la glande sexuelle se développe à sa face externe. Faut-il en conclure (que la matière nutritive ne s’absorbe chez ces genres que très-lentement et que les grains jaunes ne sont qu'un produit de la dégénérescence ? L'agrandissement notable du sac nutritif, après la résorption de la substance nutritive qu'il contenail, s'oppose à cette manière de voir. Ainsi les figures 5 et 7 (pl. VI) représentent des larves dont la provision nutritive est près de s’épuiser. Les figures 4 et 8 montrent des jeunes des mêmes espèces où le sac nutritif a pris un développement très-frappant. Cette croissance du sac nutrilif, chez certains genres, après que la substance nutritive en a disparu, montre clairement qu'il joue ici temporairement un iutre rôle que celui de simple réservoir de nourriture. 
Faut-il penser que le sac nutritif remplit provisoirement le rôle de foie jusqu’à ce que le foie soit assez développé pour sécréter la bile en quantité suffisante? Cette dernière alternative est infiniment plus plausible, d'autant plus que, chez les Creseis, les cæecums hépatiques naissent, non pas seulement sur la paroi de l'estomac, mais encore sur le canal efférent du sac nourricier.

En tous cas, si le sac nutritif peut jouer chez certains genres le rôle de foie, ce n'est qu'un organe hépatique prorisoire. Chez les Hyaléacées, il se résorbe purement et simpleınent et ne remplit jamais d'autre fonction que celle de réserroir de nourriture.

Ce sac nutritif de Creseis et de Styliola a déjà été vu par divers auteurs (voir p. 44 et suiv), mais aucun n’avait suivi arec précision son mode de déreloppement ni son sort ultérieur.

Le foie ne fait son apparition, chez les Hyaléacées, que tout à la fin de la vie larvaire (Hyalocylis striata), ou après la métamorphose (Cavolinia). Chez les autres Orthoconques, il se développe plus tard encore. Le premier rudiment du foie est un petit mamelon que forme la paroi de l'eștomac du côté ventral et tout près du pylore. Ce mamelon se change en un petit diverticule (pl. V, fig. 2, f); un second apparaît bientôt à côté et à gauche du premier (pl. IV, fig. $44, f)$, se formant par le même procédé d'évagination de la paroi stomacale. Les ouvertures de ces deux petits cæcums se confondent en une seule. Les cæcums s'agrandissent et d'autres viennent s'ajouter à l'entour, formant petit'à petit cette grosse masse framboisée, d’un brun verdâtre, qui entoure le pylore et l'origine de l'intestin des Orthoconques adultes, et que personne n'hésite à considérer comme le foie (pl. IV, fig. 46, et pl. VI, fig. 3 et $4, f)$. L'intestin, qui ne faisait d'abord qu'une anse simple ver's le bas (pl.IV, fig. 43, i), se met à s'allonger. L'anse devient plus grande et tourne sur elle-mème de façon à former une boucle. Au sortir de l'estomac, l'intestin se dirige d'abord en bas et à gauche, puis tourne de gauche à droite et remonte ensuite vers l'anus en croisant en avant la première partie de l'intestin (pl. Vl, fig. 4 et 8 , $i$ et $a$ ). L'intestin est mince; son canal est très-fin et cilié. Le foie est la seule glande qui y débouche.

Nous avons déjà vu le cœur et le rein suffisamment formés pour n’avoir plus qu'à grandir et à prendre leur position définitive, position qui varie suivant les genres. Le rein, cependant, étail encore massif et percé d'un fin canal; il grandit et devient une grande vésicule simple (Creseis) ou muni d'une foule de crecums à parois minces et 
cellulaires. Ces cellules se chargent de concrétions jaunes et tout l'organe prend une teinte jaunâtre. Il bat, à l'origine, avee autant de vivacité que le cour, mais d'une manière moins régulière.

L'aorte (pl. IV, fig. 4ŏ, ha) se forme de même que le cœur, par une condensation de eellules mésodermiques, qui forment d'ahord un cordon mal défini, puis un canal qui communique avec l'extrémité du ventricule. Les parois de ce vaisseau ne sont d'abord formées que d'une couche de cellules plates; sur une coupe optique, chaque paroi se présente comme une série de petits traits dont chacun est le nucléus d'une des cellules épithéliales, vu de profil. Au sortir du cœur, l'aorte s'infléchit d'abord en bas, contournant le fond de la eavité palléale, puis remonte au côté droit jusqu'au niveau de l'anneau œsophagien, où elle se perd. Plus tard on peut suivre ses ramifications jusque dans les nágeoires, ainsi que l'a fort bien décrit Gegenbaur. La branche viscérale de l'aorte se forme plus tard que la branche céphalique.

Le péricarde est reconnaissable chez de tout jeunes individus, mais son développement est très-lent; à mesure qu'il se développe, on roit de plus en plus nettement que e'est la cavité du péricarde, et non celle dlu cœur, qui communique avec le canal rénal.

Nous avons"quitté l'anneau œsophagien au moment où il se composait de deux ganglions sus-œsophagiens $(n s)$, reliés entre eux par une commissure dorsale, et d'une masse ganglionnaire sous-œsophagienne (ni) à peine bilobée. Ces deux masses nerveuses ne communiquaient pas encore l'une avec l'autre; les otocystes se trouvaient entre les deux (pl. V, fig. 3, ni et ns). Jues commissures latérales s'établissent maintenant par soudure, en 'arrière et au-dessous des otocystes ( $\mathrm{pl}$. IV, fig. 43, ncl). Les otocystes restent dans l'angle que forme l'anneau cesophagien, en contact aussi bien avec la masse sous-ocsophagienne qu'avec la masse céphalique (pl.IV, fig. 44, $\omega$ ); et e'est cependant avec cette dernière seulement 'qu'elle entrera en relation plus intime, ainsi que l'a montré de Lacaze-Duthiers.

Les modifications ultérieures de l'anneau cesophagien consistent en un élargissement des ganglions céphaliques vers le côté ventral, au-dessus des otocystes. Ils forment ainsi presque un second anneau wsophagien supérieur et séparé de l'annean inférieur par une échancrure où se trouvent les otocystes. Puis ces ganglions céphaliques se divisent en quatre, dont deux sur les côtés de l'œsophage (pl. V, fig. 7 , et pl. VI, lig. $8, u s^{\prime}$ ) et deux du côté dorsal (ns). Le ganglion 
sous-œsophagien (ni) ne change pas de position. Deux petites masses (pl. V, fig. 7, et pl. VI, fig. $8, n v$ ) deviennent visibles sur les côtés de l'œsophage, juste au-dessous du sac lingual, et recourertes en partie par les ganglions céphaliques. Ce sont sans doute les ganglions viscéraux découverts par van Beneden et vus aussi par Gegenbaur. Leur mode de formation m'a échappé.

Un gros nerf part de chaque còlé du ganglion pédieux, se ramifiant dans le manteau. Le nerf du côté droit se divise en deux grosses branches, dont l'une se rend au manteau, tandis que l'autre vient se perdre dans le bourrelet vibratile de l'entrée de la carité palléale (pl. IV, fig. 43 et $44 ;$ pl. V, fig. 3 , et pl. VI, fig. 8 , mx). Une telle richesse nerveuse pour un si petit organe, uniquement composé de cellules épithéliales vibratiles, mène naturellement à la conclusion qu'il s'agit ici d'un organe des sens, soit d'un organe olfactif, soit d'un organe du toucher. A en juger par analogie arec les Hétéropodes, c'est la première alternative qui paraît la plus proJable.

Les otocystes ne subissent d'autres modifications qu'une croissance et un amincissement proportionnels de leurs parois. Le gros otolithe de la larve subsiste tonjours; seulement une quantité d'otolithes plus petits et ovales viennent l'entourer et former autour de lui une couche continue (pl. VI, fig. 8, $\omega$ ). Ils le couvrent de toutes parts et il faut un peu d'attention pour s'assurer que le centre de cel amas est occupé, non pas par de pelits otolithes pareils à ceux de la surface, mais par le gros otolithe larvaire. Les petits otolitlies se forment plus tôt du côté droit que du côtẻ gauche, el l'on en voit sourent en assez grand nombre dans l'otocyste de droite, tandis que le gros otolithe vibre encore tout seul dans l'otocyste de gauche (pl. V, fig. $7, \omega)$.

Les larves possèdent, comme nous l'avons vu, un organe de circulation larvaire, situé du côté dorsal, dans la partie recouverte par le manteau. Cet organe n'est autre chose, chez les Hyaléacées, que la paroi qui sépare le corps de la cavité palléale, et qui est munie de fibres musculaires transversales (pl. IV, fig. 43, et pl. V, fig. 3, kn). Chez Creseis, une réritahle vésicule contractile se différencie à la partie supérieure de cette paroi, par un développement plus grand de ses éléments musculaires ( $\mathrm{pl}$. VI, fig. 5 , lin). Dans les deux cas, ce sinus contractile continue à fonctionner assez longtemps après la métamorphose et à une époque où le cour et l'arte sont déjà formés. Beaucoup plus tard, lorsque celte membrane a complétement cessé ses contractions ondulantes, elle est encore susceptible de se dilater 
el de se resserrer par moments; ces fibres musculaires transversales se retrouvant jusque chez l'adulte. J. Müller a déjà décrit ce sinus (voir p. 78).

La plaque vibratile du manteau acquiert un développement plus grand et ses cellules s'accentuent mieux; le hourrelet vibratile de l'entrée de la cavité palléale persiste sans prendre un accroissement proportionnel. Des lignes transversales ciliaires se forment surtout vers l’entrée de la cavité palléale. Chez certains genres (Hyaléacées), une branchie très-simple se forme au fond de la cavité palléale par un simple plissement de sa paroi. Chez d'autres genres (Creseis, Spirialis), les petits mamelons ou crêtes, couverts chacun d'une rangée de cils vibratiles, se multiplient et produisent des courants d'eau trèsvifs dans cette cavité. Le bord du manteau dépasse souvent le bord de la coquille du côté gauche et forme un prolongement cylindrique convert de cils viloratiles (pl. IV, fig. 43,44 et $46, m f$ ).

La croissance ultérieure de la coquille mérite une attention toute particulière, puisque c'est en m'appuyant sur les différences que présente ce mode de croissance que j’espère réussir à jeter les bases d'une classification naturelle des Thécosomes, surtout de la tribu des Orthoconques, dont la systématique est, jusqu’à présent, fort embrouillée.

Nous avons vu que la coquille croît suivant une certaine loi, dans une certaine forme, jusqu'à ce qu'elle ait atteint le cou de la larve ; là il se forme chez les Orthoconques une ligne circulaire bien marquée, et chez les Gyınnosomes ?un anneau ou bourrelet encore plus facile à voir. Puis la coquille se met à croître sur un modèle trèsdifférent. Je donne à cette première partie de la coquille le nom de coquille larvaire.

Chez les IIyaléacées, cette coquille a une forme de cornet, arrondi au sommet et un peu rétréci à la limite supérieure (pl. IV, fig. 43 et $44, q t)$. Elle est marquée de lignes circulaires transversales trèsaccentuées et très-régulières chez tous les genreș observés, à l'exception de Hyalocylis. Vue de face, cette coquille est symétrique; mais, vne de profil (pl. IV, fig. 45), elle présente une courbure très-marquée à concavité dorsale. Elle est aplatie d'avant en arrière et étirée par les côtés, qui remontent un peu plus haut que la partie médiane du bord.

La cocguille larvaire de Hyalocylis striata a la forme d'un dé à coudre; elle est lisse et presque droite, même lorsqu'on la regarde de profil (pl. V, fig. 2 et 3). 
La coquille définitive des Hyaléacées a un angle d'ouverture beaucoup plus grand (pl. IV, fig. 46, qd). Chez Cavolinia, les stries sont remplacées par des anneaux alternativement un peu plus et un peu moins larges, formant de profil une ligne ondulée. Vue de profil, la base de la coquille définitive montre encore la même courbure à convexité ventrale que la coquille larvaire, en sorle que les deux extrémités sont dirigées à 90 degrés l'une de l'autre. L’animal quitte sa coquille larvaire petit à petit et se retire dans la partie supérieure, qu'il ferme en bas par une cloison horizontale. Cette cloison se trouve un peu au-dessus de la limite des deux portions de la coquille. Le point d'insertion inférieur du muscle rétracteur est remonté petit à petit et se trouve au côté dorsal, juste au-dessus du point d'insertion du diaphragme mentionné. Puis la coquille larvaire se détache par fracture, et il est très-rare qu'on la rencontre encore chez des jeunes aussi avancés que celui de la figure 46. Cette fracture n'est donc pas un accident; c'est la r’ègle, comme l'indique assez la formation du diaphragme pendant que la coquille larvairc est encore entière.

Hyalocylis striata présente la même série de changements que Cavolinia. Seulement la coquille larvaire ne forme pas un angle aussi prononcé avec la coquille définitive et se casse par conséquent moins facilement. Il n'est pas rare de rencontrer des jeunes comme celui de la figure 4 (pl. V), possédant encore cette coquille; on ne la retrouve plus sur des individus plus âgés, mais les bords fracturés qui dépassent le diaphragme se trouvent jusque chez l'adulte.

Chez Hyalcea, la coquille larvaire, recourbée et striée comme celle de Cavolinia, persiste jusque chez l'adulte, où elle est encore occupée par l'extrémité postérieure de l'ectoderme corfuillier.

Chez les Styliolacées, qui ont une vie larvaire prolongée, la coquille atteint, avant la métamorphose, le double de la longueur de la coquille larvaire (pl. VI, fig. 6 et 7 ). La larve se détache de sa coquille à mesure que cette dernière est formée et n'y reste attachée que par le point d'insertion du muscle columellaire (pl. VII, fig. 9 et 10). Néanmoins la coquille larvaire persiste pendant toute la vie de l'animal.

Il en est de même chez Creseis, où la coquille larvaire a la forme de l'olive d'une sonde boutonnée. L'animal garde cette partie inférieure de sa coquille pendant toute sa vie; elle reste tapissée par l'ectoderme et s'épaissit visiblement avec l'âge.

Parmi les Thécosomes campyloconques, la cơpuille larvaire persiste chez les Cymbuliacées assez longtemps, mais se distingue à 
peine de la cocquille du jeune. Ces deux portions de la coquille du jeune tomberont ensemble lors de la métamorphose. La partie larvaire persiste toute la rie chez les Limacinées et n'est séparée par aucune limite de la partie de la coquille qui se forme ultérieurement. Cette coquille des Campyloconques est ronde à son sommet et s'enroule tantôt dans un plan, tantôt en biais.

Chez les Gymnosomes enfin, la coquille larvaire se distingue nettement de la partie qui vient s'y ajonter ensuite. Cette dernière est très-variable de forme, d'après les olsservations de Krohn, et tombe lors de la métamorphose. Cette corguille larvaire est sphérique on ovoïle; la partic qui vient s'y ajouter'est ouverte en forme de cornet. Elle ne reste, de même que chez Creseis, en contact avee l'ectoderme coquillier que juste le temps nécessaire à sa sécrétion.

Les jeunes, chez les Orthoconques, nagent déjà tont à fait à la manière des adultes, c'est-à-dire comme des papillons, d'où leur vient le nom rulgaire de farfalle di mare. Ils battent l'eau de leurs nageoires, la tête en haut, la coquille en bas, obliquant souvent à droite on à gauche, mais ne se renversant jamais la tête en bas, pas du moins de leur propre gré.

Les jeunes Gymnosomes se munissent, comme on le sail, après la métamorphose, de trois couronnes ciliaires et progressent dans l'eau à la maniòre des larves de vers. J'ai observé beaucoup de formes différentes de ces larves; mais, comme je ne sais pas encore à quelles espèces les rapporter, je préfère passer sous silence ces observations, qui n'auront que peu d'intérêt tant qu'elles ne seront pas complétées.

Le demier système d'organes qui apparaisse chez le jeune est naturellement celui des organes de la génération. Je n’ai recueilli que quelques notes sur ce sujet, se rapportant aux genres Creseis et Hyalocylis. Chez Crescis, on voit une conche de cellules (pl. VI, fig. 4, Gh) se diflérencier à la surface du sae nutritif $(\sigma)$, à ganche, entre ce sac et la paroi qui sépare le corps de la cavité palléale $(m p)$. Chez des individus plus âgés (pl. VI, fig. 3, Gh), celte couche de cellules s'étend tout le tour du sac nutritif, qui est en train de tomber en dégénérescence. Du côté opposé à celni où cette couche est en contact avec la paroi dle la carité palléale, la gylande présente un grand développement et se lobe à la surface. Une question importante à résoudre est celle de saroir si cette couche, qui deviendra la glande génitale hermaphrodite, provient du sac nutritif ou de la paroi de la cavité palléale; en d'autres termes, si elle provient de l'entoderme 
ou de l'ectoderme. Je me prononce pour la première alternative, parce que le développement de la glande se fait de la même manière chez Hyalocylis, autour des restes du sae nutritif, qui est ici plus éloigné de la cavité palléale. Une certitude absolue ne pourrait s'obtenir qu'en suivant le développement de cet organe pas à pas, chose bien difficile à faire avec des animaux dont la pêche ne vous procure que de loin en loin des individus de l'âge qu'on désire. Néanmoins, je crois m'être assuré que la glande hermaphrodite résulte d'une seission de la paroi du sac nutritif.

En tous cas, Ies œufs et les spermatozoaires m'ont paru se former aux dépens de cette couche cellulaire, unique dans l'origine. Si l'on cherchait à faire dériver les deux produits sexuels des deux feuillets embryonnaires, on pourrait supposer que les æufs, cui prennent naissance à l'extérieur, proviennent de l'ectoderme, le sperme de l'entoderme. Mais, je le répète, dans mon opinion, la glande entière et ses produits proviennent de l'entoderme.

Je n'ai pas d'observations sur le développement des organes sexuels efférents.

Chez les Thécosomes, les produits sexuels n’atteignent leur maturité complète que chez des individus qui ont pris toute leur croissanee. Je n'ai jamais pu obtenir de pontes d'exemplaires moins développés. Chez les Gymnosomes, Krohn a vu des individus jeunes de Pneumodermon avoir déjà du sperme à l'état de maturité. Cela n'est pas très-étonnant. puisque nous sarons, depuis les recherches d'Eisig, que le sperme se forme de très-bonne heure chez le Lymnée, où l'on trouve des spermatozaires tout formés dans la glande hermaphrodite d'individus encore tout jeunes et chez lesquels cette glande ne peut encore déverser son contenu à l'extérieur. Les cufs mùrissent beaucoup plus tard que les produits sexuels mâles.

Le cas que j’ai observé d'un Gymnosome ressemblant beauconp à des jeunes du genre Clio, et dont j'ai obtenu plusieurs pontes, n'en est pas moins remarquable, malgré les observations' de Krohn. J'ai pris plusieurs exemplaires de cet animal, tous de même taille et parfaitement pareils entre eux. Tous présentaient un caractère qui appartient à la fin de la seconde période larvaire des Gymnosomes, à savoir: la présence de deux couronnes de cils, l'une au milieu du corps, l'autre à son extrémité postérieure. Et cependant j’ai gardé ces animaux en captivité et en parfait état de santé pendant plus de trois semaines, sans qu'ils perdissent ces couronnes de cils ou dérelop- 
passent une branchie. C'est pendant cet espace de temps qu'ils m'ont fait quatre à cinq pontes d'œufs qui se sont parfaitement développés. Tout ceci me donne à penser qu'il s'agil ici d'un genre nouveau et non pas d'un exemple de Ptérop̀ode capable de pondre des œufs avant d'avoir atteint l'état adulte. Néanmoins, comme je n'en suis pas parfaitement sûr, je préfère ne pas risquer de créer encore un synonyme inutile; je place provisoirement ce Gymnosome comme espèce nouvelle dans le genre Clio, aux jeunes duquel il ressemble. On trouvera plus loin une description détailléc de cet animal. 


\section{DES DIVERS TYPES DE DÉVELOPPEMENT DES PTÉROPODES}

\section{COMPARÉS ENTRE EUX}

\section{ET AVEG GEUX DES AUTRES MOLLUSQUES.}

En traitant du développement des Ptéropodes, j’ai déjà indiqué les différences qu'ils présentent aux diverses périodes de leur évolution; mais ces données sont éparses et décousues. Je vais donc les réunir et examiner si elles ne suffisent pas pour fonder une classification naturelle du groupe.

De Blainville a divisé lout l'ordre en deux familles, les Thécosomes et les Gymnosomes. Cette coupe me paraît parfaitement naturelle. Il y aurait, il est vrai, beaucoup à redire aux noms qu'a choisis ce savant distingué. En effet, à l'état larvaire tous les Ptéropodes sont thécosomes, tous sont munis d'une coquille externe, produit de la sécrétion de l'ectoderme. Si, au contraire, ces désignations ne s'appliquent qu'aux animaux à l'état adulte, nous remarquons que les Cymbuliacées, qui doivent probablement rentrer dans les Thécosomes, sont en réalité dépourvues de coquille. La coquille étant une sécrétion de l'ectoderme, soit à la surface, soit dans l'intérieur d'une invagination dans les cas où celle-ci persiste, il est clair que ce que l'on a jusqu'à présent désigné comme la coquille interne des Cymbuliacées ne répond en aucune façon aux coquilles internes ou externes des autres Mollusques. C'est un simple durcissement de la eouche dermique; c'est une pièce de squelette si l'on veut, ce n'est pas une coquille. Ainsi done, si les Gymnosomes ne sont nus qu'après la fin de leur période larvaire, il est aussi parmi les Thécosomes une tribu qui perd sa coquille lors de la métamorphose et reste ensuite en réalité toute nue. Mais, ces réserves une fois faites au point de vue morphologique, il faut reconnaître que la classification proposée par de Blainville est naturelle, que les termes qu'il a choisis sont sinon tout à fait justes, du moins fort commodes. Je l'adopterai telle quelle, tout en réservant la question de savoir si les Cymbuliacées n’ont pas plus d'alfinité pour les Gymnosomes que pour les Thécosomes.

Les essais qui ont été fails de ponsser la classification plus loin 
se bornent généralement à une division des Thécosomes en trois tribus, celles des Cymbuliacées, des Hyaléacées et des Limacinées, auxquelles on a ajouté une ou deux tribus entièrement fossiles. Ces groupes me paraissent justes, mais il me semble qu'ils ont entre eux des relations de parenté dont on n'a pas tenu compte. Les genres, enfin, qui composent ces tribus, sont, du moins pour les Hyaléacées, un chaos qui serait inextricable, si nous ne possédions les magnifiques dessins qu’ont donnés Eydoux et Souleyet. Les formes des larves peuvent seules nous donner un moyen sûr d'établir des coupes génériques naturelles chez des êtres aussi polymorphes dans leur état adulte.

J'ai fait roir que le premier rudiment de la coquille peut être: $1^{\circ}$ ovoïde, $2^{\circ}$ pointu, ou $3^{\circ}$ aplati en forme de verre de montre. Dans le premier cas la coquille larvaire peut avoir la forme d'un cornet arrondi au sommet (Hyaléacées); elle est alors le plus souvent striée transversalement et parfois élargie latéralement (Cleodor $($ ). Elle peut persister pendant toute la vie de l'animal et continuer à former le sommet de la coquille (Hyalcea); ou bien elle peut être évacuée par l'animal, qui referme sa coquille au-dessus par une cloison transtersale, et se casser (Cavolinia, Hyalocylis), ou bien le sommet de la coquille larvaire a la forme d'une olive de sonde (Creseis). Elle est alors lisse et persistante.

Dans le deuxième cas, la coquille larvaire étant pointue, elle est toujours lisse et persistante (Cleodora, Styliola).

Enfin, dans le troisième cas, le rudiment aplati de coquille forme le sommet d'une coquille larvaire nautilö̈de (Limacinées, Cymbuliacées), ou simplement ovoïde (Gymnosomes). Lorsque la coquille est enroulée, elle peut être persistante (Limacinées) ou caduque (Cymbuliacées). Lorsqu'elle est persistante, elle peut s'enrouler en volute (Spirialis) ou en spirale (Limacina). Lorsqu'elle est caduque, elle est enroulée dans un plan et forme un tour et demi avant de tomber; le dernier demi-tour se détache du tortillon et peut être rapidement élargi (Cymbulia) on peu ouvert (Tiedemannia).

Le pied peut se séparer peu après sa formation en trois lobes, dont un médian devient le pied proprement dit et les deux latéraux deviennent les nageoires (Hyaléacées); cette division en lobes peut se montrer un peu plus tard (Creseis), ou même très-tard (Styliolacées). Le pied peut aussi être arrondi, échancré au milieu, muni d'un opercule à sa face inférieure et d'un appendice filiforme qui prend naissance 
au milieu de son bord libre, au-dessus de l'écrancrure médiane (Cymbuliacées). Il se divise aussi dans ce cas en trois lobes qui forment les nageoires et le pied proprement dit, mais ces lobes ne sont jamais aussi distincts que chez les Hyaléacées.

Le pied peut être aussi très-petit, en forme de goultière (Gymnosomes). Dans ce cas son extrémité s'étire en une sorte de lanière ciliée qui tombe plus tard. Il donne naissance à um pied en forme de fer à cheval et probablement aussi aux nageoires. 11 ne porte pas d'opercule.

Le roile peut rester petit et tomber de bonne heure (Hyaléacées), ou bien il peut persister très-longtemps et atteindre de grandes dimensions (Cymbuliacées, Gymmosomes, Styliolacées). Il peut s'échancrer à son bord externe, si bien que chaque disque se sépare en deux lobes (Créséidées, Cymbuliacées).

- L'anus peut se trouver originairement sur la ligne médiane ventrale, et se déplacer rers la gauche à mesure que la cavité palléale, qui prend son origine à droite, gagne en extension (Orthoconques); ou bien l'anus peut se trouver dès l'abord du côté droit, où il reste, la cavité palléale prenant naissance au côté dorsal (Campyloconques et Gymnosomes). La masse nutritive s'arrange en deux sacs, dont l'un peut prédominer énormément sur l'autre (Orthoconques), ou qui sont égaux entre eux et s'ourrent à la même hauteur dans l'estomac (Campyloconques et Gymṇosomes).

Il peut se former dès l'abord des dents triturantes dans l'estomac (Hyaléacées), ou bien leur apparition peut être précédée par une plaque dentaire unique (Styliolacées et Campyloconques).

Le muscle columellaire peut être unique, ou bien il peut se trourer" un second muscle longitudinal plus rapproché de la face ventrale (quelques Gymnosomes, sinon tous).

Enfin, la durée de la vie larvaire peut être très-courte (Hyaléacées) ou très-prolongée, et dans ce dernier cas, qui cst celui de la grande majorité des Thécosomes, les organes larvaires prennent un grand développement et les organes définitifs ne se forment que très-tard. La période larvaire proprement dite peut être assez courte, mais suivie d'une seconde période larvaire toute dilférente (Gymnosomes).

En combinant les caractères qui viemment d'ètre énumérés avec les caractères que présentent les animaux à l'ćtat adulte, j’arrive au tableau suivant, que je ne donne pas comme une classification complète et définitive, mais seulement comme plus approfondie que tous 
les systèmes proposés jusqu'à ce jour. Je ne parlerai que des genres que j'ai eu l'oceasion d'observer par moi-même.

Ordre des Ptéroporles (Cuv.). - Céphalophores hermaphrodites, à pied trilobé, les lobes latéraux étant développés en deux grandes nageoires, munis à l'état larvaire d'une coquille externe véritable et d'un voile.

Premier sous-ordre. - Thécosomes (Blv.). - Ptéropodes dont le corps est protégé à l'état adulte soit par une coquille calcaire, soit par une pièce eartilagineuse, formée dans la couche dermique. Une seule période larvaire. Cavité palléale s'ouvrant à droite de l'anus et munie d'un bouclier vibratile.

Première famille. - Orthoconques. - Thécosomes à coquille larvaire droite ou peu recourbée, à conearité dorsale. Carité palléale s'ouvrant du côté droit. Anus sur la ligne médiane ventrale ou du côté gauche. Pas d'opercule. Nageoires verticales. Une véritable coquille externe.

Première tribu. - Hyaléacées (Rang). - Orthoconques à coquille larvaire en forme de cornet ou de dé à coudre, arrondie au sommet. Période larvaire abrégée et métamorphose hâtive. Cavité palléale munie de branchies.

Premier genre. - Cavolinia (Giöni). - Coquille larvaire en forme de cornet arrondi au sommet, aplatie dans le sens dorso-ventral et élargie dans le sens latéral; courbée en quart de cercle, la convexité de la courbure étant rentrale; striée transversalement de lignes très-nettes et régulières. Coquille de l'adulte fermée au sommet par une cloison transversale qui la sépare de la coquille larvaire, laquelle est abandonnée par l'animal el se casse; subglobuleuse, tricuspidée postérieurement, la pointe du milieu répondant à la coquille du jeune. Ouverture de la coquille étroite, en forme de fente à bord dorsal prolongé, à bord ventral recourbé en dedans, prolongée sur les côtés jusqu'aux pointes latérales. Manteau prolongé en quatre appendices en forme de lanières. (Cavolinia tridentata $=$ Hyalca trident (Lam.), Cavolinia gibbosa $=$ Hyalca gibbosa.)

Deuxième genre.-Hyalcea (Lamk.).- Coquille larvaire en forme de cornet arrondi en bas, régulièrement striée de lignes transversales, courbée en quirt de cercle, la convexité de la courbure ćtant ventrale, persistant jusque ehez l'adulte. Coquille de l'adulte terminée en bas par la coquille larvaire, sans eloison transversale; fendue en avant, la fente descendant très-bas sur les côtés; de forme variable, générale- 
ment plus mince que chez le genre précédent, marquée de stries d'accroissement. Manteau formant de deux à quatre prolongements en forme de lanières qui sortent par les angles postérieurs de la fente.

Troisième genre. - Hyalocylis, g. n. - Coquille larvaire en forme de dé à coudre, sans stries, presque droite, caduque. Coquille de l'adulte cannèlée transversalement, terminée en arrière par une cloison transversale qu'entourent les bords fracturés de la coquille larvaire; légèrement courbée, la courbure à convexité ventrale; circulaire sur une coupe transversale. Point d'appendices au manteau. (Hyalocylis striata $=$ Cleodora striata (Rang.).)

Deuxième tribu. - Styliolacées. - Orthoconques à coquille larvaire en forme de cornet, pointue en bas, lisse, persistant pendant toute la vie. La couche ectodermique se relire de chaque portion de la coquille aussitôt après l'avoir sécrétée; le point d’insertion du muscle columellaire reste près de la pointe de la coquille larraire. Pas de cloison transversale, pas d'épaississement de la coquille. Période larvaire prolongée, disques moteurs acquérant un grand déreloppement, sans échancrures au bord externe.

Premier genre. - Cleodora (Per.). - La métamorphose a lieu avant que la coquille définitire ait aiteint la même longueur que la coquille larvaire; roile moyen. Coquille de l'adulte aplatie d'avant en arrière, étirée en pointes sur les côtés, terminéc en bas par la coquille larvaire, mince, hyaline, montrant souvent des stries d'accroissement peu nettes.

Deuxième genre. - Styliola (Les. diagn. emend.). - Métamorphose tardive, roile très-développé. Coquille de l'adulte formant un cône .très-allongé, la pointe formée par ln coquille larvaire; à section circulaire, munie d'une arète dorsale. Cavité palléale dépourvue de branchies. (Styliola subulata $=$ Cleodora subulata (Q. et G.).)

Troisième tribu. - Créséidées. - Orthoconques à coquille larraire ovale se continuant en un cône; sans stries d'accroissement. Coquille de l'adulte en cône très-allongé, presque cylindrique, terminée en bas par un renflement, comme l'olive d'une sonde boutonnée, qui n'est que la coquille larvaire. Celle ci est épaissie et d'un ton rougebrun. Point de cloison transrersale. Métamorphose tardire; voile très-développé, chaque moitié étant séparée en deux lobes par une échancrure latérale. Sommet du lobe médian du pied allongé et couvert de cils. Nombreuses petiles cellules vibratiles disséminées dans la cavité palléale; point de branchies. 
Genre C'reseis (Rang.). - Avec les caractères de la tribu. (Creseis aciculat $=$ Cleodora acic. (Rang.) $=$ Styliola acic. (Bronn.)

Deuxième famille. - Campyloconques. - Thécosomes à période larvaire prolongée. Premier rudiment de eoquille en forme de verıe de montre ; coquille larvaire enroulée, à convexité dorsale. Pied trilobé, le lobe médian muni d'un opercule.

Premièretribu. - Limacinées (Gray). - Campyloconques à coquille larvaire persistante, formant le sommet du tortillon. Enroulemen ten volute à développement plan (Spirialis) ou en spirale à développement conique (Linacina).

Deuxième tribu. - Cymbuliacées (Gray).-Campyloconques à coquille larvaire cadurue formant un tour et demi de spire, le dernier tour se détachant dı tortillon. Animal protégé plus tard par une pièce cartilagineuse recouverte par l'ectoderme, résultant du durcissement de la couche dermique et non d'une sécrétion de l'ectoderme dans une cavité d'invagination. Appendice filiforme au milieu du bord libre du pied. Lobe médian et nageoires peu différenciés, les nageoires s'étalant sur les côtés du corps.

Premier genre. - Cymbulia (Pel'.). - Le dernier tour de la coquille larvaire à angle très-ouvert. Pied de l'adulte séparé en trois lobes par deux échancrures profondes.

Deuxiëme genre. - Tiedemannia (Delle Ch.). - Dernier tour de la coquille peu agrandi. Pied de l'adulte ì bord presque entier.

Deuxième sous-ordre.—Gymnosomes(Blv.). - Coquille larvaire ovoïde, continuée elı un cône ouverí, à ammeaux ou à stries transversales; caduque. Période larvaire prolongée, suivie, après la métamorphose, d'une seconde période larvaire caractérisée par la présence de trois couromnes vibratiles caduques (chez tous?), dont aucune ne répond au roile. Extrémité du pied allongée en une longue lanière ciliée pendant la première période larvaire. Nageoires latérales, complétement séparées du pied chez ladulte.

Je n'entrerai pas dans la description des genres de la famille des Gymnosomes, n'ayant pas eu l'occasion d'observer un nombre suffisant de leurs représentants. Je me bornerai à décrire la forme nouvelle dont j’ai observé et rapporté le développement.

Clio armantiuca, n. sp. (pl. X, fig. 10). - Animal en somme fusilorme ou oroïde suivant son état de contraction ou de dilatation. Epiderme tout entier couvert d'un duvet de cils extrêmement fins $(c)$. Deux couronnes de cils moteurs, l'vue un peu en arrière du milieu 
du corps, l'autre près de l'extrémité postérieure $(\mathrm{cm})$, chaque couronne étant composée d'une rangée de cils portés sur un seul rang de grandes cellules. Quatre petits mamelons de l'épiderme converts chacun d'une houppe de cils, à la base de la tête, deux sur les côtés et deux à la face dor'sale $\left(c^{\prime}\right)$. Epiderme muni d’une quantité de glandes unicellulaires à aspect graisseux, faisant saillie à sa face interne $(G c)$; un certain nombre de glandes cutanées pluricellulaires vers le milieu du corps $\left(G c^{\prime}\right)$. Fibres musenlaires cutanées, longitudinales et circulaires (ß.). Quatre tentacules très-rétractiles placés en carré autour de la tête, deux du côté ventral et deux du côté dorsal $(t)$. La surface de ces tentacules est munie de rangées transversales de cellules saillantes cylindriques, surmontées chacune d'une soie raide et droite $\left(c{ }^{\circ}\right)$. Entrée de la bouche présentant sur les côtés deux excavations qui forment, en se retournant, deux bourrelets courerts de petites verrues dont chacune porte une soie raide $\langle b s\rangle$. Radule composée d'une rangéc médiane re petites dents en forme de houes et de deux langées latérales de dents longues et acérées en forme de pioches $(\omega r)$. Pied composé d'une partie inférieure horizontale en forme de gouttière pointue aı bout et de deux parties latérales qui s'élèvent verticalement jusqu'à la base de la tête. Bord supérieur des nageoires en contact à la base avec ces parties latérales du pied. Nageoires $(p n)$ pointues à l'extrémité latérale. Le bord inférieur, presque droit, est garni d'une rangée de cirrhes ou mieux de petites palettes raides, mobiles à leur bàse et battant l'eau tout d'une pièce de temps à autre $(c r p)$. Muscles ( $/ p)$ disposés en plusieur's couches et se rencontrant à angles aigus, ainsi que cela a été décrit par Gegenbaur et Krohn pour les autres Gymnosomes. Olocystes pleins d'une quantité innombrable de petits otolithes fusiformes. Glande génitale trèsdéveloppée et occupant toute la partie postérieure du corps, montrant les ovules à l'extérieur $(G o)$ et les petites grappes de cellules mères du sperme à l'intérieur $(G t)$.

Cet animal nage dans l'eau tant à l'aide de ses nageoires qu'à l'aide de ses cils moteurs. Sa longueur comporte de 2 à 2 millimètres et demi. Il est excessivement rétractile et change de forme à chaque instant. Ni la compression ni aucun des stratagèmes que j’ai inventés n'ont pu l'obliger à rester un instant sans tourner et se tordre de toute manière. Il possède, en outre, la faculté da rentrer non-seulement ses tentacules, mais encore sa tête, son pied el ses nageoires, faculté dont il abuse au point d'énerver l'observateur le plus persévérant. Ce n'est 
donc que sous toutes réserres que je donne la description des tentacules et des organes buccaux, que je n'ai réussi à voir que trois ou quatre fois, et cela seulement pendant un instant. Je crois cependant pouvoir aftirmer que les tentacules et les sacs buccaux portent des soies droites et non des rochets.

Après avoir comparé le développement des divers Ptéropodes, je passe à la comparaison cmbryogénique de cet ordre avec les autres Mollusques et avec des animanx qui appartiennent ou qui ont été, jusqu’à présent, classés dans d’autres embranchements.

Les procédés de division des premières cellules embryonnaires ou sphères de segmentation, tels que je les ai décrits pour les Ptéropodes, sont très-répandus dans le règne animal. J'ai fait voir que la disparition du nucléus après la fécondation, puis avant chaque segmentation, et son remplacement par deux centres d'altraction, s'observe chez des Annélides (Alciope), des Tuniciers (Doliolum), des Cœlentérés (Geryonia el $C y d i p p e)$. Depuis lors, des phénomènes analogues ont été observés par Flemming chez les Anodontes. Les observations de Warneck permettent de croire que les choses ne se passent pas autrement chez les Gastéropodes pulmonés, et moi-même j’ai retrouvé ces mêmes étoiles moléculaires présidant à la segmentation chez. les Hétéropodes et les Gymnobranches.

Je n'ai encore jamais observé de segmentation précédée d'une division proprement dite du nucléus, mais je ne me hasarderais pas à affirmer que c'e mode de fractionnement n’existe pas dans le règne animal. 11 semble, au contraire, bien avéré qu’il se rencontre chez certains vers. Mais, même dans le cas où le nueléus disparaît, sa substance n'en forme pas moins, selon toute vraisemblance, la partie centrale des étoiles moléculaires; ct comme c'est dans le centre de ces étoiles que réapparaissent les nouveaux nucléi, il est permis de supposer que ceux-ci sont, du moins en partie, composés de la substance même du nucléus avant la division.

J'ai dit que l'embryon des Ptéropodes est composé de cellules formatives et de cellules nutritives, mais j'ai fait voir en même temps quel sens il fallait attacher à cette dénomination de partie nutritive du vitellus et de cellules nutritives. Plusieurs observateurs se sont trompés en donnant à cette distinction un sens trop absolu et en attribuant aux cellules formatives seules le rôle de créer les tissus de l'embryon, landis que les cellules nutritives se comporteraient d'une 
manière passive et seraient simplement absorbées petit à petit par les premières. J'ai montré que les cellules nutritives, chez les Ptéropodes, sont au contraire un atelier de fabrication où s'élabore constamment de la substance protoplasmique qui vient former la majeure partie des tissus embryounaires.

Chez les Gymubranches et les Pulmonés, où les cellules nutritives sont bien moins grosses et renferment une provision de nourriture bien moins considérable, les auteur's s'accordent à faire dériver directement de ces cellules les éléments histologiques du feuillet gastrique. Sous ee rapport, les Ptéropodes établissent une transilion trèsimportante entre le déreloppement des Gymmobranches et celui des Céphalopodes, entre l'holoblastie et la méroblastic. Supposons, en effet, que la quantité de matière nutritive contenue daus le vitellus de Cavolinia vienne à augmenter à tel point que la petite quantité de protoplasme perde son empire sur celte masse inerte trop abondante; nous verrons alors le feuillet externe lout entier se former par des divisions du protoplasme à la surlitee de la masse nutritive compacte el unique, et cela en commençant par le pôle aboral. Puis cet ectoderme primitif se dédoublera, de même que chez les Ptéropodes, en un ectoderme et un mésoderme. Or e’est là précisément ce qui s’observe chez les Céphalopodes, d'après les travaux le Kölikiker et d'Ussow.

J'ai décit avec soin l’ordre et le mode de formation des sphères de segmentation chez les Ptéropodes, parce que les mèmes lois de distribution des premières cellules se retrouvent chez une foule d'autres Céphalophores. Warneck les a très-hien observées et décrites chez Limax et Lymnceus ; j'aurai bientòt occasion de les décrire pour les Hétéropodes, les Gymnobranches et mème pour quelques Lamellibranches. L'on peut, dès à présent, considérer ce mode de segmentation comme typique pour les Céphalophores et, jusqu’à un certain point, pour les Lamellibranches el même les Rotifères. Chez les Céphalopodes, cette loi ne serait, d'après ce qu'en dit Ussow, pas aussi différente qu'on l'a cru de celle des Gastéropodes.

Le résultat du fractionnement est, daus tous les cas où le vitellus de nutrition n'est pas abondant, la formation d'une sphère cellulaire, plus ou moins creuse, dont l'un des côlés est occupé par des cellules plus petites, plus riches eu protoplasme, l'autre côté, par des cellules plus grosses et plus abondamment pourvues de matière nutritive. Le premier de ces cleux côtés répond à celui par lequel sont sortis les corpuscules excrétés. Le second côté, le côté nutritif, s'aplatit, puis 
rentre dans l'autre, et il en résulte une forme que je ne puis mieux comparer qu’à celle que prend une balle de caoutchouc percée lorsqu'on la presse entre les doigts, Cette invagination du côté nutritif a été observée par moi chez Tritonia, Elysia (Actcon), Phyllirhoë, Limax et chez les Héléropodes; à en juger par les figures de Davaine et mène de Carus, elle se retrouverait chez divers Lamellibranches. Lereboullet l'a observée chez Lymmitus : seulement ces divers auleurs représentent l'invagination sams en comprendre la portée, ou tout au moins sans indiquer quelle est la moilié de l'ébauche embryonnaire qui se renfonce dans l'autre. Lereboullel seul a suivi pas à pas le sort ultérieur le cette cavité d'enfoncement, et s'est assurć (fu’elle donnait naissance au tube digestif, que son ouverture n'était autre que celle de la bouche. Celte observation concorde entièrement avec les miennes sur les Hétéropodes, les Ptéropodes et Limax. Chez ce dernier genre surtout, j’ai vu de la manière la plus évidente le canal d'invagination derenir la bouche ou, pour mieux dire, le rardia. Il en est de mème chez. Doris d'après les observations de Langerhans. L'ouverture d'invagination paraît se refermer chez les Gymuobranches et les Ptéropodes, mais la bouche se forme bientôt à l'endroit même où le feuillet externe vient de se rejoindre.

Chez les Céphalopodes, la bouche se forme, non pas au pôle nutritif même, mais dans son voisinage, du côté dorsal. La distance qui la sépare du pôle est d'autant moindre que la hernie du vitellus de nutrition est moins considérable; très-grande chez Sepia, elle est presque nulle chez le Céphalopode observé par Grenacher.

L'on pent done raisonmablement espérer que l'homologie complète de la bouche dans tous ces cas ne tardera pas à être démontrée d'une manicre parfaitement satisfaisante.

Ouclques Prosobranches sembleraienl, d'après les données peu nombreuses que nous possédons, se développer sur un type bien différent. D’après de Tacaze-Duthiers et d'après Salensky, la bouche se creuserail au pôle formatif chez. Vermetus et chez Calyptraxa. Chez Purpura, à en juger par le travail de Selenka, la bouche se trouverait bien au pôle nutritif; mais, sous un autre rapport, ce type, tel qu'il nous est décrit, ne peut absolument pas se ramener à ce que nous sarons du développement des autres Céphalophores: l'une des sphères de segmentation se comporterait d'une manière complétement passive et le fenillet gastrique se formerait par un retroussement du feuillet externe autour de cette sphère! Il serait inutile, pour le moment, el 
avec des matériaux aussi bruts, de chercher à étabïr ici des homologies et à atteindre déjà le but que doit se proposer toute personne qui travaille à l'avancement de l'anatomie et de l'embryogénie com. parées.

Le mode de formation du tube digestif est très-simple dans les cas d'invagination ; la couche de cellules nutritives invaginée donne naissance à l'estomac, à l'intestin el aux sacs mutritifs, par une simple différenciation de ses éléments histnlngiques. La partie médiane et ventrale de la paroi de cette cavité prend le caractère d'un épithèle simple, qui se courre de cils et derient le tube digestif, tandis que les parties latérales, renflées en forme de poches, ont des parois composées de grosses cellules nutritives et deriennent les deux sacs nutritifs. Tous ces éléments histologiques descendent directement des cellules nutritives qui entouraient la cavité d’invagination primitive.

Chez les Ptéropodes, surtout chez les Orthoconques, cette formation paraît moins simple et moins claire au premier abord; mais $\mathrm{mm}$ examen attentif permet de la ramener au tỵpe précédent, de même que le déreloppement du feuillet gastrique peut se ramener au déreloppement par invagination. Les procédés sont comparables, seulement ils sont voilés et modifiés jusqu'à un certain point par l'abondance de la substance nutritive.

Chez Acera et chez Aplysia, d'après Ray Lankester et Langerhans, le tube digestif tout entier l'ésulterait de la différenciation de cellules ectodermiques, qui pousseraient de l'extérieur en denx points répondant à la bouche et à l'anus. Ces deux cordons cellulaires se rencontreraient au centre de l'embryon, où ils formeraient l'estomac. Les cellules nutritives se comporteraient chez Aplysia d'une manière tout à fait passive.

Chez les Rotifères, Salensky a observé aussi la formation du tube digestif par une simple différenciation des cellules, riches en substance nutritive, de l'entoderme. La partie médiane de cette carité primitive répond à l'estomac el à l'intestin; les parties latérales ne forment pas deux, mais bien quatre saes, soit deux paires, dont la paire antérieure donnera naissance à des glandes en relation avec les fonetions digestives, la paire postérieur'e aux glandes sexuelles.

Chez les Céphalopodes, il paraitrait que la partie centrale et inférieure du tube intestinal se forme par une invagination de l'ectoderme partant de l'anus. Ce nıde de formation, que mes propres obserrations ne confirment pils, lliffère foncièrement de ce que nous 
savons des Céphalophores. En tous eas, l'homologie embryogénique du tuJje intestinal entre les Ptéropodes, les Hétéropodes, plusieurs Gymnobranches et les Pulmonés me paraît mise hors de doute.

Pour les Céphalophores, comme pour les Céphalopodes, les Lamellibranches et les Rotilères, l'usophage el la bouche résultent d'une invigination de l'ectorlerme, ou d'un simple enfoncement, dans les cas où l'ouverture d'invagination primitive persiste. Cette ouverture répond done non pas à la bouche, mais au cardia. Toute la portion du tube intestinal située au-dessous du cardia dérive de l'entoderme, toute lu portion située au-dessus du cardia provient d'un enfoncement secondaire de lectoderme. Celte dernière partie donne naissance à l'œsophage ef à ses parties annexes, les mandibules, les sacs à crochets, le sac de la radule; les homologies de ces parties des Céphalophores avec celles des Céphalopodes sont assez compliquées; chez les Rotifères, l'appareil de mastication paraît comparable à la radule des Céphalophores, à en juger par la description de Salensky .

Les parties latérales de l'entoderme ne donnent pas directement naissance au foie. J'ai démontré ce point en ce qui concerne les Pléropodes, je le démontrerai bientôt pour d'autres Céphalophores. Ces parties latérales forment deux sacs qui se fusionnent en un seul; ce dernier joue peut-être pendant un certain temps le rôle de foie. Le foie véritable résulte d'évaginations de la paroi du tube digestif. Les observations de Lereboullet pour Lymnæus donnent à penser qu’il pourrait bien en être de même ici, et que les diverticules que cet auteur a pris pour un double canal cholédoque en voie de formation pourraient n'être que le foie naissant. Chez les Céphalopodes, Ussow nous apprend que le foie naît aussi comme diverticule du tube intestinal, diverticule qui n'a aucune relation avec le vitellus de nutrition. Chez Brachionus enfin, Salensky a montré que les glandes qui débouchent sur les côtés à la partie supérieure de l'estomac résultenl d'une transformation direcle des diverticules supérieurs de l'entoderme de l'embryon. Il s'ensuit que ces glandes des Rotifères sont comparables aux sacs nutritifs des larves de Céphalophores.

La glande sexuelle des Ptéropodes se forme aux dépens du sac nutritif, et par conséquent aux dépens de l'entoderme. Salensky a montré que chez Brachionus l'ovaire se forme aussi aux dépens de la partie latérale inféricure du feuillet gastrique; chez le mâle de cetle espèce, le feuillet gastrique lout entier devient un vaste testicule et le tube intestinal ne se développe pas. 
L'invagination du pôle aboral a été nommée indifféremment coquitlière ou préconchylienne. Le second de ces termes n'est applicable qu'aux Ptéropodes et à d'autres Mollusques marins à coquille externe. Le premier est plus juste à un point de vue général et surtout si on l'applique aux Mollusques à coquille interne. Cet enfoncement de l'ectoderme est silué chez les Ptéropodes orthoconques, où sa position peut ètre déterminće avec exactitude, un peu du còté ventral du pôle formatif. Il en est de mème chez Limax, Phyllirhoë et les Hétéropodes, et il en est aussi de mème chez Lymncous et chez Ostrcea, à en juger par la description de Ganine, par les dessins de Lereboullet et de Salensky, et par mes propres dessins sur ce dernier genre. Chez les Céphalopodes, sa position m’a paru un peu différente; elle m’a paru coïncider avec le pôle formatif mème. Cette différence n’est pas bien grande, si tant est qu'elle existe.

Le rôle physiologique de cette invagination varie du tout au tout suivant que la coquille est interne ou externe. Dans le premier cas, qui est celui des Céphalopodes à coquille interne et des Limaces, son utilité est évidente. L'enfoncement s'approfondit, son ouverture se referme entièrement et la coquille se sécrète dans la carité ainsi formée. Dans le second cas, l'invagination se ‘ retourne et la coquille se forme sur la saillie qui en résulte. Puis, le sommet de la coquille une fois formé, la saillie épaisse s'étire et devient le point de départ d'un bourrelet, qui sécrète le bord de la coquille, présidant à sa croissance, à savoir: le bourrelet du bord du manteau. L'utilité physiologique de ce dernier procédé mérite d'être examinée, car si cette utilité pouvait être démontrée, ce trait d'organisation aurait pu être acquis par les larres en vertu de la loi de l'adaptation aup circonstances ambiantes. Si, au contraire, l'utilité en est nulle, cet organe rentrera forcément dans la catégorie des organes dits rudimentaires, de ceux qui ne doivent leur existence actuelle quà la loi de l'hérédité.

Chez les Ptéropodes orthoconques il pourrait sembler que la formation d'une cavité d'invagination ait pour but d'obtenir subitement et à un moment donné une saillie de la forme voulue pour servir de moule au sommet de la coquille. Et pourtant le même but pourrait être atteint par des moyens bien plus simples. Mais chez les Ptéropodes campyloconques, chez les Hétéropodes et les Nudibranches, le sommet de la coquille a la même forme que l'extrémité postérieure de l'embryon. Nous ne voyons, dès lors, pas à quoi celte invagination pourrait servir. 
Mais, s’il n'atteint pas un but déterminé, cet enfoncement serait-il peut-être produit, en yuclque sorte fortuitement, par le fait d'une croissance trop rapide des tissus, au point central où commence la formation du bourrelet du manteatu? Cette opinion pourrait se soutenir. Le fait que l'invagination n'est pas toujours très-marquée, qu'elle peut être remplacée par un simple épaississement légèrement excavé, ainsi que cela a été observé par Semper chez l’Ampullaria polita, par Salensky et par moi chez l'Huître, on par une excavation un peu plus prolonde, comme l'a vue Salensky chez Calyptrcea, montre qu'il y a sous ce rapport des rariations, qui ne peurent guère s'expliquer que par des différences dans la rapidité avec laquelle se développe ce premier commencement de bourrelet du manteau. Les Iarves des Hyaléacées observées par C. Vogt, qui oni perdu leur coquille, évidemment par suite de l'état maladif oì elles se trouvaient, et chez lesquelles la cavité d’invagination coquillière a encore reparu à ce moment, vieunent à l'appui de cette manière de roir. J'ai moimême observé cette réapparition tardive de l'invagination chez des larres malades de Cavolinia.

Toutefois la formation de cet enfoncement préconchylien me paraît trop répandue pour pouroir s'expliquer partout par des causes purement physiologiques; elle est surtout trop développée dans certains cas, tels que le Lymnée, d’après Lereboullet el Ganine, ct chez les Ptéropodes thécosomes, pour n’avoir pas une cause différente qui vient s'ajouter à la cause physiologique.

Ghez ceux des Lamellibranches que j’ai observés l'enfoncement est unique et donne naissance, en s'étalant, ¿̀ un bourrelet palléal en forme de 8 couché en travers. Ainsi se forme le premier rudiment de coquille, unique et silué sur la ligne médiane. Ce rudiment est, comme l'a déjà observé Leydig, composé d'une simple cuticule et se trouve posé sur la larve comme une selle sur le dos d'un cheval. Les sels calcaires se déposent ensuite dans les parties latérales, qui deviennent les valves, tandis que la partie de la coquille qui est situéc sur la ligne médiane reste mince, flexible et membrancuse et relie les valves entre elles. Chez le Dentale le mode précis de formation du tout premier rudiment de la coquille n'a pas élé observé, mais la suite du développement, qui a été si minutieusement décrite par de LacazeDuthiers, permel de supposer que les choses se passent ici comme chez les Lamellibranches, à cette seule différence près quue la coquille tout entière se durcit. Il n'y a dès Iors pas furmation de deux ralres 
et d'une charnière, mais formation d'une coquille en forme de cornet ouvert aux deux bouts. Ceci vient à l'appui du rapprochement, parfaitement juste, que de Lacaze-Duthiers a établi entre les Solénoconques et les Lamellibranches.

Les Gastéropodes pulmonés terrestres, dont le déreloppenent de la coquille a été décrit par Gegenbaur, sont un cas extrêmement intéressant. En effet, chez ceux dont la coquille reste interne pendant toute la vie (Limax), romme chez quelques-uns de ceux où elle devient plus tard externe (Clausilia), ce savant illustre a vu le premier rudiment de coquille apparaître dans une cavité située sous la peau, au point opposé à celui où se montre la bouche, carité close de toutes parts. J'ajoute que, d'après mes propres observations, cette cavité se forme par une invagination de l'ectoderme; cette invagination se forme et se clôt dans l'espace de vingt-quatre à trente-six heures et peut par conséquent facilement échapper à l'attention de l'observateur. Gegenbauir a suivi le développement de cette coquille interne chez Clausilia; il l'a vue grandir et prendre des dimensions considérables; il a vu la paroi de la cavité et la peau cui la recouvrait s'étirer, s'amincir, s'atrophier et finalement se déchirer à son centre. Et c'est ainsi que cette coquille, d'abord interne, finit par devenir externe. Mais Gegenbaur a le tort de vouloir étendre ces faits au genre Helix, où les choses se passent conformément au type général des Gastéropodes.

Ge type de développement de Clausilia diffère bien de celui des Céphalophores marins à coquille exterue, chez lesquels l'invagination se retourne avant de commencer à sécréter la coquille. Toutefois, j’ai vu, même chez ces derniers, la cavité d’invagination sécréter dans son intérieur des masses tuberculeuses chimiquement identiques au premier rudiment de la coquille. Ces masses étaient informes ct ne prenaient naissance que dans des cas pathologiques, lorsque la larve ne pourait arriver à se former une coquille suivant le mode normal. Néanmoins il est difficile de s'empêcher de faire un rapprochement entre cette sécrétion et celle de la coquille des Pulmonés terrestres.

En conclusion, roici les idées auxquelles ces romparaisons m’ont amené: La position du premierépaississement ectodermique, qui sécrétera la coquille, est illentique chez tous les Céphalophores et les Lamellibranches que l'on a étudiés sous ce rapport; elle est presque la même chez les Céphalopodes. Cet épaississement peut s'enfoncer el former une cavité d'invagination où une coquille interne est sécrétée; 
il peut s'enfoncer d'abord et puis s'étaler à la surface, ou bien il peut dès l'abord s'étaler sans s'enfoncer préalablement, et sécréter à sa surface le sommet d'une coquille exterrıe. Cet épaississement est l'origine première du bourrelet, qui accompagne le bord de la coquille externe et préside à sa croissance. Quel que soit le sort ultérieur de cet épaississement, il est strietement homologue dans tous les cas. La coquille interne des uns est comparable à la coquille externe des autres, puisqu'elles résultent toutes deux d'une sécrétion d'une mêmo région de l'embryon. Une coquille interne peut devenir externe par suite de la rupture des tissus qui la recouvrent. Dans les cas où il se forme une invagination qui ne se referme pas et qui s'étale pour former une coquille externe dès le premier abord, il est probable que cette invagination répond, non-seulement topographiquement, mais encore morphologiquement, à celle qui, dans d'autres cas, renferme la coquille interne; mais cette homologie est moins bien démontrée et demanderait à ètre prouvée par des transitions plus nombreuses.

Le mode de croissance de la coquille, surtout de la coquille larvaire des Ptéropodes, par l'addition d'anneaux superposés à son bord supérieur, mérite de fixer notre attention à eause des structures analogues qui ont élí décrites pour des genres fossiles de ce groupe. Nous avons vu que, chez plusieurs genres au moins, ces anneaux superposés constituent seuls la coquille, et qu'ils ne sont pas renforcés par des couches d'épaississement interne qui constituent la nacre chez la plupart des Mollusques. Les genres fossiles de l'époque paléolithique ont la même composition de la coquille par anneaux; ces anneaux présentent, du reste, deux arrangements différents suivant quel'annean inférieur embrasse celui qui lui est superposé (T'entaculites) ou qu'il est embrassé par lui (Cornulites). La cloison que Cavolinia et Hyalocylis forment après avoir abandonné leur coquille larvaire explique la présence, chez quelques coquilles fossiles de Ptéropodes, de ces cloisons transversales qui les ont fait classer par quelques auteurs parmi les Céphalopodes. L’ourerture que plusieurs de ces coquilles fossiles présentent à leur sommet pourrail bien être due à la fracture de la partie larvaire, c'est-à-dire de l'extrémité inférieure du test. Les lignes de petits enfoncements de la surface de la coquille chez Comularia rappellent les lignes également transversales de cavités chez Cavolinia et Hyalca.

L'abandon que fait l'animal du sommet de sa coquille, dans les 
genres où le corps devient épais et large à l'état adulte, s'explique par de simples raisons physiologiques, et se retrouve chez une foule de Céphalophores et de Céphalopodes, ainsi que l'a montré de Serres.

La présence chez quelques Céphalophores et Lamellibranches d'un stade où l'embryon se couvrirait d'une toison uniforme de petits cils, mérite tout notre intérêt, puisque ce stade correspondrait à peu près à la Gastriea de Hackel. Bes embryons ainsi couverts de cils ont été observés par trop d'auteurs, et chez un trop grand nombre de types divers, pour que leur existence puisse être révoquée en doute. Je dois faire observer, cependant, que je n'ai vu cette forme embryonnaire chez aucun des P'éroporles que j’ai étudiés, pas plus que chez les Hétéropodes; et pourtant ces animaux sont au nombre de ceux chez lesquels l'existence de cette toison caduque paraissait bien avérée. Ce duvet de cils n'est du reste que passager, et les auteur's qui l'ont vu rapportent qu'il tombe complétement avant la croissance des cils moteurs du voile. Chez Dentalium, nous savons, par les recherches de de Lacaze-Duthiers, que la larve jeune est couverte en majeure partie de cils; bien loin de tomber, ces bandes ciliaires se rapprochent les unes des autres, et forment par leur coalescence le bourrelet et les cils moteurs du voile. Chez les Ptéropodes, j’ai obser'vé quelque chose d'analogue à ce qui se passe chez. les Solénoconques. Les premiers cils apparaissent sur une zone assez large, qui se rétrécit el finit par former une simple rangée de cils moteurs portés par une seule rangée de cellules. Chez quelques Céphalophores prosobranches (Vermetus, Calyptrcea), le roile naît d’abord comme mamclons sur les côtés de la bouche, et s'étend de là autour de la région céphalique.

Le voile se retrouve chez les Lamellibranches, où il n'atteint pas la même extension que chez la plupart des Céphalophores. Les Céphalopodes paraissent en être complétement dépourvus. Il atteint de grandes dimensions chez quelques Gastéropodes marins.

J'ai trouvé, partout où je l'ai chcrchée (Ptéropodes, Hétéropodes et Gymnobranches), une bande de petits cils, située au-dessous de la rangée des cils moteurs et servant à amener à la bouche les particules nutritives, de la même manière que Claparède a décrite chez les Rotifères ${ }^{1}$. De Lacaze-Duthiers a observé cette préhension de nourriture chez le Verniet, mais sans indiquer la cause du phénomène.

1 Voir Claparède, Miscellanées zoologiques, Ann.sc. nat., 50 série, t. VIII, p. 5 et suiv., qui résume les données déx auteurs plus ancions sur ce sujet. 
L'analogie de fonctions el de position entre le voile des Mollusques et celui des Rotifères ne peut être l'objet d'un doute. Toutefois, avant de se prononcer sur leur homologie, il serait bon de mieux connaitre les relations de position du voile pendant la période embryonnaire avec les organes aroisinants et surtout arec les ganglions sus-œsophagiens de ces animaux, au inoment où ils se forment. Ces relations ne me paraissent pas ressortir d'une manière suffisamment claire de la description de Salensky.

La touffe eiliaire du sommet de Ia région céphalique, qui a été observée ehez beaucoup de Lamellibranches et chez Chiton, se retroure chez les Ptéropodes et paraît être représentée chez l'embryon de Brachionus, leseul Rolifère dont le développement soit connu d'une manière relativement complète, par un processus conique muni de cils.

Chez les Gastéropodes pulmonés, nous assistons à une réduction graduelle du voile, en commençant par les Prosobranches des eaux saumâtres, où il est encore bien déreloppé, pour diminuer chez les genres d'eau douce, tels que Planorbis et Ancylus, et disparaître entièrement chez les genres terrestres, tels que Limax.

Les organes de circulation larvaire sont encore plus répandus daus l'embranchement des Mollusques que le voile. Le type le plus eommun des Céphalophores marins est celui des Gymnobranches et des Ptéropodes, que j’ai retrouvé chez les Ilétéropodes. Il consiste dans la différenciation de deux régions du corps, qui deviennent particulièrement contractiles, celle de la base du pied et celle de la nuque (j'entends par le mot nuque le côté dorsal en dessous du voile). L'étendue de ces sinus varie beaucoup; nous avons vu que, chez les Hyaléacées, le sinus contractile du pied est très-petit, celui du dos très-étendu et limité seulement par le voile en avant et par le fond de la carité palléale en arrière. Chez Purpura et Buccinum, d'après Koren et Danielsen, chez Calyptrixa, d'après Salensky, ce sinus contractile dorsal se localise à la nuque, où il atteint un degré de différenciation plus complète. Chez les deux premiers genres de Prosobranches, il possède dans sa paroi, outre les fibres musculaires horizontales caractéristiques de ce sinus, des fibres verticales formant avec les premières une sorte de treillis.

Le sinus pédieux (résicule caudale) contractile des Limaces répond à une partie du sinus pédieux des Céphalophores marins, partie qui atteint un haut degré de développement. Le sinus céphalique des pulmonés terrestres est plus difficile à comparer. Salensky a par- 
faitement raison de se refuser à comparer ce sinus avec le sinus dorsal des Gastéropodes marins. Mais je ne puis donner raison au savant russe lorsqu'il cherche à identifier le sinus céphalique des preniers avec la région voilière de ces derniers. L'observation que les fibres musculaires sont transversales et non horizontales dans le voile, comme dans le sinus céphalique des Pulmonés, est parfaitement juste; mais, pour que ces deux parties fussent comparables, il faudrait qu'elles eussent les mêmes relations avec les organes avoisinants. Or le voile est une région circonscrite par un bourrelet cilié, et qui donne naissance dans son intérieur aux ganglions céphaliques, aux tentacules et aux yeux. Chez la Limace, j'ai vu les ganglions céphaliques se former par une invagination de l'ectoderme, de chaque còté au-dessous du sinus céphalique, au mème niveau que la bouche et que les tentacules. C'est encore à ce même niveau, mais un peu plus près du dos, que j’ai vu se former les vésicules oculaires; les otocystes résultent aussi d'une invagination de l'ectoderme au-dessous de l'endroit où se forment les ganglions céphaliques. Une autre considération fera encore mieux comprendre combien ce sinus est peu comparable à un voile. Chez Limax, en elfet, il s'étend, dès son origine, sur toute la portion qui sépare la bouche de l'invagination coquillière. Il répond donc topographiquement à toute la région dorsale des Céphalophores marins, et de plus ì la partie médiane de la région céphalique. J'ai dit topographiquement, mais non morphologiquement, car il s'agit ici non pas du développement différ'ent d'un organe homologue, il s'agit d'une région tout entière de l'embryon qui se façonne sur un autre modèle, qui se déreloppe dans unc autre direction.

Chez les Céphalopodes, l'on a observé aussi des sinus contractiles ; ils comprennent l'espace qui sépare l'ectoderme de la masse centrale de la hernie nutritive. Chez la Sépiole, j’ai vu les contractions se montrer surtout le long de la ligne médiane de ce sac. Ces sinus occupent une région comparable à celle qui forme la partie supérieure du pied des Céphalophores, et ne sont comparables à aucun des sinus contractiles de ces derniers.

Les homologies du pied des Mollusques et de ses excroissances ont donné lieu à bien des discussions. Sans vouloir trancher des questions, que les matériaux que j'ai à ma disposition ne suffisent pas même à éclairer d'une manière suffisante, j’indiquerai provisoirement les idées auxquelles les données déjà acquises m’ont amené. 
J'ai montré que le pied résulte d'une différenciation de toute la région ventrale, qui s'étend de l'anus jusqu’à la bouche de l'embryon. Il en est de même chez Limax et, d'après Lereboullet, chez Lymnæus. Gelte région forme une protubérance, de laquelle sortent les formations diverses que l'on comprend sous le nom de pied. Chez les Ptéropodes, cette protubérance s'aplatit de bas en haut et prend la forme d'une languette horizontale. Les parties latérales de la base de cette languette donment naissance aux nageoires, sans la moindre participation du roile à ces excroissances. J. Müller a déjà noté ce fait. Les nageoires poussent en haut et vers le côté dorsal, seulement jusqu'au niveau de la bouche, qu'elles ne dépassent guère en arrière. Quant à la partie médiane de ce pied, c'est elle qui porte l'opercule dans les cas oì celui-ci existe (C'ymbulia).

Chez les Pulmonés el chez beaucoup de Gastéropodes marins, la protubérance du pied s'aplatit, non pas de bas en haut, mais d'avant en arrière; il en résulte une large surface aplatie et parallèle à la face ventrale. L'opercule, qui répond toujours à la partie inférieure de la protubérance embryonnaire, se trouve occuper le côté dorsal de l'extrémité inférieure de ce pied en forme de fer à repasser. Faut-il conclure de là, comme on l'a fait, que cette extrémité inférieure du pied, que l'on a nommée pomatophore ou porte-opercule, répond à la languette des Ptéropodes? Certainement pas; la languelte des Ptéropodes n'est pas seulement un pomatophore, c'est le pied tout entier, condensé et peu développé.

Sourent le pied présente des lobes et des excroissances : tel est le cas, par exemple, de Dentalium. La lobure apparaît ici de bonne heure et divise, d'après de Lacaze-Duthiers, le mamelon pédieux en trois lobes, dont un médian et deux latéraux. Seulenıent cette division ne concerne que le sommet du mamelon, et, le pied venant à s'allonger, ces trois lobes se trourent séparés de la bouche par une longue portion cylindrique. Cette formation n'est certes pas comparable à celle des Ptéropodes.

Chez une foule de Gastéropodes, Pulmonés, Prosobranches, Opisthobranches et autres, le pied présente deux lobes plus ou moins marqués à son extrémité antérieure. Faut-il considérer ces lobes comme les homologues des nageoires des Ptéropodes? Pas davantage, car les nageoires répondent non pas à l'extrémité supérieure de la région pédieuse, mais aux parties latérales de cette région.

Les réphalopodes présentent dans cette même région plusieurs ren- 
flements dont les plus inférieur's deviennent le siphon, tandis que ceux qui se rapprochent davantage de la bouche deviennent des tentacules. Siphon et tentacules pris dans leur ensemble paraissent répondre au pied des autres Mollusques.

Le pied des Lamellibranches conserve une forme simple et peut aisément se déduire de la protubérance pédieuse des embryons de Géphalophores, si l'on suppose que cette bosse vienne à être comprimée latéralement, ou, ee qui revient au même, que son développement se fasse surtout dans le plan médian.

Chez les Rotifères enfin, du moins ehez Brachionus, le pied de l'embryon présente bien à l'origine les mêmes formes et les mêmes relations que eelui des Céphalophores, mais il se développe ensuite vers le bas et se bifurque à son extrémité.

Au lieu done de chercher à établir, par une simple comparaison des formes chez l'adulte, des homologies qui ne reposent que sur l'impression subjective de l'observateur, il me paraît à la fois plus simple et plus juste de dire, en s’appuyant sur les données embryogéniques :

Le pied est une différenciation de la région ventrale de l'embryon, qui se présente d'abord sous forme d'un disque épais, puis d'une protubérance. Ce disque a partout à peu près la même forme, la même extension, sauf chezles Céphalopodes. De ce disque naissent des parties de formes et de positions diverses, qui ne sont comparables entre elles qu'en tant qu'elles répondent aux mêmes régions du disque. Le siphon des Céphalopodes et le pomatophore des Gastéropodes répondent à l'extrémité inférieure du disque; ils sont done eomparables entre eux. La surface de reptation, qui se trouve chez la plupart des Gastéropodes, répond à la partie médiane et centrale du disque; elle répond donc au sommet de la languette des Ptéropodes. Les appendices filiformes ou en forme de lanière ciliée du pied des Hétéropodes et de quelques Ptéropodes (Gymbuliacées, Gymnosomes) naissent entre la partie qui porte l'opereule et le sommet de la protubérance pédieuse; ils sont donc comparables. Les nageoires des Ptéropodes, enfin, répondent à la partie latérale du disque embryonnaire; ils ne peuvent done se comparer qu'à une partie des tentacules des Céphalopodes, et à toute la partie latérale du pied des autres Céphalophores. Mais je fais en même temps celte réserve, que faire dériver deux organes à peu près des mêmes parties de l'embryon, ou bien les déclarer homologues, sont choses fort différentes. L'on peut admettre que le disque pédieux est homologue chez. les embryons de Céphalophores et de Lamelli- 
branches, sans que pour cela les organes si différents de forme, auxquels il dome naissance, soient strictement homologues entre eux. Etablir ces homologies n'est pas chose impossible; mais il faut pour cela des études préparatoires, des observations plus étendues et surtout plus précises que celles que nous possédons.

Les données que nous arons sur la formation du système nerveux chez les Mollusques sont trop incomplètes et trop clair-semées pour permellre de les généraliser. Chez Paludina et chez Limax le développement de la masse ganglionnaire sous-œsophagienne a été observé par Leydig et par Gegenbaur. Cette masse doil son origine à une simple différenciation d'une partie du tissu mésodermique du pied. Je me suis assuré par moi-mème de l'exactitude de ces observations en ce qui concerne le genre Limax. La seule donnée qui existe dans les travaux puhliés sur la formation de la masse ganglionnaire sus-œsophagienne est celle de Lereboullet, relative au Lymnée. Cel embryogéniste soigneux a vu une masse cellulaire apparaître à la face interne de l'ectoderme dans toute l'étendue de la région de la nuque et s'en détacher pour former les ganglions. Ray Lankester rapporte que la masse nerveuse se sépare de l'ectoderme chez Aplysia "dans lin région prostomiale " et suppose que cette masse derient l'anneau œsophagien tout entier; il n'en dit rien de plus. Mes propres obserrations sur les Ptéropodes montrent que les ganglions sus-øesophagiens naissent d'une double invagination de l'ectoderme dans la région céphalique. Chez Limax j’ai ru clairement ces masses sus-œesophagiemnes se former par une invagination de l'ectoderme de chaque côté, en arrière de la base des tentacules buccaux, et entrer ensuite en relation avec la masse sous-øesophagienne. Il paraît probable, d'après ces quelques données, que l'anneau œesophagien des Céphalophores naît de deux côtés à la fois, d'une invagination ou d'un dédoublement de l'ectoderme de la région céphalique d'une part, et d'une différenciation du mésoderme du pied d'autre part.

Chez les Céphalopodes, j’ai observé une double invagination de l'ectoderme en dedans des yeux, dounant naissance à un gros ganglion de chaque côté. D’après Ray Lankester, ces ganglions résulteraient " d'une diff́renciation des tissus qui entourent l'œsophage, " phrase qui nous apprend pen de chose. Le même auteur pense que l'invagination sitnée an-dessons des yeux chez ces animaux, donne naissance aux ganglions optiques, donnée dont j’ai démontré 
la complèle inexactitude ${ }^{1}$. D’après Ussow le collier wesophagien se formerait de trois côtés à la fois, en dedans des yeux, sur les côtés de l'œsophage et au côté ventral du cou, et cela par une simple différenciation du mésoderme; il se formerait done six masses ganglionnaires indépendantes les unes des autres; les deux supérieures donnant naissance aux ganglions optiques, les deux latérales aux ganglions sus-copophagiens, les deux ventrales anx ganglions sousnesophagiens. J'admets fort bien la possibilité de la fornution de ces derniers ganglions par différencialion du mésoderme au côté rentral, mais je crois m’être assuré que les invaginations que j’ai observées, el qui répondent aux ganglions optiques vus par Ussow, donnent naissance non-senlement aux ganglions optiques, mais cncore à toute la partie sus-œsophagienne du collier nerveux.

Chez les Rotifères, le système nerveux ne se compose que d'une masse ganglionnaire sus-œsophagienne, qui, d'après Salensky, se détache de l'ectoderme dans la région céphalique. Il serait à désirer que le point où cette masse se forme fùt précisé dans ses relations avec le voile et les parties voisines. En tout eas il suffit d'mn coup d'cil comparatif jeté sur ma figure $18, \mathrm{pl}$. IX, et sur les figures que donne K. Möbius de l'anatomie du Brachionus plicatilis pour s'assurer que la masse sus-cosophagienne des larves de Cymbulia est à un certain moment identique à la masse nerveuse sus-œsophagienne de ce Rotifère à l'élat adulte. La forme est la mème et les points d'attache latéraux sont les mêmes. Sous re rapport, comme sous beaucoup d’autres, les Rotiferes sont des larves permanentes des Mollusques céphalophores, et ont arec eux des rapports analogues à ceux qu'ont les Appendiculaires comparés aux Ascidies. A Salensky revient le mérite d'avoir attiré l'attention sur ces rapports en publiant côte à côte son étude embryogénique de Calyptraca et de Brachionus.

Les otocystes se forment de deux manières différentes. Chez les Ptéropodes ils se différencient au milieu d’une conche qui résulte d'un simple dédoublement de l'ectoderme. Chez les Céphalopodes, d'après les observations de Ray Lankester, de Grenacher, d'Ussow, et les miennes, ils se forment par une invagination de l'ectoderme. 11 en est demême chez Limax, d'aprèsl'étudeque j’ai faite sur le développenrent dece genre, et que je ne tarderai pas à publier. Je note que, chezles

- Voir ma Nole sur le déceloppement des Mollusques pleropodes et céphaloporles, dans ces Archives, t. III, no 3, p. ххин. 
P'éropodes, la région qui donne naissance aux otocystes se compose encore de cellules embryonnaires arrondies el très-grosses, au moment où cel organe des sens fait son apparition ; chez Sepiola et chez Limax l'ectoderme est déjà, en ce moment, composé de cellules extrêmement petites el de forme eylindrique. Cette différence dans la eonstitution de l'ectoderme dans les deux eas nous donne la clef des différences dans le procédé de formation de la vésicule auditive. Un simple dédoublement suffit, dans le premier cas, pour amener à la face interne de l'ectoderme une ou un petit nombre de grosses cellules embryonnaires qui, par leurs divisions ultérieures, s'arrangent aisément en une sphère crense. Dans le second cas, un simple dédoublement ne pourrait amener le résultat désiré ; il faut done que l'organisme ait recours à une invagination. Et e'est effectivement ce qui s'observe. Je note en passant que la eavité stomacale primitive se forme bien par invagination chez Pelagia, comme l'a décrit Kowalersky, tandis que chez Geryonia elle se forme par simple dédoublement du feuillet primitif. Or l'embryon de Pelagia se compose déjà de plusieurs centaines de cellules au moment où cette invagination a lieu, tandis que le dédoublement se fait chez Geryonia à une époque où l'embryon compte seulement trente-denx cellules.

L'on sait par les travaux de Grenacher, Ussow, et par le mien, que l'otolithe se forme chez les Céphalopodes au contact des cellules de la paroi de l'otocyste, et probablement dans le protoplasme de ces cellules. Il en est de même chez.Limax, et j'ai fait voir que chez les Ptéropodes l'otolithe apparaît dans l'une des cellules de la paroi de la vésicule. Ce mode de formation de l'otolithe pourrait bien être général chez les Mollusøues.

La cavité palléale varie de forme chez les diverses classes de Mollusques au point de n'avoir en commun que le nom et le mode de formation. Ce mode de formation est, du reste, très-caractéristique; la cavité branchiale résulte partout d'un repli de l'ectoderme qui se détache du corps et enveloppe, de la sorte, un espace compris entre ce repli et le corps. Ce mode de formation sépare nettement ces animaux de ceux appartenant, à mon sens, à l'embranchement des Chordés (Tuniciers et Vertébrés), chez lesquels la cavité branchiale est un dérivé de l'entoderme, une différenciation de l'entrée du tube digestif.

Chez les Ptéropodes et chez tous les Céphalophores que j'ai observés, la cavité palléale commence à se former à droite de l'anus. Elle se troure done du còté droil chez les types nì l'anus est sitné au milieu 
de la face ventrale; elle recule vers le dos dans les cas où l'anus se forme à droite. Dans tous les cas, elle est asymétrique. Chez les Lamellibranches, les Solénoconques et les Céphalopodes, au contraire, elle est, du moins à l'origine, parfaitement symétrique.

L’on sait que les Lamellibranches, les Solénoconques et les Céphalopodes sont symétriques peudant toute la durée de leur vie (les quelques exceptions à cette règle étant dues à des phénomènes d'adaptation). Les Céphalophores sont asymétriques déjà à l'état de larves et pendant le reste de leur vie. Chez tous cependant, l'embryon est rigoureusement symétrique et la torsion ne commence à se manifester qu’à la période larvaire. Les Ptéropodes se rapprochant beaucoup de la symétrie à l'état adulte, dans leur forme extérieure du moins, il élait intéressant de savoir si cette symétrie apparente était un retour au type, ou si ces animaux passeni d'abord par un état asymétrique pour se redresser par la suite du développement individuel. J'ai montré que les larres des Gymnosomes et des Thécosomes campyloconques sont aussi asymétriques que celles de n’importe quel Gastéropode. Les larves des Thécosomes orthoconques conservent pendant assez longtemps la symétrie primitive; ils commencent parfois à s'en départir dès l'apparition du sommet de la coquille, qui est un peu déjeté de côté chez les Styliolacées. L'embryon se courbe par suite du développement plus rapide de son côté droit; il a donc une tendance à l'enroulement senestre. J'ai montré que les otocystes et, généralement parlant, tous les organes de la larre se développent plus vite du côté droit. C'est aussi de ce côté qu’apparaît la cavité palléale, et, dès ce moment, l'asymétrie devient évidente; l'anus et l'intestin sont rejetés à gauche, le rein apparaît d'un côté et le cơur de l'autre. Mais la coquille continue à croître toute droite, ou, si elle se courbe un peu, e'est dans le plan médian.

Au point de vue phylogénique, les Céphalophores seraient done des animaux à plan symétrique qui se sont enroulés et ont perdu par là leur symétrie. Les Ptéropodes, commie les autres Céphalophores, ont la symétrie originelle suivie d'asymétrie; puis ils se redressent et regagnent, mais seulement en partie, et pour la forme extérieure, leur symétrie première.

Les différences que présentent les larves des divers tỵpes de Ptéropodes, sont plus grandes que celles qui les séparent des élats larvaires des autres Géphalophores. Les caractères embryogéniques ne 
suffisent done pas à justifier la séparation de ce groupe, comme ordre distinct, du reste des Céphalophores, el si je laisse subsister cette séparation, e'est your des raisons tirées de la comparaison des animaux adultes.

\section{RÉSULTATS PIINCIPAUX.}

Le vitellus des Ptéropodes avant la fécondation est histologiquement une cellule simple arec dépôt de matière nutritive dans son intérieur. Le vitellus fécondé est dépourvu de membrane el de nueléus. Il se constitue d'une partie formative ou protoplasmique et d'une partie nutritive composée d'un réseau de protoplasme dans les mailles ducquel se tronvent les globules nutritifs. Au centre de la partie formative se trouve une étoile constituće par les granules du protoplasme, rangés en lignes droites divergentes. Les rayons de cette étoile ront jusqu'à la limite de la partie formative et les globules nutritifs eux-mêmes s'arrangent en lignes.

Après la sortie du corpuscule dit de direction, un nucléus apparaît au centre de l'étoile, qui s'efface à mesure que le nucléus grossit. Les granules et les globules du vitellus cessent d'être en lignes. Avant chaque segmentation le nucléus disparaît pour être remplacé par deux étoiles moléculaires qui prennent naissance dans son intérieur. Le centre de chacune de ces étoiles peut être considéré comme un centre d'atiraction; tonte la substance vitelline obéit à cette attraction. Après la segmentation un nucléus reparaît au milieu de chaque étoile et la substance vitelline rentre en repos.

Le résultat de la segmentation, qui ne diffère que peu du type connu pour les Gastéropodes, est le développement d'une moitié nutritive composće de trois grosses sphères et d'une moitié formative composée de sphérules transparentes. Les cellules nutritives se divisent, ensuite, produisant une couche superficielle de pelites cellules, qui achèvent d'envelopper les trois grosses sphères nutritives et constituent l'ectoderme. La quatrième des grosses sphères centrales, uniquement composée de protoplasme, se divise complétement et donne naissance à un épaississement de la couche ectodermique. Cette région correspond à l'extrémité inférieure de la larre. La ligne de rencontre des trois sphérnles nutritives coïncide avec l'axe oralaboral de la larve. L'ectoderme se referme en dernier lieu au point rie rencontre des trois sphérules, point qui doit coïncider soit avec le 
pôle aboral, soit avec le pôle oral de la larve. C'est pour cette dernière alternative que je me prononce.

Le développement embryonnaire des Gymnosomes établit la transition entre celui des Thécosomes, que je viens de résumer, et celui des Hétéropodes, entre la formation des feuillets embryonnaires par enveloppement et la formation par invagination.

La cavité digestive se forme par une simple différenciation de la masse des cellules nutritires ou centrales. Il en résulte une carité fermée de toutes parts et trilobée. Le lobe médian donne naissance au tube digestif, les lobes latéraux aux sacs nutritifs. Les cellules composant les parois de cette cavité descendent directement des cellules nutritires ou centrales de l'embryon; elles sont petites et nombreuses autour de la cavité médiane, cunéiformes et composées en majeure partie de substance nutritive autour des cavités latérales. La partie médiane s'allonge pour former l'estomac et l'intestin. Une invagination de l'ectoderme, partie du point où ce feuillet s'est refermé, s'enfonce à la rencontre de l'estomac, arec lequel elle se soude. Cette invagination répond à la bouche et à l’œsophage; le point de soudure, au cardia. Elle présente en avant un diverticule qui donne naissance à la radule. Ce développement du tube digestif correspond point par point à ce que l'on sait du développement des Rotifères. - L'extrémité de l'intestin s'ourre à l'extérieur sur la ligne médiane de la face ventrale chez les Ptéropodes orthoconques, du côté droit chez les Campyloconques et les Gymnosomes.

Les larves ne se couvrent jamais d'une toison continue de cils vibratiles. Les premiers cils qui apparaissent sont les cils moteurs; ils sont implantés par petites houppes sur une zone circulaire, qui se trouve à peu près au niveau de la bouche. Puis ils se mettent tous sur un rang et une bande de petits cils naît au-dessous des gros cils moteurs, servant à amenel les particules nutritives jusqu'à la bouche. Le voile est muni de fibres musculaires ramifiées. C'est un organe purement larvaire et transitoire.

Le pied a son origine dans un épaississement de l'ectoderme, qui occupe la plus grande partic de la face ventrale de l'embryon. Il prend ensuite la forme d'une bosse, puis celle d'une languette horizontale, qui porte parfois un opercule ì sa face inférieure. Il se divise en un lobe médian et deux lohes latéraux qui deviennent les nageoires. Il répond au pied tout entier. Les nageoires répondent aux parties latérales du pied des autres Céphalophores. 
Les muscles résultent d'un dédoublement partiel de l'ectoderme.

La cavité palléale se forme par enfoncement de l'ectoderme entre le bord de la coquille et le eou de la larve, toujours à droite de l'anus, quelle que soit la position de ce dernier.

Les larves de Ptéropodes ont deux sinus contraetiles, situés l'un an pied et l'autre dans la région dorsale, qui se renvoient le liquide contenu dans la cavité du eorps. Ces sinus ne doivent leur existence qu'à la différenciation de fibres musculaires transversales au côté interne de l'ectorlerme de ces régions. Ni l'un ni l'autre de ces sinus ne peut se comparer à ceux de l'embryon de Limax. Le sinus céphalique de la Limace répond à tonte la partie médianc du voile el à toute la région dor'sale des embryous de Ptéropodes. Le sinus contraclile du pied des Limaces se trouve à l'extrémité et non à la base du pied, comme chez les Ptéropodes.

Le rein se forme aux dépens de l'eetoderme, le cœur par la différenciation d'un amas de cellules du mésoderme. L'ouverture interne du canal rénal débouche en dehors du eccur et s'ourre dans le péricarde lorsque celui-ci vient à se former plus tard. Le rein bat avec presque autant de vivacité rque le coeur. L'aorte et les artères se forment par la différenciation de cordons de cellules mésodermiques.

Les parois de l'estomac se différencient en deux couches, une couche externe de fibres musculaires et une couche muqueuse interne; cette dernière produit cinq dents cornées, précédées parfois de l'apparition d'une plaque larvaire unique. Les sacs nutritifs, au nombre de deux dans l'origine, se soudent en un seul chez les Orthoconques. Ce sac, qui s'ourre dans la partie dorsale de l'estomac, se résorbe et diminue rapidement ehcz les Hyaléacées; il se développe, au contraire, chez les Styliolacées et les Créséidées, où il parait joucr provisoirement le rôle de foie. Il diminue, dans tous les cas, à mesure que lc foie se développe. Lc foie se compose de petits diverticules de la paroi de l'estomac.

Les otocystes se forment de bonne heure, au milieu d'une couche qui résulte d'un dédoublement de l'ectoderme, encore composé de grosses cellules embryonnaires. L'otolithe prend naissance dans l'épaisseur de la paroi de la vésicule, pour tomber plus tard dans sa cavité. Chez les Limaces et les Céphalopodes, l’otocyste se forme par une invagination de l'ectoderme, déjà composé de très-petites cellules cylindriques. La grosseur des cellules embryonnaires de la couche génératrice paraît être, dans ce cas, comme dans beaucoup 
d'autres, la cause qui clétermine le mode de formation d'un organe par invagination ou par simple dédoublement.

Le système nerveux se compose d'une masse nerveuse céphalique et d'une masse sous-osophagienne. La première se forme par une double invagination de l'ectoderme de la région céphalique dans le champ circonscrit par le voile; le mode de formation de la seconde n’a pas été observé chez les Ptéropodes. La forme de la masse nerveuse céphalique de la larve dle Ptéroporle est la même que celle du ganglion des Rotifères adultes.

L'apparition de la coquille est précédée par la formation d'une invagination de l'ectoderme, un peu en avant du pôle aboral. Cette invagination coquillière ou préconchylienne se retourne et le premier rudiment de la coquille apparaît sur la saillie ainsi formée. Dans des cas exceptionnels ou anormaux, cette invagination ne se retourne pas, ou bien se reforme après avoir disparu; son existence et celle d'une coquille externe s'excluent. Elle est le point de départ du bourrelet qui sécrète la coquille anneau par anneau, et qui devient le bord du manteau. La première partie de la coquille, celle qu'habite la larve, diffère souvent de la partie qui vient s'y ajonter plus tard; elle peut persister, tomber ou se casser, et m'a fourni des caractères qui m'ont permis de subdiviser le sous-ordre des Ptéropodes thécosomes. L'existence de l'invagination préconchylienne ne s'explique pas d'une manière satisfaisante par des causes purement physiologiques; elle parait donc avoir des causes héréditaires et peut morphologiquement se comparer à l'invagination coquillière des Mollusques à coquille interne, invagination que j’ai étudiée chez la Sépiole et la Limace. L'existence et la signification de cette invagination chez les Céphalophores, les Céphalopodes et les Lamellibranches ont été graduellement débrouillées par Lereboullet, Semper, Salensky, Ganine, Ray Lankester el moi-même.

Les produits sexuels prennent naissance autour du sac nutritif et aux dépens de l'entoderme. La scxualité ne peut donc être attribuée qu'à un feuillet embryonnaire chez les Ptéropodes.

SUPPLÉMENT.

Mon mémoire sur le développement des Ptéropodes était à l'impression, lorsqu'un nouveau séjour à Messine pendant le mois de 
février et de mars $1875^{\circ}$ me fournit l'occasion de vérifier ceux de mes résultats qui étaient restés plus on moins douteux.

Et tout d'abord, je m'occupai de suivre les premières phases du développement jusqu'au moment où apparaît la bouche, pour déterminer à nouveau la position que nous devons donner à cet organe, comparativement aux sphérules de segmentation el au point de départ du feuillet externe. Celle étude nouvelle m’a démontré que la houche se forme bien effectivement an pôle nutritif, et m'a fait comprendre la cause de l'erreur dans laquelle j’étais tombé en premier licu, lorsque je plaçais cet organe dans le voisinage du pôle formatif. En effet, les corpuscules de rebul (autrement dits corpuscules de direction) se trouvent dans le voisinage de la bouche au moment où apparaît cet organe dans tous les cas où ces corpuscules sont encore restés adhérents à l'ectoderme à ce moment-là. A l'origine, ils étaient situés au pôle formatif, plus tard on les retrouve dans le voisinage de l'orifice buceal; élant, pour d'autres raisons, arrivé à reconnaître que cet orifice se forme au pôle nutrilif, il paraissait naturel de conclure que les corpuscules de rebut s'étaient détachés de l'ectoderme et araient royagé ; mais il n'en est rien, ils restent souvent fermement adhérents au fenillet externe et ne peuvent rlans ce cas avoir voyagé d'une manière indépendante.

La solution de cette énigme m’a été donnée par l'étude du développement des Hétéropodes, et cette solution s'est trouvée aussi applicable aux Ptéropodes, ainsi que je m’en suis assuré par l'observation directe. En effet, aussitôt que les deux feuillets primitifs de l'embryon sonl formés, le feuillel externe se met à croître et à s'étaler beaucoup plus rapidement d'un côté que de l'autre. Ge côté à croissance rapide répond à la région rentrale et postérieure de la larve, à celle où se trouve, on se le rappelle, la sphérule primitive protoplasmique, à celle qui donne naissance au pied et au manteau. Le tissu ectodermique qui occupait dans l'origine le pôle formatif subit de la sorte un déplacement relatif et paraît remonter le long du dos de l'embryon pour arriver enfin à la région céphalique.

La rapidité de ce déplacement est beaucoup plus grande chez les Pléropodes que chez les Hétéroporles, gràce à l'accumulation de protoplasme qui se produit, chez ceux-là, dès les tout premiers stades de développement, ì la partie qui répond au pôle postéricur de l'embrỵon. Cette rapidité même marait dérouté et m’avait empêché de saisir complétement la signification des faits observés. 
Ainsi donc la posilion de la bouche, chez ces embryons à segmentation totale, modifiée par la présence d'un vitellus de nutrition considérable, répond tout à fait à ce qu’elle est chez les Hétéropodes, où les feuillets se forment par le procédé de l'invagination.

Je remarque encore que les trois grosses sphérules nutritives des Ptéropodes montrent, pendant la formation du feuillet externe, non-seulement un déplacement de leur partie protoplasmique, mais encore une sorte de rotation lente et pęu considérable de chaque sphérule, accompagnéc d'un déplacement de ces sphérules entre elles. Ce fait ressort du reste d'un examen attentif des figures 12 à 17 de la planche II.

Un autre point méritait d'être étudié à nouveau, à savoir : l'origine première des produits sexuels. Depuis mon retour de Messine j'arais pris connaissance de l'important travail d'E. van Beneden sur ce sujet. J'avoue que les généralisations s'étendant à tout le règne animal, auxquelles se livrait le savant belge en s'appuyant sur une base aussi mince, me parurent inadmissibles. Cette base consistait seulement en une série d'observations, fort soignćes du reste, sur deux genres de Polypes hydraires. E. van Beneden s'appuyait, il est vrai, sur des travaux qui font provenir, chez les Vertébrés, le testicule du corps de Wolf, et celui-ci du feuillet externe; l'ovaire du repli pleuro-péritonéal, qui dépendrait de l'entoderme; mais l'on sait à quel point les données relatives à l'origine première du feuillet moyen du corps de Wolf et du testicule des embryons de Vertébrés sont encore contradictoires et incertaines.

La lecture du dernier mémoire de J. Müller (cxix) sur le déreloppement des Ptéropodes fit encore mieux sentir la nécessité de revoir ce point. Le grand anatomiste décrit un corps pyriforme qui pend à côté de l'intestin, chez les larves de Creseis, et ce corps donnerait, selon lui, naissance aux organes génitaux. Je pouvais d'autant moins douter de l'existence de ce corps qu'il était indiqué sur plusieurs de mes dessins, quoique je ne lui eusse pas accordć une attention spéciale. Mais, d'un autre côté, j’arais vu, à n’en pas douter, la glande génitale se détacher de la partie de l'entoderme qui constitue le sac nourricier de ces larves. M'élais-je trompé? arais-je pris la partic pour le tout, el la glande génilale avait-elle réellement une origine double?

Résoudre cette question n'était pas chose difficile; le matériel du moins abondait à Messine le printemps dernier. Chaque coup de ma coiffe traînante, donné dans des profondeurs de cinq à dix brasses, 
ramenait une multitude de larves de C'reseis à tous les stades du déreloppement larvaire et ultćrieur, depuis les plus jeunes larves jusqu'à l'adulte.

Le corps pyriforme de Miiller oceupe bien, chez des larves avancées, la position que lui a assignée cet excellent observateur (voir pl. VI, lig. $4^{*}$ ). Il se trouve donc du côté gauche, à la hauteur du cœur et du sommet du sac nourricier, et affecte plutôt la forme d'un fuseau que celle d'une poire. Il est plongé dans le liquide qui remplit la cavité du corps et libre sur lout son pourtour, sauf à son extrémité supérieure et au côté qui est tourné vers l'ectoderme, qui tapisse intérieurement la coquille. En effet, il est relié à l'ectoderme coquillier par une série de prolongements sarcodiques de son parenchyme, el. un filament de même nature, mais plus gros et plus long, relie son extrémité supérienre avec le rectum et la paroi de la cavité palléale dans le voisinage de l'anus. Tous ces filaments sont plus gros à leur point de départ du corps pyriforme qu'à leur extrémité opposée, qui est ramifiée et s'insère à l'ectoderme par une multitude de fibrilles d'une ténuité extrême. Histologiquement parlant, ce corps se compose d'un assez grand nombre de petites cellules peu distinctes les unes des autres, mais munies chacune d'un nucléus.

Ghez les plus jemnes larves, le corps en question est très-petit, globuleux, composé seulement d'une demi-douzaine de cellules et trèsrapproché de l'anus. Ses connexions sont celles que nous venons de voir, seulement les filaments d'attache sont moins nombreux et beaucoup plus courts. Le filament qui s'insère dans le voisinage de l'anus est beaucoup plus gros'que les autres. Il est done très-probable que ce corps provient de l'eetoderme, d'autant plus que l'on sait que les larves de beaucoup de Gastéropodes, celles des Gymnobranches en particulier, possèdent à côté de l'anus deux corps cellulaires provenant de proliférations de l'ectoderme. L'un de ces corps donne naissance au rein; l'autre ne peut guère être autre chose que l'origine du corps pyriforme. Connaissant l'origine et la structure de l'organe, il ne restait plus qu'à en suivre le sort ultérieur.

Mes séries de larves et de jeunes étaient assez complètes pour me permettre d'arriver facilement à une certitude absolue à cet égard. Le corps pyriforme, arrivé au point où il est représenté sur la figure 4, pl. Vl, se met à croître très-rapidement. Il s'allonge en arrière, et vient s'accoler au côté gauche de la partie supérieure du sac nourricier (pl. VI, fig. $3, G h$ ). Ses connexions avec l'ectoderme disparaissent 
à mesure que sa liaison avec le sac nutritif devient plus intime. Pendant ce temps, les cellules de son parenchyme se sont beaucoup multiplićes, et ont fini par prendre l'aspect de petits globules de protoplasme qui s'aplatissent nutuellement et ne montrent plus de nucléi distincts. La surlace de l'organe prend un aspect lobé, et chacun de ces lobes arrondis présente intérieurement une vacuole de forme irrégulière, plus on moins étoilée. L’organe tout entier est atténué par le bas, où il se termine presque en pointe, élargi par le haut, où il enveloppe la moitié gauche du sommet du sac nourricier. Il a done l'aspect d'un triangle isocèle dont l'angle le plus aigu est tourné vers le bas. Il continue ensuite à croître et à s'allonger, mais moins rapidement. Sa texture interne se modifie petit à petit; de transparent, il devient de plus en plus opaque, et l'on reconnaît sans peine que les petits globules qui constituaient le parenchyme ont domé naissance à des faisceaux de spermatozoaires. C'est, àn'en pas douter, le testicule.

L'oraire se forme de la manière que j’ai déjà décrite (voir pl. VI, fig. 3 , 4 et $8, G h)$. Les cellules jaunâtres de la paroi du sac nourricier, sur tout le côté droit de l'organe, se différencient chacune en une partie externe incolore $\mathrm{ct}$ ! une partie interne jaunàtre. Puis chacune d'elles sc scinde par le travers; d'où résulte un dédoublement de la couche unique de cellules du sac nourricier en une couche jaune qui continue à former la paroi de cet organe, et une couche incolore qui lui est accolée extérieurement. Les cellules de cette dernière couche se divisent d'abord entre elles, puis grossissent, et chaque cellule devient un ovule. L'ovaire n'arrive à maturité qu'assez longtemps après le testiculc, et à un moment où ce dernier est à peu près vidé. Noll-seulement il prend naissance plus tard que l'organe mâle, mais encore il ne croît que plus lentement.

Les deux moitiés de la glande hermaphrodite sont d'abord séparées par toute l'épaisseur du sac nourricier, et ne se touchent que par leurs bords. A mesure qu'elles mûrissent le sac diminue, et elles finissent par ètre accolées et soudées sur toute leur longueur; mais elles restent toujours l'une à droite, l'autre à gauche, et ne s'enveloppent pas, ne se mêlent pas aussi complétement que chez d'autres Céphalophores.

Ce qui m'avait précédemment induit en erreur sur l'origine du testicule était une lacune dans mes séries de jeunes exemplaires, jointe à la rapidité de la croissance du corps pyriforme, précisément 
pendant la période correspondante. N'ayant pas d’intermédiaires entre les états représentés sur les figures 3 et 4 de la planche VI, et ne pouvant guère admettre que le testicule considérable de l'un pût provenir du petil corps pyriforme de l'autre, j’avais élé amené à croire que le testicule, de même que l'ovaire, naissait par scission de la paroi du sac nourricier. Cette hypothèse paraissait d'autant plus plausible que les deux moitiés de la glande sont déjà soudées par leurs bords sur la figure 3 .

Mes nouvelles observations sont une confirmation aussi claire qu'inattendue des idées théoriques de van Benedeu, confirmation d'autant plus digne d'attention, que j'étais plus opjosé à ces idées, lorsque je me remis à l'étude de ce sujel. Néanmoins, encore à l'heure qu'il est, je ne saurais considérer cette origine des produits mâles dans le feuillel externe, et des produils lemelles dans le feuillet interne, que comme un fait peut-ĉtre très-répandu, mais dont la généralité pour fout le règne animal est moins qu’hypothétique.

Enfin, relativement ausac nourricier, l'étude des jeunes des genres Creseis et Sprialis m'a montré que cet organe n’a pas chez tous les Ptéropodes une fonction aussi exclusive que chez les Hyaléacées. Chez Creseis, en effet, les cacums, qui sont l'origine première de la glande hépatique de l'adulte, naissent, non pas aux dépens de la paroi de l'estomac, comme chez C'avolinia et chez Hyalcea,mais bien sur le pourtour de l'extrémité supérieure du sac nutritif. C'est donc le canal eflérent de ce sac qui donne naissance au foie, tandis que sa partie inférieure, comprenaut les dix-neuf vingtièmes de l'organe, tombe plus ou moins en dégénérescence. Chez les Campyloconques, la portion du sac qui persiste, en se métamorphosant et donnant naissance à la glande acineuse du foie, est déjà un peu plus grande. Chez les Hétéropodes, au contraire, le sae tout entier forme des lobes et se mélamorphose en foie.

Sous ce rapport, comme pour la blastogénèse, les Hyaléacées sont un extrême qui est relié par des transitions nombreuses à l'autre extrême, représenté par les Hétéropodes et les Gymnobranches. Ces transitions nous sont fournies par les Ptéropodes mêmes, surtout par les Campyloconques el les Gymnosomes. 


\section{EXPLICATION DES PLANGHES}

PLANCHE I. - Cavolinia tridentata.

Fig. 1. Partie d'une chaîne d'œuf́s fraîchement pondus. Le moreeau représenté com prend deux renflements et le commencement d'un troisième. Dessin fait à la chambre claire et terminé d'après natıre sous un grossissement de 5 diamètres.

Fig. 2. Portion d'un renflement de la même elâine, dessiné de même sous un grossissement de 10 diamètres.

Fig. 3. Un vitellus vivant et fraîchement pondu, an moment où le corpuseule excrété, $x$, vient de sortir et montrant les deux portions protoplasmique et nutritive du vitellus, aiusi que l'arrangement régulier des granules du protoplasme ei des globules nutritifs. Dessin fait à la elıambre claire, à un grossissement de 250 , et terminé d'après nature, à Messine, le 21 avril 1874.

Fig. 4. La partie formative d'un vitellus vivant au moment où le corpuscule va sortir, montrant l'étoile moléculaire du protoplasme. Dessin fait à la ehambre elaire; grossissement, 230 .

FıG. 5. Deuxième stade. Un œuf vivant après la première segmentation, au moment où les nouveaux nucléi réapparaissent, montrant l'inégalité de grosseur et de composition des deux sphérules. Dessin fait à la chambre claire, à un grossissement de 150,7 mai 1871.

F1G. 6. Deuxième stade. Le vitellus se prépare ì la seconde segmentation. Il est eneore composé de deux sphérules, mais chaque sphérule possède deux étoiles moléculaires. Préparation à l'acide acétique. Dessin fait à la chambre elaire et fini d'après nature. Grossissement, 150. Messine, 7 mai 1871.

Fig. 7. Troisième stade. Le vitellus vivant segmenté en quatre, vu par le póle formatif. Dessin lait à la chanbre claire et terminé d'après nature. Grossi 150 fois. 23 avril 1871.

Fıg. 8. Quatrième stade. Le vitelius vivant segmenté en huit, vu par le pôle formatif. Dessin fait à la chambre claire, à un grossissement de 130 , et terminé d'après nature à Messine. Même date.

FıG. 9. Cinquième stade. Le vitellıs vivant, fractionné en douze, vu par le côté formatif. Les cellules de quatrième génération ont chacune déjà leur nucléus Dessiné à la chambre claire, ì un grossissemeut de 150. Miême date.

Fı. 10. Sixième stade. Le vitellus vivant, peu d'heures après la ponte, vu par le côté formatif. Dessin la.t à la chambre claire, grossi 150 fois. Nềme date.

Fig. 11. Septième stade. Le vitellus virant, vu par ie pôle formatif, montrant la formation des quatre cellules de septìme génération. Dessiné à la chambre elaire, à un grossissement de 150. Mîme date.

PLANGHE II. - La mème espèce.

FIG. 12. Septième stade. Le vitellus vivant, vu par le pôle nutrilif, montrant la ma nière dont la sphérule protoplasmique primitive s'est divisée. Dessin fait à la chambre claire et grossi 130 fois. Même date. 
Les fignres 13-18 ont toutes été dessinées à la chambre claire, ì un grossissement de 150 diamètres, d'après cles œufs vivants; les dessins sont tous datés de Nessine, en mai 1871, mois pendant lequel la température était extrêmement élevée, ce qui explique le développement rapide de ces œufs. Les figures 12-16 ont été tontes dessinćes dans la nuit du 29 au 30 mai.

Flg. 13. L'ébauche embryonnaire, ảgée de sept heures cnviron, vue par le côté lormatif et un peu par le còté céphaliqne, montraut la manière dont se forme l'ectoderme.

Fig. 14. L'ébauche embryonnaire, vue par le côté opposé, âgée de six lieures.

likg. 15. La même que celle de la figure 13 , même âge, vue par le pôle nutritif.

Frg. 16. L'ébauche, vue du côté nutritif, âgée de huit heures.

FIG. 17. L'ébauche, vue obliquement par le còté céplıalique et nutritif, âgée de douze à quinze heures; dessin combiné d'après deux dessins faits à la chambre claire, d'après le virant.

Fig. 18. Embryon de vingt-quatre heures, vu par le côté formatif et inférieur. L'ectoderme est représenté en coupe optique.

Fig. 19. Larve vivante de quarante-buit heures, le 6 mai 1874, vue par le côté droit. Chambre elaire ; grossissement, 200.

Fig. 20. Larre un peu moins développée, traitée par l'acide acétique, vue par le côté dorsal, le 9 mai 1874. Chambre elaire; grossissement, 200.

Fig. 21. Larve pareille à la précédente, traitée de la même manière, vue par le côté ventral.

Fıg. 22. L'invagination préconchylienne d'une larve âgée de quatre jour's, ell mars 1874, par une températnre très-basse; dessinée d'après le vivant à la chambre claire; grossissement, 200. La partie interne de l’invagination est représentée en coupe optique; vue par le côté droit.

Fig. 23. Comme la précédente, sauf que le sac intérieur est représenté en entier.

Fig. 24. L'invagination préconchylienne d'une larve vivante de trente-huit heures, vue par le côté droit, clessinéc sans l'aide de la chambre claire, au moment où elle se prépare à se retourner; le 8 mai 1874.

Frg. 25. La même, quelques minules plus tard.

FIG. 26. L'otocyste, dessiné sans chambre claire, avant l'apparition de l'otolithe.

Fig. 27. Le même, un peu plus développé et traité à l'acide acétique.

FIG. 28. L'otocyste et les deux couches extérieures, ectoderme et mésoderme d'une larve vivante, âgée de six jours, en mars 1874; grossissement, 300.

PLANCHE III. - La même espèce.

Fig. 29. Larve vivante, vue par la face ventrale et un peu par la droite, âgée de quarante-trois heures. Messine, fin mai 1871. Dessin fait à la chambre claire à un grossissement de 1500.

Frg. 30. Larve un peu plus âgée, vue par le dos et un peu par l'extrémité céphalique, montrant le commencement de l'invagination nerveuse. Fin mai 1871. Chambre claire; grossissement, 150.

FIG. 31. Larve un pen plus avancée que la précédente, même aspect à peu près. 
Dessin réduit ì un grossissement de 150 , d'après un dessin à la chambre claire. Messine, 20 mai 1874.

FlG. 32. L'invagiuation nerveuse d'nne larve vivante de trois jours et demi, 19 mai 1874, vue par le côté dorsal, dessinée sans l’aicle de la chambre claire, à un grossissement de 20 ellviron.

Flg. 33. La même, vue par le còté, grossie un pen plus fort; même âge et même date.

Fig. 34. Les deux cellules anales d'une larve vivante, àgée de trois jours trois quarts, vues un peu par la droite; dessin fait à main levée et grossi onviron 400 fois.

Frg. 35. Le voile et l'otocyste de droite d'une larve vivante, arrivée à peu près au même point de développement que les précédentes; dessin fait sans chambre claire. Mars 1874.

Fıg. 36. Larve traitée par l'acide acétique, àgée de deux jours trois quarts, le 27 avril 1874, mais pas plus avancée que celle de la figure 29; vue par la face ventrale et un peu par la droite, en coupe optique. Chambre claire; grossissement, 150.

FIG. 37. Larve vivante, âgée de trois jours trois quarts, 10 mai 1874, vue par le côté ventral et la droite, montrant la formation du cœur, du rein et de l'intestin. Chambre elaire ; grossissement, 150.

Fig. 38. Larve vivante, âgée de trois jours trois quarts, 3 juin 1871, vue par le côté droit. Chambre clairc; grossissement, 150.

Fig. 39. La mème, vue par le côté gauche et dorsal; même grossissement.

Fig. 40. Le pied d'une larve an même point de développement que celle de la figure 29, vu par le côté céphalique. Chambre claire; grossissement, 150.

FIg. 41. Le pied d'une larve pareille à celle de la figure $3 \mathbf{j}$, mème aspect, même grossissement que la figure précédente.

FıG. 42. Le pied d'une larve pareille à celle de la figure 37, toujours vu d'en haut. Même grossissement.

\section{PLANCHE IV. - Mème espèce.}

Fig. 43. Un exemplaire jeune, âgé de quatre jours trois quarts, le 4 juin 1871, peu après la métamorphose, vu par la face dorsale. Dessin fait ì la chambre claire, d'après le vivant, à un grossissement de 150.

Fig. 44. Le même, vu par la face ventrale; mème grossissement.

FIG. 45. Un jeune, âgé de six jours et demi, le 3 juin 1871, ru par le côté droit, réduit à un grossissement de 75 , d'après un dessin fait à la chambre claire.

Fıg. 46. Un jeune, pris en mor le 26 juin 1871, vu par le côté dorsal; dessin réduit it un grossissement de 20 .

F1G. 47. Le bord latéral de la coquille larvaire d'un jeune de einq jours, grossi 200 fois.

FIG. 48. Larve de trois jours trois quarts, le 10 mai 1874, vue par l'extrémité céplalique. Dessin fait d'après le vivant à la chambre claire, grossi 200 fois.

PLANCHE V. - Hyalocylis striata.

Fig. 1. Une chaîne d'œufs, nouveliement pondus. Dessin fait à la chambre claire, le 22 mai 1874, grossi 10 fois.

ARCH, DE ZOOL, EXP. ET GÉX, - $x$, IV. 1873. 
Fig. 2. Larve Âgée de quatre jours el demi, le 26 mai 1874, vue par la lace ventrale et un peu par la droile. Dessin fait à la chambre claire, d'après le vivant, à un grossissement de 200 diamètres.

Fig. 3. Un jeune vivant, âgé de six jours, le 28 mai 1874, vu par le côté dorsal, dessiné à la chambre claire, à un grossissement de 200.

FIg. 4. Un jeune, pêché en mer, vu par le côté dorsal; dessin réduit à un grossissement de 10.

Fig. 5. Le corur, le rein et le bas du bouclier vibratile de la larve représentée sur la figure 2. Dessin fait ì la chambre claitc, grossi environ 400 fois.

FIG. 6. Larve âgée de trois jours, le 12 mai 1874, vue par l'extrémité céphalique et un peu par le côlé droit. Dessin fait à la chambre claire, grossi 200 fois.

Fig. 7. Le système nerveux central d'un exemplaire jeune, un peu plus grand que celui de la figure 4, vu par le còté dorsal, 30 avril 1874. Dessin fait sans chambre claire.

\section{PLANGHE VI.}

Fig. 1. Larve à peine formée de Hyalca labiata, vue par le côté gauche. Dessin fait à la cliambre claire et terminé d'après le vivant, grossi 300 fois. Messine, 11 mai 1874.

Fig. 2. Un paquet d'œul's de la même espèce, dessiné à la chambre claire ef grossi 10 fois.

Fıg. 3. La moitié inférieure du corps de Creseis aciculata, vue par le côté dorsal, dessinée à la chambre claire, d’après le vivant, le 5 mai 1874, et grossie 50 fois.

FIG. 4. La même partie de la même esrèce, exemplaire plus jeune, vu par la face ventrale; même date, mème grossissement; dessiné à la chambre claire.

Fig. 5. Larve de la même espèce, rencontrée ì Arecife (îles Canaries), le 23 janvier 1867. Dessin fait sans chambre claire, réduit à un grossissement de 50 environ.

Fıg. 6. Larve de Styliola subulata, élevée dans mes bocaux, vue par le côté ventral et un peu par la droite. Dessin lait à la chambre claire, d'après le vivant, à un grossissemnnt de $300 ; 11$ mai 1874.

FIg. 7. Larve plus âgée, de la même espèce, prise en mer, vue par le côté dorsal, munie du sac nutritif contractile et d'une plaque dentaire stomacale. Dessin fait à la chambre claire, d'après le vivant, et grossi 200 fois; 30 avril 1874 .

FIG. 8. Le système nerveux central et le tube digestif d'un exemplaire jeune, de la même espèce, dont la coquille a atteint 8 millimètres de long, vus par lc côté dorsal; dessinés à la chambre claire, d’après le vivant, et grossis 150 fois; 17 mai 1874.

\section{PLANGHE VII. - Cleodora lanceolata.}

Fig. 1. Portion d'une chaîne d'œufs pondue depuis dix-heures; chambre claire; grossissement, 5 diamètres.

Fis. 2. Un cul vivant, fraichement pondu, au moment où le nucléus reparait a près la sortie des corpuscules excrétés, dessinć à la chambre claire, ì un grossissement do $200 ; 5$ mai 1874 .

F1G. 3. Le vitellus vivant, an moment oì le nucléus a redisparu pour faire place à deux étoiles moléculaires; origine de la première segmentation. Dessin fait en coupe optique, ì ia chambre claire, el grossi 200 fois. Mème date. 
Fı́. 4. Un œuf pondu depuis quinze heures, 7 mai 1874, vu par le pôle uutritif, dessiné à la elambre claire et grossi 200 fois.

FIG. 5. Un œuf pondu depuis vingt heures, 7 mai 1874, vu par le pồle nutritif, au moment où apparaissent les cils vibratiles. Dessin fait à la chambre claire et grossi 200 fois.

FIG. 6. Une larve vivante à peine foimée, ùgée de vingt-quatre heures, 6 mai 1874, vue par le pôle nulritif ou oral, dessinée ì la eliambre elaire, à uu grossissement de 180 approximativement. L'eetoderme est représenté en coupe optique.

FIG. 7. La même, même àge et mème date, vue par le pòle formatif ou aboral et montrant l'enfoncement préeonclyylien; même grossissement. L'eetoderme est représenté en entier.

Fig. 8. Le bourrelet moteur de la même larve, vu en eoupe uptique, dessiné sans la chambre elaire, à un grossissement de 400 environ.

FıG. 9. Larve, âgée de deux jours, 23 arril 1874 , rue par le còté aboral ou dorsal et par le eòté gauche; dessinée à la chambre elaire, à un grossissement de 200.

FIG. 10. La même larve, même date, vue par le eôté ventral. Les cellules nutritives ou entodermiques commeneent à se diviser, mais la partie centrale du tube digestif ne eommenee pas encore à se différeneier. Chambre claire; grossissement, 200.

PLANGHE VIII, - Cymbulia Peronii.

FIG. 1. Un œuf fraîchement pondu, traité à l'aide acétique au moment où sort te eorpuseule de rebut. Dessiné en coupe optique, à la elsambre claire, et grossi 300 fois. Messine, 21 mai 1874.

FıG. 2. Le vitel'us vivant au moment où le nueléus apparaît au centre, dessiné el coupe optique, à la ehambre claire, à un grossissement de 300 ; même date.

FIg. 3. L'œuf vivant, à l'état de repos, muni de sa vésicule germinative ou nucléus (cellule embryonnaire de première génération). Dessin fait ì la chambre claire et grossi 300 fois. Même date.

Fıg. 4. Le vitellus virant à l'instant où les deux étoiles moléeulaires de la première segmentation apparaissent dans l'intérieur du mucléus; vu obliquement par le pôle formatif et par le côté. Dessin fait à la ehambre elaire, grossi 300 fois. Mlème date.

Fig. 5. Le vitellus an moment où le premier sillon s'est déjà aecentué ì la surface; traité par l'acide acétique, ce qui a fait disparaître le sillon et mis en évidence l'arrangement moléeulaire. Dessin fait à la chambre claire, grossi 300 fois. Même date.

Fig. 6. Le vitellus vivant se segmentant en deux. Dessin montrant les étoiles moléeulaires et le sillon oblique; fait à la chambre claire et grossi 300 fois. Mlême date.

FIG. 7. L'œuf vivant, segmenté en deux, soit le temps de repos du second stade. Dessin fait à la eliambre claire, réduit à un grossissement de 300 ; le 19 avril 1874.

Fig. 8. L'œuf vivant, segmenté en quatre, pendant le temps de repos du troisième stadc. Dessin fait ì la eliambre claire, réduit à un grossissement de 300 . Mème date.

FıG. 9. Le vitellus dépassant déjà le septiènre stade de segmentation; les eellules de septième génération sont en voie dr division. Il est vu par le còté formatif, mais il est mal orienté par suite d'une erreur, la sphérule nutritive céphaliqque se trouvant tournée vers le bas de la figure. Dessin fait is la chambre elaire et réduil at un grossissement de 300 ; le 18 arril 1874 . 
Fig. 10. Le vitellus, un peu plus avancé dans son fractionnement, vu par le côté nutritif, traité par l'acide acétique. La cellule I s'est diviséo sous mes yeux et a donné naissance à la cellule $e c$. Ce dessin, orienté comme le précédent, est porté à un grossissoment de 300 , d'après une chambre clairc; du 22 mai 1871.

Fıg. 11. Portion d'une chaîno d'cufs, fraîchement pondue, dessinée à la chambre claire et grossie 10 fois.

PLANCHE IX. - La mème espèce.

Fig. 12. L'embryon vivant, âgé de deux jours, le 15 mars 1874 , vu par le pôle nutritif. Dessin fait ì la chambre claire et porté au grossissement de $\mathbf{3 0 0}$.

Fig. 13. L'embryon vivant, même aspect, âgé d'un jour deux tiers, le 20 avril 1874. Dessin fait à la chambre claire et réduit à un grossissement de 300 .

FIg. 14. L'embryon vivant, âgé de doux jours deux tiers, lo 20 avril 1874, vu en coupe optique. Dessin fait à la chambre claire et réduit à un grossissoment de 300 .

Fı́. 15. Le même embryon, même aspect, vu dans son entier. Dessiné de même et grossi 300 fois.

FIg. 16. Larve vivante, âgée de trois jours deux tiers (le 21 avril 1874), vue obliquement par le côté gauche et dorsal, montrant l'invaginatien préconchylienue et le rudiment de la coquille. Dessiné de même et réduit à un grossissement de 300 .

Fig. 17. La même larve, même jour, dessinée de même, mais vue par le côté gauche et un peu par la faee ventrale, montrant la partie centrale du tube digestif communiquant déjà avec l’œsophage. Grossie 300 fois.

Fig. 18. Larve vivante, âgée de quatre jours trois quarts, le 22 avril 1874, montrant la masse nerveuse sus-œsophagienue, que j'ai vue se séparer de l'ectoderme dans l'intérieur du voile, et cela chez ce mème oxemplaire, avant de le dessiner. Dessin fait ì la chambre claire et réduit à un grossissement de 300 .

Fig. 19. Larve vivante, âgée de six jours et demi (24 avril 1874), vue par la face ventrale. Combinée d'après deux dessins faits à la chambre claire. Grossissement, 300 .

F1G. 20. L'otocyste et l'otolithe d'une larve âgée de quatre jours et demi, 23 avril 1874. L'otolithe occupe la partie de la capsule qui est en regard avec l'extrémité céphalique de la larve. Dessiné à la chambre claire, à un grossissement de 400.

PLANCHE X. - Clio aurantiaca.

Fig. 1. La ponte toute nouvelle, chambre claire; grossissement, 10.

Fıg. 2. Le vitellus vivant, segmenté en seize ccllules, sixième stade. Il est posé obliquement, de façon à laisser voir son côté formatif, ¿̀ droite et en bas de la figure. Les cellules de sixième génération $1^{\prime}, 2^{\prime}, 3^{\prime}$ et $4^{\prime}$ viennent de se séparer sous mes yeux des cellules 1, 2, 3, 4. La cellule II' est en voie de division et obéit à deux centres d'attraction. Chambre claire, 9 mai 1874 ; grossissement, 200.

Fig. 3. L'embryon vivant, vu en coupe optique pour montrer l'arrangement des cellules de l'entoderme, dessiné à la chambre claire, même date, et réduit à un grossissement de 250 environ.

FIG. 4. Toute jeune larve, au moment où poussent les cils moteurs, vivante et vue par lo côté oral en coupe optique, pour montrer l'arrangement des éléments ento . dermiques, 12 mai 1874. Chambre claire; grossissement, 300.

Fig. 5. La même, même aspect, mème grossissement, vue en entier. Chambre claire.

FIG. 6. Larve au moment où la cavité centrale et l'enfoncement de l'œsophage sont encore séparés, vuo par le còté ventral. Dessin fait à la chambre claire, le 10 mai 1874, et réduit à uı grossissement de 250 . 
Fıg. 7. Larve formée, vue par le còté ventral, dessinée à la cliambre claire d'après le vivant, le 9 mai 1874 , et grossie 200 fois.

Fıg. 8. Le muscle columellaire, bifurqué et muni de deux nucléi, d'une larve du même àge que celle de la figure 6. Il s'insère en haut'au bourrelet moteur et en bas au bourrelet coquillier. Dessiné sans chanbre claire et réduit à un grossissement de 200 environ.

FıG. 9. L'otocyste d'une larve un pen plus âgée que celle de la figure 6, avant l'apparition de l'otolithe, dessiné sans chambre claire.

FIG. 10. Exemplaire probablenent adulte, vu par le còlé dorsal, avec les nageoires, les tentacules et la radule, avec les sacs latéraux entièrement déployés. Dessin fait sans chambre claire et grossi 40 fois. Messine, fin mai 1874.

Les signes et les lettres sont les mêmes pour toutes les figures, à savoir :

I, II, III, IV, les quatre sphérules primitives : I, la sphérule nutritive latérale probablement de droiłe; III, la sphérule nutritive de gauche, sans doute; II, la sphérule nutritive céplalique; $\mathrm{V}$, la sphérule proloplasmique ou inférieure.

$1,2,3,4$, les quatre petites cellules formatives, oll de quatrième génération, provenant de la première division des quatre sphérules primitives; leurs uuméros correspondent à ceux des sphérules qui leur ont donné naissance.

I', II' III', IV', les cellules de cinquième génération, provenant de la seconde division des quatre sphérules primitives.

$1^{\prime}, 2^{\prime}, 3^{\prime}, 4^{\prime}$, les quatre petites cellules de sixième génération, provenant de la division des petites cellules de quatrième génération.

$I^{\prime}, I^{\prime \prime}, I I I^{\prime \prime}, I V^{\prime \prime}$, les quatre cellules de septième génération, provenant de la division des quatre cellules de cinquième génération.

$\mathrm{P}$ f, le pôle formatif; $\mathrm{P} n$, le pôle nutritif; $\mathrm{P}$, le pòle oral ; $\mathrm{Pa}$, le pòle aboral; $\mathrm{Ps}$, le pòle supérieur ou céplılique; $\mathrm{P} i$, le pòle inlíricur.

om, la membrane de l'œuf; on, la partie nutritive du vitellus; of, la partie protoplasmique ou formative du vitellus.

$\gamma n$, les globules nutritifs du vitellıs; $\gamma r$, les granules du protoplasme; $\gamma r n$, les petits globules nutritifs disséminés dans la partie formative du vitellus; $\gamma n$ ', giobules nutritifs qui montrent une struclure intérieure; $\jmath \alpha$, globules adipeux.

v, la vésicule germinative et les nucléi des cellules.

$\alpha$, les centres d'attraction ou centres des étoiles moléculaires.

$\lambda$, la ligne de démarcation entre les territoires de deux étoiles.

$x$, les corpuscules excrétés.

$e c$, l'ectoderme et les cellules du feuillet externe; en, l'entoderme et ses cellules $e n^{\prime}$, cellules différenciées de l'entoderme; ece, l'épaississement postérieur de l'ectoderme; ecc, la partie amincie de l'ectoderme, qui tapisse la coquille; em, le mésoderme.

$q$, la coquille; qi, l'invagination coquillière ou préconchylienne; qir, la rosette superficielle de cellules de l'invagination préconchylienne; qic, le canal de cette invagination; qim, les masses tuberculeuses sécrétées dans son intérieur; qs, la saillie coquillière; $q b$, le bourrelet qui sécrète le bord de la coquille; $q b^{\prime}$, la bande de petites cellules situées au-dessus du bourrelet coquillier; $q l$, la coquille larvaire; qd, la coquille définitive; $q t$, la limite supérieure de la coquille larvaire; qst, les stries de la coquille larvaire.

$c$, les cils; $c c$, les cils de la région céphalique; $c r$, les cirrhes; $c r p$, les cirrhes du pied ; $\mathrm{cm}$; cils moteurs. 
$v$, le voile; $v b$, le bourrelet du bord du voile ou bourrelet moteur; $v c$, les cils moteurs du voile; $v m$, les muscles du voile; $v n$, les petits cils ou cils nourriciers du bourrelet voilicr.

$m$, le manteau; $m c$, la cavité palléale ou branchiale; $m m$, le bord du manteau; $m w$, les cils du mantean; $m b$, le bonclier ou plaque eiliaire du mantean; $m f$, le prolongement dı bord dı manteau du côté droit; $m p$, la paroi qui sépare le corps de la cavité palléale; $m x, \mathrm{l}$ bourrelet ciliaire, c'est-à-dire l'organe des sens situé à l'entrée du la eavité palléale; mch, pigmeni du manteau.

$p$, le pied; pe, épaississement latéral de la région du pied chez l'embryon; $p n$, lobes latéraux da pied ou nageoires; po, l'opereule.

$k$, les sinus; $k n$, le sinus contractile dorsal on de la nuque; $k p$, le sinus contractile du pied; lic, la eavité du eorps; $l i m$, le sinus circulaire du bord du manteau.

$b$, la bouche; $b s$, les saes à eirrhes sur les côtés de la bouche.

$\infty$, l'œsophage; œs, saillie longitudiuale de la paroi supérienre de l'œsophage; ar, la radule.

$s$, l'estomac; sp, replis de l'estomac.

$d$, dents stomacales; $d l$, plaque dentaire provisoire.

$i$, l'intestin.

$a$, l'anus; $a c$, les deux cellules anales.

$\sigma$, les sacs nutritifs; $\sigma_{c}$, le canal excréteur du sac nutritif; $\sigma$, le sac nourricier ven. tral, el $\sigma^{\prime}$, le sac nourricier dorsal.

$f$, le foie.

$h$, le cœur; $h v$, le ventricule; ho, l'oreillette; $h a$, l'aorte.

$r$, le rein; $r c$, le eanal du rein; rop, l'ouverture du canal rénal dans la cavité palléale; roh, l'ouverture du canal rénal dans le péricarde.

$\mu$, les muscles; $\mu c$, les cellules qui donneront naissance aux muscles; $\mu . r$, le muscle rétractcur ou columellaire; $\mu v$, le muscle rétracteur ventral; $\mu . r l$, les faisceaux latéraux du muscle columellaire; ram, le faisceau médian du muscle columellaire; u.n, les museles dn voile attenant à l'invagination nerveuse céphalique; $\mu . p$, les mus cles du pied et des nageoires; $\mu . p s$, les muscles dn pied et des nageoires reliant une paroi à l'autre; u.pl, les museles longitudinaux du pied et des nageoires; u.pt, les muscles transversanx du pied et des nageoires; $u \cdot m$, les muscles du mauteau; $\mu \cdot s$, les fibres circulaires de l'estomac.

$n$, les nerfs; ne, l'épaississement de la région céphalique, qui donne naissance à l'iuvagination nerveuse sus-œsophagienne; $n$, l'invagination nerveuse céphalique ; $n s$, les ganglions sus-œsoplıagiens ou masse nerveuse céplıalique; $n s^{\prime}$, la partie du ganglion céphalique qui se délache ell avant; $n i$, la masse nerveuse sous-œsophagienne; nel, la eommissure latérale, reliant la masse sns-œsophagienne à la masse sous-œsophayienne; ncd, la commissure dorsale, reliant les ganglions céphaliques entre eux; nv, les ganglions viscéraux de l'œsophage; $n n$, les nerfs des nageoires; $n m$, les nerfs du manteau; $n x$, le nerf qui va au bourrelet eiliaire !organe olfactif?). $\omega$, les otocystes; $\omega l$, les ololithes; $\omega c$, la cavité de l'otocyste.

$t$, les tentacules.

Gh, la glande génitale hermaphrodite; Gl, la prartie mâle ou testiculaire de cette glande; Go, la partie femelle ou ovarienne de la glande hermaphrodite.

$\mathrm{G} c$, les glandes cutanées; $\mathrm{Gc}$, les glandes en forme de rosettes de la peau. 
Arcin de Zool Exple et Gen ${ }^{\text {ie }}$
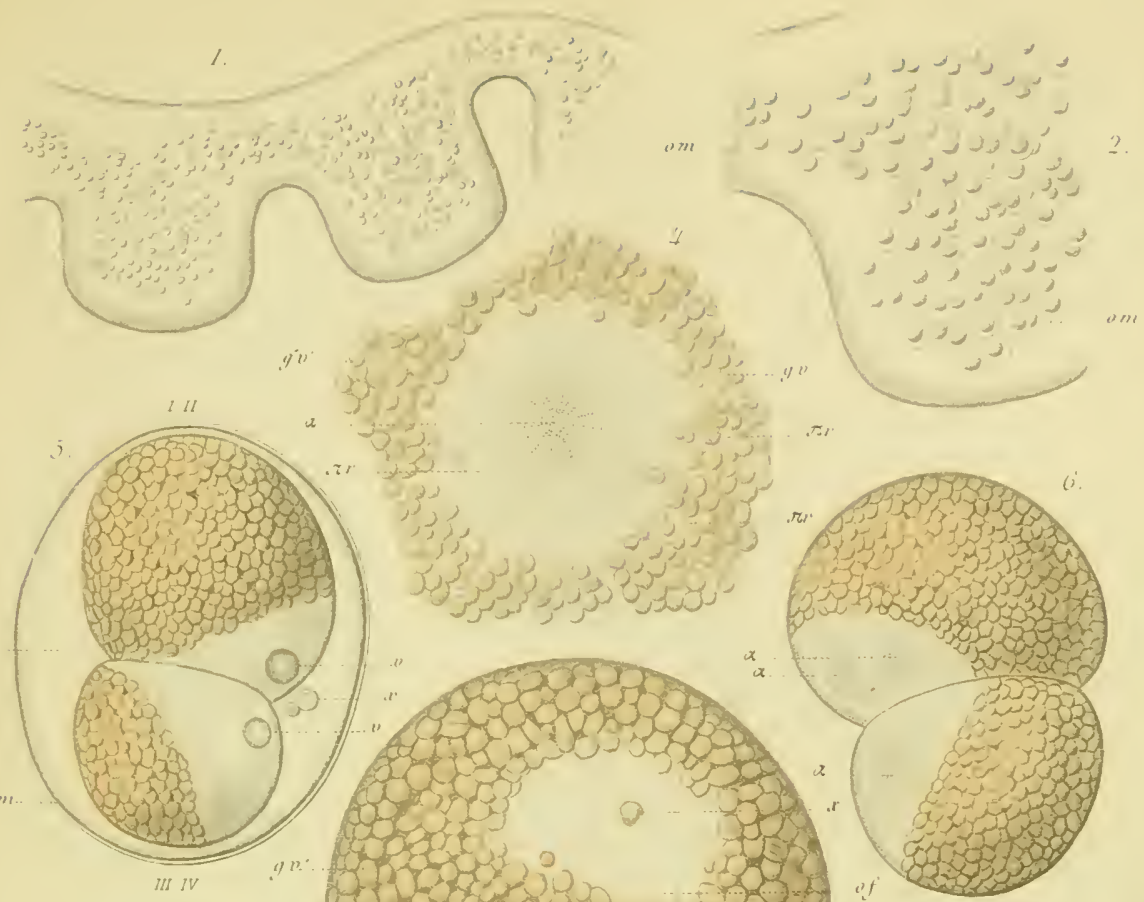

x.

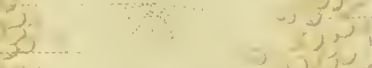

30

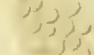

$\int x$ 

A. Lh cie Lool. Exp le et Gente

$\because \quad y=P 1.11$

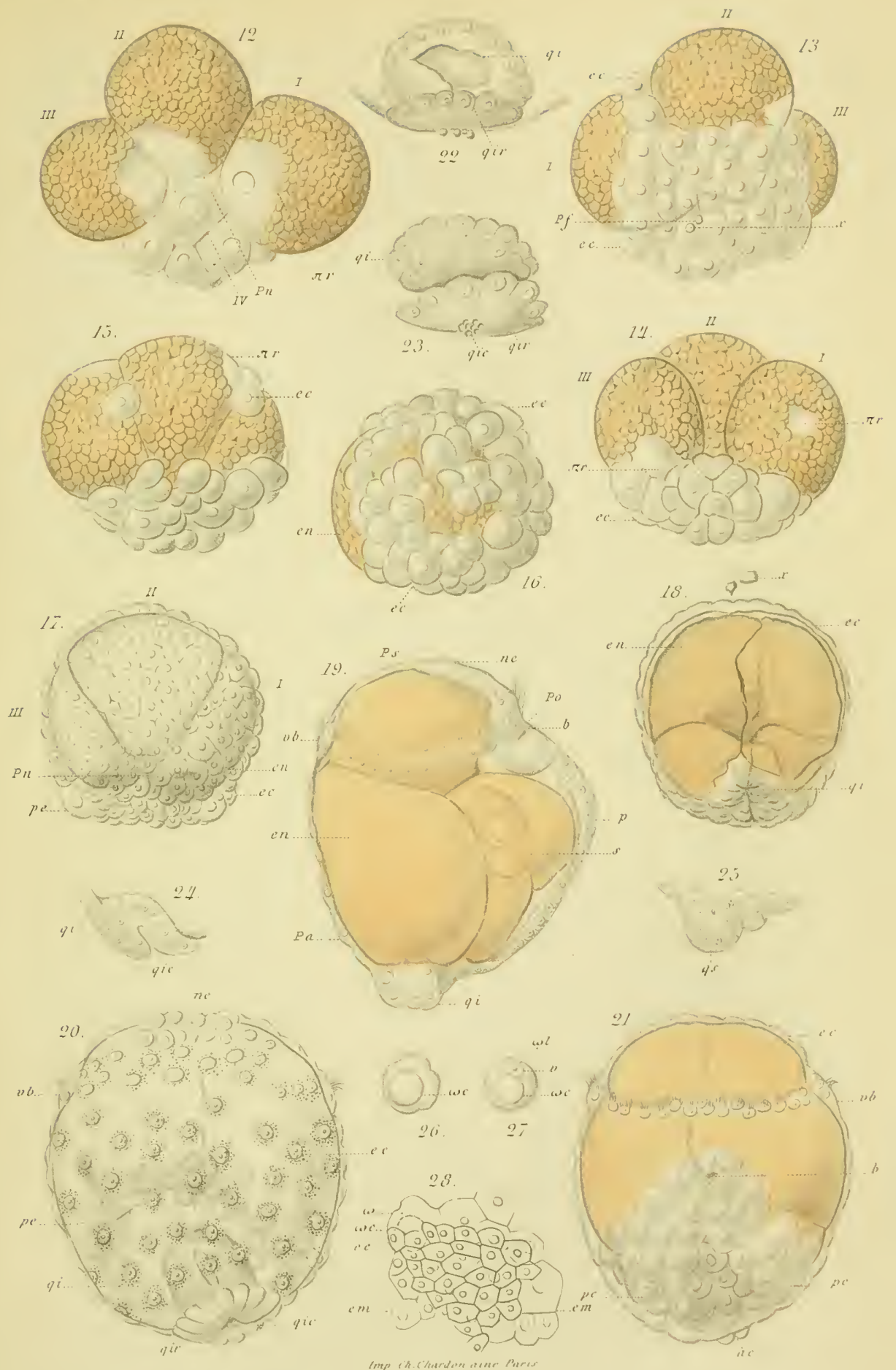

Il till ald nat del

DEVELOPPEMENT DES PTEROPODES I Cavolmia tridentata! 

Arch de 2001 Exple et Gell

Vol IV \& III
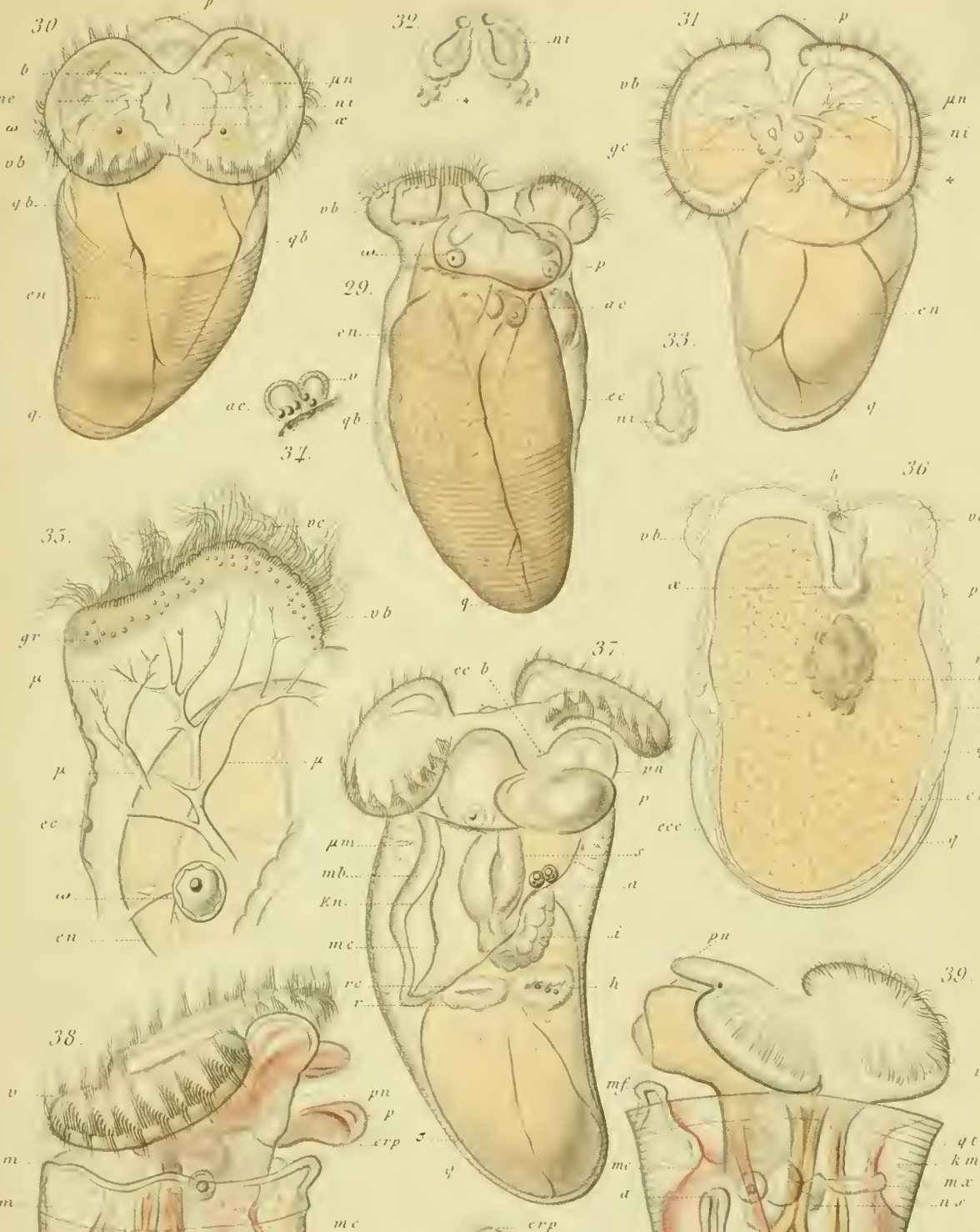

36 

Arch de Zool Exple et Gen ${ }^{\text {le }}$

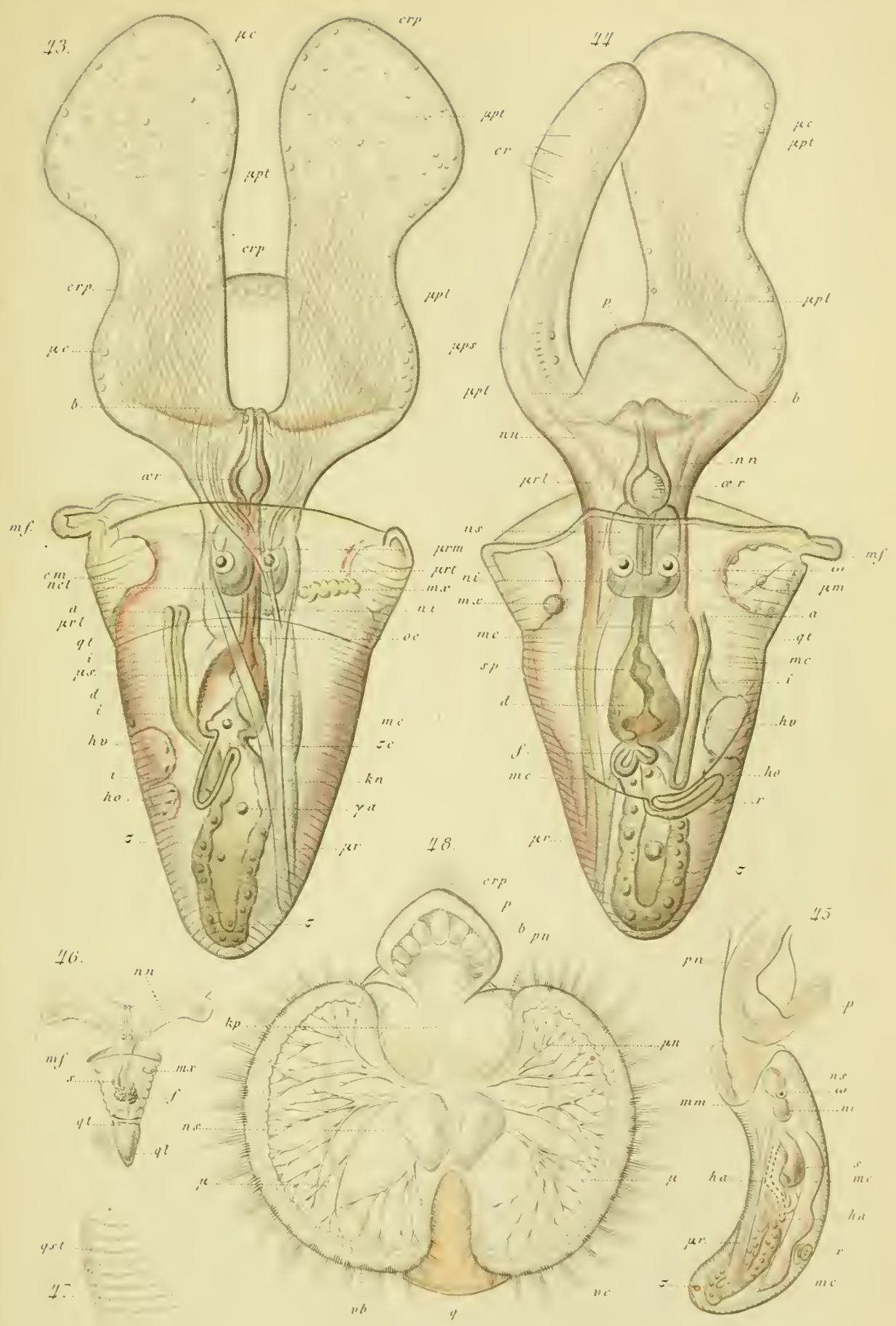

DE:ELOPPEMENT DES PTEROPODES / Cavolma tridentata / 

Arch de Zool. Exppete. Gen ${ }^{1 e}$

Vol IV_Pl V

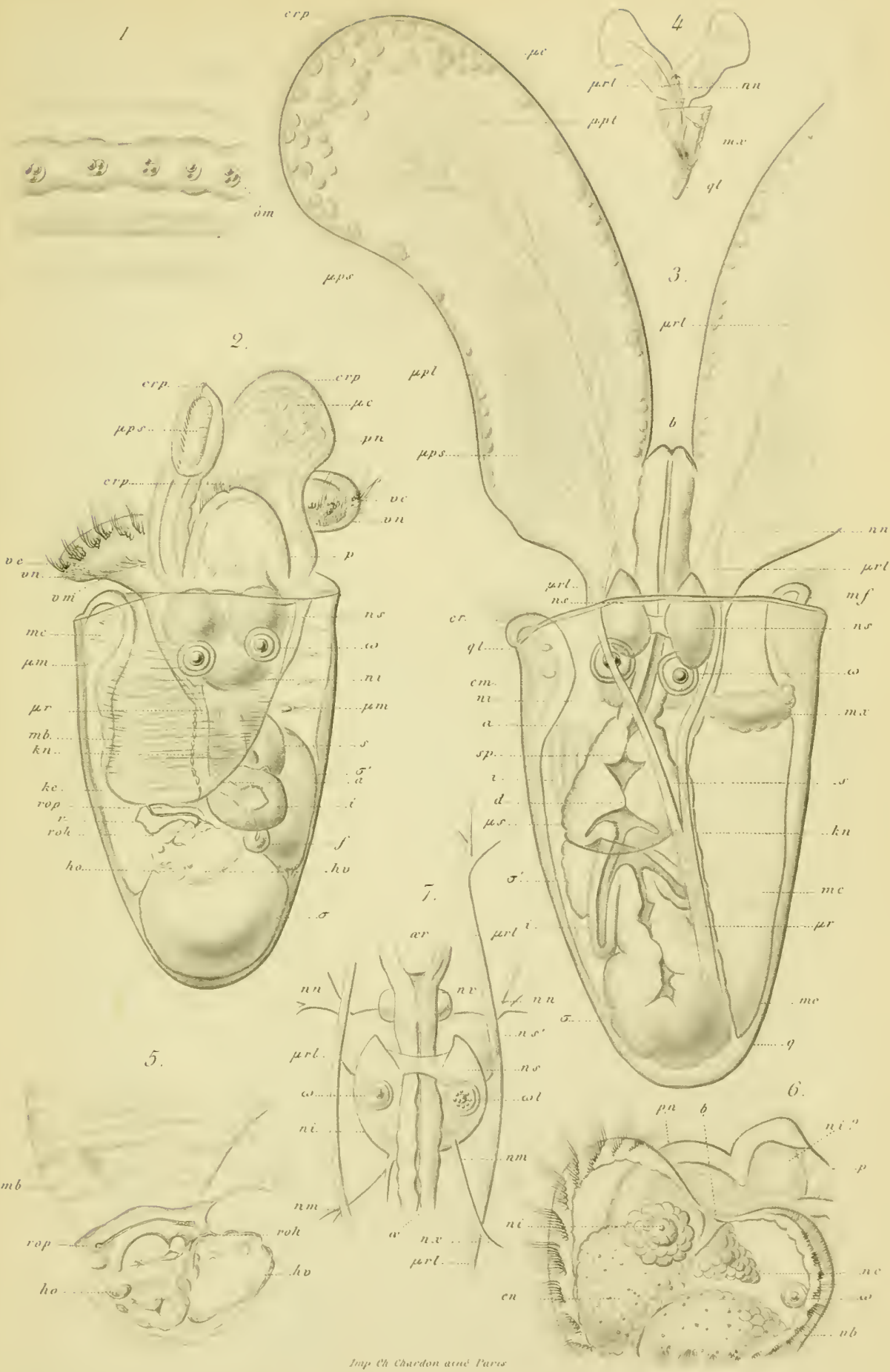

II. Fol ad nal adel

DEVELOPPEMENT DES PTEROPODES / Hyalocylis strata) 

Arch de Z0ol. Exple et Genle

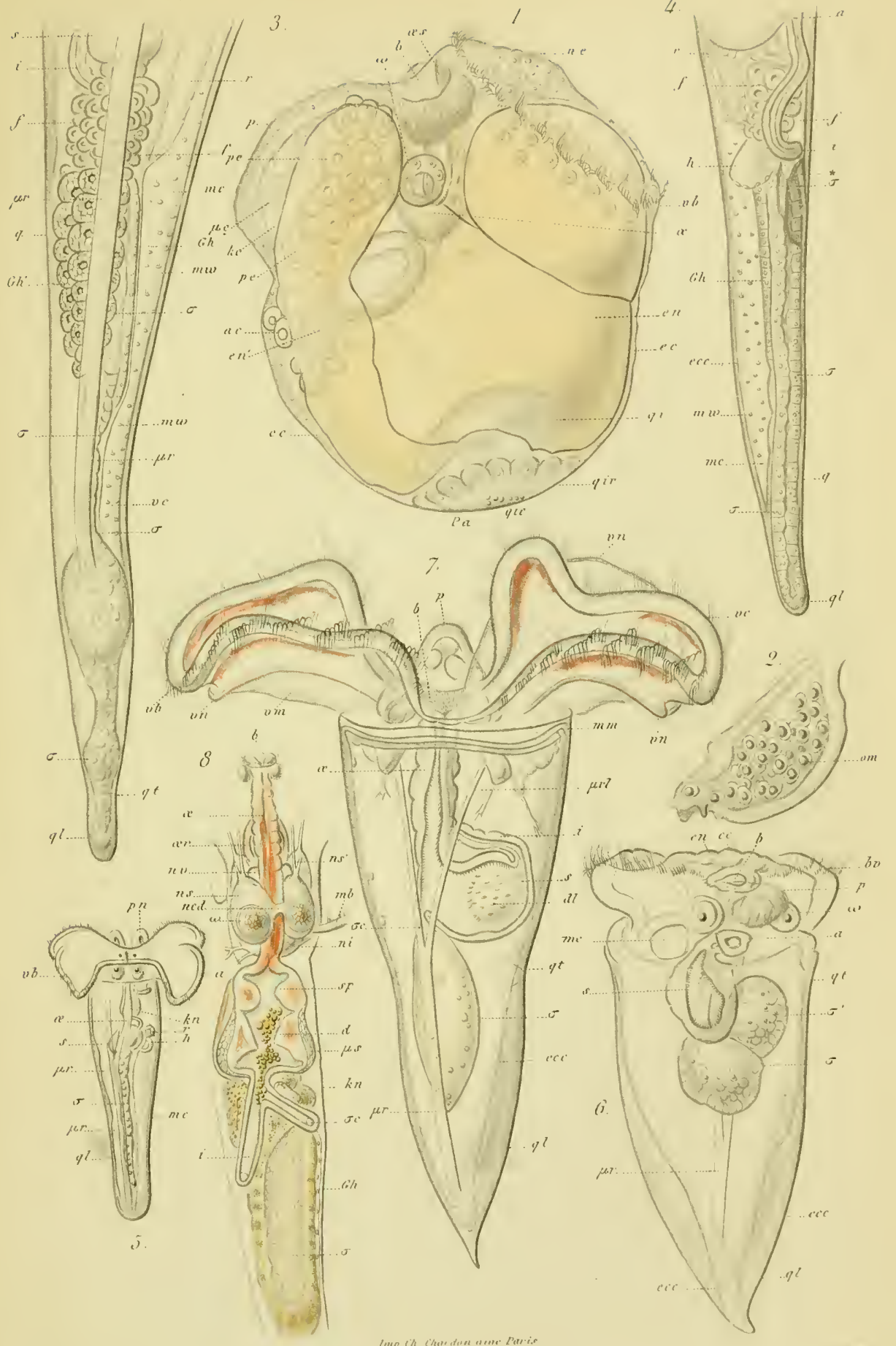





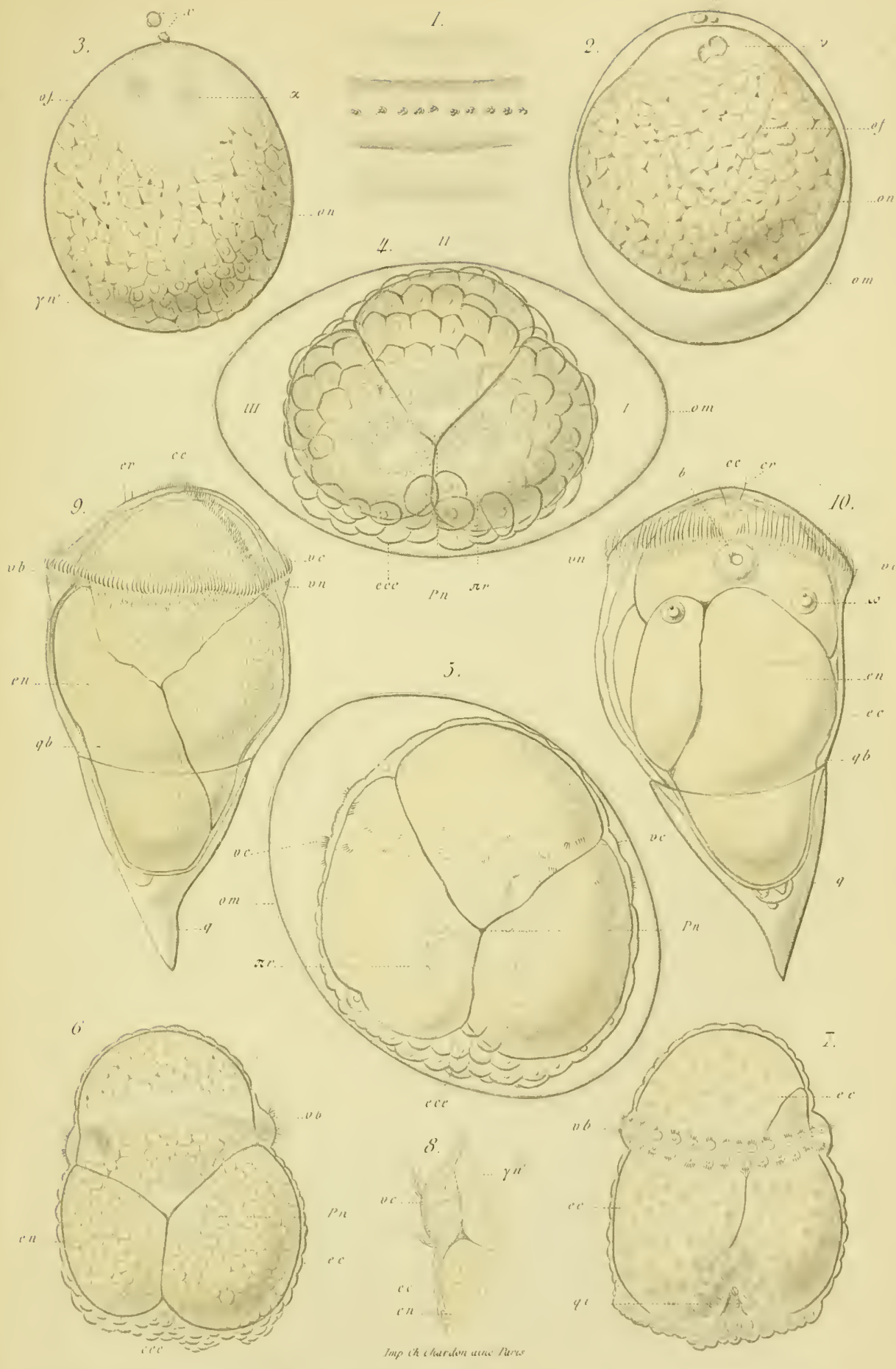

II liblad late dil 


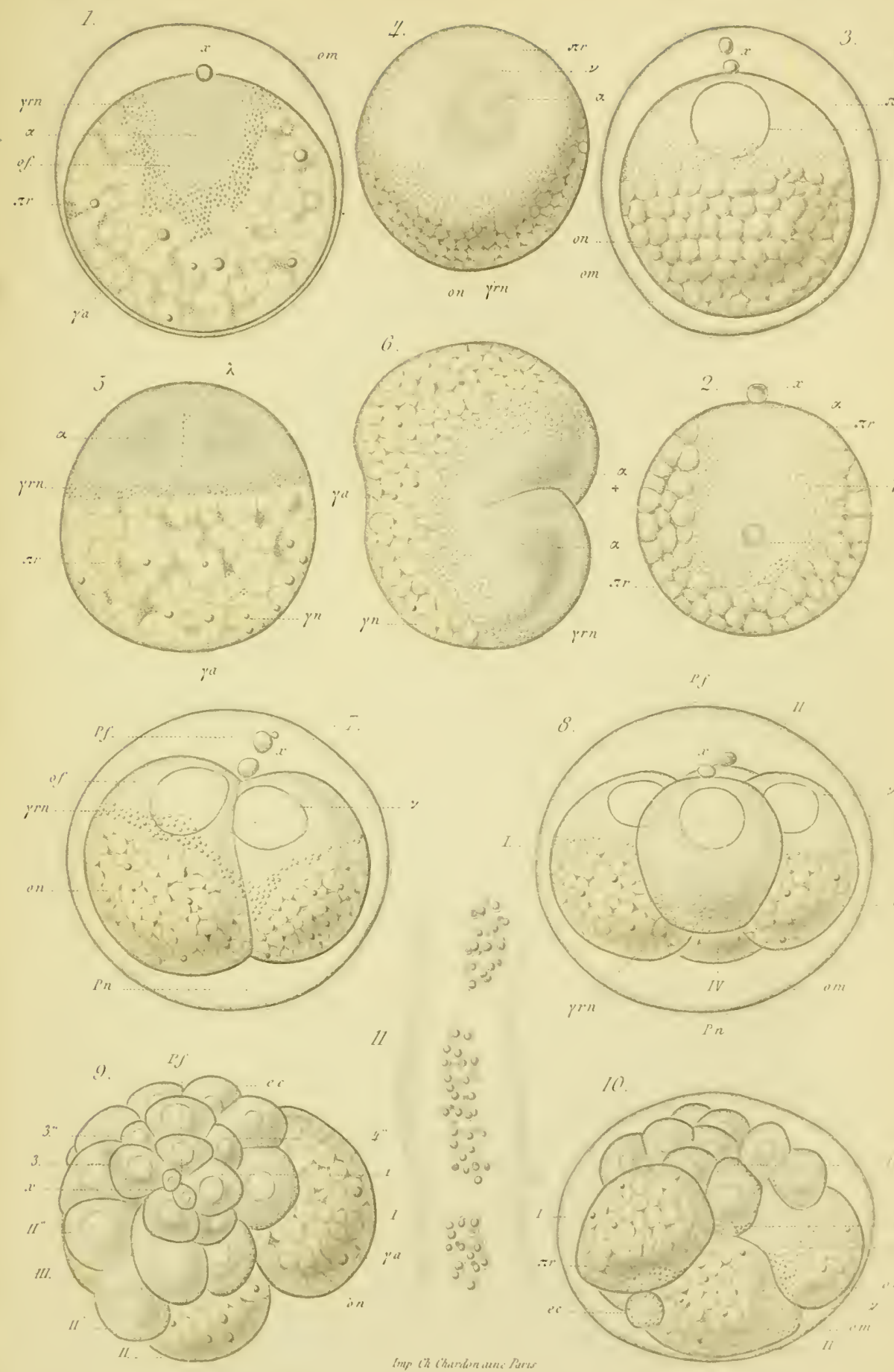

II Fid ad hal del 


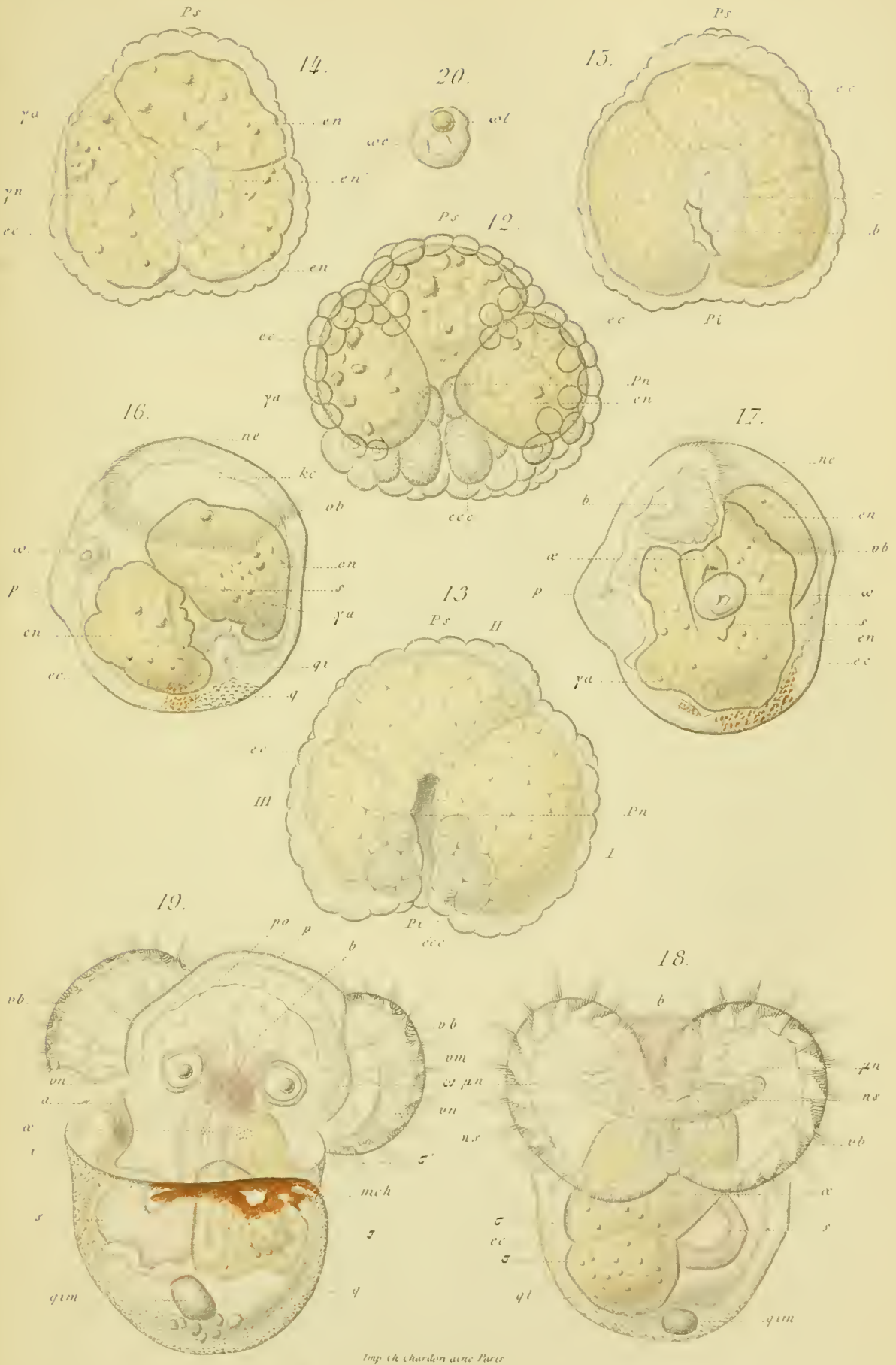


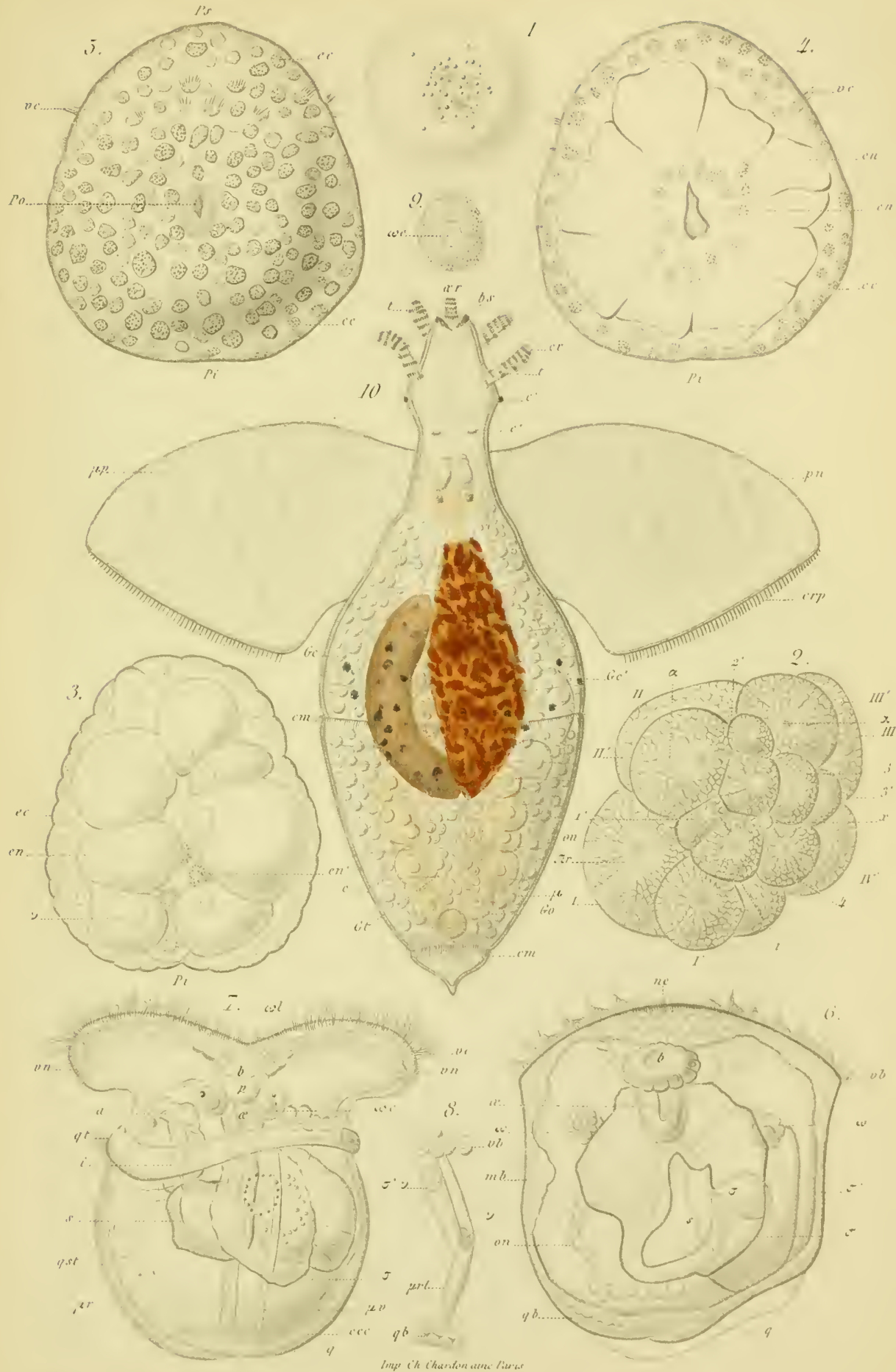

U1. Iid red nate dit

CLIO AURANTIACA 



\section{VII}

\section{ENILE BAUUELOT.}

L'année 1875 s'est tristement ouverte pour les Archives de soologie expérimentale et pour la science frauçaise.

L'un de nos savants les plus distingués, l'un des collaborateurs les plus assidus de ce recneil, Emle Bavdelot, a été enlevé, le 23 février dernier, à peine àgé de fuarante et un aus, dans toute la force et la maturité de sa vive intelligence, laissant inachevés un grand nombre d'importants travaux, n'ayant pas même eu le temps de recueillir les premières récompenses dues à une vie tout entière consacrée à l'étude, et qui déjà avait été féconde en résultats importants pour la zoologie et l'anatomie comparée.

Baudelot (Jules-Emile-Joseph) était ué le 14 mars 1834 à Vendresse, petit village des Ardennes, dans l'arrondissement de Vonziers. Il lit ses études secondaires dans un établissement libre, le collége de la Malgrangc, à Nancy ; vint ensuite à Paris étudier la médecine, et fut reçu docteur en 18ä8; il avait alors vingt-quatre ans.

Compatriote du botaniste Payer, qui tenait en grande estime ses qualités intellectuelles, ses goîts se tournèrent bien vite vers les sciences naturelles. Il songea d'abord à étudier la botanique; mais il ne tarda pas à changer de voie, entra en rapport avec A1. Enile Blanchard et devint bientôt son élève particulier.

Dès son premier unémoire, Baudelot entra dans une voie qu'il a suivic avec une prédilection marquée pendant toute la durée de sa trop courte carrière: l'étude du système nerveux central des poissons. Les Comptes rendus de l'Institut et les Annales des sciences raturelles publièrent, en 1861, ses "Recherches expérimentales sur les fonctions de l'encéphale des poissons "n. Le jeune zoologiste abandonna ensuite momentanément l'étude des animaux vertébrés pour s'occuper des animaux invertébrés.

La constitution intime de l'appareil génital des Mollusques gastéropodes était alors l'objet des vues les plus diverses. II entreprit d'éclairer ce difficile chapitre de l'anatomie comparée et de la physiologie, se retira anx environs de Paris et se mit au travail avec cette ardeur que connaissent bien ious ceux qui l'ont vu à l'œuvre. Dès 1863, ses "Recherches sur l'appareil générateur des Mollusques gastéropodes " avaient pris une inportance suffisante pour constituer une thèse de doctorat ès sciences. La soutenance répondit à la valeur du travail ; elle fut des plus brillantes et, deux ans après, en 1863̆, Baudelot était appelé à remplir, comme clıargé de cours, à la Faculté des seiences de Strasbourg, la place laissée racante par la mort de Lereboullet. Cette même année, l'Académie des sciences couromait un nouveau mémoire de Baudelot, son "Etude sur l'anatomie comparée de l'encéphale des poissons ", qui n’a pas encore été entièrement publicée.

La succession de Lereboullet à Strasbourg était difficile : il s'agissait de retenir autour d'mue chaire qu'il avait occupée non sans éclat peuliunt près de ARCH. DE ZOOL. EXP. FT GEX. - T. 11. $1873^{\circ}$ 
quarante aus les auditeurs qu’avait su réunir l'éminent embryogéniste. Boudelot y réussit. La netteté de son esprit, l'élévation de ses idées, l'amonr enthousiaste qu'il professait pour la science lui rendaient facile ce langage incisif et eoloré, gràce auquel le professeur devient facilement maittre de son auditoire. Il aimait, par uature, les généralisations; il avait cet art de grouper les faits, qui fait naître comme d'elles-mêmes les théories : mais il était trop serupuleusement lonnête pour se donuer le facile plaisir d'étonner son auditoire par la lıardiesse de ces inductions lasardées qui séduisent un moment celui qui écoute, mais n'entrainent même pas la convictiou de celui qui parle. II voulait s'attirer avant tout la confiance de son auditoire; il voulait que les débutants, ì qui il était clıargé d'ouvrir les portes de la science, pussent marcher en toute sécurité ì sa suite; qu’ils se sentissent solides sur le chemin où il les engageait. C'était là une prudence bien méritoire de la part d'une intelligence anssi large, aussi ardente et - nous ponvons ajonter - anssi jeune.

Nais c'est cette prudence, alliée ì un travait soutenu, qui fait les suecès durables, qui assoit les réputatious sur des bases inébranlables. C'est elle qui valut à Baudelot la sympathie et l'ustime du nombreux auditoire qui ne cessa de l'entourer.

En 1868 seulement, après trois ans d'un brillant stage, Baudelot devint titulaire de la chaire de zoologie de la Faculté des sciences de Strasbourg. Il avait, pendant cette première période, publié diverses recherches sur les homologies de plusieurs parties dı squelette ou des membres de certaines espèces de poissons ${ }^{1}$, ainsi que sur les systèmes nerveux et musculaire de ces animaux. Ces pablications eussent été sans donte plus nombreuses, si le professeur u’avait dépensé au savant beaucoup de temps; mais, dès 1869, on s'aperçoit bien vite que le savant a repris le dessus : quatorze notes nu mémoires, portant presque tontes sur l'organisation des poissons, sont pendant cette seule année publiés dans le Bulletin de la Société des sciences naturelles de Strasbourg.

L'année néfaste de 1870 vient malheureusement arrêter ces travaux. Baulelot se souvient qu'il est médecin. Il se fait attacher, comme médecin-major, alı corps d’armée du général Ducrot. Là - bien qu’atteint déjà d’une maladie dı cœur qu’il savait pouvoir lui être fumeste - il se dévoue sans ménagements d’aucune sorte au soulagement de nos blessés.

Arrive la paix ; ̀̀ ce monent le courageux professeur se tronve dépossédé de sa chaire : Strasbourg n'est plus francaise. Que faire? Baudelot revient ì $\mathrm{Pa}-$ ris, non pour solliciter une position nouvelle, mais pour se remettre an travail en attendant des temps meilleurs.

Sur ces entrefaites, M. Duméril, professeur d'ichthyologie et d'erpétologie au Muséum l'histoire naturelle, était mort. Baudelot était, à ce moment, le seul

1 Elude sur le disque céphalique du Rémora ¡ Comptes rendus Acad. des sc., t. LXIV, p. 263, et Ann. sc. nat., 1867). - Note sur le disque ventral du Cycloptère lump (Bull. Soc. sc. nat., Strasbourg, 1868). - Considerations sur les premières vertèbres des ('yprins, des Luches et des Silures (Bull. Soc.nal., Strasbourg, 1868). - Observations sur le rocher dies poissons (ibid.). - Observalions relatives à la piẻce scapulaive des silures (ibid.). 
naturaliste suffisamment prêt pour le remplacer. On le pressa vivement de se mettre sur les rangs. Ses nombreux travaux sur l'organisation des poissons, l'estime dont il jouissait partout, sa situation particulière de professeur spolié par la gnerre rendaient son succès absolument certain; mais il était trop moleste pour ue pas redouter la responsabilité qui incombe ì un professeur du Wuséum. Il préféra se laisser nommer d̀ la Faculté de Nancy aree le titre qu’il avait à Strasbourg. Peut-être la perspective d'un mariage, qu’il contracta peu de temps après à Lmóville, ne fut-elle pas étrangère ì cette détermination.

Quoi qu'il en soit, à Nancy, Baulelot retrouva les succès auxquels l'avait accoutumé son auditoire de Strasbourg. Premier titulaire d'une chaire créée pour lui ${ }^{1}$, privé de lahoratoire, obligé de tont organiscr lui-même, Baurlelot n'en continua pas moins à travailler. Ses belles "Recherches sur la struchure et ie développement des écailles des poissons osseux») datent de cette époque. Il songeait aussi à puhlier eette listoire du système nerrenx des poissous clont quelques chapitres détachés avaient seuls vu le jour, et dont les matérianx, laboriensement accunnlés, étaient tout prêts dans ses cartons.

Il voulait encore écrire un Traité de zoologie générale, destiné ì combler une lacune que tous les $\% 00$ logistes regretteut dans la bibliographie française, et les leçons qu'il rédigea dans les deux dernières années de sa vie et qu'il publia soit dans le Bulletin de la Socièlé des sciences nalurelles de Strasbourg ${ }^{2}$, soit dans la Revue des sciences naturclles de Montpellier ${ }^{3}$, n’étaient autre chose que des essais destinés à venir prendre place dans l'utile et important ouvrage général, dont il mùrissait le plan depuis lolrgtemps. Il en est le même de sa Contribution à l'histoire du système nerveux des Echinodermes".

Ce sont là ses plus récentes publications. Déjit, au mıis d'octohre dernier, ceux de ses amis qui le virent ì Paris, oì il était de passage, furent cruellement attristés en constatant les progrès rapides qu'avait faits une maladie du ceur dont il souffrait depuis longtemps. Ils pressentaient que cette nature l'élite était près de s'éteindre, que cette àme si droite, si sincère, si profonlément honuête, allait les quitter.

Personne néanmoins ne croyait la fin si proche. Quelques-uns le sollicitaient encore de revenir sur son ancienne décision, de se metire snr les rangs pour la chaire d'erpétologie et d'ichthyologie toujours vacante au Muséum, et luimême se décida un moment à poser sa candidature. Iresque en mème temps, !'Académie des sciences, ayant à élire un correspondant dans la section de zoologie, songeait à lui. Dans un rapport lu en comité secret par M. Blanchard, le 22 février, la section le présentait en première ligne. L'élection devait avoir lieu le lundi suivant.

Ialheureusement, depuis le mardi précédent, depuis le 16 février, mne na-

1 A Nancy, comme dans un trop grand nombre de nos facultés de province, un seut frofesseur était chargé de l'enseignement des sciences naturelles. La chaire d'histoire naturelle ne fut dédoublée qu'après la guerre, lors de l'urganisation de l'UniVersité de Nancy.

2 De la méthode comparative en zoologie (Bull. Soc. sc. nat., Strasbourg, 1870).

3 De la zoologie et de ses divisions (Revue sc. nal., septembre 1874).

+ Arch. de zool. expérimentale, I. II, 1872. 
ladie nouvelle était venue frapper Baudelot, qui avait pourtant, ce jour-lù, fait sa leçon conme d'habitude. Huit jours apris, le 23 février, Baudelot expirait sans avoir pu conuaitre l'honneur suprême que l'Académie lui décernait et, comme s'il fallait que le malheur fùt complet, sans avoir pu dire un dernier adieu à sa jeune l'enme, i son enlant, ì peine àgé d’un an, tous deux malades, tous deux à ce moment retenus loin de lui.

Tous ceux qui ont lu les ruvres de Bandelot savent quelle somme éminemment philosophliqute avait son esprit. L'anatomie comparée était son domaine favori. Par une heureuse application de la loi des connexions, par des recherches comparatives très-étendues et surtout très-judicieusement couduites, il avait réussi it résoudre un assez grand nombre de questions controversées relatives an plau de strueture des poissons, aux lomologies de divers or granes de ces animaux. Après cela le système nervenx, siége mystérieux de taut de fonctions encore incomprélıensibles pour nous, avait tenu une grande place dans ses travaux. Il avait fait connaitre la structure si singulière de la chaîne nerveuse d'une remarquable hiruclinće, la Clepsine, avait étudié soigneusement l'histologie du système nerveux des Mollusques acéphales, et avait enlin essayé de déterminer le rôle de différentes parties du système nerveux des insectes.

Le nombre total de ses publications s'élevait à quarante; mais ce n'étaient là que des prémices. Ses travaux les plus importants sont encore en portefeuille, et il est vraiment à souhaiter qu'ils ne soient pas perdus pour la science, qu'une main pieuse les puisse un jour recueillir, mettre en ordre et publier.

\title{
Edmond Perrier,
}

Maître de conférences à l'Ecole normale supérieure, aide-naturaliste_au Muséum.

\section{VIII}

\section{ARBRE GÉNÉALOGIQUE DU RÈGNE ANIMAL,}

\author{
Par M. SeMper.
}

'Traduit, par M. A. Schneider.

Tont le monde connait la belle découverte de $\mathbf{M}$. Semper, relative it l'existence daus les embryons le Plagiostomes d'organes segmentaires homologues à ceux des Anuélides; les Archives ont d'ailleur's publić un résumé de ces recherches. Mais le travail de M. semper renferme, à côté de la partie spéciale diuns laquelle il expose les laits par lui reconuus et dégage leur interprétation, un clapitre de zoologie générale, dans lequel l'auteur nous domne une nouvelle classitication du règne animal sous forme d'uu arbre généalogique, et qui vient ainsi d'autant mieux à point que la Gastran-Théorie succombe chaque jour davantage sous les coups de la critique. La finesse de quelques remarques, la concordance des principaux groupements admis par 
M. Semper a vec ceux qu'a tonjours défendus l'école française; la nouveauté, enfin, de quelques aperçus, nous ont donné lieu de peuser que les lecteurs des Archices auraient plaisir ì pareourir ce chapitre, et, pour ne rien enlever à leur satisfaction, ıous avons préféré traduire ì analyser.

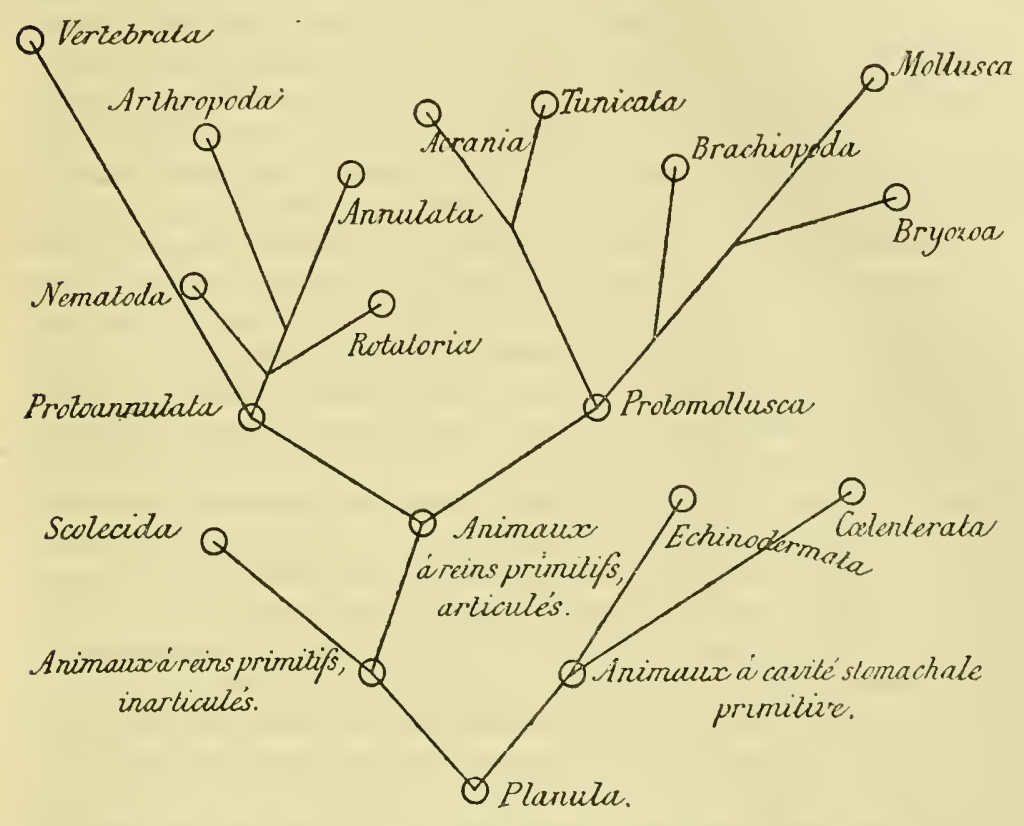

Nouvel arbre généalogique monophỵlètique du rẻgne animal, londé sur la thénrie des reins primitifs et sur l'ensemble de l'organisalion des animaux.

Quelque peu enclin que je sois, d'ailleurs, à étendre trop loin les conséquences des faits précédents et à me risquer aussi en l'établissement d’arbres généalogiques hypothétiques du règne animal, je n’en veux pas moins cette fois, pour différents motifs, satisfaire à la mode dominante. J'ili en partio basé l'arbre généalogique qui suil sur les reins primitifs ou orgaus segrmentuires, et en partie je me suis appuyé sur la conviction que le phylum des Vers, qui revient eomme un spectre bruyant dans tous les traités, u’a par le fait aucun droit à l'existence. Voici done ici d’abord l’arbre généalogique en question; je le ferai suivre de quelques remarques d'ordre plus spécial d'abord et ensuite d’importance plus générale.

En ce qui concerne les rapjorts des Protozoaires aux Métazoaires, la lumière est suffisamment faite; je les laisse donc en dehors de inon cadre, les changements apportés ne devaut les atteindre en rien d'essentiel. Tout aussi peu discuterai-je iei la question de sivoir si l'on doit considérer comme forme ancestrate des Métazoaires la l'lamula ou la Moruln; pour moi, éest la Pla. mula que je préfẻre. D'elle, ì mou avis, sont issus deux trones principaux : les 
animaux à cavité stomachale primitive (Urmagenthicre) et les unimaux à reins primitifs (Irnierenthiere).

Le type des premiers est le Gastron. De son estomac primitif, en simple culde-sac, sont provenus, l'un côté, le système des cananx des Cœlentérés; de l'autre, le système des vaisseaux ambulacraires et la cavité du corps des Echinodermes. En te yui touche le système colentérique des Caelentérés, j’ai déjì plusieurs fois exprimé inon opinion, et je me trouve sous ce rapport en aceord sur les points essentiels avec Hackel; mais e'est une manière de voir tout a fail opposée ì la mienne que celle qui fait des Echinorlermes pour ce naturaliste une colonie de Vers articulés. Une objeetion antérieurement déja présentée par moi, et il liquelle il semble qu Hieckel n'ait pas le moins du monde songé, doit ètre iei répétée, à savoir' :que l'Holotlnurie ne nait pas de cing antimères distincts, comme l'exigerait l'hypothèse. Co ì quoi j'ajoute que la première éhauche de l’Annélide se constitue par deux moitiés latérales de la bandelette embryonnaire venant se souler l'une à l'autre sur les deux lignes médianes, et par conséquent dans un plan vertical, tandis qu au contraire lit prenière ébauche d'une des parties radiaires d'un Echinoderme résulte de la sondure latérale d'une moitié ventrale et d'une moitié dor'sale. La similitude dans la gemmation de nouveaux membres chez les Vers et ehez les Echinodermes n'implique aucune ilentité đlans la nature du phénomène. J'ajouterai encore que, suivant les recherches de Mecznikow, la eavité dı corps des Echinodermes résulte de la coalescence des denx corps le Müller, de façon qu’au cas oì ce résultat serait conlirmé, les Echinodermes seraient privés d'une cavité générale proprement dite (cavité pleuro-péritonéale). En tous cas, ce qui est értain, c'est que cet appareil si éminemment caractéristique du type échinoderme, le système des vaisseaux aquifères, provient de l'intestin primitif de la larve. Il n'y a de difficulté que pour les Crinö̈des, pour lesquels le même rapport loit être nié de la façon la plus absolue. La forme de leur larve rappelle singulièrement la forme polype des Cuelentérés et me parait dénoter avece ces derniers des rapports pour le moins aussi étroits que ceux qui semblent se présenter avec les Oursins da!ı la formation des parties squeleltiques, notamment des Cystiltes et les Blastoïles. Ce deruier point. saus doute, devrait fiare l'objet de nouvelles recherches. L'idée d'une étroite parenté d'origine entre les Annélides et les Echinodermes - mais non de la facon dont Hieckel la conçoit - semble avoir en sa laveur un parallèle entre certains urganes des Sipunculides et des Holohlhuries, que j’ai le premier mis en lumière. Gegenbaur l’a reproduit plus tard, ì la vérité saus me nommer. Chezles vrais Sipunculides existent des entonnoirs vihratiles que, même anjourd'hui, après la communication le kay Lankester il ee sujet, je connais mieux que pits un pour ce qui a trait à leur structure et à leur apparition; ils ne sont pas creux, ne débouchent nullement dans un canal et ne sont en aucume façon comprarables aux organes segmentaires des Annélides. Dans un antre groupe (Thalussema, Boncllia) on trouve, à l'extrémité de l'intestin, deux utricules avee entomnoirs vibratiles s'ouvrant dans la cavité dn corps. Ce sont la peut-être des organes segmentaires transformús, encore bien qüindépendamment d'eux il y ait anssi de ces organes segmentaires typinques, et même dans les Thalassemil jusrun id trois paires. Chez les Synaptes, il y a des entonnoirs 
vibratiles, semblables à ceux des Phascalosomu, semblablement répartis; ici aussi ils ne sont en communication, je duis le répéter, de la façon la plus absolue, ni avec quelque vaisseau que ce soit, ni avec aucune espèce de cavité. Chez les Holothuries, comme chez les Thalıssema, la communication avee un canal manque également; mais, comme les Thalassema, on trouve à l'extrémité de l'intestiu deux ou trois cœcuns qui offrent une grande ressemblance avec ceux de. la Boliellie. Haintenant, je dois it la vérité déclarer que, dans ce parallèle, je ne puis voir qu'un évémement fortuit. D’ailleurs, en voulût-on faire l'indice d’une parenté généalogique, que la conception de l'Echinoderme-Ter d'Hackel en recerrait la plus grave atteinte; car, dans aucun autre groupe d'Echinodermes n'existent, réalisés d’une façon typique, des organes qu'on puisse comparer aux organes segmentaires des Vers, non pas même chez les Astéries, nnalgré qu’elles doivent être la forme ancestrale, de laquelle les Hololhuries ne sont dérivées que par réduction.

Le second grand trone est celui des animanx inarticulés à reins.primitifs, que je earactérise essentiellement par la présence d'organes segmentaires dérivant du füullet moyen, organes qui sont demenrés recounaissables aussi bien dans les représentants inarticulés que dans les représentants articulés de ce phylum. La forme la plus simple du type inarticulé nuts est conservée par les Vers plats (Scolécides), dont les larves mêmes montrent quelquefois aussi l'entonnoir vibratile de i'organe segmentaire librement ouvert dans la cavité du corps. Généralement parlant, ces organes segmentaires sont conformés d'une façon toute particulière et typique pour la classe entière. A raisou de ce que plusieurs organes manifestent me tendance a l'articulation et de ce qu'il existe un système circulatoire, je sépare les Némertiens en tant que Rhynchelminthes et je les rattache aux Annélides proprement dites. Quant a un rapport entre ces dernières et les colonies de Vers rubanés, je ue puis le reconnaitre, nonobstant l'avis de Gegenbaur. La formation des articles dans les Cestoüdes procède de façon tout autre quue la segmentation des segments primitils, dans la bandelette embryonnaire de l'Euaxes par exemple. Chez celuici, c’est le segment le plus reculé qui est toujours le plus jeune, lans les Cestoïdes, il est sans exception le plus âgé.

Par la segmentation de l'animal simple à reins primitifs sont provenus les aniınaux articulés à ruins primitifs. Mais ici se rencontrent d'importantes lacunes dans nos connaissances, et elles ne peuvent encore présentement être comblées. De ce fait que, dans les Vertébrés, dcraniens, certains Tuniciers, ct vraisemb]ablement aussi dans les Annélides, il y a un cordon cellulaire qui se constitue en une chorle dorsale, arant même que la segmentation en protovertèbres on en segments primitifs se snit manilestée, on peut conclure que la forme ancestrale inarticulée des animanx précités, c'est-à-dire l’individı qui s'est transformé en le premier animal articulé à reins primitifs, a dì, lui anssi, posséder une chorde dorsale. Quant in un individuremplissant ces conditions, il est de fait eucore inconun aujourl'hui. Comme réalisant le mieux le type de l'animal articule à reins primitifs, éest le Bulunoglossus que je considère. Quelquefuis encore rapporté aux Annélides, il laisse, d’autre part, reconnaitre certains rappnrts avec les Némertiens, aussi bien qu'avec le mer-




que par des recherches très-précises sur des points déterminés. D’après ce qui précède, on aurait comme caractéristiques des animanx articulés à reins primitifs, en premier lieu, l'élément rein primitif; en second lieu, les segments primitifs ; en troisième, la cage branchiale, laquelle est chez le Balanoglossus exactement constitnée comme chez l'Amphioxus.

Ce tronc s'est ensuite subdivisé en les deux rameaux des Protoannélés ef des Protomollusques. Par le premier terme, je désigne la forme ancestrale hypothétiquement admise de tous les animaux chez lesquels la segmentation en segments primitifs, sous les modifications partielles que ceux-ci ont pu éprouver, s'est conservée avec une suffisante netteté, aussi bien par conséquent que la répétition par segment des reins primitifs; quant à la cage branchiale, au contraire, elle a continué ì se développer dans le groupe des Vertébrés, tandis que dans celui des Annélides, elle a complétement (?) disparu. A ces dernières se rattachent par d'étroits rapports les Arthropodes, cliez lesquels il est possible que le système trachéen doive être considéré comme le terme homologue très-particulièrement modifié des organes segmentaires, qui sont déjì, chez les Annélides et Vertébrés, passibles de si diverses transformations. J'appuie ce soupcon sur le mode d'origine des trachées dans l'abeille, d'après les travaux de Kowalesky; les rapports de position de ces organes an reste de l'economie sont essentiellement les mêmes que ceux des organes segmentaires dans les Aunélides. Des recherches minutieuses sur le mode de formation de la portion glandulaire des trachées sont encore nécessaires toutefois avant qu'on puisse exprimer sur ce sujet une opinion réfléchic. Les Nématoüles dénotent, sous le rapport de lil musculature et par les lignes latérales, une étroite parenté avec les Annélides; mais, ì raison de la conformation spéciale des canaux renfermés dans les lignes latérales, ì raison de ce que ces Vers n’ont qu'un développement abrégé, que la perte est complète chez eux de la segmentation et des organes segmentaires; ce sont certainement aussi des êtres profondément différenciés. Les Rotateurs aussi forment un groupe à pari, et il est fort discutable si on doit le rattacher à ce rameau des Protoannelés. Je les y ai rapportés ici avec doute, parce que leurs organes segmentaires, dont il y a plusieurs paires, montrent des entomnoirs ouverts dans la cavité du corps.

Le second rameau, celui des Protomoliusques, a, au contraire, complétement perdu la division primaire en segments primitifs et les organes segmentaires, pendant que la cage branchiale est restée en partie sous sa forme typique et en partie, suivant une autre direction, s'est transformée en branchies symétriquement disposées. Sur les rapportí de parenté des véritables Mollusques (Céphalopodes, Céphalophores et Lamellibranches) aux Brachiopudes, l'accord est ì peu près unanime; il n'y a que Morse qui soutienue la thèse que les Brachiopodes sout de véritables Vers, se fondant en cela essentiellement sur le facies de Ver qu’offrent leurs larves et sur la présence d'organes segmentaires en la forme typique de ces parties. Que les saes brunàtres des íingula, Terebralula, etc., pris d'abord pour des cœurs, mais, en fait, remplissant les ròles de spermoductes ou d'oviductes, correspondent aux canaux en lacet des Annélides, e’est ce que Gegeubaur avait déjì reconnu; mais, en même temps, ce zoologiste insistait sur ce point. 1ue les reins dur reste 
des Mollusques étaieut exactement constitués et disposés suivant le plan de ces mêmes organes. C'est aussi ee que mes propres reclierclies me permettent de confirmer, et jajonterai que les entonnoirs, par la façon dont ils s'ouvrent dans le péricarde, rappellent, dans le Tridacna, les pavillons vibratiles des Annélides d’une façon bien plus prononcée qu'en aucun antre bivalve. Pourtant Gegenbaur n'en a pas pris occasion, et sans aucun doute avec raison, pour placer les Mollusques, non plus que les Brachioporles, parmi les Vers ; car, encore bien que les organes segmentaires déposent d'une facon très-nette en faveur d'une forme ancestrale commune aux deux groupes, il n'en subsiste pas moins, d'un autre côté, que l'organisation de ces grroupes, dans son ensemble, a suivi ultérieurement une direction si divergente que cette divergence aussi doit trouver son expression dans le systime. Quant an facies Ver des larves, il n'y faut attacher aucune importance, puisque la même forme se rencontre aussi chez de vrais Mollusques, dans les Planariés, Echinodermes et heaucoup de Cœlentérés; c'est précisément la Planula ou Gastrula (comme on veut) bilatérale. En ce qui concerne l'absence de tonte segmentation du corps de l'animal adulte, on pourrait sans doute exprimer l'opinion que les Bracliopodes sont issus directement des animaux inarticulés à reins primitıls, tels que les Scolécides; mais je crois pourtant trouver, sous beaucoup de lapports, l'indice que lis Mollusques ne sont devenus que secondairement inarticulés par une sorte de métamarphose rétrograde. Voici brièvement les motifs de cette opinion: dans les Céphalophores nous trouvons le genre Chiton, signalé par une larve qui rappelle d'une façon peu commune celle d'une Aunélide; à cela s'ajoute la division en segments de la région dorsale, exprimée anssi sur la coquille, et le mode de développement des poils qui en garnissent les bords, semblable ì celui des soies des Annélides. Parmi les Opistobranches, les trones biliaires qui se rendent aux appendices dorsaux se répètent souvent par paire arec une extrême réguiarité, et même, dinns les parties génitales, le Tergipes laisse percer une tendance à la répétition. Le Pueumodermon offre, comme beaucoup d'Annélides, une larve pourvue de plusieurs anneaux de cils (trois); dans le Dentale, le nombre en est plus grand encore (six). Parmi les Brachiopodes, les Thécilies et Térébratulines ont de leur côté une larve extrêmement semblable aussi à celle d'une Anuélide. Les soies de tous̉ les Brachiopodes se développent exactement comme celles des Annélides. Sans doute, ces rapports ne sont pas cumplétement décisifs, et il denteure toujours possibleque les recherches ultérieures enlèvent toute importance ì ces indices de l'existence antérieure d'une forme ancestrale articulée pour les Mollusques. et qu'elles dévoilent et prouvent les véritables rapports de ces êtres avec les Scolécides.

Jusqu’à quel point les Bryozoaires sout ici à leur véritable place, c'est ce que de nonvelles recherehes apprendront. On en a fait récemment des Vers; mais, dans le fait, il est difticile de trouver un rapport queleonque entre eux et les autres animaux rapportés ì la classe des Vers. Nulle apparence d'un système cirenlatoire; pas d'indice d'articulation dans le corps; absence complète de tout système rénal; rien non plıs d'une ligne latérale. Il n'y a de quelque peu favorable à la comparaison que la couronne tentaculaire, puisque dans le Phoronis, qu’il faut décidément rapporter aux Géphyrieus, on en 
trouve une toute semblable. Mais cette couromne tentaculaire aecuse en beaucoup de cas une division manileste en deux moitiés symétriques séparées par le plan vertical mené par la bouche, l'intestin, l'anus et le ganglion nerveux ; en moitiés droite et giuche par conséquent. Les larves (Cyphonautes) de beaucoup de Bryozoaires ressemblent aussi beaucoup par leurs cogrilles et l'intestin a celles des Mollusques. Dins le curieux Rhabdopleura d'Allman, si étumnamment laissé daus l'oubli, on trouve chez le jeune être, aussi bien que sur les Blastuzoïtes, deux valves d'abord assez grosses, distinctes en droite et gatuche et unfourant le corps. Elles teviennent ensuite graduellement rudiuentaires, mais en demeurant toujours visibles. Je vois dans ces faits un point l'appui suffisant pour placer provisoirement ces animanx dans le voisinage des véritibles Mollusques, en tant que classe dill'érenciée d'une facon tonte spéciale d'aillenrs et, par conséquent, indépendante.

Ce que je viens de dire pour les Bryozoaires, je le répeterai presque exactement pour les Tuniciers. La ressemblance de leurs larves avec celles des Trématodes, alors mêne qu’elle serait plus qn'extéricure, ne dénoterait de rapports yu’ivec les Scolécides. La formation d'une valve ventrale et d'une valve dorsale dans le Chevrenlius et les prolongenents vasculiformes de la cavité du corps dans le mantean, dénotent de très-étroits rapports avec les Brachiopodes. Il est vrai que, l'autre part, les reins prinitifs leur font complétement défaut, et que l'organe qu'on appelle rrin dans les Ascidies n'a certainement rien à faire avec les organes segmentaires. Sous ce rapport, les Tumiciers s'éloignent lone aussi beaucoup des véritables Nollusques et des Brachioporles. Mais, l'un autre côté pourtant, lesur cage branchiale, pour moi comme pour van Beneden et von Baer, trahit des rapports manifestes avec les Lamellibranches notamment, et les rapproche plus encore sur ce point de l'Amphioxus, que je crois devoir, en tant que représentant des Acrania d'llæckel, éloigner complétement du voisinage des Vertébrés.

Rippeler ici les raisons yni militent eu faveur l'une étroite parenté de l'Amphio rus et des Ascidies serait chose superflue; chacun les sait de reste. Mais je dois, an contraire, expliquer pourquoi je no puis considérer l'Amphioxus comme un Vertébré, et non pats mème comme un itre proche parent les Annélides. Que le morle d’orisgine du eanal rachidien ne décide rien, c’est ce que j’ai déji expliquué. Dans la Truite, ce canal ne nait pas comme daus l'Amphioxus et les antres Vertélorés, mais exactement de la même façon que la chaine abdominale des Amnélides. Les premiers débuts de la chorde dans l'Amphioxus concordent avec ce pui se passe dans les antres poissons; mais après, ainsi que nous lont appris les belles recherches de Kossmann, elle continue son évolution d'une façon ì elle propre. Comme maintenant il demenre ancore possible que la chorde les Vers dont il a été question plus liaut. soit originellement identique dans si structure avee celle des Vertébrés et de I'Amphioxus, il ne faut provisoirement attacher aucune importanceparticulière an lait de sal présence dans ces derniers, et d’antant moins yu'on designe sous le nom de chorde dans les Ascidies un corton cellulaire, gui ne coinciele aree la chorde des Vertébrés qua ju point de vue de sa position par rapport aux organes enviromuants dans les jremiers états de la larve, mais mullement atu point de vue de sa structure primitive or la struclure est le seul raractione 
décisif. Tous les autres iapports dont on a voulı arguer dans le même sens ne prouvent rien. La partition de la musculature en segments se rencontre aussi dans les Annélides, où les septil (entre tous dans les Polygordius) répondent complétement aux eloisons intermusculaires des Puissons, dirigés toutefois un peu dilféremment; et encore nème daus l'Ammocœte feur direction devient-elle sulfisamment concordante avee ce qui a lieu dans les Vers. Le canal entanć, découvert par Rathké, venant s'onvir près du pore ablominal, est, tout aussi peu yue la ligne latérale des Nématoïles, à considérer comme l'homo!ogne des organes segmentidires. Si la cage branchiale, enlin, eoncorde dans l'Amphioxus et les Vertébrés, on la iroure aussi semblablenent constituée dans les Ascidies et le Balanoglossus; elle ne prouve donc rien.

Or, d'une importance décisive est la somme des caractères qui lémoignent contre la parenté en question. Daus l'Amphiorus, le système des reins primitifs manque entièrement; pour tous les antres Vortébrés. il est éminemment caractéristique; la division de l'axe en segments n'est en rien exprimée sur les éléments du squelette, et dans les Pétromyzontes mêmes, elle ne lait pas tout à fait défaut; nulle distinction entre un cerveau et une moelle, organes profondément séparés l'un de l'autre dans le reste des Vertébrés; tous les trones vaseulaires pulsatils ahsolument comne dans les Vers; Jeur ulistribution même plus conforme à ce qu'elle est dans les Invertébrés; les organes des sens sont tout nutres que dans les Vertébrés; la larve est une Gastrula libre-nageuse, qui n’apparait en aucun Vertébré; l'organisation des organes reproducteurs, enfin, diffère tont aussi complétement de celle des Vèrtébrés. Pourtant j’attacherais peu de poids ì toutes ces différences, si par leur mode de développement la cage branchiale, le système nerveux et la chorde des Vertébrés étaient dans le fait aussi caractéristiques de ce groupe qu’on l’a admis jusqu'à ce jour; mainteuant, an contraire, on le sait, toutes ces particularités se rencontrent également plus ou moins concordantes en nombre d'Invertébrés, et le plus inportaut de ces earactères, la formation d'un tube médullaire, ne se réalise pas même dans tous les Vertébrés de la mème façon; toutes les anomalies citées plus haut de l'Amphioxus vis-à-vis des Vertébrés acquièrent done maintenant une valeur qu’on ne leur attribuait pas jusqu’à ce jour, savoir : celle de earactères différentiels qui doivent servir, maniés avec eirconspection, à établir la véritable parenté de l'ètre qui nous occupe. D’après cela, il me semble que l'Amphioxus vient se placer certainement dans le voisinage cles Ascidies, ainsi que je l'ai représenté dans mon arbre généalogique. De nouvelles eonquêtes dans l'embyologie les animaux conduirent-elles à porter les deux types réunis en une autre place qute celle qui leur revient aujourd'hui, a mes yeux, dans la classification générale? On peut le penser, mais non le prédire avee certitute.

D’ailleurs, si j’ai tenté ici d'exprimer par un arbre généalogrigne élevé suivant la méthode de Darwin les rapports de parenté des ìtres, je n’ai pas la prétention de croire que ma distributiou soit la seule possible; je 11 avais ju'un but : montrer que d'autres hypotheses que celle de Gustra-Thioric sont encore possibles, et qu'une d'entre elles est i fout le moins aussi boune, si mème elle ne concorde pas mienx avec les domnées de l'embryologic que l'hypothèse hackelienne. Eu la presentant, je n'ai pas eru non plus avor' 
tracé le vrai système dı règne animal; je m’en garderais bien, car il est comme lit seience, dont i! est la constaute exjression, variable et perlectible. dinsi mon intention était seulement d'exprimer dans cet essai les rapports les plus généraux des organes nouvellement léconverts des Plagiostomes, de les préciser nettement, afin d'empêeher que quelque autre, laisant une déconverte dans le même sens, n'en reçit senl aussi tout le bénélice.

\title{
RECHERCHES SUR LES PREMIIER PHÉNOMĖNES DU DÉVELOPPEMENT DANS L'OEUF FÉCONDÉ DES NÉMATOIDES ET PULHONES,
}

\author{
Par M. O. Butschli.
}

(Zeitsch. für wiss. Zool., 2 Heft 1875.)

Les phénomènes lont il s'agit sont ceux de la segmentation dans ses rapports avec l'apparition des nouveaux noyaux. M. Bütschli appelait le premier l'attentiou sur ce sujet en 1879, et les résultats publiés deux ans après par Auerbach, ceux que nous communique encore aujourd'hui M. Bütschli, montrent assez qu'il y a la un vaste champ d'exploration jusqu'iei négligé, et d'autant plus étendu même que, l'œuf n’étant pas seulement le germe d'un nouvel organisme, mais eneore l'élément anatomique type en quelque sorte, il y a lieu de se demander jusqu'ì quel point les résultats nouveaux fournis à sou égard ne seraient pas applicables à plusieurs espèces d'éléments anatomiques.

Jusqu'ì ce jour, on n'a soumis aux iuvestigations que l'euf te neuf espèces d'êtres, dont sept sont des Nématoïdes (Ascaris nigrorenosa et Strongylus auricularis, étudiés par Auerbach; Tylenchus nouvean, Cephalobus rigidus, Rhabditis dolichura, Diplogaster et Cucullanus elegans, étudiés par M. Bütschli), el deux, des Gastéroporles (Lymnous uuricularis et Succinaa Pfeifferi). La coneordance des résultats daus ces différents types fait présager, pour les phénomènes que nous allons analyser, une assez grande généralité d'existence, et semble appeler une révision complète le la segmentation et des particularités qui s'y rattachent dans toute l'étendue du règne animal.

Après que dans les Nématö̈les précités la vésicule germinative s’est portée du centre ì la périphérie, et l'expulsion du globule polaire, qui ne serait autre, suivant . II. Bütschli, que la tache germinative elle-nı̂̀me, l'œuf reconstitue un noyau central en lieu et place de la vésicule germinative primitive que l'auteur a vu distinctenent, dans le Cephalobus rigidus, disparaitre complétement en s'étalant et se difusant dans la couche claire de protoplasma qui forme comme un mince manteau au vitellus granuleux. C"est par la récupération d'un noyau que se troltvera constituée ce qu'on appelle la premierc splicie de 
seymentation, celle qui va bientôt se diviser en deux, quatre, ete., sphères secondaires, teruaires, etc. Mais comment un nonveau noyau se produit-il?

La formation part de cette conche de protoplasma dans laq̣uelle la vésicule germinative s'est étalée et qui constitue la zone la plus externe du vitellus. La zone en question augnente graduellement d'épaisseur aux deux pòles de l'œuf et, par une couséfuence forcée, fait hernie d'autant à l’intérieur du vitelius gramuleux: Bientôt, de plus en plus étranglée à la base, cette portion yui a fait hernie se trouve limalement séparée de la zone hyaline, ì laquelle elle se rattichait. Comme eeci s'iccomplit simultanément aux deux pôles, nous arriverons ainsi i la production de deux petits globes de plasma clair et transparent, librement suspendus à l'intérieur du vitellus granuleux. Pour plus de commodité, nommons-les des noyaux partiels.

En règle générale, il s'en produit leux, comme nous venons de le dire; mais, dans le Rhabditis dolichura, M. Bütschli en a vu naitre trois, et jusqu’à eing dans Cucullenus elegars.

Notons également yue les noyaux partiels ne sont pas toujours situés aux deux extrémités du grand axe de l'œuf; quelquefois, au contraire, l'un se formant à l'un des pôles, l'autre se constitue sous la ligune équatoriale; quelquefois encore, les deux preunent naissance au voisiunge immédiat l'un de l'autre.

En quelque nombre et en quelque lieu qu ïls paraissent, une fois produits, les noyaux partiels, en vertu d’une véritable nigration à l'intérieur du vitellus et it la faveur des vifs mouvements amoboïles que ce dernier effectue durant ce temps, marehent ì la rencontre l'un de l'autre; et, que ce soit par un trajet rectiligne et direct, ou au contraire après plus d’un détour, que ce soit aussi dans le centre même de l'cuf ou dans son voisinage, les deux noyaux, venus d'abord en contaet, fusionnent leurs substances de la façon la plus intime et, perdant leur individualité, forment maintenant le noyau unique de la première sphère de segmentation. C'est une véritable copulation, comparable à celle de quelques organismes iuférieurs.

Les résultats précédents concernant exclusivement les Nématoïdes, voyons maintenant pour les Gastéropodes.

Lymnaus d'abord. Laissant de còté les détails intimes relatil's à la disparition de la vésicule germinative et à l'expulsion des globules polaires, l'auteur a ubservé que dans le voisinage du point où ces globules furent produits, se lorment jusqu’à lıuit et même jusqu'à un plus grand nombre de noyaux petits, vésiculiformes, très-transparents, renfermant un certain nombre de granulations foneées, et qu'ici aussi ces noyaux partiels s'unissent successivement, de laçon à ne plus constituer bientòt que deux on trois gros noyaux qui se confonlent définitivement en un seul.

Dans Succinar, an contraire, ne se produisent jamais que deux noyaux parliels, le plus souvent à notable distance l'un de l'autre. Ils se fusionnent d'ailleurs comme dans les cas précédents.

Si nouveaux que les précédents résultats puissent paraitre, la question se laisse poser, question bien digne de l'attention de tous ceux qui se préoccupent de la méthode en zoologie, si, sous l'influence d'une illée théorique, celle yue la division du noyilu précède celle du corps cellulare, les observateurs 
u'anraient pas jusqu'ici interprété constamment le phénomène de la conjugation des noyaux partiels, comme le produit de la division du noyau primaire en deux autres.

Ce que nous venons de voir s'accomplir pour la sphère primaire aurait également lieu pour les sphères de génération ultérieure : elles aussi développeraient a nouveaux frais et par un processus identique, quant au ford, les noyaux que nous leur voyons, noyux qui ne seraient done en aucune sorte une partic aliquote directement transmise par division du noyau de la sphère primitive. C'est au moins ce qu'assure de la facon la plus formelle M. Bütschli pour Cucullanus elegans et Succinca Pfeifferi.

En outre enfin des laits que nous venons de relater, M. Bütschli présente des observations sur un corps jar lui déconvert dans l'œuf du Cucullanus rlegans, corps dont la nature exacte, l'origine, la destinée et la fonction demeurent encore, après les détails que nous livre M. Bütehli, des plus prohléunatiques. A plus forte raison la théorie nouvelle de la fécondation que l'auteur veut baser sur cet élément par lui considéré comme homologue à la capsule spermatique des Infusoires ne peut-elle venir présentement en diseussion. Tous attendrons, pour en rendre compte, que l'écrit que M. Bütschli nous donne à titre de communication provisoire destinée à assurer ses droits à la priorité, soit devenu le mémoire étendu et complet qu'il nous laisse espérer.

\section{A. Sq̧HNEIDER.}

SUR LORGANISATION ET LE DÉVELOPPEMENT DE LAIGUILLON ET DE LOVISCAPTE

DE QUELQUES HYMENOPTÈRES ET DE LA SAUTERELLE VERTE,

Par M. le docteur H. Dewitz.

(Zeilschr. für wiss. Zool, iI heft, 1875).

Les pièces constitutives de l'armature génitale des insectes doivent-elles être morphologiquement considérées comme réductibles ì de simples organes appendiculaires, aux membres spécialement modifiés des derniers zonites de liabdomen ou comme résultant de la transformation particulière des parties axiales mêmes du dernier ou des derniers zonites? La dernière opinion a prévalu jusqüi ce jour et est devenne classique par la belle et minutieuse monugraphie anatonique le l'armature génitale femelle des insectes du professeur de Lacaze-Duthiers. "Lil preuve, dit Gerstacker, dans son manuel si connu de zoologie, tont au long fournie par Lacaze-buthiers, que tous les instruments de ce genre qui, sous lorme de cerceaux, stylets, scies, tenailles on pinces, tarieres, oviscape, aignillon. elc.. sunt silnes it l'extrémité du 
corps avec les configurations les plus diverses ; la preuve, disons-nous, que ces organes ne sont pas des appendices spéciaux, mais doivent ètre envisagés comme des segments ou parties de segments modifiées, est si convaincante, qu'avee elle l'uniformité dans le nombre des segments de l'abbomen, soumis sans cela à de nombreuses variations, so trouve partout rétablie avec une complète concordance."

L’antre opinion, celle qui cousiste au enutraire à voir dans l’armature génitale le produit de la transformation des appenulices des derniers zonites, a été expressement formulée pour la première fois par Packard en 1866. Plus tard, Weismann (1866) et Ganin (1869) se prononcèrent dans le même sens, mais en quelque sorte occasionnellenent et sans faire du point en question l'objet de recherehes étendues et comparatives. Le sujet, toutefois, dont l'inportance pour la morphologie du sqquelette des Articulés est aisée ì concevoir, appela plus particulièrement, en 1872, l'attention de la faculté philosophique de l'Université de Kœenigsbergs, qui le mit all eoneours. Ce fut II. Dewitz qui obtint le prix daus un mẻnoire dont les conclusions sont favorables à l'opinion ‘ui nous oceupe. C'est ee mémoire que public aujourd'hui l'auteur, complété par des reeherches ultérieures. Entre temps, d'ailleurs, Ouljanin (1872) avait publié uı court résumé du développement de l'aignillon de l'Abeille, et Krapelin (1873) un mémoire étendı sur l'aiguillon et la tarière de diffél'ents Hýménoptères.

Les espèces étudiées par 11 . Dewitz sont an nombre de quatre : Locusta viridissima, Apis mellifica, Tespa vulgaris et Cryptus migrator, et pour trois d'entre elles coneernent l'armature génitale mile aussi bien que la femelle.

Nons ne saurions entrer ici lans le létail des observations qui nécessiteraient, pour être facilement comprises, la reproduction de tous les dessins de l'auteur; nens nous bornerons à présenter ses couclusions générales.

Dans toutes les espèces préeitées, le corps eomprend treize segments en arrière de la tête. Dans les Hyménoptères, le dernier, rentrant dans le eorps, réduit à douze le noubre apparent, tandis yu'il le porte il quatorze dans le Locusta, par là division en denx qu’il éprouve durant le développement. L'aignillon de l'Hyménoptère, comme l'oviscapte de la Sauterelle, comprennent six pièces esseutielles dans leur constitution, qui se réduisent à cinq dans les Hyménoptères observés par la coalescence de denx d'entre elles. L'origine de ces six pièces est partout la même : elles waissent de six raeines, dont quatre sont porties par le donzième segment et denx par le onzième.

Ces racines naissent et procèdent des segments de l'fmago de la mème façon Jont Weissmann a représenté la formation des membres du Corethro. L'époque l'apparition des six rateines est dillérente. chloz l’Abeille, la toute jeune larve montre simultanément l'apparition des lenx racines dn onzieme segment et celle des deux racinces intermédiaires du douzième; quant aux deux autres racines de ee segment. les latiriles ou externes, elles ne se dessinent que plus tard. Dans le Locustu, an enntraire, e's deux racines externes du dourième zonite ont déjì alteint daus l'ieuf $11 n$ assez grand degré de développement; les denx racines du onzieme segment y sout accusées sous forme de disques; et quant anx deux racines intermedianes du douzième zonite, elles llapparaissent que quand le jeune insecte a ıéjà quitté l'œuf depuis quıelque 
temps. "C"est-à-dire que nous avons là, dit M. Dewitz, ce qui se voit également ailleurs dans le développement des Articulés, à savoir : que les parties lomologiquement équivalentes ne naissent pas dans un ordre invariabie; ınais que les dimensions et l'importance relatives qu'elles devront aequérir déterminent leur apparition plus précoce ou plus tardive. "Dans les Hyménoptères observés, les deux racines latérales du douzième segment deviennent les étuis ou la gaîne; les deux intermédiaires du uême segment, le gorgeret et les deux racines du onzième zonite, les filets du dard ou les soies de la tarière. Comme les pièces constitutives de l'oviseapte de la Sauterelle naissent de la mème façon, on doit les paralléliser avec leur's corresprondantes chez l'Abeille. Dans ce cas, les valves supérieures du fourreau dans la Sauterelle répondent aux étuis de la gaine de l'Abeille; les valves inférieures de la Sauterelle aux stylets de l'aiguillon, et les valves accessoires de l'oviseapte au gorgeret.

Les zonites ne premnent directement ancune part a la constitution de l'armature.

Quant aux organes màles de la fécondation, ils naissent, chez les Abeilles et lchneumonides d'une part, chez la Sauterelle de l'autre, de deux racines portées par l'avant-dernier segment.

Le développement montre done que les pièces constitutives de l'aiguillon et de l'oviscapte sont des termes répondant aux membres. Reste seulement à savoir si les quatre appendices de l'avant-dernier segment doivent être interprétés comme attestant l'existence de deux paires de membres sur ce zonite ou comme le résultat de la division ou partition d'une seule. La dernière opinion semble la plus probable à N. Dewitz, bien que les faits relatifs au développement des appendices des Arthropodes lui paraissent encore trop peu nombreux pour permettre une solution satisfaisante de la question.

A. S.

Le directeur : H. DE LACAZE-DuthiERS.

Le gérant: $\mathrm{C}$. REINWALD. 




